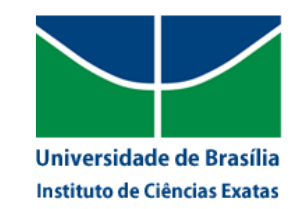

\author{
UNIVERSIDADE DE BRASÍLIA \\ Instituto DE CiênCias Exatas \\ Departamento de Matemática
}

Os polinômios centrais de algumas álgebras associativas Lie nilpotentes

por

Silvio Sandro Alves de Macedo 


\title{
Os polinômios centrais de algumas álgebras associativas Lie nilpotentes
}

\author{
por \\ Silvio Sandro Alves de Macedo
}

Tese apresentada ao Programa de Pós-Graduação do Departamento de Matemática da Universidade de Brasília, como requisito parcial para obtenção do título de Doutor em Matemática.

Área de concentração: Álgebra.

Orientador: Prof. Dr. Alexei Krassilnikov 
Ficha catalográfica elaborada automaticamente, com os dados fornecidos pelo(a) autor(a)

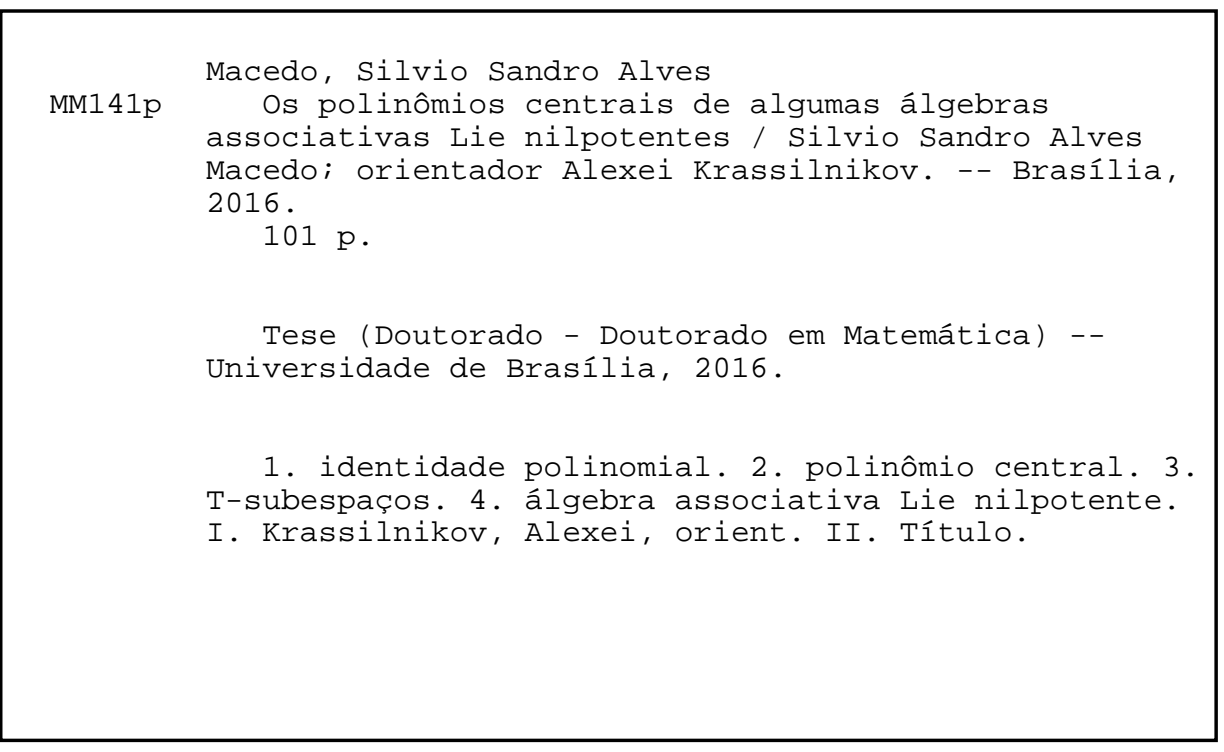


Universidade de Brasília

Instituto de Ciências Exatas

Departamento de Matemática

Os polinômios centrais de algumas álgebras associativas Lie nilpotentes

Por

Silvio Sandro Alves de Macedo

Tese apresentada ao Corpo Docente do Programa de Pós-Graduação em Matemática-UnB, como requisito parcial para obtenção do grau de

DOUTOR EM MATEMÁTICA

Brasília, 26 de agosto de 2016.

Comissão Examinadora:

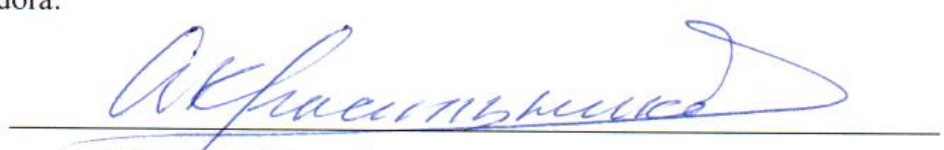

Prof. Dr. Alexei Krassilnikov - Orientador (MAT-UnB)

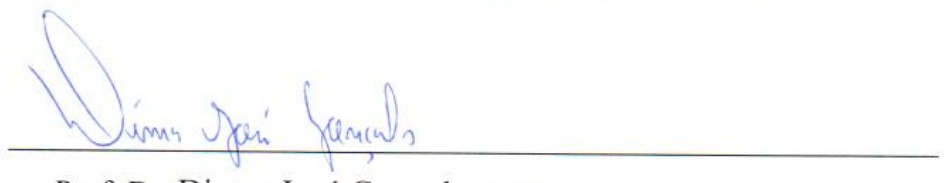

Prof. Dr. Dimas José Gonçalves (UFScar)

plusblor var

Prof. Dr. Plamen Emilov Kochloukov (UNICAMP)

Almigonus S Pmils

Profa. Dra. Aline Gomes da Silva Pinto (UnB)

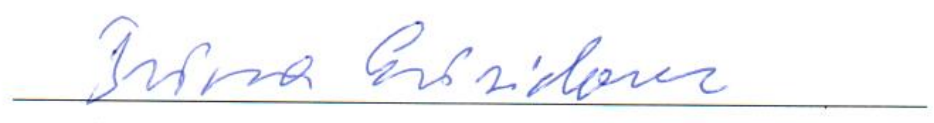

Profa. Dra. Irina Sviridova (UnB) 
Todos os direitos reservados. É proibida a reprodução total ou parcial do trabalho sem autorização da universidade, do autor e do orientador. 
Este trabalho é dedicado a você mesmo, caro leitor. 


\section{Agradecimentos}

Como a lista de pessoas que me ajudaram é longa e o espaço não tão longo assim, vou citar apenas aquelas que estiveram mais próximas nesses últimos anos. Eudes, Raimundo e Jorge, obrigado pelo incentivo quando eu ainda era um aluno especial. O curso de Análise Funcional do Prof. Carlos Alberto ficará guardado na minha memória. Obrigado também aos ex-alunos de doutorado Alex (Paraíba), José Carlos (Zé), Edimilson, Keidna, Ilana, Claud e Bruno Trindade por ajudarem na preparação do meu exame de qualificação.

Gostaria de agradecer ao meu orientador, $\operatorname{Prof}^{\mathrm{o}}$ Alexei, por sua experiência, ensinamentos e paciência infinita.

Gostaria de agradecer à Banca Examinadora: Prof. Dimas, Prof. Plamen, Profa. Aline, Profa. Irina e Prof. Victor. As correções apontadas por vocês foram muito valiosas. Tive o prazer de ter sido aluno do Prof. Dimas. Sua didática para ensinar ideias difíceis é tão grande que diminui o atrito a quase zero.

Vieram lá do Mato Grosso para assistir a minha defesa a minha mãe Madalena, o meu pai Bonifácio, a minha madrinha Lenira e o meu tio Daniel. O momento mais feliz da minha infância foi na casa da minha madrinha, fazendo bagunça. O meu tio eu chamava de Danielzão porque antes ele parecia grande. O seu coração continua enorme.

Obrigado ao meu amigo Adriano Cavalcante Bezerra, pela amizade, pelos conselhos, pelas caronas e pelas piadas quase todas com graça.

Obrigado ao meu amigo Agenor Freitas de Andrade. Conheci ele no ano de 2004 no curso de Graduação em Matemática na UFMT, fizemos o mestrado na UFG e agora terminamos o doutorado quase juntos aqui na Unb. Na metade da Graduação lembro de você entrando na sala e dizendo - Cara, vou ser pai! Era a Ana Júlia, depois veio a Carol e o Eduardo. Você se saiu muito bem. A Jeisa (Psicóloga) veio assitir a minha defesa. Isso que é amizade.

Por fim, gostaria de agradecer à equipe maravilhosa do IFG Campus Luziânia. Estive afastado por três anos das minhas atividades para dedicar ao doutorado. Agradeço aos gestores desse período pelo apoio total e irrestrito: Prof. José Carlos, Profa. Oneida e Profa. Marizângela (Mary). 
O Coelho Branco pôs os óculos.

- Por onde devo começar, Vossa Magestade? — perguntou ele.

- Começa pelo princípio, - disse o Rei, muito sério - e continua até chegares ao fim. Depois para.

Lewis Carroll, As Aventuras de Alice no País das Maravilhas. 


\section{Resumo}

Nesta tese estudamos os polinômios centrais de algumas álgebras associativas Lie nilpotentes universais. Elas são definidas por $Q_{n}=F\langle X\rangle / T^{(n)}$ (e também são conhecidas como álgebras associativas Lie nilpotentes relativamente livres) onde $F$ é um corpo, $F\langle X\rangle$ é a álgebra associativa livre unitária, livremente gerada pelo conjunto enumerável $X=\left\{x_{0}, x_{1}, x_{2}, \ldots\right\}$ e $T^{(n)}$ é o ideal bilateral de $F\langle X\rangle$ gerado pelos comutadores $\left[a_{1}, \ldots, a_{n}\right], a_{i} \in F\langle X\rangle$. O nosso primeiro resultado principal é uma descrição dos polinômios centrais da álgebra $Q_{4}$ quando $\operatorname{char}(F)=3$. Nosso segundo resultado principal é uma descrição dos polinômios centrais da álgebra $Q_{4}$ quando $\operatorname{char}(F)=2$.

Os polinômios centrais da $F$-álgebra $Q_{4}$ quando $\operatorname{char}(F) \neq 2,3$ foram descritos por Grishin (2012). Se $\operatorname{char}(F) \neq 3$, então $\left[x_{1}, x_{2}\right]\left[x_{3}, x_{4}, x_{5}\right]$ pertence a $T^{(4)}$ (Volichenko, 1978). Isso implica que a imagem de $T^{(3)}$ em $Q_{4}$ é central nessa álgebra, o que permite reduzir o problema da descrição dos polinômios centrais da álgebra $Q_{4}$ para um problema sobre elementos da álgebra $Q_{3}$. Porém, se $\operatorname{char}(F)=3$,

então $\left[x_{1}, x_{2}\right]\left[x_{3}, x_{4}, x_{5}\right]$ não pertence a $T^{(4)}$ (Krasilnikov, 2013). Por essa razão, a descrição dos polinômios centrais da $F$-álgebra $Q_{4}$ quando $\operatorname{char}(F)=3$ é mais sofisticada do que quando $\operatorname{char}(F) \neq 3$. Se $\operatorname{char}(F)=2$, então $x_{0}^{2}+T^{(4)}$ não é central em $Q_{4}$. Isso implica que a descrição dos polinômios centrais de $Q_{4}$ é ligeiramente diferente do caso de $\operatorname{char}(F) \neq 2,3$.

O nosso terceiro resultado principal é uma descrição dos geradores da álgebra $Q_{4}$ como espaço vetorial quando $\operatorname{char}(F)>3$. Esse resultado é uma generalização do resultado de Grishin. Também obtivemos uma descrição dos polinômios hipercentrais das álgebras $Q_{4}$ e $Q_{5}$. Um polinômio hipercentral é uma generalização de polinômio central. Essa generalização foi introduzida por Laue (1984).

\section{Palavras-chave}

identidade polinomial; polinômio central; $T$-subespaço; álgebra associativa Lie nilpotente 


\section{Abstract}

In this $\mathrm{PhD}$ thesis we study the central polinomials of some universal Lie nilpotent associative algebras. They are defined by $Q_{n}=F\langle X\rangle / T^{(n)}$ (and also are called relatively free Lie nilpotent associative algebras) where $F$ is a field, $F\langle X\rangle$ is the free unital associative algebra freely generated by the infinite countable set $X=\left\{x_{0}, x_{1}, x_{2}, \ldots\right\}$ and $T^{(n)}$ is the two-sided ideal of $F\langle X\rangle$ generated by the commutators $\left[a_{1}, \ldots, a_{n}\right], a_{i} \in F\langle X\rangle$. Our first main result is a description of the central polynomials of the algebra $Q_{4}$ when $\operatorname{char}(F)=3$. Our second main result is a description of the central polynomials of the algebra $Q_{4}$ when $\operatorname{char}(F)=2$.

The central polynomials of the $F$-algebra $Q_{4}$ when $\operatorname{char}(F) \neq 2,3$ have been described by Grishin (2012). If $\operatorname{char}(F) \neq 3$, then $\left[x_{1}, x_{2}\right]\left[x_{3}, x_{4}, x_{5}\right]$ belongs to $T^{(4)}$ (Volichenko, 1978). This implies that the image of $T^{(3)}$ in $Q_{4}$ is central in this algebra that allows us to reduce the problem of description of the central polynomials of the algebra $Q_{4}$ to a problem about elements of the algebra $Q_{3}$. However, if $\operatorname{char}(F)=3$, then $\left[x_{1}, x_{2}\right]\left[x_{3}, x_{4}, x_{5}\right]$ does not belong to $T^{(4)}$ (Krasilnikov, 2013). For this reason the description of the central polynomials of the $F$-algebra $Q_{4}$ when $\operatorname{char}(F)=3$ is more sophisticated than in the case when $\operatorname{char}(F) \neq 3$. If $\operatorname{char}(F)=2$, then $x_{0}^{2}+T^{(4)}$ is not central in $Q_{4}$. This implies that the description of the central polynomials of $Q_{4}$ is slightly different from the case $\operatorname{char}(F) \neq 2,3$.

Our third main result is a description of generators of the algebra $Q_{4}$ as a vector space when $\operatorname{char}(F)>3$. This result is a generalization of result of Grishin's result. We also obtain a description of the hipercentral polynomials of the algebras $Q_{4}$ and $Q_{5}$. A hipercentral polynomial is a generalization of a central polynomial. This generalization was introduced by Laue (1984).

\section{Keywords}

polynomial identity; central polynomial; $T$-subspace; Lie nilpotent associative algebra 


\section{Sumário}

Introdução

1 Preliminares $\quad 18$

$\begin{array}{ll}1.1 & \text { Identidades polinomiais e } T \text {-ideais } \\ & 18\end{array}$

1.2 Polinômios centrais e $T$-subespaços 25

1.3 Relações na álgebra $Q_{3} \quad 26$

1.4 Polinômios centrais da álgebra $Q_{3} \quad 31$

1.5 Relações na álgebra $Q_{4} \quad 37$

2 Polinômios centrais da álgebra $Q_{4} \quad 42$

2.10 caso de característica $\neq 3 \quad 42$

2.2 O caso de característica $3 \quad 53$

2.2.1 Uma base para o espaço vetorial $Q_{4} \quad 53$

2.2.2 Uma base para o espaço vetorial $T^{(3)} / T^{(4)} \quad 58$

2.2.3 Os polinômios centrais da álgebra $C\left(Q_{4}\right) \cap T^{(3)} \quad 64$

2.2.4 Os geradores do espaço vetorial $\left(C\left(Q_{4}\right)+T^{(3)}\right) / T^{(3)} \quad 76$

2.2.5 Os polinômios centrais da álgebra $Q_{4} \quad 81$

3 Polinômios hipercentrais $\quad 84$

3.1 A álgebra $Q_{4} \quad 85$

3.2 A álgebra $Q_{5} \quad 90$

3.3 Resumo dos resultados 96

$\begin{array}{ll}\text { Referências Bibliográficas } & 97\end{array}$

$\begin{array}{ll}\text { Índice Remissivo } & 101\end{array}$ 


\section{Introdução}

A área da matemática na qual esta tese está situada é álgebra. Mais precisamente, fazemos uma contribuição para a teoria que estuda álgebras que satisfazem alguma identidade polinomial. Tais álgebras são chamadas de PI-álgebras (do inglês Polynomial Identity) e formam uma classe abrangente de álgebras, que incluem algumas álgebras muito importantes para outras áreas da ciência, como as álgebras de matrizes e as álgebras de Grassmann (ou álgebras exteriores).

À menos que se diga o contrário, as álgebras consideradas neste trabalho serão sempre associativas e unitárias. As definições formais dos conceitos apresentados nesta introdução encontram-se no Capítulo 1.

Seja $A$ uma álgebra sobre um corpo $F$. Um polinômio $f\left(x_{1}, \ldots, x_{n}\right)$ nas variáveis não comutativas $x_{1}, \ldots, x_{n}$ com coeficientes em $F$ é uma identidade polinomial para a álgebra $A$ se $f\left(a_{1}, \ldots, a_{n}\right)=0$ para quaisquer elementos $a_{1}, \ldots, a_{n} \in A$.

Apesar da ideia de identidade polinomial aparecer implicitamente em trabalhos anteriores, o interesse geral em PI-álgebras começou após a publicação de um artigo de Kaplansky [30] em 1948; esse trabalho está inserido no que hoje é chamada de teoria estrutural de PI-álgebras, que visa obter informações sobre a estrutura de uma álgebra quando se sabe que ela satisfaz alguma identidade polinomial.

Em 1950, Amitsur e Levitzki [1] provaram por métodos puramente combinatórios que o polinômio padrão de grau $2 n$

$$
s_{2 n}\left(x_{1}, \ldots, x_{2 n}\right)=\sum_{\sigma \in S_{2 n}}(-1)^{\sigma} x_{\sigma(1)} \ldots x_{\sigma(2 n)}
$$

é uma identidade polinomial de grau minimal para a álgebra de matrizes $n \times n$ com entradas em um corpo qualquer. Esse trabalho inaugurou uma nova abordagem às PI-álgebras, sendo o principal objetivo a descrição das identidades polinomiais de uma dada álgebra.

Seja $F\langle X\rangle$ a álgebra dos polinômios nas variáveis não comutativas $X=$ $\left\{x_{0}, x_{1}, x_{2}, \ldots\right\}$ com coeficientes no corpo $F$. O conjunto $I d(A)$ de todas as identidades polinomiais de uma álgebra $A$ forma um ideal bilateral de $F\langle X\rangle$. Mais ainda, $\operatorname{Id}(A)$ é fechado por todos os endomorfismos de $F\langle X\rangle$. Ideais de $F\langle X\rangle$ com essa propriedade 
são chamados de $T$-ideais. Observe que o estudo de $T$-ideais se reduz ao estudo de identidades polinomiais. Mais precisamente, se $I$ é um $T$-ideal de $F\langle X\rangle$ e $A=F\langle X\rangle / I$, então $I=I d(A)$.

Um $T$-ideal $I$ é gerado por um conjunto $S \subseteq F\langle X\rangle$ se $I$ for o menor $T$-ideal de $F\langle X\rangle$ que contém $S$. Um $T$-ideal é finitamente gerado quando é gerado por um conjunto finito.

Antes de continuar, vamos definir uma álgebra que desempenha um papel muito importante em PI-álgebras.

Sejam $F$ um corpo infinito de característica $\neq 2$ e $V$ um espaço vetorial sobre $F$ com base enumerável $e_{1}, e_{2}, \ldots$ A álgebra de Grassmann infinitamente gerada e unitária de $V$, denotada por $E$, é a álgebra associativa gerada por $e_{1}, e_{2}, \ldots$ e com relações $e_{i} e_{j}=-e_{j} e_{i}$ para quaisquer $i$ e $j$.

São poucas as álgebras para as quais se conhece uma descrição completa das suas identidades polinomiais. Uma lista quase completa delas é

$$
E, E \otimes E, M_{2}(F) \text { e } U_{n}(F)
$$

onde $E \otimes E$ é o produto tensorial da álgebra de Grassmann $E ; M_{2}(F)$ e $U_{n}(F)$ são respectivamente a álgebra de matrizes $2 \times 2$ e a álgebra de matrizes triangulares superiores $n \times n$, ambas com entradas no corpo $F$. Mesmo nessa lista, as identidades de algumas álgebras não são conhecidas para todos os corpos nos quais elas estão definidas. Para mais detalhes consulte [13].

Uma questão se coloca: podem as identidades polinomiais de uma álgebra serem descritas "de maneira finita"? Mais precisamente: é todo $T$-ideal de $F\langle X\rangle$ finitamente gerado como um $T$-ideal? Quando a característica do corpo $F$ é 0 , esse é o famoso Problema de Specht, proposto por Specht [42] em 1950.

Diversos casos particulares do Problema de Specht foram resolvidos nos anos seguintes mas uma prova completa (afirmativa) só foi dada em 1987 por Kemer, após uma série de artigos (veja [31]). Entretanto, sobre um corpo $F$ de característica $p>0$, existem $T$-ideais de $F\langle X\rangle$ que não são finitamente gerados. Isso foi provado em 1999 por Belov [6], Grishin [24] e Shchigolev [40]. A construção desses $T$-ideais faz uso de $T$-subespaços de $F\langle X\rangle$ não finitamente gerados, construídos por Grishin [24] para $p=2$ e por Shchigolev [41] para $p>2$.

Seja $Z(A)$ o centro de uma álgebra $A$. Dizemos que $f\left(x_{1}, \ldots, x_{n}\right) \in F\langle X\rangle$ é um polinômio central de $A$ se $f\left(a_{1}, \ldots, a_{n}\right) \in Z(A)$ para quaisquer $a_{1}, \ldots, a_{n} \in A$.

O conjunto $C(A)$ de todos os polinômios centrais de $A$ forma um subespaço vetorial de $F\langle X\rangle$. Mais ainda $C(A)$ é fechado por todos os endomorfismos de $F\langle X\rangle$. Subespaços vetoriais de $F\langle X\rangle$ com essa propriedade são chamados de $T$-subespaços. 
Ao contrário de $T$-ideais, o estudo dos $T$-subespaços não se reduz ao estudo dos polinômios centrais. Mais precisamente, existem $T$-subespaços $I$ de $F\langle X\rangle$ para os quais não existe uma álgebra $A$ tal que $I=C(A)$ (veja [20, Observação 1]).

Um $T$-subespaço $I$ é gerado por um conjunto $S \subseteq F\langle X\rangle$ se $I$ for o menor $T$-subespaço de $F\langle X\rangle$ que contém $S$. Um $T$-subespaço é finitamente gerado quando é gerado por um conjunto finito.

Resultados semelhantes àqueles de $T$-ideais foram obtidos para $T$ subespaços. Se $F$ é um corpo de característica 0 , então todo $T$-subespaço de $F\langle X\rangle$ é finitamente gerado como um $T$-subespaço; esse resultado foi provado por Shchigolev [39], em 2001.

Entretanto, sobre um corpo $F$ de característica $p>0$, existem $T$-subespaços de $F\langle X\rangle$ que não são finitamente gerados como $T$-subespaços. Além dos exemplos citados acima, foi mostrado recentemente (veja $[4,7]$ ) que o $T$-subespaço dos polinômios centrais da álgebra de Grassmann (sobre um corpo de característica $p>2$ ) não é finitamente gerado como um $T$-subespaço (detalhes adiante).

Seja $f$ um polinômio central de uma álgebra $A$. Se $f$ não é uma identidade polinomial de $A$ e também não possui termos escalares, dizemos que $f$ é um polinômio central próprio de $A$. Na literatura, com frequência, "polinômio central" significa "polinômio central próprio"(veja [13]).

O interesse por polinômios centrais começou em 1956, quando Kaplansky [29] perguntou se a álgebra de matrizes $M_{n}(F), \operatorname{com} n>2$, possuía algum polinômio central próprio. A resposta afirmativa para o problema proposto por Kaplansky foi dada independentemente por Formanek [17], em 1972 e por Razmyslov [38], em 1973. Eles apresentaram dois métodos diferentes para a construção de polinômios centrais da álgebra $M_{n}(F)$ (veja [13] para uma exposição dos dois métodos).

A partir daí, vários outros polinômios centrais para a álgebra de matrizes foram obtidos. Entretanto, uma descrição completa dos polinômios centrais de $M_{n}(F)$ é conhecida apenas quando $n=2$ e o corpo $F$ é infinito de característica $\neq 2$. Quando a característica de $F$ é 0 , os geradores de $C\left(M_{2}(F)\right)$ como um $T$-subespaço foram obtidos por Okhitin [37], em 1988 (veja também [15]). Quando $F$ é infinito de característica $\neq 2$, os geradores de $C\left(M_{2}(F)\right)$ como um $T$-subespaço foram obtidos por Colombo e Koshlukov [8], em 2004.

São muito poucas as álgebras para as quais se tem uma descrição completa dos seus polinômios centrais. A álgebra de matrizes $M_{2}(F)$, a álgebra de Grassmann $E$ e a álgebra $Q_{4}=F\langle X\rangle / T^{(4)}$ (abordada nesta tese) são quase todos os exemplos conhecidos até este momento.

As identidades polinomiais da álgebra de Grassmann sobre um corpo de característica 0 foram descritas primeiramente por Krakowski e Regev [32], em 1973 
(veja também Latyshev [35]). Essas identidades coincidem com as identidades da Álgebra de Gassmann sobre um corpo de característica positiva (veja [18]). Assim, foi mostrado por esses autores que se $F$ é um corpo infinito de característica $\neq 2$, então $I d(E)$ é gerado como $T$-ideal pelo polinômio $\left[x_{1}, x_{2}, x_{3}\right]=\left[\left[x_{1}, x_{2}\right], x_{3}\right]$, onde $\left[x_{1}, x_{2}\right]=$ $x_{1} x_{2}-x_{2} x_{1}$.

A descrição dos polinômios centrais da álgebra de Grassmann $E$ foi obtida em 2010, independentemente por Bekh-Ochir e Rankin [3], por Brandão Jr, Koshlukov, Krasilnikov e Silva [7] e por Grishim [23]. Seguindo a notação de [7], seja $q\left(x_{1}, x_{2}\right)=x_{1}^{p-1}\left[x_{1}, x_{2}\right] x_{2}^{p-1}$, e para cada $n \geq 1$, defina

$$
q_{n}=q_{n}\left(x_{1}, \ldots, x_{2 n}\right)=q\left(x_{1}, x_{2}\right) q\left(x_{3}, x_{4}\right) \ldots q\left(x_{2 n-1}, x_{2 n}\right)
$$

Teorema 0.1 ([3, 7, 23]). Seja $F$ um corpo infinito de característica $p>2$. Então $C(E)$, o espaço vetorial dos polinômios centrais da álgebra de Grassmann E, é gerado (como $T$-subespaço de $F\langle X\rangle$ ) pelos polinômios

$$
x_{1}\left[x_{2}, x_{3}, x_{4}\right], x_{0}^{p}, x_{0}^{p} q_{1}, x_{0}^{p} q_{2}, \ldots, x_{0}^{p} q_{n}, \ldots
$$

Mais ainda, foi provado em [4] e [7] que $C(E)$ não é finitamente gerado como $T$-subespaço. Esse foi o primeiro exemplo de uma álgebra onde o seu $T$-subespaço de polinômios centrais não é finitamente gerado.

Se $F$ possui característica 0 , então $C(E)$ é gerado como $T$-subespaço por 1 e pelos polinômios $x_{1}\left[x_{2}, x_{3}, x_{4}\right]$ e $\left[x_{1}, x_{2}\right]$ (veja [3] e [7]).

Sejam $a_{1}, \ldots, a_{n}$ elementos de uma álgebra $A$. O comutador de comprimento 2 é definido por $\left[a_{1}, a_{2}\right]=a_{1} a_{2}-a_{2} a_{1}$. O comutador de comprimento $n \geq 3$ é definido recursivamente por

$$
\left[a_{1}, \ldots, a_{n-1}, a_{n}\right]=\left[\left[a_{1}, \ldots, a_{n-1}\right], a_{n}\right] .
$$

Seja $T^{(n)}$ o ideal bilateral de $F\langle X\rangle$ gerado por todos os comutadores $\left[a_{1}, a_{2}, \ldots, a_{n}\right]$, onde $a_{i} \in F\langle X\rangle$. A álgebra $Q_{n}=F\langle X\rangle / T^{(n)}$ é chamada álgebra associativa Lie nilpotente universal de classe $n-1$ ou ainda álgebra relativamente livre na classe de álgebras definidas pela identidade $\left[x_{1}, x_{2}, \ldots, x_{n}\right]=0$.

O grau de um monômio $u=x_{i_{1}} \ldots x_{i_{n}}$ em $x_{i}$ é o número de ocorrências de $x_{i}$ em $u$. Um polinômio $f=f\left(x_{1}, \ldots, x_{n}\right)$ é multi-homogêneo de multi-grau $\left(m_{1}, \ldots, m_{n}\right)$ se todo monômio de $f$ possui grau $m_{i}$ em $x_{i}$ para cada $i=1, \ldots, n$.

Seja $f\left(x_{1}, \ldots, x_{n}\right) \in F\langle X\rangle$ um polinômio qualquer, podemos sempre escrever

$$
f=\sum_{m_{1} \geq 0, \ldots, m_{n} \geq 0} f^{\left(m_{1}, \ldots, m_{n}\right)}
$$


onde $f^{\left(m_{1}, \ldots, m_{n}\right)}$ é a soma de todos os monômios de $f$ com multi-grau $\left(m_{1}, \ldots, m_{n}\right)$. Os polinômios $f^{\left(m_{1}, \ldots, m_{n}\right)}$ são chamados de componentes multi-homogêneas de $f$.

Seja $I$ um $T$-ideal de $F\langle X\rangle$ e $f \in I$ um polinômio qualquer. Se todas as componentes multi-homogêneas de $f$ ainda pertencerem a $I$, dizemos que $I$ é um $T$-ideal multi-homogêneo. De modo análogo se define $T$-subespaço multi-homogêneo.

Quando $F$ é um corpo infinito, todos os $T$-ideais e $T$-subespaços de $F\langle X\rangle$ são multi-homogêneos.

Entretanto o $T$-subespaço dos polinômios centrais da álgebra $Q_{n}=$ $F\langle X\rangle / T^{(n)}$ é muti-homogêneo para qualquer corpo $F$. Isso é consequência do fato de $T^{(n)}$ ser um $T$-ideal multi-homogêneo de $F\langle X\rangle$ para qualquer corpo $F$.

Recentemente, têm sido objeto de interesse a descrição dos polinômios centrais da álgebra $Q_{n}$ (veja [22] e [25]).

O espaço vetorial $C\left(Q_{3}\right)$ dos polinômios centrais da álgebra $Q_{3}=F\langle X\rangle / T^{(3)}$ é gerado como $T$-subespaço pelos polinômios (0-1) quando $F$ é um corpo qualquer de característica $p$ e por 1 e pelos polinômios $\left[x_{1}, x_{2}\right]$ e $x_{1}\left[x_{2}, x_{3}\right]$ quando $F$ possui característica 0 . Isso pode ser deduzido dos resultados de [7] e está feito em detalhes no Capítulo 1.

A descrição dos polinômios centrais da álgebra $Q_{4}=F\langle X\rangle / T^{(4)}$ quando $F$ possui característica $>3$ foi obtida por Grishin [22], em 2012. Usando a mesma notação de [7], o resultado de Grishin pode ser escrito da seguinte forma:

Teorema 0.2 ([22], Teorema 2). Seja $F$ um corpo infinito de característica $p>3$. Então $C\left(Q_{4}\right)$, o espaço vetorial dos polinômios centrais da álgebra $Q_{4}=F\langle X\rangle / T^{(4)}$, é gerado como $T$-subespaço de $F\langle X\rangle$ pelos polinômios

$$
x_{1}\left[x_{2}, x_{3}, x_{4}\right], x_{0}^{p}, x_{0}^{p} q_{2}, \ldots, x_{0}^{p} q_{n}, \ldots
$$

Na verdade, como foi observado por A. Krasilnikov em comunicação particular, a demonstração do Teorema 0.2 dada por Grishin [22] estava incompleta. No entanto, como veremos adiante, o Teorema 0.2 é um corolário de um dos resultados principais desta tese.

Quando $F$ possui característica $0, C\left(Q_{4}\right)$ é gerado como $T$-subespaço por 1 e pelos polinômios $\left[x_{1}, x_{2}\right]\left[x_{3}, x_{4}\right]$ e $x_{1}\left[x_{2}, x_{3}, x_{4}\right]$ (veja [25]).

Se $F$ possui característica $\neq 3$, então $\left[x_{1}, x_{2}\right]\left[x_{3}, x_{4}, x_{5}\right]$ pertence a $T^{(4)}$ (veja [44]). Isso implica que a imagem de $T^{(3)}$ em $Q_{4}$ é central nessa álgebra, o que permite reduzir o problema da descrição dos polinômios centrais da álgebra $Q_{4}$ a um problema sobre os elementos da álgebra $Q_{3}$. No entanto, se $F$ possui característica 3 , então $\left[x_{1}, x_{2}\right]\left[x_{3}, x_{4}, x_{5}\right]$ não pertence a $T^{(4)}$ (veja [33]). Por esse motivo, a descrição dos 
polinômios centrais da álgebra $Q_{4}$ para $F$ de característica 3 é muito mais sofisticada do que no caso no qual $F$ possui característica $\neq 3$.

Lembrando que se $F$ possui característica 3, então

$$
q_{n}=q_{n}\left(x_{1}, \ldots, x_{2 n}\right)=x_{1}^{2}\left[x_{1}, x_{2}\right] x_{2}^{2} \ldots x_{2 n-1}^{2}\left[x_{2 n-1}, x_{2 n}\right] x_{2 n}^{2},
$$

vamos definir $u_{0}=u_{0}\left(x_{1}, x_{2}, x_{3}\right)=x_{1}^{2} x_{2}^{2} x_{3}^{2}\left[x_{1}, x_{2}, x_{3}\right]$, e para cada $n \geq 1$,

$$
u_{n}=u_{n}\left(x_{1}, \ldots, x_{2 n+3}\right)=q_{n}\left(x_{1}, x_{2}, \ldots, x_{2 n}\right) u_{0}\left(x_{2 n+1}, x_{2 n+2}, x_{2 n+3}\right) .
$$

Defina também $\omega\left(x_{1}, x_{2}, x_{3}, x_{4}\right)=\left[x_{1}, x_{2}\right]\left[x_{3}, x_{4}\right]+\left[x_{1}, x_{3}\right]\left[x_{2}, x_{4}\right]$. O nosso primeiro resultado principal é o seguinte:

Teorema 2.10 Seja $F$ um corpo de característica 3. Então $C\left(Q_{4}\right)$, o espaço vetorial dos polinômios centrais da álgebra $Q_{4}=F\langle X\rangle / T^{(4)}$ é gerado, como $T$-subespaço de $F\langle X\rangle$, pelos polinômios

(i) $x_{1} \omega\left(x_{2}, x_{3}, x_{4}, x_{5}\right),\left[x_{1}, x_{2}\right]\left[x_{3}, x_{4}\right]-x_{1}\left[x_{2}, x_{3}, x_{4}\right], x_{1}\left[x_{2}, x_{3}, x_{4}, x_{5}\right]$,

(ii) $x_{0}^{3}, x_{0}^{3} q_{3}, x_{0}^{3} q_{6}, \ldots, x_{0}^{3} q_{3 n}, \ldots$,

(iii) $x_{0}^{3} u_{0}, x_{0}^{3} u_{1}, x_{0}^{3} u_{2}, \ldots, x_{0}^{3} u_{n}, \ldots$

Como uma consequência do Teorema 2.10 e do Teorema 3 em [7], obtemos o seguinte:

Corolário 2.32 Seja F um corpo de característica 3. Então o espaço vetorial $C\left(Q_{4}\right)$ dos polinômios centrais da álgebra $Q_{4}=F\langle X\rangle / T^{(4)}$ não é finitamente gerado como $T$-subespaço de $F\langle X\rangle$.

Se $F$ possui característica 2, então $x_{0}^{2}+T^{(4)}$ não é central em $Q_{4}$. Isso implica que a descrição dos polinômios centrais de $Q_{4}$ é ligeiramente diferente do caso no qual $F$ possui característica $>3$. O nosso segundo resultado principal é uma descrição dos polinômios centrais da álgebra $Q_{4}$ quando $F$ possui característica 2 .

Teorema 2.1 Seja $F$ um corpo de característica 2. Então $C\left(Q_{4}\right)$, o espaço vetorial dos polinômios centrais da álgebra $Q_{4}=F\langle X\rangle / T^{(4)}$, é gerado como $T$-subespaço de $F\langle X\rangle$ pelos polinômios

$$
x_{1}\left[x_{2}, x_{3}, x_{4}\right], x_{0}^{4}, x_{0}^{2} q_{2}, x_{0}^{2} q_{3}, x_{0}^{2} q_{4}, \ldots, x_{0}^{2} q_{n}, \ldots
$$

Seja $M$ o conjunto dos monômios mônicos de $F\langle X\rangle$, isto é

$$
M=\left\{x_{i_{1}} x_{i_{2}} \ldots x_{i_{n}}: x_{i_{s}} \in X\right\}
$$


O nosso terceiro resultado principal é uma generalização do resultado de Grishin [22](Teorema 0.2 acima). Ele fornece uma descrição mais precisa dos polinômios centrais da álgebra $Q_{4}$ quando $F$ é um corpo de característica $>3$.

Teorema 2.2 Seja $F$ um corpo de característica $p>3$. Então $C\left(Q_{4}\right)$, o espaço vetorial dos polinômios centrais da álgebra $Q_{4}=F\langle X\rangle / T^{(4)}$ é gerado (como espaço vetorial) pelos polinômios

(i) $\left[a_{1}, a_{2}\right]\left[a_{3}, a_{4}\right], a_{1}\left[a_{2}, a_{3}, a_{4}\right], a_{i} \in M$,

(ii) $x_{i_{1}}^{p r_{1}} \ldots x_{i_{k}}^{p r_{k}} x_{j_{1}}^{p-1}\left[x_{j_{1}}, x_{j_{2}}\right] x_{j_{2}}^{p-1} \ldots x_{j_{2 l-1}}^{p-1}\left[x_{j_{2 l-1}}, x_{j_{2 l}}\right] x_{j_{2 l}}^{p-1}$, onde $k \geq 0, l \geq 2, r_{s}>0, i_{1}<$ $\ldots<i_{k}, j_{1}<\ldots<j_{2 l}$.

Sejam $f=f\left(x_{1}, \ldots, x_{n}\right) \in F\langle X\rangle$ um polinômio e $y_{1}, \ldots, y_{m} \in X$ variáveis tais que $\left\{x_{1}, \ldots, x_{n}\right\} \cap\left\{y_{1}, \ldots, y_{m}\right\}=\varnothing$. Dizemos que $f$ é um polinômio $m$-central de uma álgebra $A$ se $\left[f, y_{1}, \ldots, y_{m}\right]$ pertence a $\operatorname{Id}(A)$. Os polinômios $m$-centrais de $A, m \geq 2$ são chamados genericamente de polinômios hipercentrais de $A$. Observe que um polinômio 1-central é polinômio central. Assim os polinômios hipercentrais são uma generalização dos polinômios centrais. Essa genealização foi introduzida (com outra terminologia) por Laue [36] no contexto de anéis associativos.

O nosso último resultado principal é um conjunto de várias proposições que fornecem uma descrição dos polinômios hipercentrais das álgebras $Q_{4}$ e $Q_{5}$.

Esta tese está organizada da seguinte forma: no Capítulo 1 estão as definições e resultados bem conhecidos que foram usados no texto. O Capítulo 2 é o mais importante desta tese, nele estão demonstrados os nossos três resultados principais, a saber o Teorema 2.1, o Teorema 2.2 e o Teorema 2.10. No Capítulo 3 damos uma descrição completa dos polinômios hipercentrais das álgebras $Q_{4}$ e $Q_{5}$. 


\section{Preliminares}

Este capítulo faz uma síntese dos resultados conhecidos que serão usados no decorrer desta tese. Ele foi escrito com base nos livros [13, 19] e demais artigos citados ao longo do texto.

\subsection{Identidades polinomiais e $T$-ideais}

O conjunto dos números naturais será denotado por $\mathbb{N}=\{1,2,3, \ldots\}$.

Lembremos que uma álgebra $A$ sobre um corpo $F$ é um $F$-espaço vetorial munido de uma multiplicação $A \times A \longrightarrow A,(a, b) \rightarrow a b$ que satisfaz, para quaisquer $a, b, c \in A$ e $\lambda \in F$, as seguintes propriedades:

$$
\begin{aligned}
& a(b+c)=a b+a c, \\
& (a+b) c=a c+b c, \\
& \lambda(a b)=(\lambda a) b=a(\lambda b) .
\end{aligned}
$$

Seja $A$ uma álgebra sobre um corpo $F$. Dizemos que

$A$ é associativa se $(a b) c=a(b c)$, para quaisquer $a, b, c \in A$,

$A$ é comutativa se $a b=b a$, para quaisquer $a, b \in A$,

$A$ é unitária se existir um elemento $1 \in A$ tal que $1 a=a=a 1$ para todo $a \in A$.

Seja $I$ um subespaço vetorial de uma álgebra $A$. Se $a b \in I$ sempre que $a, b \in I$, dizemos que $I$ é uma subálgebra de $A$. Se $A$ for unitária com unidade 1, então para ser subálgebra $I$ deve satisfazer ainda $1 \in I$. Se para quaisquer $a \in A$ e $b \in I$, tivermos $a b \in I$ e $b a \in I$, dizemos que $I$ é um ideal bilateral de $A$.

Se $I$ é um ideal bilateral de $A$, a álgebra quociente de $A$ por $I$ é denotada por $A / I$. A subálgebra gerada por um conjunto $S \subseteq A$ é a menor subálgebra de $A$ que contém $S$. Analogamente se define o ideal bilateral de $A$ gerado por um conjunto $S \subseteq A$. 
Exemplo 1.1. Seja $X=\left\{x_{0}, x_{1}, x_{2}, \ldots\right\}$ um conjunto enumerável de variáveis. $A$ álgebra $F\langle X\rangle$ com base formada por 1 e pelos monômios $x_{i_{1}} \ldots x_{i_{n}}, x_{i_{s}} \in X$, com multiplicação

$$
\left(x_{i_{1}} \ldots x_{i_{m}}\right)\left(x_{j_{1}} \ldots x_{j_{n}}\right)=x_{i_{1}} \ldots x_{i_{m}} x_{j_{1}} \ldots x_{j_{n}}
$$

é chamada álgebra associativa livre (unitária), livremente gerada por X. Os elementos de $F\langle X\rangle$ são chamados de polinômios.

De agora em diante e à menos que se faça menção contrária, a palavra "álgebra" significará "álgebra associativa unitária". Omitiremos também o termo "sobre $F$ " quando não houver dúvida sobre qual corpo a álgebra está definida.

Seja $f=f\left(x_{1}, \ldots, x_{n}\right) \in F\langle X\rangle$ um polinômio e $A$ uma álgebra. Dizemos que $f$ é uma identidade polinomial para $A$ se

$$
f\left(a_{1}, \ldots, a_{n}\right)=0, \text { para quaisquer } a_{1}, \ldots, a_{n} \in A
$$

No caso afirmativo, se $f$ é um polinômio não nulo de $F\langle X\rangle$, dizemos que $A$ é uma $P I$-álgebra (do inglês Polynomial Identity). É comum escrever " $f=0$ " para dizer que $f$ é uma identidade polinomial.

Sejam $a_{1}, \ldots, a_{n}$ elementos de uma álgebra. O comutador de comprimento 2 é definido por $\left[a_{1}, a_{2}\right]=a_{1} a_{2}-a_{2} a_{1}$. O comutador de comprimento $n(n \geq 3)$ é definido recursivamente por

$$
\left[a_{1}, \ldots, a_{n-1}, a_{n}\right]=\left[\left[a_{1}, \ldots, a_{n-1}\right], a_{n}\right]
$$

Exemplo 1.2. Uma álgebra A é comutativa se, e somente se, satisfaz a identidade polinomial $\left[x_{1}, x_{2}\right]=0$.

Exemplo 1.3. Seja A uma álgebra de dimensão $<n(n \in \mathbb{N})$. Então A satisfaz a identidade padrão de grau $n$

$$
s_{n}\left(x_{1}, \ldots, x_{n}\right)=\sum_{\sigma \in S_{n}}(-1)^{\sigma} x_{\sigma(1)} \ldots x_{\sigma(n)}
$$

onde $S_{n}$ é o grupo das permutações de $\{1,2, \ldots, n\}$ e $(-1)^{\sigma}$ é o sinal de $\sigma$.

Seja $M_{n}(F)$ a álgebra das matrizes $n \times n$ com entradas no corpo $F$. Essa álgebra possui dimensão $n^{2}$ e portanto satisfaz a identidade padrão de grau $n^{2}+1$. Em 1950, Amitsur e Levitski[1] provaram, por métodos puramente combinatórios, o seguinte resultado: 
Teorema $1.4([1])$. A álgebra $M_{n}(F)$ das matrizes $n \times n$ satisfaz a identidade padrão de grau $2 n$

$$
s_{2 n}\left(x_{1}, \ldots, x_{2 n}\right)=\sum_{\sigma \in S_{2 n}}(-1)^{\sigma} x_{\sigma(1)} \ldots x_{\sigma(2 n)} .
$$

Sejam $F$ um corpo infinito de característica $\neq 2$ e $V$ um espaço vetorial sobre $F$ com base enumerável $e_{1}, e_{2}, \ldots$. A álgebra de Grassmann infinitamente gerada $e$ unitária de $V$, denotada por $E$, é a álgebra associativa gerada por $1, e_{1}, e_{2}, \ldots$ e com relações

$$
e_{i} e_{j}=-e_{j} e_{i}
$$

para quaisquer $i, j \in \mathbb{N}$. Formalmente, $E$ é o quociente da álgebra livre $F\langle X\rangle$ pelo ideal bilateral gerado por todos os polinômios $x_{i} x_{j}+x_{j} x_{i}, i, j \in \mathbb{N}$. Analogamente se define $E_{n}$, a álgebra de Grassmann (unitária) finitamente gerada por $1, e_{1}, e_{2}, \ldots, e_{n}$.

Como um espaço vetorial, $E$ possui uma base formada por 1 e por todos os monômios

$$
e_{i_{1}} e_{i_{2}} \ldots e_{i_{k}}, \quad i_{1}<i_{2}<\ldots<i_{k}, i_{s}, k \in \mathbb{N}
$$

Exemplo 1.5. A álgebra de Grassmann E satisfaz a identidade polinomial $\left[x_{1}, x_{2}, x_{3}\right]=0$.

De fato, como o comutador $\left[x_{1}, x_{2}, x_{3}\right]$ é linear em cada entrada e a álgebra de Grassmann $E$ é gerada como espaço vetorial pelos elementos (1-4), é suficiente mostrar que $[a, b, c]=0$ para quaisquer elementos $a, b$ e $c$ da forma (1-4). Se $a$ ou $b$ é formado por um número par de símbolos $e_{s}$, então usando a relação $e_{i} e_{j}=-e_{j} e_{i}$, vemos que $a$ comuta com $b, \operatorname{logo}[a, b, c]=0$. Se $a$ e $b$ são formados por um número ímpar de símbolos $e_{s}$, então $[a, b]=a b-b a$ onde $a b$ e $b a$ são formados por um número par de símbolos $e_{s}$. Pelo caso anterior

$$
[[a, b], c]=[a b-b a, c]=[a b, c]-[b a, c]=0 .
$$

Portanto $[a, b, c]=0$ para quaisquer $a, b$ e $c$ da forma (1-4).

Sejam $F$ um corpo e $G$ um grupo finito. A álgebra do grupo $G$, denotada por $F G$, é o espaço vetorial com base $\{g: g \in G\}$ e multiplicação definida por

$$
\left(\sum_{g \in G} \alpha_{g} g\right)\left(\sum_{h \in G} \beta_{h} h\right)=\sum_{g, h \in G} \alpha_{g} \beta_{h} g h
$$

onde gh é o produto em $G$.

Exemplo 1.6. Seja $\mathcal{G}$ o grupo gerado pelos elementos $a_{1}, a_{2}, \ldots$ satisfazendo as relações $a_{i}^{2}=1,\left(\left(a_{i}, a_{j}\right), a_{k}\right)=1, i, j, k \in \mathbb{N}$, onde $(a, b)=a^{-1} b^{-1} a b$. Sejam $F$ um 
corpo de característica 2 e $F \mathcal{G}$ a álgebra do grupo $\mathcal{G}$. Defina $g_{i j}=\left(a_{i}, a_{j}\right)+1$ e seja I o ideal bilateral de $F \mathcal{G}$ gerado pelos elementos

$$
g_{i j} g_{k l}+g_{i k} g_{j l}, i, j, k, l \in \mathbb{N} \text {. }
$$

É bem conhecido que $F G / I$ satisfaz a identidade polinomial $\left[x_{1}, x_{2}, x_{3}\right]=0$ (veja [12, Lema 2.5], [27, Lema 2.1], [28, Exemplo 3.8]) e não satisfaz a identidade polinomial $\left[x_{1}, x_{2}\right] \ldots\left[x_{2 n-1}, x_{2 n}\right]=0$ para cada $n \in \mathbb{N}$ (veja [12, Lema 2.6]).

Lembremos que uma transformação linear $\varphi: A \longrightarrow B$ entre duas álgebras é um homomorfismo de álgebras se

$$
\varphi(a b)=\varphi(a) \varphi(b), \quad \text { para quaisquer } a, b \in A .
$$

Um homomorfismo $\varphi: A \longrightarrow A$ é chamado endomorfismo da álgebra $A$. A seguinte proposição torna fácil a construção de certos endomorfismos de $F\langle X\rangle$ (veja [13], pág. $9)$.

Proposição 1.7. A álgebra $F\langle X\rangle$ satisfaz a seguinte propriedade universal: se A é uma álgebra, então toda aplicação $\varphi: X \longrightarrow A$ pode ser estendida de maneira única à um homomorfismo $\tilde{\varphi}: F\langle X\rangle \longrightarrow A$.

Um ideal bilateral $I$ de $F\langle X\rangle$ é um $T$-ideal se $\varphi(I) \subseteq I$ para todos endomorfismos $\varphi$ de $F\langle X\rangle$. O $T$-ideal gerado por um conjunto $S \subseteq F\langle X\rangle$, denotado por $\langle S\rangle^{T}$, é o menor $T$-ideal de $F\langle X\rangle$ que contém $S$. Quando $S$ é finito, dizemos que o $T$-ideal é finitamente gerado.

Seja $\operatorname{Id}(A)$ o conjunto de todas as identidades polinomiais de uma álgebra $A$. Observe que $I d(A)$ é um ideal bilateral de $F\langle X\rangle$. Mais ainda $I d(A)$ é um $T$-ideal de $A$. De fato, seja $f\left(x_{1}, \ldots, x_{n}\right)$ uma identidade polinomial de $A$ e $\varphi$ um endomorfismo de $F\langle X\rangle$. É claro que $f\left(g_{1}, \ldots, g_{n}\right)$ é ainda uma identidade de $A$ para quaisquer $g_{1}, \ldots, g_{n} \in F\langle X\rangle$. Como

$$
\varphi\left(f\left(x_{1}, \ldots, x_{n}\right)\right)=f\left(\varphi\left(x_{1}\right), \ldots, \varphi\left(x_{n}\right)\right),
$$

segue que $\varphi\left(f\left(x_{1}, \ldots, x_{n}\right)\right)$ é uma identidade de $A$. Logo $I d(A)$ é um $T$-ideal de $F\langle X\rangle$.

Todo $T$-ideal é formado pelas identidades polinomiais de alguma álgebra $A$. De fato, se $I$ é um $T$-ideal qualquer de $F\langle X\rangle$, não é difícil verificar que $I=I d(A)$ onde $A=F\langle X\rangle / I$.

O grau de um monômio $u=x_{i_{1}} \ldots x_{i_{n}} \in F\langle X\rangle$, denotado por $\operatorname{deg} u$, é definido por seu comprimento, isto é $\operatorname{deg} u=n$. Assim $\operatorname{deg} f$, o grau de um polinômio $f \in F\langle X\rangle$, é definido como sendo o grau máximo dentre os monômios de $f$. Se $x_{i}$ é uma variável 
do monômio $u$, o grau de $u$ em $x_{i}$, denotado por $\operatorname{deg}_{x_{i}} u$, é o número de ocorrências de $x_{i}$ em $u$.

Um polinômio $f=f\left(x_{1}, \ldots, x_{n}\right)$ é homogêneo de grau $m_{i}$ em $x_{i}$, se todos os monômios de $f$ possuem grau $m_{i}$ em $x_{i}$; e é multi-homogêneo de multigrau $\left(m_{1}, \ldots, m_{n}\right)$ se $f$ for homogêneo de grau $m_{i}$ em $x_{i}$, para cada $i=1, \ldots, n$. Seja $f=f\left(x_{1}, \ldots, x_{n}\right)$ um polinômio qualquer de $F\langle X\rangle$, podemos sempre escrever

$$
f=\sum_{m_{1} \geq 0, \ldots, m_{n} \geq 0} f^{\left(m_{1}, \ldots, m_{n}\right)}
$$

onde $f^{\left(m_{1}, \ldots, m_{n}\right)}$ é a soma de todos os monômios de $f$ com multigrau $\left(m_{1}, \ldots, m_{n}\right)$. Os polinômios $f^{\left(m_{1}, \ldots, m_{n}\right)}$ são chamados de componentes multi-homogêneas de $f$.

Seja $f=f\left(x_{1}, \ldots, x_{n}\right) \in F\langle X\rangle$ um polinômio homogêneo de grau $m$ em $x_{1}$. O polinômio

$$
h=h\left(y_{1}, \ldots, y_{m}, x_{2}, \ldots, x_{n}\right)
$$

formado pela soma de todos os monômios $g$ de $f\left(y_{1}+\cdots+y_{m}, x_{2}, \ldots, x_{n}\right)$ tais que $\operatorname{deg}_{y_{i}} g=1$, para cada $i=1, \ldots, m$ é chamado de linearização total de $f$ em $x_{1}$.

É claro que $h$ é linear nas variáveis $y_{1}, \ldots, y_{m}$. Outra propriedade importante de $h$ é a seguinte igualdade:

$$
h\left(x_{1}, \ldots, x_{1}, x_{2}, \ldots, x_{n}\right)=m ! f\left(x_{1}, \ldots, x_{n}\right) .
$$

Seja $I$ um $T$-ideal de $F\langle X\rangle$ e $f \in I$ um polinômio qualquer. Se todas as componentes multi-homogêneas de $f$ ainda pertencerem a $I$, dizemos que $I$ é um T-ideal multi-homogêneo.

A proposição seguinte é bem conhecida, veja [13, Proposição 4.2.3] e [19, Teorema 1.3.2].

Proposição 1.8. Seja F um corpo infinito. Então todo T-ideal é gerado por seus polinômios multi-homogêneos.

Dizemos que um polinômio $f\left(x_{1}, \ldots, x_{n}\right) \in F\langle X\rangle$ é multilinear se ele é multihomogêneo de multigrau $(1, \ldots, 1)$. A proposição seguinte também é bem conhecida, veja [13, Proposição 4.2.3] e [19, Teorema 1.3.8].

Proposição 1.9. Seja F um corpo de característica 0 . Então todo T-ideal é gerado por seus polinômios multilineares.

Seja $T^{(n)}$ o ideal bilateral de $F\langle X\rangle$ gerado por todos os comutadores $\left[a_{1}, a_{2}, \ldots, a_{n}\right]$, onde $a_{i} \in F\langle X\rangle$. 
É um fato bem conhecido (veja por exemplo [13]) que se $I$ for um $T$-ideal gerado por um conjunto $S \subseteq F\langle X\rangle$, então todo elemento de $I$ é uma combinação linear de polinômios da forma

$$
a f\left(b_{1}, \ldots, b_{n}\right) c
$$

onde $a, b_{1}, \ldots, b_{n}, c$ pertencem a $F\langle X\rangle$ e $f\left(x_{1}, \ldots, x_{n}\right)$ pertence a $S$.

Existe uma situação particular (e bem conhecida) da Proposição 1.8 na qual o corpo $F$ não precisa ser infinito.

Proposição 1.10. Sejam $F$ um corpo qualquer e $f=f\left(x_{1}, \ldots, x_{n}\right) \in F\langle X\rangle$ um polinômio multilinear. Então o T-ideal gerado por $f$ é multi-homogêneo. Em particular, $T^{(n)}$ é multi-homogêneo.

Demonstração. Seja $g \in\langle f\rangle^{T}$. Então $g$ é uma combinação linear de polinômios da forma

$$
a f\left(b_{1}, \ldots, b_{n}\right) c
$$

onde $a, c$ e $b_{s}(s=1, \ldots, n)$ pertencem a $F\langle X\rangle$. Escreva os polinômios $a, c$ e $b_{s}(s=$ $1, \ldots, n)$ como soma de suas componentes multi-homogêneas:

$$
a=\sum a^{\left(i_{1}, \ldots, i_{n}\right)}, c=\sum c^{\left(j_{1}, \ldots, j_{n}\right)}, b_{s}=\sum b_{s}^{\left(k_{1}, \ldots, k_{n}\right)}
$$

Como $f$ é multilinear, segue de (1-5) que $g$ é uma combinação linear de polinômios da forma

$$
a^{\left(i_{1}, \ldots, i_{n}\right)} f\left(b_{1}^{\left(r_{1}, \ldots, r_{n}\right)}, \ldots, b_{n}^{\left(k_{1}, \ldots, k_{n}\right)}\right) c^{\left(j_{1}, \ldots, j_{n}\right)}
$$

Segue também da multilinearidade de $f$ que cada polinômio $f\left(b_{1}^{\left(r_{1}, \ldots, r_{n}\right)}, \ldots, b_{n}^{\left(k_{1}, \ldots, k_{n}\right)}\right)$ é multi-homogêneo e assim cada polinômio de (1-7) é multi-homogêneo. Portanto, cada componente multi-homogênea de $g$ é uma combinação linear de polinômios de (1-7), isto é, cada componente multi-homogênea de $g$ pertence a $\langle f\rangle^{T}$. Isso mostra que $\langle f\rangle^{T}$ é multi-homogêneo. É fácil ver que $T^{(n)}$ é gerado como $T$-ideal pelo comutador $\left[x_{1}, \ldots, x_{n}\right]$, que é um polinômio multilinear. Logo $T^{(n)}$ é multihomogêneo.

Dizemos que $f \in F\langle X\rangle$ é um polinômio próprio se ele for uma combinação linear de produtos de comutadores da forma

$$
\left[x_{i_{1}}, \ldots, x_{i_{r}}\right] \ldots\left[x_{j_{1}}, \ldots, x_{j_{s}}\right]
$$

Assumimos que 1 é também um polinômio próprio. O conjunto dos polinômios próprios de $F\langle X\rangle$ será denotado por $B$. Denotaremos também

$$
B_{n}=B \cap F\left\langle x_{1}, \ldots, x_{n}\right\rangle, n \in \mathbb{N},
$$


isto é, $B_{n}$ é o conjunto dos polinômios próprios de $F\langle X\rangle$ em $n$ variáveis. O resultado seguinte é devido a Drensky [14]. Veja também [13, Teorema 4.3.11].

Teorema 1.11 (veja [14], Lema 2.4). Seja A uma PI-álgebra sobre um corpo infinito F. Se

$$
w_{j}\left(x_{1}, \ldots, x_{n}\right)+\left(B_{n} \cap I d(A)\right), j=1,2, \ldots
$$

é uma base para o espaço vetorial $B_{n}(A)=B_{n} /\left(B_{n} \cap I d(A)\right)$, então o espaço vetorial $F_{n}(A)=F\left\langle X_{n}\right\rangle /\left(F\left\langle X_{n}\right\rangle \cap I d(A)\right)$ possui uma base

$$
x_{1}^{r_{1}} \ldots x_{n}^{r_{n}} w_{j}\left(x_{1}, \ldots, x_{n}\right), r_{i} \geq 0, j=1,2, \ldots
$$

Um pequeno comentário: a hipótese do corpo $F$ ser infinito no enunciado do Teorema 1.11 é para garantir que $I d(A)$ seja um $T$-ideal multi-homogêneo (conforme a Proposição 1.8). Assim a demonstração do teorema funciona apenas com a hipótese de $I d(A)$ ser multi-homogêneo. Em particular, como $\operatorname{Id}\left(Q_{n}\right)=T^{(n)}$ é multi-homogêneo (Proposição 1.10), o teorema vale quando $A=Q_{n}=F\langle X\rangle / T^{(n)}$ e $F$ é um corpo qualquer. Todas as referências ao Teorema 1.11 estarão sendo usadas nesse caso particular.

Dizemos que uma álgebra $A$ é uma álgebra de Lie se para quaisquer $a, b, c \in A$,

$$
\begin{aligned}
& a a=0, \\
& (a b) c+(b c) a+(c a) b=0 .
\end{aligned}
$$

Quando $A$ é uma álgebra de Lie, é usual denotar o produto $a b$ por $[a, b]$ (colchete de Lie). Assim, as propriedades (1-1)-(1-3) significam que [, ] é bilinear e as propriedades (1-8)-(1-9) são escritas como

$$
\begin{aligned}
& {[a, a]=0, \quad(\text { lei anti-comutativa })} \\
& {[[a, b], c]+[[b, c], a]+[[c, a], b]=0 \quad \text { (identidade de Jacobi). }}
\end{aligned}
$$

Pela lei anti-comutativa e pela bilinearidade do colchete de Lie temos

$$
0=[a+b, a+b]=[a, a]+[a, b]+[b, a]+[b, b]=[a, b]+[b, a] .
$$

Consequentemente $[a, b]=-[b, a]$, para quaisquer $a, b \in A$.

Exemplo 1.12. Em uma álgebra (associativa) $A$, defina o seguinte produto $[a, b]=$ $a b-b a, a, b \in A$. É um trabalho rotineiro mostrar que A é uma álgebra de Lie com esse novo produto. 
A álgebra $Q_{n}=F\langle X\rangle / T^{(n)}$ é chamada álgebra associativa Lie nilpotente universal de classe $n-1$ ou ainda álgebra relativamente livre na classe de álgebras associativas definidas pela identidade $\left[x_{1}, \ldots, x_{n}\right]=0$.

\subsection{Polinômios centrais e $T$-subespaços}

Seja $Z(A)$ o centro de uma álgebra $A$, isto é $Z(A)=\{a \in A: a b=$ ba para todo $b \in A\}$. Dizemos que $f\left(x_{1}, \ldots, x_{n}\right) \in F\langle X\rangle$ é um polinômio central de $A$ se

$$
f\left(a_{1}, \ldots, a_{n}\right) \in Z(A) \text { para quaisquer } a_{1}, \ldots, a_{n} \in A .
$$

Um subespaço vetorial $I$ de $F\langle X\rangle$ é chamado $T$-subespaço se $\varphi(I) \subseteq I$ para todo endomorfismo $\varphi$ de $F\langle X\rangle$. Uma subálgebra de $F\langle X\rangle$ que é também um $T$ subespaço é chamada $T$-subálgebra de $F\langle X\rangle$.

O conjunto $C(A)$ de todos os polinômios centrais de $A$ forma um subespaço vetorial de $F\langle X\rangle$. Mais ainda $C(A)$ é uma $T$-subálgebra de $F\langle X\rangle$. De fato, segue imediatamente da definição de polinômio central que se $f\left(x_{1}, \ldots, x_{n}\right) \in C(A)$, então $f\left(g_{1}, \ldots, g_{n}\right) \in C(A)$ para quaisquer polinômios $g_{1}, \ldots, g_{n} \in F\langle X\rangle$. Como

$$
\varphi\left(f\left(x_{1}, \ldots, x_{n}\right)\right)=f\left(\varphi\left(x_{1}\right), \ldots, \varphi\left(x_{n}\right)\right)
$$

segue que $\varphi\left(f\left(x_{1}, \ldots, x_{n}\right)\right)$ pertence a $C(A)$ para qualquer endomorfismo $\varphi$ de $F\langle X\rangle$. Portanto $C(A)$ é um $T$-subespaço de $F\langle X\rangle$. Como $Z(A)$ é uma subálgebra de $A$, segue imediatamente da definição de polinômio central que $C(A)$ é uma subálgebra de $F\langle X\rangle$. Para referência futura no texto vamos escrever esse resultado (que é bem conhecido) na forma de uma proposição.

Proposição 1.13. $C(A)$ é uma $T$-subálgebra de $F\langle X\rangle$.

Ao contrário de $T$-ideais, o estudo dos $T$-subespaços não se reduz ao estudo dos polinômios centrais. Mais precisamente, existem $T$-subespaços $I$ de $F\langle X\rangle$ para os quais não existe uma álgebra $A$ tal que $I=C(A)$ (veja [20, Observação 1]).

Um $T$-subespaço $I$ é gerado por um conjunto $S \subseteq F\langle X\rangle$ se $I$ for o menor $T$-subespaço de $F\langle X\rangle$ que contém $S$. No caso afirmativo escrevemos $I=\langle S\rangle^{T S}$. Se $S$ for finito, dizemos que $I$ é um $T$-subespaço finitamente gerado.

Seja $I$ um $T$-subespaço de $F\langle X\rangle$ e $f \in I$ um polinômio qualquer. Se todas as componentes multi-homogêneas de $f$ ainda pertencerem a $I$, dizemos que $I$ é um T-subespaço multi-homogêneo. 
As duas proposições seguintes são análogas a Proposição 1.8 e a Proposição 1.9, respectivamente. As demonstrações destas são também idênticas às demonstrações daquelas, veja [13, 19].

Proposição 1.14. Seja F um corpo infinito. Então todo T-subespaço é gerado por seus polinômios multi-homogêneos.

Proposição 1.15. Seja F um corpo de característica 0. Então todo T-subespaço é gerado por seus polinômios multilineares.

À seguir, um caso particular (e bem conhecido) da Proposição 1.14 que será muito utilizado nesta tese.

Proposição 1.16. O T-subespaço $C\left(Q_{n}\right)$ é multi-homogêneo.

Demonstração. Seja $f=f\left(x_{1}, \ldots, x_{k}\right) \in C\left(Q_{n}\right)$. Escreva $f=\sum f^{\left(m_{1}, \ldots, m_{k}\right)}$, onde cada $f^{\left(m_{1}, \ldots, m_{k}\right)}$ é uma componente multi-homogênea de $f$ com multigrau $\left(m_{1}, \ldots, m_{k}\right)$. Observe que $g=\left[f, x_{k+1}\right]=\sum\left[f^{\left(m_{1}, \ldots, m_{k}\right)}, x_{k+1}\right]$ pertence a $T^{(n)}$ e cada polinômio $\left[f^{\left(m_{1}, \ldots, m_{k}\right)}, x_{k+1}\right]$ é uma componente multi-homogênea de $g$. Como $T^{(n)}$ é multihomogêneo (Proposição 1.10), segue que $\left[f^{\left(m_{1}, \ldots, m_{k}\right)}, x_{k+1}\right]$ pertence a $T^{(n)}$, isto é $f^{\left(m_{1}, \ldots, m_{k}\right)}$ pertence a $C\left(Q_{n}\right)$. Portanto $C\left(Q_{n}\right)$ é multi-homogêneo.

O resultado seguinte é bem conhecido e será usado diversas vezes no texto.

Lema 1.17. As seguintes igualdades são válidas em $F\langle X\rangle$

(i) $\left[a_{1} a_{2}, a_{3}, a_{4}\right]=a_{1}\left[a_{2}, a_{3}, a_{4}\right]+\left[a_{1}, a_{3}\right]\left[a_{2}, a_{4}\right]+\left[a_{1}, a_{4}\right]\left[a_{2}, a_{3}\right]+\left[a_{1}, a_{3}, a_{4}\right] a_{2}$,

(ii) $\left[a_{1} a_{2} \ldots a_{n}, a\right]=\sum_{i=1}^{n} a_{1} \ldots a_{i-1}\left[a_{i}, a\right] a_{i+1} \ldots a_{n}$.

Demonstração. Para provar o item (i) usamos duas vezes a igualdade $[a b, c]=$ $a[b, c]+[a, c] b$. Para o item (ii), a mesma igualdade e indução sobre $n$.

\subsection{Relações na álgebra $Q_{3}$}

O objetivo desta seção é dar uma descrição da álgebra $Q_{3}=F\langle X\rangle / T^{(3)}$. O lema seguinte é bem conhecido, veja por exemplo [2, 9, 13, 18, 20, 32].

Lema 1.18. Seja F um corpo qualquer. Então $T^{(3)}$ contém os seguintes polinômios

(i) $\left[x_{1}, x_{2}\right]\left[x_{3}, x_{4}\right]+\left[x_{1}, x_{3}\right]\left[x_{2}, x_{4}\right]$,

(ii) $\left[x_{1}, x_{2}\right]\left[x_{2}, x_{3}\right]$. 
Demonstração. Vamos provar (i). Pelo Lema 1.17 (i), temos

$$
\begin{aligned}
{\left[x_{1} x_{2}, x_{3}, x_{4}\right] } & =x_{1}\left[x_{2}, x_{3}, x_{4}\right]+\left[x_{1}, x_{3}\right]\left[x_{2}, x_{4}\right]+\left[x_{1}, x_{4}\right]\left[x_{2}, x_{3}\right]+\left[x_{1}, x_{3}, x_{4}\right] x_{2} \\
& =x_{1}\left[x_{2}, x_{3}, x_{4}\right]+\left[x_{1}, x_{3}, x_{4}\right] x_{2}-\left[x_{1}, x_{3}\right]\left[x_{4}, x_{2}\right]-\left[x_{1}, x_{4}\right]\left[x_{3}, x_{2}\right]
\end{aligned}
$$

Como $\left[x_{1} x_{2}, x_{3}, x_{4}\right], x_{1}\left[x_{2}, x_{3}, x_{4}\right]$ e $\left[x_{1}, x_{3}, x_{4}\right] x_{2}$ pertencem a $T^{(3)}$, segue que $\left[x_{1}, x_{3}\right]\left[x_{4}, x_{2}\right]+\left[x_{1}, x_{4}\right]\left[x_{3}, x_{2}\right]$ pertence a $T^{(3)}$. Reenumerando as variáveis vemos que o polinômio (i) pertence a $T^{(3)}$. Agora vejamos (ii). Usando a igualdade $[a b, c]=a[b, c]+[a, c] b$, temos

$$
\begin{aligned}
{\left[x_{1}, x_{2}\right]\left[x_{1}, x_{3}\right] } & =\left[\left[x_{1}, x_{2}\right] x_{1}, x_{3}\right]-\left[x_{1}, x_{2}, x_{3}\right] x_{1} \\
& =\left[\left[x_{1}, x_{2} x_{1}\right], x_{3}\right]-\left[x_{1}, x_{2}, x_{3}\right] x_{1}
\end{aligned}
$$

Logo $\left[x_{1}, x_{2}\right]\left[x_{1}, x_{3}\right]$ pertence a $T^{(3)}$.

Sejam $a_{1}, a_{2}, a_{3}, a_{4}$ polinômios quaisquer de $F\langle X\rangle$. Defina

$$
\omega\left(a_{1}, a_{2}, a_{3}, a_{4}\right)=\left[a_{1}, a_{2}\right]\left[a_{3}, a_{4}\right]+\left[a_{1}, a_{3}\right]\left[a_{2}, a_{4}\right] .
$$

Antes de prosseguir, vejamos algumas propriedades do polinômio $\omega$.

Lema 1.19. Seja $F$ um corpo qualquer. As seguintes igualdades são válidas em $F\langle X\rangle$ para quaisquer $a_{1}, \ldots, a_{5} \in F\langle X\rangle$.

(i) $\omega\left(a_{1} a_{2}, a_{3}, a_{4}, a_{5}\right)=a_{1} \omega\left(a_{2}, a_{3}, a_{4}, a_{5}\right)+a_{2} \omega\left(a_{1}, a_{3}, a_{4}, a_{5}\right)+\left[a_{1}, a_{3}, a_{2}\right]\left[a_{4}, a_{5}\right]+$ $\left[a_{1}, a_{4}, a_{2}\right]\left[a_{3}, a_{5}\right]$,

(ii) $\omega\left(a_{1}, a_{2} a_{3}, a_{4}, a_{5}\right)=a_{2} \omega\left(a_{1}, a_{3}, a_{4}, a_{5}\right)+\omega\left(a_{1}, a_{2}, a_{4}, a_{5}\right) a_{3}+\left[a_{1}, a_{4}, a_{2}\right]\left[a_{3}, a_{5}\right]+$ $\left[a_{1}, a_{2}\right]\left[a_{5}, a_{4}, a_{3}\right]$,

(iii) $\omega\left(a_{1}, a_{2}, a_{3}, a_{4} a_{5}\right)=a_{4} \omega\left(a_{1}, a_{2}, a_{3}, a_{5}\right)+\omega\left(a_{1}, a_{2}, a_{3}, a_{4}\right) a_{5}+\left[a_{1}, a_{2}, a_{4}\right]\left[a_{3}, a_{5}\right]+$ $\left[a_{1}, a_{3}, a_{4}\right]\left[a_{2}, a_{5}\right]$.

Demonstração. Basta usar a definição de $\omega$ em cada membro esquerdo de cada um dos itens.

A proposição seguinte é bem conhecida. Veja por exemplo [2, 9, 13, 18, 20, $32]$.

Proposição 1.20. Seja $F$ um corpo qualquer. Então $T^{(3)}$ é gerado como ideal bilateral de $F\langle X\rangle$ pelos polinômios

(i) $\left[x_{i_{1}}, x_{i_{2}}, x_{i_{3}}\right] \quad\left(x_{i_{s}} \in X\right)$,

(ii) $\left[x_{i_{1}}, x_{i_{2}}\right]\left[x_{i_{3}}, x_{i_{4}}\right]+\left[x_{i_{1}}, x_{i_{3}}\right]\left[x_{i_{2}}, x_{i_{4}}\right]\left(x_{i_{s}} \in X\right)$. 
Demonstração. Seja $I$ o ideal bilateral de $F\langle X\rangle$ gerado pelos polinômios (i) e (ii). Devemos mostrar que $T^{(3)}=I$. Pelo Lema 1.18, temos $I \subseteq T^{(3)}$. Resta mostrar que $T^{(3)} \subseteq I$. Observe que $T^{(3)}$ é gerado como ideal bilateral de $F\langle X\rangle$ pelos polinômios da forma

$$
\left[a_{1}, a_{2}, a_{3}\right]
$$

onde os $a_{i}$ são monômios quaisquer em $F\langle X\rangle$. Assim é suficiente mostrar que os polinômios da forma (1-10) pertencem a $I$. Faremos a prova por indução, e para ela funcionar, precisamos mostrar também que $I$ contém todos os polinômios da forma

$$
\omega\left(a_{1}, a_{2}, a_{3}, a_{4}\right)
$$

onde $\omega\left(x_{1}, x_{2}, x_{3}, x_{4}\right)=\left[x_{1}, x_{2}\right]\left[x_{3}, x_{4}\right]+\left[x_{1}, x_{3}\right]\left[x_{2}, x_{4}\right]$ e os $a_{i}$ são monômios quaisquer de $F\langle X\rangle$. A prova será por indução sobre $m=\operatorname{deg} f$, onde $f$ é um polinômio da forma (1-10) ou da forma (1-11).

É claro que $\operatorname{deg} f \geq 3$. Se $\operatorname{deg} f=3$, então $f$ é da forma (1-10) com cada $a_{i}$ de grau igual a 1 , isto é, $f$ é da forma (i) e portanto $f$ pertence a $I$. Assim, a base da indução é $m=3$. Para o passo de indução suponha que $\operatorname{deg} f=m>3$ e que todos os polinômios de (1-10)-(1-11) de grau menor do que $m$ pertençam a $I$.

Suponha primeiramente que $f$ é um polinômio da forma (1-11) e vamos denotá-lo por $\omega$. Se $\operatorname{deg} \omega=4$, então cada $a_{i}$ possui grau igual a 1 . Assim $\omega$ é um polinômio de (ii) e temos $\omega \in I$. Podemos então supor que $\operatorname{deg} \omega=m>4$. Então $a_{i}=a_{i}^{\prime} a_{i}^{\prime \prime} \operatorname{com} \operatorname{deg} a_{i}^{\prime}, \operatorname{deg} a_{i}^{\prime \prime}<\operatorname{deg} a_{i}$ para algum $i, 1 \leq i \leq 4$. Como $\omega\left(a_{1}, a_{2}, a_{3}, a_{4}\right)=$ $\omega\left(a_{1}, a_{3}, a_{2}, a_{4}\right)$ é suficiente considerar os casos $a_{1}=a_{1}^{\prime} a_{1}^{\prime \prime}, a_{2}=a_{2}^{\prime}, a_{2}^{\prime \prime}$ e $a_{4}=a_{4}^{\prime} a_{4}^{\prime \prime}$. Pela Proposição 1.19, temos

$$
\begin{aligned}
\omega\left(a_{1}^{\prime} a_{1}^{\prime \prime}, a_{2}, a_{3}, a_{4}\right) & =a_{1}^{\prime} \omega\left(a_{1}^{\prime \prime}, a_{2}, a_{3}, a_{4}\right)+a_{1}^{\prime \prime} \omega\left(a_{1}^{\prime}, a_{2}, a_{3}, a_{4}\right)+ \\
& +\left[a_{1}^{\prime}, a_{2}, a_{1}^{\prime \prime}\right]\left[a_{3}, a_{4}\right]+\left[a_{1}^{\prime}, a_{3}, a_{1}^{\prime \prime}\right]\left[a_{2}, a_{4}\right]
\end{aligned}
$$

Como os graus dos polinômios $\left[a_{1}^{\prime}, a_{2}, a_{1}^{\prime \prime}\right],\left[a_{1}^{\prime}, a_{3}, a_{1}^{\prime \prime}\right], \omega\left(a_{1}^{\prime \prime}, a_{2}, a_{3}, a_{4}\right)$ e $\omega\left(a_{1}^{\prime}, a_{2}, a_{3}, a_{4}\right)$ são menores do que $m$, segue da hipótese de indução que todos esses polinômios pertencem a $I$. Logo também pertencem a $I$ todos os polinômios do lado direito da igualdade em (1-12). Segue assim que o polinômio $\omega\left(a_{1}^{\prime} a_{1}^{\prime \prime}, a_{2}, a_{3}, a_{4}\right)$ pertence a $I$. Novamente pela Proposição 1.19 temos

$$
\begin{aligned}
\omega\left(a_{1}, a_{2}^{\prime} a_{2}^{\prime \prime}, a_{3}, a_{4}\right) & =a_{2}^{\prime} \omega\left(a_{1}, a_{2}^{\prime \prime}, a_{3}, a_{4}\right)+\omega\left(a_{1}, a_{2}^{\prime}, a_{3}, a_{4}\right) a_{2}^{\prime \prime}+ \\
& +\left[a_{1}, a_{3}, a_{2}^{\prime}\right]\left[a_{2}^{\prime \prime}, a_{4}\right]+\left[a_{1}, a_{2}^{\prime}\right]\left[a_{4}, a_{3}, a_{2}^{\prime \prime}\right], \\
\omega\left(a_{1}, a_{2}, a_{3}, a_{4}^{\prime} a_{4}^{\prime \prime}\right) & =a_{4}^{\prime} \omega\left(a_{1}, a_{2}, a_{3}, a_{4}^{\prime \prime}\right)+\omega\left(a_{1}, a_{2}, a_{3}, a_{4}^{\prime}\right) a_{4}^{\prime \prime}+ \\
& +\left[a_{1}, a_{2}, a_{4}^{\prime}\right]\left[a_{3}, a_{4}^{\prime \prime}\right]+\left[a_{1}, a_{3}, a_{4}^{\prime}\right]\left[a_{2}, a_{4}^{\prime \prime}\right],
\end{aligned}
$$


e podemos proceder de modo análogo ao caso de $\omega\left(a_{1}^{\prime} a_{1}^{\prime \prime}, a_{2}, a_{3}, a_{4}\right)$ para concluir que os polinômios da forma (1-13) e (1-15) pertencem a $I$. Mostramos assim que $\omega\left(a_{1}, a_{2}, a_{3}, a_{4}\right)$ pertence a $I$ para quaisquer monômios $a_{i} \in F\langle X\rangle$.

Suponha agora que $f$ é um polinômio da forma (1-10), isto é $f=$ $f\left(a_{1}, a_{2}, a_{3}\right)=\left[a_{1}, a_{2}, a_{3}\right]$. É claro que $\operatorname{deg} f \geq 3$. Se $\operatorname{deg} f=3$, então cada $a_{i}$ possui grau igual a 1 , isto é, $f$ é um polinômio da forma (i). Logo $f \in I$. Suponha que $\operatorname{deg} f=m>3$. Assim $a_{i}=a_{i}^{\prime} a_{i}^{\prime \prime}$ para algum $i, 1 \leq i \leq 3$. Como $f\left(a_{1}, a_{2}, a_{3}\right)=$ $-f\left(a_{2}, a_{1}, a_{3}\right)$, é suficiente considerar os casos $a_{1}=a_{1}^{\prime} a_{1}^{\prime \prime}$ e $a_{3}=a_{3}^{\prime} a_{3}^{\prime \prime}$. Seja $a_{1}=a_{1}^{\prime} a_{1}^{\prime \prime}$. Pela Proposição 1.17 (i), temos

$$
\begin{aligned}
{\left[a_{1}^{\prime} a_{1}^{\prime \prime}, a_{2}, a_{3}\right] } & \left.=a_{1}^{\prime}\left[a_{1}^{\prime \prime}, a_{2}, a_{3}\right]+\left[a_{1}^{\prime}, a_{2}, a_{3}\right] a_{1}^{\prime \prime}+\left[a_{1}^{\prime}, a_{2}\right]\left[a_{1}^{\prime \prime}, a_{3}\right]+\left[a_{1}^{\prime}, a_{3}\right]\left[a_{1}^{\prime \prime}, a_{6}\right]-15\right) \\
& =a_{1}^{\prime}\left[a_{1}^{\prime \prime}, a_{2}, a_{3}\right]+\left[a_{1}^{\prime}, a_{2}, a_{3}\right] a_{1}^{\prime \prime}-\omega\left(a_{1}^{\prime}, a_{2}, a_{3}, a_{1}^{\prime \prime}\right)
\end{aligned}
$$

Como os graus dos polinômios $\left[a_{1}^{\prime \prime}, a_{2}, a_{3}\right]$ e $\left[a_{1}^{\prime}, a_{2}, a_{3}\right]$ são menores do que $m$, segue da hipótese de indução que esses polinômios pertencem a $I$. Como já mostramos que o polinômio $\omega\left(a_{1}^{\prime}, a_{2}, a_{3}, a_{1}^{\prime \prime}\right)$ pertence a $I$, segue que todos os polinômios de (1-15) pertencem a $I$, consequentemente $\left[a_{1}^{\prime} a_{1}^{\prime \prime}, a_{2}, a_{3}\right]$ pertence a $I$. Finalmente, considere $a_{3}=a_{3}^{\prime} a_{3}^{\prime \prime}$. Temos

$$
\left[a_{1}, a_{2}, a_{3}^{\prime} a_{3}^{\prime \prime}\right]=a_{3}^{\prime}\left[a_{1}, a_{2}, a_{3}^{\prime \prime}\right]+\left[a_{1}, a_{2}, a_{3}^{\prime}\right] a_{3}^{\prime \prime}
$$

Como os polinômios $\left[a_{1}, a_{2}, a_{3}^{\prime \prime}\right]$ e $\left[a_{1}, a_{2}, a_{3}^{\prime}\right]$ possuem graus menores do que $m$, segue da hipótese de indução que eles pertencem a $I$. Logo os polinômios $a_{3}^{\prime}\left[a_{1}, a_{2}, a_{3}^{\prime \prime}\right]$ e $\left[a_{1}, a_{2}, a_{3}^{\prime}\right] a_{3}$ também pertencem a $I$ e consequentemente o polinômio $\left[a_{1}, a_{2}, a_{3}^{\prime} a_{3}^{\prime \prime}\right]$ pertence a $I$. Mostramos assim que $\left[a_{1}, a_{2}, a_{3}\right]$ pertence a $I$, para quaisquer monômios $a_{i} \in F\langle X\rangle$, portanto $T^{(3)} \subseteq I$. Como a inclusão $I \subseteq T^{(3)}$ já foi mostrada, obtemos $T^{(3)}=I$ e a demonstração está completa.

A proposição seguinte é bem conhecida, veja [7, 13, 20].

Proposição 1.21. Seja $F$ um corpo qualquer. Então o espaço vetorial $Q_{3}=$ $F\langle X\rangle / T^{(3)}$ possui uma base

$$
x_{i_{1}}^{r_{1}} \ldots x_{i_{k}}^{r_{k}}\left[x_{j_{1}}, x_{j_{2}}\right] \ldots\left[x_{j_{2 l-1}}, x_{j_{2 l}}\right]+T^{(3)},
$$

onde $k \geq 0, l \geq 0, r_{s}>0, i_{1}<\ldots<i_{k}$ e $j_{1}<\ldots<j_{2 l}$.

Demonstração. Pelo Teorema 1.11 (e o comentário logo abaixo dele) é suficiente mostrar que os elementos

$$
\left[x_{j_{1}}, x_{j_{2}}\right] \ldots\left[x_{j_{2 l-1}}, x_{j_{2 l}}\right]+\left(B \cap T^{(3)}\right), j_{1}<\ldots<j_{2 l}
$$


formam uma base para o espaço vetorial $B /\left(B \cap T^{(3)}\right)$. Mostremos primeiramente que os elementos (1-18) geram $B /\left(B \cap T^{(3)}\right)$. Seja $f=f\left(x_{1}, \ldots, x_{n}\right) \in B$ um polinômio próprio com $f \notin T^{(3)}$. Como o espaço vetorial $B$ é gerado por produtos de comutadores, podemos considerar

$$
f=\left[x_{i_{1}}, \ldots, x_{i_{k}}\right] \ldots\left[x_{j_{1}}, \ldots, x_{j_{l}}\right] .
$$

Mais ainda, como $f \notin T^{(3)}$, segue que $f$ deve ser da forma

$$
f=\left[x_{j_{1}}, x_{j_{2}}\right] \ldots\left[x_{j_{2 l-1}}, x_{j_{2 l}}\right]
$$

A igualdade $\left[x_{1}, x_{2}\right]=-\left[x_{2}, x_{1}\right]$ e a relação $\left[x_{1}, x_{2}\right]\left[x_{3}, x_{4}\right] \equiv-\left[x_{1}, x_{3}\right]\left[x_{2}, x_{4}\right]\left(\bmod T^{(3)}\right)$ (Lema $1.18(\mathrm{i})$ ) implicam que podemos trocar, módulo $B \cap T^{(3)}$, quaisquer duas variáveis de $f$ de lugar. Segue desse fato e da relação $\left[x_{1}, x_{2}\right]\left[x_{2}, x_{3}\right] \in T^{(3)}$ (Lema 1.18 (ii)), que se $f$ tiver duas variáveis iguais, então $f \in B \cap T^{(3)}$. Portanto todas as variáveis de $f$ são distintas e $f+\left(B \cap T^{(3)}\right)$ é um múltiplo escalar do elemento

$$
\left[x_{j_{1}}, x_{j_{2}}\right] \ldots\left[x_{j_{2 l-1}}, x_{j_{2 l}}\right]+\left(B \cap T^{(3)}\right), j_{1}<\ldots<j_{2 l} .
$$

Agora vamos mostrar que os elementos (1-18) são linearmente independentes. Observe que os polinômios da forma

$$
\left[x_{j_{1}}, x_{j_{2}}\right] \ldots\left[x_{j_{2 l-1}}, x_{j_{2 l}}\right], j_{1}<\ldots<j_{2 l}
$$

são multilineares e determinados por seus multi-graus. Como $T^{(3)}$ é multi-homogêneo (Proposição 1.10), basta mostrar que todo polinômio da forma (1-19) não pertence a $T^{(3)}$ ou equivalentemente, que o polinômio $b=\left[x_{1}, x_{2}\right] \ldots\left[x_{2 n-1,2 n}\right]$ não pertence a $T^{(3)}$. Para fazer isso, dividiremos o argumento em dois casos.

Caso 1: $\operatorname{char}(F) \neq 2$. Considere os $2 n$ primeiros geradores da Álgebra de Grassmann $E: e_{1}, e_{2}, \ldots, e_{2 n}$. Como

$$
\left[e_{1}, e_{2}\right] \ldots\left[e_{2 n-1}, e_{2 n}\right]=2^{n} e_{1} \ldots e_{2 n} \neq 0
$$

segue que $b$ não pertence a $I d(E)$. Logo $b$ não pertence a $T^{(3)}$.

Caso 2: $\operatorname{char}(F)=2$. Seja $F(\mathcal{G}) / I$ a álgebra do Exemplo 1.6. Como vimos $F(\mathcal{G}) / I$ satisfaz a identidade polinomial $\left[x_{1}, x_{2}, x_{3}\right]=0$ mas não satisfaz a identidade polinomial $\left[x_{1}, x_{2}\right] \ldots\left[x_{2 n-1}, x_{2 n}\right]=0$. Isso implica que $b$ não pertence a $T^{(3)}$.

Segue portanto que os elementos (1-18) formam uma base para o espaço vetorial $B /\left(B \cap T^{(3)}\right)$. Pelo Teorema 1.11, os elementos (1-17) formam uma base para o espaço vetorial $F\langle X\rangle / T^{(3)}$.

Lema $\mathbf{1 . 2 2}$ (veja [26]). Seja F um corpo de característica $p$. 
(i) Se $p \geq n-1$, então $\left[x_{0}^{p}, x_{1}\right] \equiv 0\left(\bmod T^{(n)}\right)$,

(ii) Se $p \geq n$, então $\left(x_{1} x_{2}\right)^{p} \equiv x_{1}^{p} x_{2}^{p}\left(\bmod T^{(n)}\right)$,

(iii) Se $p=2$, então $\left(x_{1} x_{2}\right)^{4} \equiv x_{1}^{4} x_{2}^{4}\left(\bmod T^{(3)}\right)$.

Demonstração. Para os itens (i) e (ii) consulte [26]. Vamos provar o item (iii). Observe que $\left(x_{1} x_{2}\right)^{2}=x_{1}^{2} x_{2}^{2}+x_{1}\left[x_{1}, x_{2}\right] x_{2}$. Assim

$$
\begin{aligned}
\left(x_{1} x_{2}\right)^{4} & =\left(x_{1} x_{2}\right)^{2}\left(x_{1} x_{2}\right)^{2}=\left(x_{1}^{2} x_{2}^{2}+x_{1}\left[x_{1}, x_{2}\right] x_{2}\right)\left(x_{1}^{2} x_{2}^{2}+x_{1}\left[x_{1}, x_{2}\right] x_{2}\right) \\
& =\left(x_{1}^{2} x_{2}^{2}\right)\left(x_{1}^{2} x_{2}^{2}\right)+\left(x_{1}^{2} x_{2}^{2}\right)\left(x_{1}\left[x_{1}, x_{2}\right] x_{2}\right)+\left(x_{1}\left[x_{1}, x_{2}\right] x_{2}\right)\left(x_{1}^{2} x_{2}^{2}\right)+ \\
& +\left(x_{1}\left[x_{1}, x_{2}\right] x_{2}\right)\left(x_{1}\left[x_{1}, x_{2}\right] x_{2}\right) .
\end{aligned}
$$

Usando a igualdade $[a b, c]=a[b, c]+[a, c] b$, temos $\left[x_{1}^{2}, x_{2}\right]=x_{1}\left[x_{1}, x_{2}\right]+\left[x_{1}, x_{2}\right] x_{1}=$ $2 x_{1}\left[x_{1}, x_{2}\right]+\left[x_{1}, x_{2}, x_{1}\right]=\left[x_{1}, x_{2}, x_{1}\right]$. Assim $x_{1}^{2}+T^{(3)}$ é central em $Q_{3}$. Como $\left[x_{1}, x_{2}\right]+$ $T^{(3)}$ também é central em $Q_{3}$, segue de (1-20) que

$$
\left(x_{1} x_{2}\right)^{4} \equiv x_{1}^{4} x_{2}^{4}+2 x_{1}^{3} x_{2}^{3}\left[x_{1}, x_{2}\right]+\left(x_{1} x_{2}\right)^{2}\left[x_{1}, x_{2}\right]\left[x_{1}, x_{2}\right] \quad\left(\bmod T^{(3)}\right)
$$

Como $\operatorname{char}(F)=2$ e $\left[x_{1}, x_{2}\right]\left[x_{1}, x_{2}\right] \in T^{(3)}$ (Lema 1.18 (ii)), obtemos $\left(x_{1} x_{2}\right)^{4} \equiv x_{1}^{4} x_{2}^{4}$ $\left(\bmod T^{(3)}\right)$.

\subsection{Polinômios centrais da álgebra $Q_{3}$}

O objetivo desta seção é dar uma descrição dos polinômios centrais da álgebra $Q_{3}=F\langle X\rangle / T^{(3)}$. Essa descrição foi obtida em 2010, independentemente por Bekh-Ochir e Rankin [3], por Brandão Jr, Koshlukov, Krasilnikov e Silva [7] e por Grishim [23]. Seguiremos exposição de [7].

Seja $F$ um corpo qualquer de característica $p>0$. Seja $q\left(x_{1}, x_{2}\right)=$ $x_{1}^{p-1}\left[x_{1}, x_{2}\right] x_{2}^{p-1}$ e para cada $n \geq 1$ defina

$$
q_{n}=q_{n}\left(x_{1}, \ldots, x_{2 n}\right)=q\left(x_{1}, x_{2}\right) q\left(x_{3}, x_{4}\right) \ldots q\left(x_{2 n-1}, x_{2 n}\right)
$$

Agora seja $E$ a álgebra de Grassmann (infinitamente gerada e unitária) sobre um corpo infinito $F$ de característica $p>2$. Foi mostrado em ([3], [7], [23]) (veja o Teorema 0.1 ) que $C(E)$, o espaço vetorial dos polinômios centrais de $E$, é gerado como $T$-subespaço de $F\langle X\rangle$ pelos polinômios

$$
x_{1}\left[x_{2}, x_{3}, x_{4}\right], x_{0}^{p}, x_{0}^{p} q_{1}, \ldots, x_{0}^{p} q_{n}, \ldots
$$


Seja $M$ o conjunto dos monômios mônicos de $F\langle X\rangle$, isto é $M=\left\{x_{i_{1}} x_{i_{2}} \ldots x_{i_{n}}\right.$ : $\left.n, i_{s} \geq 0\right\}$. A seguinte descrição mais precisa de $C(E)$ foi obtida por Deryabina e Krasilnikov [10] à partir de [7].

Teorema 1.23 (veja [7, 10]). Seja $F$ um corpo infinito de característica $p>2$. Então $C(E)$, o espaço vetorial dos polinômios centrais de E, é gerado (como espaço vetorial) pelos polinômios

$$
\begin{gathered}
a_{1}\left[a_{2}, a_{3}, a_{4}\right],\left[a_{1}, a_{2}\right], a_{i} \in M, \\
x_{i_{1}}^{p r_{1}} \ldots x_{i_{k}}^{p r_{k}} x_{j_{1}}^{p-1}\left[x_{j_{1}}, x_{j_{2}}\right] x_{j_{2}}^{p-1} \ldots x_{j_{2 l-1}}^{p-1}\left[x_{j_{2 l-1}}, x_{\left.j_{2 l}\right]}\right] x_{j_{2 l}}^{p-1}, \\
k \geq 0, l \geq 0, r_{s}>0, i_{1}<\ldots<i_{k}, j_{1}<\ldots<j_{2 l} .
\end{gathered}
$$

Na verdade as demonstrações do Teorema 0.1 apresentada em [7] e do Teorema 1.23 apresentada em [10] funcionam para a álgebra $Q_{3}=F\langle X\rangle / T^{(3)}$ onde $F$ é um corpo qualquer de característica $p \geq 2$. Assim podemos reescrever esses dois teoremas nas formas seguintes:

Teorema 1.24 (veja $[7,10]$ ). Seja $F$ um corpo qualquer de característica $p \geq 2$. Então $C\left(Q_{3}\right)$, o espaço vetorial dos polinômios centrais da álgebra $Q_{3}=F\langle X\rangle / T^{(3)}$, é gerado (como espaço vetorial) pelos polinômios

$$
\begin{gathered}
a_{1}\left[a_{2}, a_{3}, a_{4}\right],\left[a_{1}, a_{2}\right], a_{i} \in M, \\
x_{i_{1}}^{p r_{1}} \ldots x_{i_{k}}^{p r_{k}} x_{j_{1}}^{p-1}\left[x_{j_{1}}, x_{j_{2}}\right] x_{j_{2}}^{p-1} \ldots x_{j_{2 l-1}}^{p-1}\left[x_{j_{2 l-1}}, x_{j_{2 l} l}\right] x_{j_{2 l}}^{p-1}, \\
k \geq 0, l \geq 0, r_{s}>0, i_{1}<\ldots<i_{k}, j_{1}<\ldots<j_{2 l} .
\end{gathered}
$$

Corolário 1.25 (veja [7]). Seja $F$ um corpo qualquer de característica $p \geq 2$. Então $C\left(Q_{3}\right)$, o espaço vetorial dos polinômios centrais da álgebra $Q_{3}=F\langle X\rangle / T^{(3)}$, é gerado como $T$-subespaço de $F\langle X\rangle$ pelos polinômios

$$
x_{1}\left[x_{2}, x_{3}, x_{4}\right], x_{0}^{p}, x_{0}^{p} q_{1}, x_{0}^{p} q_{2}, \ldots, x_{0}^{p} q_{n}, \ldots
$$

Para tornar esta tese mais auto-suficiente, vamos demonstrar o Teorema 1.24 e o Corolário 1.25 .

Lema 1.26 ([7], Lema 10). Seja $F$ um corpo qualquer e $g=g\left(x_{2}, \ldots, x_{n}\right) \in F\langle X\rangle$ um polinômio que não depende de $x_{1}$. Se $x_{1} g+T^{(3)}$ for central em $Q_{3}=F\langle X\rangle / T^{(3)}$, então $g \in T^{(3)}$.

Demonstração. Usando a igualdade $[a, b c]=b[a, c]+[a, b] c$, temos $\left[x_{0}, x_{1} g\right]+T^{(3)}=$ $x_{1}\left[x_{0}, g\right]+\left[x_{0}, x_{1}\right] g+T^{(3)}$. Como $x_{1} g+T^{(3)}$ é central em $Q_{3}$, temos $\left[x_{0}, x_{1} g\right] \in T^{(3)}$. 
Assim

$$
x_{1}\left[x_{0}, g\right]+T^{(3)}=-\left[x_{0}, x_{1}\right] g+T^{(3)} .
$$

Seja $g+T^{(3)}=\sum_{t} \alpha_{t} a_{t}+T^{(3)}$ onde $\alpha_{t} \in F$ e os $a_{t}+T^{(3)}$ são elementos distintos da forma (1-17). Observe que os polinômios $a_{t}$ não dependem de $x_{0}$ e $x_{1}$ (porque $g=g\left(x_{2}, \ldots, x_{n}\right)$ não depende). Agora, aplicando a igualdade $\left[a, a_{1} \ldots a_{n}\right]=$ $\sum_{i=1}^{n} a_{1} \ldots a_{i-1}\left[a, a_{i}\right] a_{i+1} \ldots a_{n}$ (Lema $\left.1.17(\mathrm{ii})\right)$ em $\left[x_{0}, a_{t}\right]$ e observando que $\left[x_{1}, x_{2}\right]+$ $T^{(3)}$ é central em $Q_{3}$, segue que para cada $t$,

$$
\left[x_{0}, a_{t}\right]+T^{(3)}=\sum_{k} \beta_{k}^{(t)} b_{k}^{(t)}+T^{(3)}
$$

onde $\beta_{k}^{(t)} \in F$ e $b_{k}^{(t)}+T^{(3)}$ são elementos da forma (1-17) que não dependem de $x_{1} \mathrm{e}$ tais que $j_{1}=0$. Assim

$$
x_{1}\left[x_{0}, g\right]+T^{(3)}=\sum_{t} \sum_{k} \alpha_{t} \beta_{k}^{(t)} x_{1} b_{k}^{(t)}+T^{(3)}
$$

Observe que cada $x_{1} b_{k}^{(t)}$ é um polinômio de (1-17) no qual $x_{1}$ aparece na "parte não-comutador" $x_{i_{1}}^{r_{1}} \ldots x_{i_{k}}^{r_{k}}$ e a "parte comutador" $\left[x_{j_{1}}, x_{j_{2}}\right] \ldots\left[x_{j_{2 l-1}}, x_{j_{2 l}}\right]$ não depende de $x_{1}$.

Por outro lado, $\left[x_{0}, x_{1}\right] g+T^{(3)}=\sum_{t} \alpha_{t}\left[x_{0}, x_{1}\right] a_{t}+T^{(3)}$. Como os polinômios $a_{t}$ não dependem de $x_{0}$ e $x_{1}$, e $\left[x_{0}, x_{1}\right]+T^{(3)}$ é central em $Q_{3}$, os produtos $\left[x_{0}, x_{1}\right] b_{t}$ são polinômios distintos da forma (1-17) tais que $x_{1}$ aparece na "parte comutador" e a "parte não-comutador" não depende de $x_{1}$.

Mostramos assim, que existem dois conjuntos $B_{1}$ e $B_{2}$ de polinômios da forma (1-17) com $B_{1} \cap B_{2}=\varnothing$ e tais que

$$
x_{1}\left[x_{0}, g\right] \text { e }\left[x_{0}, x_{1}\right] g
$$

são, módulo $T^{(3)}$, combinações lineares de $B_{1}$ e $B_{2}$, respectivamente. Comos os elementos de (1-17) são linearmente independentes (Proposição 1.21), segue de (127) que

$$
x_{1}\left[x_{0}, g\right]+T^{(3)}=-\left[x_{0}, x_{1}\right] g+T^{(3)}=T^{(3)} .
$$

Consequentemente $\sum_{t} \alpha_{t}\left[x_{0}, x_{1}\right] a_{t}+T^{(3)}=\left[x_{0}, x_{1}\right] g+T^{(3)}=T^{(3)}$, e assim $\alpha_{t}=0$ para cada $t$. Portanto $g+T^{(3)}=\sum_{t} \alpha_{t} a_{t}+T^{(3)}=T^{(3)}$, isto é $g \in T^{(3)}$ e a demonstração está completa.

Lema 1.27 ([7], Lema 11). Seja $F$ um corpo qualquer e $f=f\left(x_{1}, \ldots, x_{n}\right) \in F\langle X\rangle$ um polinômio homogêneo de grau 1 em $x_{1}$. Se $f+T^{(3)}$ for central em $Q_{3}=F\langle X\rangle / T^{(3)}$ então $f \in\left\langle\left[x_{1}, x_{2}\right]\right\rangle^{T S}+T^{(3)}$. 
Demonstração. Seja $f=\sum_{i} \alpha_{i} a_{i} x_{1} b_{i}$ onde $\alpha_{i} \in F$ e $a_{i}, b_{i}$ são monômios (algum dos quais pode ser 1$)$. Como $a_{i} x_{1} b_{i}=x_{1} b_{i} a_{i}+\left[a_{i}, x_{1} b_{i}\right]$, temos

$$
f=x_{1} g\left(x_{2}, \ldots, x_{n}\right)+h\left(x_{1}, \ldots, x_{n}\right)
$$

onde $h\left(x_{1}, \ldots, x_{n}\right)=\sum_{i} \alpha_{i}\left[a_{i}, x_{1} b_{i}\right]$ pertence ao $T$-espaço gerado por $\left[x_{1}, x_{2}\right]$ e $g=$ $g\left(x_{2}, \ldots, x_{n}\right)$ não depende de $x_{1}$. Como $f+T^{(3)}$ e $h+T^{(3)}$ são centrais em $Q_{3}$, segue de (1-28) que $x_{1} g+T^{(3)}$ também é central em $Q_{3}$. Pelo Lema 1.26, obtemos $g \in T^{(3)}$ e novamente por (1-28) temos $f \in\left\langle\left[x_{1}, x_{2}\right]\right\rangle^{T S}+T^{(3)}$.

Proposição 1.28 (veja $[3,7,23]$ ). Seja $F$ um corpo de característica 0 . Então $C\left(Q_{3}\right)$, o espaço vetorial dos polinômios centrais da álgebra $Q_{3}=F\langle X\rangle / T^{(3)}$, é gerado como $T$-subespaço de $F\langle X\rangle$ por 1 e pelos polinômios $\left[x_{1}, x_{2}\right]$ e $x_{1}\left[x_{2}, x_{3}, x_{4}\right]$.

Demonstração. Seja $f=f\left(x_{1}, \ldots, x_{n}\right)$ um polinômio central de $Q_{3}$. Pela Proposição 1.15, $C\left(Q_{3}\right)$ é gerado como $T$-subespaço por seus polinômios multilineares. Assim podemos assumir que $f$ é multilinear. Em particular, $f$ é homogêneo de grau $1 \mathrm{em}$ $x_{1}$, logo o Lema 1.27 garante que $f$ pertence a $\left\langle\left[x_{1}, x_{2}\right]\right\rangle^{T S}+T^{(3)}$, isto é, $f$ pertence ao $T$-subespaço gerado pelos polinômios $\left[x_{1}, x_{2}\right]$ e $x_{1}\left[x_{2}, x_{3}, x_{4}\right]$.

Lema 1.29 ([7], Lema 12). Seja $F$ um corpo de característica p e $f=f\left(x_{1}, \ldots, x_{n}\right) \in$ $F\langle X\rangle$ um polinômio homogêneo de grau $m_{1}$ em $x_{1}$ onde $m_{1}$ não é um múltiplo de $p$. Se $f+T^{(3)}$ for central em $Q_{3}=F\langle X\rangle / T^{(3)}$ então $f \in\left\langle\left[x_{1}, x_{2}\right]\right\rangle^{T S}+T^{(3)}$.

Demonstração. Pela Proposição 1.16 não há perda de generalidade em supor que $f$ é multi-homogêneo de grau $m_{1}$ em $x_{1}$. Escreva $m_{1}=p q+r$ com $0<r<p$. Usando a base de $Q_{3}$ dada na Proposição 1.21, vemos que existe $g=g\left(x_{1}, \ldots, x_{n}\right) \in F\langle X\rangle$, multi-homogêneo de grau $r$ em $x_{1}$ tal que

$$
f+T^{(3)}=x_{1}^{p q} g+T^{(3)}
$$

Defina o endomorfismo $\varphi$ de $F\langle X\rangle$ por $\varphi\left(x_{1}\right)=1+x_{1}$ e $\varphi\left(x_{i}\right)=x_{i}$ se $i>1$. Por (1-29) temos

$$
\varphi(f)+T^{(3)}=\left(1+x_{1}^{p}\right)^{q} g\left(1+x_{1}, x_{2}, \ldots, x_{n}\right)+T^{(3)} .
$$

Como $g\left(x_{1}, \ldots, x_{n}\right)$ é a componente multi-homogênea (de grau $r$ em $x_{1}$ ) do polinômio $\left(1+x_{1}^{p}\right)^{q} g\left(1+x_{1}, x_{2}, \ldots, x_{n}\right)$, segue de (1-30) que $g+T^{(3)}$ é central em $Q_{3}$. Seja $h=h\left(y_{1}, \ldots, y_{r}, x_{2}, \ldots, x_{n}\right)$ a linearização total de $g$ em $x_{1}$. Então $h+T^{(3)}$ é central em $Q_{3}$ e segue do Lema 1.27 que $h \in\left\langle\left[x_{1}, x_{2}\right]\right\rangle^{T S}+T^{(3)}$. Como

$$
h\left(x_{1}, \ldots, x_{1}, x_{2}, \ldots, x_{n}\right)=r ! g\left(x_{1}, \ldots, x_{n}\right),
$$


obtemos $g \in\left\langle\left[x_{1}, x_{2}\right]\right\rangle^{T S}+T^{(3)}$. Como $x_{1}^{p}+T^{(3)}$ é central em $Q_{3}$ (Lema $\left.1.22(\mathrm{i})\right)$ e $C\left(Q_{3}\right)$ é uma álgebra (Proposição 1.13) segue que $x_{1}^{p q}+T^{(3)}$ é central em $Q_{3}$. Assim

$$
x_{1}^{p q}\left[x_{2}, x_{3}\right]+T^{(3)}=\left[x_{1}^{p q} x_{2}, x_{3}\right]+T^{(3)} .
$$

Como $g \in\left\langle\left[x_{1}, x_{2}\right]\right\rangle^{T S}+T^{(3)}$ segue de (1-29) e (1-31) que $f \in\left\langle\left[x_{1}, x_{2}\right]\right\rangle^{T S}+T^{(3)}$.

Lema 1.30 (veja $[3,7,23]$ ). Seja $F$ um corpo de característica $p \geq 2$. Então $x_{1}^{p-1}\left[x_{1}, x_{2}\right] x_{2}^{p-1}+T^{(3)}$ é central em $Q_{3}=F\langle X\rangle / T^{(3)}$.

Demonstração. Aplicando a igualdade $\left[a_{1} a_{2} \ldots a_{n}, a\right]=\sum_{i=1}^{n} a_{1} \ldots a_{i-1}\left[a_{i}, a\right] a_{i+1} \ldots a_{n}$ (Lema 1.17) temos

$$
\begin{aligned}
{\left[x_{1}^{p-1}\left[x_{1}, x_{2}\right] x_{2}^{p-1}, x_{3}\right] } & =\left[x_{1}, x_{3}\right] x_{1}^{p-2}\left[x_{1}, x_{2}\right] x_{2}^{p-1}+\ldots+x_{1}^{p-1}\left[x_{1}, x_{2}, x_{3}\right] x_{2}^{p-1}+\ldots+ \\
& +x_{1}^{p-1}\left[x_{1}, x_{2}\right] x_{2}^{p-2}\left[x_{2}, x_{3}\right]
\end{aligned}
$$

Como $\left[a_{1}, a_{2}\right]+T^{(3)}$ é central em $Q_{3}$ e $\left[a_{1}, a_{2}\right]\left[a_{1}, a_{3}\right] \in T^{(3)}$ (Lema 1.18 (ii)), obtemos

$$
\begin{aligned}
{\left[x_{1}^{p-1}\left[x_{1}, x_{2}\right] x_{2}^{p-1}, x_{3}\right] } & =\left[x_{1}, x_{3}\right]\left[x_{1}, x_{2}\right] x_{1}^{p-2} x_{2}^{p-1}+\ldots+x_{1}^{p-1}\left[x_{1}, x_{2}, x_{3}\right] x_{2}^{p-1}+\ldots+ \\
& +x_{1}^{p-1}\left[x_{1}, x_{2}\right]\left[x_{2}, x_{3}\right] x_{2}^{p-2} \equiv 0 \quad\left(\bmod T^{(3)}\right)
\end{aligned}
$$

Assim $x_{1}^{p-1}\left[x_{1}, x_{2}\right] x_{2}^{p-1}+T^{(3)}$ é central em $Q_{3}$.

\section{Demonstração do Teorema 1.24.}

Vamos verificar primeiramente que (1-24)-(1-25) são polinômios centrais da álgebra $Q_{3}=F\langle X\rangle / T^{(3)}$. É claro que $x_{1}\left[x_{2}, x_{3}, x_{4}\right]+T^{(3)}$ e $\left[x_{1}, x_{2}\right]+T^{(3)}$ são centrais em $Q_{3}$. Como $C\left(Q_{3}\right)$ é um $T$-subespaço (Proposição 1.13), segue que ele contém os polinômios da forma (1-24). Como $x_{0}^{p}+T^{(3)}$ e $x_{1}^{p-1}\left[x_{1}, x_{2}\right] x_{2}^{p-1}+T^{(3)}$ são centrais em $Q_{3}$ (Lema 1.22 (i) e Lema 1.30, respectivamente) e $C\left(Q_{3}\right)$ é uma $T$-subálgebra (Proposição 1.13), é fácil ver que os polinômios da forma (1-25) também pertencem a $C\left(Q_{3}\right)$.

Agora vamos mostrar que os polinômios da forma (1-24) e (1-25) geram $C\left(Q_{3}\right)$ como espaço vetorial. Seja $f=f\left(x_{1}, \ldots, x_{n}\right)$ um polinômio central da álgebra $Q_{3}$. Se $f \in T^{(3)}$, então $f$ é uma combinação linear de polinômios da forma $a_{1}\left[a_{2}, a_{3}, a_{4}\right]$ $\left(a_{i} \in M\right)$, que estão entre aqueles de (1-24).

Podemos então assumir que $f \notin T^{(3)}$. Pela Proposição 1.16 podemos assumir também que $f$ é multi-homogêneo de grau $m_{i}$ em cada variável $x_{i}(i=1, \ldots, n)$. Suponha que o grau de alguma variável $x_{i}$ não seja divisível por $p$. Reenumerando as variáveis $x_{i}$, podemos assumir sem perda de generalidade que $i=1$. Assim, pelo Lema 1.29 temos

$$
f \in\left\langle\left[x_{1}, x_{2}\right]\right\rangle^{T S}+T^{(3)} .
$$


Como $\left\langle\left[x_{1}, x_{2}\right]\right\rangle^{T S}$ é gerado como espaço vetorial pelos polinômios $\left[a_{1}, a_{2}\right]\left(a_{i} \in M\right)$ e $T^{(3)}$ é gerado como espaço vetorial pelos polinômios $a_{1}\left[a_{2}, a_{3}, a_{4}\right]\left(a_{i} \in M\right)$, segue de (1-32) que $f$ é uma combinação linear de polinômios da forma (1-24).

Agora suponha que todos os graus das variáveis $x_{i}$ sejam divisíveis por $p$. Já vimos que $\left[x_{1}, x_{2}\right]+T^{(3)}$ e $x^{p}+T^{(3)}$ são centrais em $Q_{3}$. Assim, segue da Proposição 1.21 que $f+T^{(3)}$ pode ser escrito como uma combinação linear de elementos da forma

$$
x_{i_{1}}^{p r_{1}} \ldots x_{i_{k}}^{p r_{k}} x_{j_{1}}^{p-1}\left[x_{j_{1}}, x_{j_{2}}\right] x_{j_{2}}^{p-1} \ldots x_{j_{2 l-1}}^{p-1}\left[x_{j_{2 l-1}}, x_{j_{2 l}}\right] x_{j_{2 l}}^{p-1}+T^{(3)}
$$

onde $k \geq 0, l \geq 0, r_{s}>0,1 \leq i_{1}<\ldots<i_{k} \leq n$ e $1 \leq j_{1}<\ldots<j_{2 l} \leq n$. Segue assim que $f$ pode ser escrito como uma combinação linear de polinômios da forma $a_{1}\left[a_{2}, a_{3}, a_{4}\right]$ $\left(a_{i} \in M\right)$ juntamente com aqueles da forma (1-25).

O lema seguinte é bem conhecido e a demonstração que daremos é apenas um esboço.

Lema 1.31. Seja $F$ um corpo de característica $p>0$. Então o polinômio $\left[x_{1}, x_{2}\right] \ldots\left[x_{2 n-1}, x_{2 n}\right]$ pertence $a\left\langle q_{n}\right\rangle^{T S}$.

Demonstração. Seja $V=\left\langle x_{1}^{p-1}\left[x_{1}, x_{2}\right] x_{2}^{p-1}\right\rangle^{T S}$. Defina o endomorfismo $\varphi$ de $F\langle X\rangle$ por $\varphi: x_{i} \rightarrow 1+\alpha x_{i}, i=1,2, \alpha \in \mathbb{Z}_{p}$. Então

$$
\sum_{k=0}^{p-1} \alpha^{k} g_{k} \in V
$$

onde $g_{k}=\left(\begin{array}{c}p-1 \\ k\end{array}\right) x_{1}^{k}\left[x_{1}, x_{2}\right] x_{2}^{p-1}$. Fazendo uso do bem conhecido argumento da matriz de Vandermonde (veja por exemplo [[13],Proposição 4.2.3]), pode ser mostrado que $g_{k} \in$ $V$ para cada $k=0, \ldots, p-2$. Em particular $g_{0}=\left[x_{1}, x_{2}\right] x_{2}^{p-1} \in V$. Usando novamente esse argumento, mas agora com $V=\left\langle\left[x_{1}, x_{2}\right] x_{2}^{p-1}\right\rangle^{T S}$, podemos mostrar que $\left[x_{1}, x_{2}\right]\langle$ pertence a $\left.\left[x_{1}, x_{2}\right] x_{2}^{p-1}\right\rangle^{T S}$. Consequentemente $\left[x_{1}, x_{2}\right]$ pertence a $\left\langle x_{1}^{p-1}\left[x_{1}, x_{2}\right] x_{2}^{p-1}\right\rangle^{T S}$. Para o caso geral, se fizermos $V=\left\langle q_{n}\right\rangle^{T S}$ e aplicarmos o mesmo argumento acima $n$ vezes, podemos mostrar que $\left[x_{1}, x_{2}\right] \ldots\left[x_{2 n-1}, x_{2 n}\right]$ pertence a $\left\langle q_{n}\right\rangle^{T S}$.

\section{Demonstração do Corolário 1.25.}

Seja $Q$ o $T$-subespaço gerado pelos polinômios (1-26). Devemos mostrar que $C\left(Q_{3}\right)=Q$. Como os polinômios (1-26) estão entre os polinômios (1-24)-(1-25) (que pertencem a $C\left(Q_{3}\right)$ pelo Teorema 1.24), temos $Q \subseteq C\left(Q_{3}\right)$.

Vamos mostrar que $C\left(Q_{3}\right) \subseteq Q$. Pelo Teorema 1.24 é suficiente mostrar que os polinômios (1-24)-(1-25) pertencem a $Q$. Como $\left[x_{1}, x_{2}\right]$ pertence ao $T$-subespaço gerado pelo polinômio $q\left(x_{1}, x_{2}\right)=x_{1}\left[x_{1}, x_{2}\right] x_{2}$ (Lema 1.31), segue que $\left[x_{1}, x_{2}\right]$ pertence 
a $Q$. Como $x_{1}\left[x_{2}, x_{3}, x_{4}\right]$ também pertence a $Q$, segue que os polinômios (1-24) pertencem a $Q$. Dividiremos o argumento restante em dois casos.

Caso $p>2$. Pelo Lema 1.22 (ii) temos

$$
\left(x_{1} x_{2}\right)^{p} \equiv x_{1}^{p} x_{2}^{p} \quad\left(\bmod T^{(3)}\right)
$$

Usando essa relação, não é difícil ver que cada polinômio da forma (1-25) pertence ao $T$-subespaço gerado pelos polinômios $x_{1}\left[x_{2}, x_{3}, x_{4}\right], x_{0}^{p}$ e $x_{0}^{p} q_{m}$ para algum $m \in \mathbb{N}$. Assim os polinômios (1-25) pertencem a $Q$ quando $\operatorname{char}(F)>2$.

Caso $p=2$. Observe que

$$
\begin{aligned}
\left(x_{1} x_{2}\right)^{2} & =x_{1} x_{2} x_{1} x_{2}=x_{1}\left(x_{1} x_{2}+\left[x_{2}, x_{1}\right]\right) x_{2}= \\
& =x_{1}^{2} x_{2}^{2}+x_{1}\left[x_{2}, x_{1}\right] x_{2}=x_{1}^{2} x_{2}^{2}+x_{1}\left[x_{1}, x_{2}\right] x_{2}
\end{aligned}
$$

Usando (1-34) não é difícil ver que cada polinômio da forma (1-25) pertence ao $T$ subespaço gerado pelos polinômios $x_{0}^{2}$ e $x_{0}^{2} q_{m}$, para algum $m \in \mathbb{N}$. Assim os polinômios (1-25) pertencem a $Q$ quando $\operatorname{char}(F)=2$.

Mostramos assim que $C\left(Q_{3}\right)=Q$, ou seja $C\left(Q_{3}\right)$ é gerado como $T$-subespaço pelos polinômios (1-26).

\subsection{Relações na álgebra $Q_{4}$}

Nesta seção exibiremos algumas relações na álgebra $F\langle X\rangle / T^{(4)}$. O Lema seguinte é bem conhecido, veja [9, 11, 16, 21, 33, 34, 44].

Lema 1.32. Seja F um corpo qualquer. Então $T^{(4)}$ contém os seguintes polinômios:

$$
\begin{gathered}
{\left[x_{1}, x_{2}, x_{3}, x_{4}\right],} \\
{\left[x_{1}, x_{2}, x_{3}\right]\left[x_{4}, x_{5}, x_{6}\right],} \\
{\left[x_{1}, x_{2}, x_{3}\right]\left[x_{4}, x_{5}\right]+\left[x_{1}, x_{2}, x_{4}\right]\left[x_{3}, x_{5}\right],\left[x_{1}, x_{2}, x_{3}\right]\left[x_{4}, x_{5}\right]+\left[x_{1}, x_{4}, x_{3}\right]\left[x_{2}, x_{5}\right],} \\
\left(\left[x_{1}, x_{2}\right]\left[x_{3}, x_{4}\right]+\left[x_{1}, x_{3}\right]\left[x_{2}, x_{4}\right]\right)\left[x_{5}, x_{6}\right] .
\end{gathered}
$$

Demonstração. É claro que o polinômio (1-35) pertence a $T^{(4)}$. Vamos mostrar que os polinômios de (1-37) pertencem a $T^{(4)}$. Usando a igualdade $[a b, c]=a[b, c]+[a, c] b$ 
obtemos

$$
\begin{aligned}
{\left[x_{1}, x_{2}, x_{3} x_{4}, x_{5}\right] } & =\left[x_{3}\left[x_{1}, x_{2}, x_{4}\right], x_{5}\right]+\left[\left[x_{1}, x_{2}, x_{3}\right] x_{4}, x_{5}\right] \\
& =x_{3}\left[x_{1}, x_{2}, x_{4}, x_{5}\right]+\left[x_{3}, x_{5}\right]\left[x_{1}, x_{2}, x_{4}\right]+ \\
& +\left[x_{1}, x_{2}, x_{3}\right]\left[x_{4}, x_{5}\right]+\left[x_{1}, x_{2}, x_{3}, x_{5}\right] x_{4}
\end{aligned}
$$

Como os polinômios $\left[x_{1}, x_{2}, x_{3} x_{4}, x_{5}\right], x_{3}\left[x_{1}, x_{2}, x_{4}, x_{5}\right]$ e $\left[x_{1}, x_{2}, x_{3}, x_{5}\right] x_{4}$ pertencem a $T^{(4)}$, temos

$$
\left[x_{1}, x_{2}, x_{3}\right]\left[x_{4}, x_{5}\right]+\left[x_{3}, x_{5}\right]\left[x_{1}, x_{2}, x_{4}\right] \in T^{(4)} \text {. }
$$

Como $\left[x_{3}, x_{5}\right]\left[x_{1}, x_{2}, x_{4}\right] \equiv\left[x_{1}, x_{2}, x_{4}\right]\left[x_{3}, x_{5}\right]\left(\bmod T^{(4)}\right)$, segue de $(1-39)$ que

$$
\left[x_{1}, x_{2}, x_{3}\right]\left[x_{4}, x_{5}\right]+\left[x_{1}, x_{2}, x_{4}\right]\left[x_{3}, x_{5}\right] \in T^{(4)} .
$$

Agora, pelo Lema 1.17 (i) e pela igualdade $[a b, c]=a[b, c]+[a, c] b$ temos

$$
\begin{aligned}
{\left[x_{1} x_{2}, x_{3}, x_{4}, x_{5}\right] } & =\left[x_{1}\left[x_{2}, x_{3}, x_{4}\right], x_{5}\right]+\left[\left[x_{1}, x_{3}\right]\left[x_{2}, x_{4}\right], x_{5}\right]+ \\
& +\left[\left[x_{1}, x_{4}\right]\left[x_{2}, x_{3}\right], x_{5}\right]+\left[\left[x_{1}, x_{3}, x_{4}\right] x_{2}, x_{5}\right] \\
& =x_{1}\left[x_{2}, x_{3}, x_{4}, x_{5}\right]+\left[x_{1}, x_{5}\right]\left[x_{2}, x_{3}, x_{4}\right]+\left[x_{1}, x_{3}\right]\left[x_{2}, x_{4}, x_{5}\right]+ \\
& +\left[x_{1}, x_{3}, x_{5}\right]\left[x_{2}, x_{4}\right]+\left[x_{1}, x_{4}\right]\left[x_{2}, x_{3}, x_{5}\right]+\left[x_{1}, x_{4}, x_{5}\right]\left[x_{2}, x_{3}\right]+ \\
& +\left[x_{1}, x_{3}, x_{4}\right]\left[x_{2}, x_{5}\right]+\left[x_{1}, x_{3}, x_{4}, x_{5}\right] x_{2} .
\end{aligned}
$$

Observe que os polinômios $\left[x_{1} x_{2}, x_{3}, x_{4}, x_{5}\right], x_{1}\left[x_{2}, x_{3}, x_{4}, x_{5}\right]$ e $\left[x_{1}, x_{3}, x_{4}, x_{5}\right] x_{2}$ pertencem a $T^{(4)}$. Por (1-40), o polinômio $\left[x_{1}, x_{3}, x_{5}\right]\left[x_{2}, x_{4}\right]+\left[x_{1}, x_{3}, x_{4}\right]\left[x_{2}, x_{5}\right]$ também pertence a $T^{(4)}$. Assim, segue de (1-41) que

$$
\left[x_{1}, x_{5}\right]\left[x_{2}, x_{3}, x_{4}\right]+\left[x_{1}, x_{3}\right]\left[x_{2}, x_{4}, x_{5}\right]+\left[x_{1}, x_{4}\right]\left[x_{2}, x_{3}, x_{5}\right]+\left[x_{1}, x_{4}, x_{5}\right]\left[x_{2}, x_{3}\right] \in T^{(4)}
$$

Como $\left[a_{1}, a_{2}\right]\left[a_{3}, a_{4}, a_{5}\right] \equiv\left[a_{3}, a_{4}, a_{5}\right]\left[a_{1}, a_{2}\right]\left(\bmod T^{(4)}\right)$, segue de $(1-42)$ que

$$
\left[x_{2}, x_{3}, x_{4}\right]\left[x_{1}, x_{5}\right]+\left[x_{2}, x_{4}, x_{5}\right]\left[x_{1}, x_{3}\right]+\left[x_{2}, x_{3}, x_{5}\right]\left[x_{1}, x_{4}\right]+\left[x_{1}, x_{4}, x_{5}\right]\left[x_{2}, x_{3}\right] \in T^{(4)} \text {. }
$$

Novamente por (1-40), o polinômio $\left[x_{2}, x_{3}, x_{4}\right]\left[x_{1}, x_{5}\right]+\left[x_{2}, x_{3}, x_{5}\right]\left[x_{1}, x_{4}\right]$ pertence a $T^{(4)}$. Logo (1-43) implica que

$$
\left[x_{2}, x_{4}, x_{5}\right]\left[x_{1}, x_{3}\right]+\left[x_{1}, x_{4}, x_{5}\right]\left[x_{2}, x_{3}\right] \in T^{(4)},
$$

e consequentemente

$$
\left[x_{1}, x_{2}, x_{3}\right]\left[x_{4}, x_{5}\right]+\left[x_{1}, x_{4}, x_{3}\right]\left[x_{2}, x_{5}\right] \in T^{(4)} \text {. }
$$


Por (1-40) e (1-44), vemos que os polinômios de (1-37) pertencem a $T^{(4)}$. Vamos mostrar que o polinômio (1-36) pertence a $T^{(4)}$. Por (1-40) temos

$$
\left[x_{1}, x_{2}, x_{3}\right]\left[\left[x_{4}, x_{5}\right], x_{6}\right]+\left[x_{1}, x_{2},\left[x_{4}, x_{5}\right]\right]\left[x_{3}, x_{6}\right] \in T^{(4)} \text {. }
$$

Como $\left[x_{1}, x_{2},\left[x_{4}, x_{5}\right]\right]=\left[x_{1}, x_{2}, x_{4}, x_{5}\right]-\left[x_{1}, x_{2}, x_{5}, x_{4}\right] \in T^{(4)}$, obtemos

$$
\left[x_{1}, x_{2}, x_{3}\right]\left[x_{4}, x_{5}, x_{6}\right] \in T^{(4)}
$$

Finalmente, vamos mostrar que o polinômio (1-38) pertence a $T^{(4)}$. Por (1-44) obtemos

$$
\left[x_{1} x_{2}, x_{3}, x_{4}\right]\left[x_{5}, x_{6}\right]+\left[x_{5}, x_{3}, x_{4}\right]\left[x_{1} x_{2}, x_{6}\right] \in T^{(4)} .
$$

Seja $\omega\left(x_{1}, x_{2}, x_{3}, x_{4}\right)=\left[x_{1}, x_{2}\right]\left[x_{3}, x_{4}\right]+\left[x_{1}, x_{3}\right]\left[x_{2}, x_{4}\right]$. Por um lado, segue do Lema 1.17 (i) que

$$
\begin{aligned}
{\left[x_{1} x_{2}, x_{3}, x_{4}\right]\left[x_{5}, x_{6}\right] } & =x_{1}\left[x_{2}, x_{3}, x_{4}\right]\left[x_{5}, x_{6}\right]-\omega\left(x_{1}, x_{3}, x_{4}, x_{2}\right)\left[x_{5}, x_{6}\right]+\left[x_{1}, x_{3}, x_{4}\right] x_{2}\left[x_{5}, x_{6}\right] \\
& =x_{1}\left[x_{2}, x_{3}, x_{4}\right]\left[x_{5}, x_{6}\right]+x_{2}\left[x_{1}, x_{3}, x_{4}\right]\left[x_{5}, x_{6}\right]+\left[x_{1}, x_{3}, x_{4}, x_{2}\right]\left[x_{5}, x_{6}\right]- \\
& -\omega\left(x_{1}, x_{3}, x_{4}, x_{2}\right)\left[x_{5}, x_{6}\right]
\end{aligned}
$$

isto é

$$
\begin{aligned}
{\left[x_{1} x_{2}, x_{3}, x_{4}\right]\left[x_{5}, x_{6}\right] } & \equiv x_{1}\left[x_{2}, x_{3}, x_{4}\right]\left[x_{5}, x_{6}\right]+x_{2}\left[x_{1}, x_{3}, x_{4}\right]\left[x_{5}, x_{6}\right]- \\
& -\omega\left(x_{1}, x_{3}, x_{4}, x_{2}\right)\left[x_{5}, x_{6}\right]\left(\bmod T^{(4)}\right) .
\end{aligned}
$$

Por outro lado

$$
\begin{aligned}
{\left[x_{5}, x_{3}, x_{4}\right]\left[x_{1} x_{2}, x_{6}\right] } & =\left[x_{5}, x_{3}, x_{4}\right] x_{1}\left[x_{2}, x_{6}\right]+\left[x_{5}, x_{3}, x_{4}\right]\left[x_{1}, x_{6}\right] x_{2} \\
& =x_{1}\left[x_{5}, x_{3}, x_{4}\right]\left[x_{2}, x_{6}\right]+\left[x_{5}, x_{3}, x_{4}, x_{1}\right]\left[x_{2}, x_{6}\right]+ \\
& +x_{2}\left[x_{5}, x_{3}, x_{4}\right]\left[x_{1}, x_{6}\right]+\left[x_{5}, x_{3}, x_{4}, x_{2}\right]\left[x_{1}, x_{6}\right]+\left[x_{5}, x_{3}, x_{4}\right]\left[x_{1}, x_{6}, x_{2}\right] .
\end{aligned}
$$

Usando (1-45), obtemos

$$
\left[x_{5}, x_{3}, x_{4}\right]\left[x_{1} x_{2}, x_{6}\right] \equiv x_{1}\left[x_{5}, x_{3}, x_{4}\right]\left[x_{2}, x_{6}\right]+x_{2}\left[x_{5}, x_{3}, x_{4}\right]\left[x_{1}, x_{6}\right] \quad\left(\bmod T^{(4)}\right) .
$$

Somando (1-47) e (1-48), segue de (1-46) que

$$
\begin{aligned}
x_{1}\left(\left[x_{2}, x_{3}, x_{4}\right]\left[x_{5}, x_{6}\right]+\left[x_{5}, x_{3}, x_{4}\right]\left[x_{2}, x_{6}\right]\right) & +x_{2}\left(\left[x_{1}, x_{3}, x_{4}\right]\left[x_{5}, x_{6}\right]+\left[x_{5}, x_{3}, x_{4}\right]\left[x_{1}, x_{6}\right]\right)- \\
& -\omega\left(x_{1}, x_{3}, x_{4}, x_{2}\right)\left[x_{5}, x_{6}\right] \in T^{(4)} .
\end{aligned}
$$


Finalmente, por (1-44), segue que $\omega\left(x_{1}, x_{3}, x_{4}, x_{2}\right)\left[x_{5}, x_{6}\right] \in T^{(4)}$ e portanto $\omega\left(x_{1}, x_{2}, x_{3}, x_{4}\right)\left[x_{5}, x_{6}\right] \in T^{(4)}$. Mostramos assim que todos os polinômios de (135)-(1-38) pertecem a $T^{(4)}$, o que conclui a demonstração do lema.

Na verdade os polinômios (1-35)-(1-36) do Lema 1.32 geram $T^{(4)}$ como ideal bilateral de $F\langle X\rangle$ (veja [[11], Teorema 1.3]), mas não precisaremos desse resultado mais geral nesta tese. O corolário seguinte é bem conhecido, veja [11, 16, 21, 33, 44].

Corolário 1.33. Seja $F$ um corpo qualquer e $a_{1}, a_{2}, \ldots, a_{2 k+1}$ polinômios de $F\langle X\rangle$. Então

(i) $\left[a_{\sigma(1)}, a_{\sigma(2)}\right] \ldots\left[a_{\sigma(2 k-1)}, a_{\sigma(2 k)}\right] \equiv(-1)^{\sigma}\left[a_{1}, a_{2}\right] \ldots\left[a_{2 k-1}, a_{2 k}\right]\left(\bmod T^{(4)}\right), k \geq$ $3, \sigma \in S_{2 k}$,

(ii) $\left[a_{\sigma(1)}, a_{\sigma(2)}, a_{\sigma(3)}\right]\left[a_{\sigma(4)}, a_{\sigma(5)}\right] \ldots\left[a_{\sigma(2 k)}, a_{\sigma(2 k+1)}\right]$ $\equiv(-1)^{\sigma}\left[a_{1}, a_{2}, a_{3}\right]\left[a_{4}, a_{5}\right] \ldots\left[a_{2 k}, a_{2 k+1}\right]\left(\bmod T^{(4)}\right), k \geq 2, \sigma \in S_{2 k+1}$,

(iii) $\left[a_{1}, a_{2}, a_{3}\right]\left[a_{4}, a_{5}\right] \in T^{(4)}$ desde que char $(F) \neq 3$.

Demonstração. Comecemos por (i). Vamos provar a afirmação para $k=3$, isto é

$$
\left[a_{\sigma(1)}, a_{\sigma(2)}\right]\left[a_{\sigma(3)}, a_{\sigma(4)}\right]\left[a_{\sigma(5)}, a_{\sigma(6)}\right] \equiv(-1)^{\sigma}\left[a_{1}, a_{2}\right]\left[a_{3}, a_{4}\right]\left[a_{5}, a_{6}\right] \quad\left(\bmod T^{(4)}\right)
$$

para qualquer $\sigma \in S_{6}$; o caso geral é análogo. É claro que (1-49) é válido para $\sigma=(12), \sigma=(34)$ e $\sigma=(56)$. Como $\left[x_{1}, x_{2}\right]\left[x_{3}, x_{4}\right]\left[x_{5}, x_{6}\right]+\left[x_{1}, x_{3}\right]\left[x_{2}, x_{4}\right]\left[x_{5}, x_{6}\right] \in$ $T^{(4)}$ (Lema 1.32) vemos que (1-49) vale para $\sigma=(23)$. Como $\left[\left[a_{1}, a_{2}\right],\left[a_{3}, a_{4}\right]\right]=$ $\left[a_{1}, a_{2}, a_{3}, a_{4}\right]-\left[a_{1}, a_{2}, a_{4}, a_{3}\right] \in T^{(4)}$, temos $\left[a_{1}, a_{2}\right]\left[a_{3}, a_{4}\right] \equiv\left[a_{3}, a_{4}\right]\left[a_{1}, a_{2}\right]\left(\bmod T^{(4)}\right)$. Usando a última relação e observando que $\left[x_{3}, x_{4}\right]\left[x_{5}, x_{6}\right]\left[x_{1}, x_{2}\right]+\left[x_{3}, x_{5}\right]\left[x_{4}, x_{6}\right]\left[x_{1}, x_{2}\right] \in$ $T^{(4)}$ (Lema 1.32), obtemos

$$
\begin{aligned}
& {\left[x_{1}, x_{2}\right]\left[x_{3}, x_{4}\right]\left[x_{5}, x_{6}\right] \equiv\left[x_{3}, x_{4}\right]\left[x_{5}, x_{6}\right]\left[x_{1}, x_{2}\right] \quad\left(\bmod T^{(4)}\right)} \\
& \equiv-\left[x_{3}, x_{5}\right]\left[x_{4}, x_{6}\right]\left[x_{1}, x_{2}\right] \quad\left(\bmod T^{(4)}\right) \\
& \equiv-\left[x_{1}, x_{2}\right]\left[x_{3}, x_{5}\right]\left[x_{4}, x_{6}\right] \quad\left(\bmod T^{(4)}\right) \text {. }
\end{aligned}
$$

Portanto (1-49) é válido para $\sigma=(45)$. Como $S_{6}$ é gerado pelas permutações (12), (23), (34), (45) e (56), segue que (1-49) é válido para qualquer $\sigma \in S_{6}$.

Agora vamos provar (ii). Consideraremos apenas o caso $k=5$, isto é

$$
\left[x_{\sigma(1)}, x_{\sigma(2)}, x_{\sigma(3)}\right]\left[x_{\sigma(4)}, x_{\sigma(5)}\right] \equiv(-1)^{\sigma}\left[x_{1}, x_{2}, x_{3}\right]\left[x_{4}, x_{5}\right] \quad\left(\bmod T^{(4)}\right)
$$

para qualquer $\sigma \in S_{5}$; o caso geral é análogo. É claro que (1-50) é válido para $\sigma=(12)$ e $\sigma=(45)$. Vimos no Lema 1.32 que os polinômios $\left[x_{1}, x_{2}, x_{3}\right]\left[x_{4}, x_{5}\right]+\left[x_{1}, x_{2}, x_{4}\right]\left[x_{3}, x_{5}\right]$ 
e $\left[x_{1}, x_{2}, x_{3}\right]\left[x_{4}, x_{5}\right]+\left[x_{1}, x_{4}, x_{3}\right]\left[x_{2}, x_{5}\right]$ pertencem a $T^{(4)}$. Isso mostra que (1-50) também é válido para $\sigma=(34)$ e $\sigma=(24)$. Como $S_{5}$ é gerado pelas permutações (12), (24), (34) e (45), segue que (1-50) é válido para qualquer $\sigma \in S_{5}$.

Vejamos (iii). A identidade de Jacobi nos fornece

$$
\left[a_{1}, a_{2}, a_{3}\right]\left[a_{4}, a_{5}\right]+\left[a_{2}, a_{3}, a_{1}\right]\left[a_{4}, a_{5}\right]+\left[a_{3}, a_{1}, a_{2}\right]\left[a_{4}, a_{5}\right]=0
$$

Por (1-50) isso implica que $3\left[a_{1}, a_{2}, a_{3}\right]\left[a_{4}, a_{5}\right] \in T^{(4)}$. Como $\operatorname{char}(F) \neq 3$, obtemos $\left[a_{1}, a_{2}, a_{3}\right]\left[a_{4}, a_{5}\right] \in T^{(4)}$. 


\section{Polinômios centrais da álgebra $Q_{4}$}

Neste capítulo daremos uma descrição dos polinômios centrais da álgebra $Q_{4}$ onde $F$ é um corpo qualquer. Dividimos o capítulo em duas seções: na primeira tratamos o caso de característica $p \neq 3$ e na segunda o caso de característica $p=3$.

\section{$2.1 \quad \mathrm{O}$ caso de característica $\neq 3$}

O objetivo desta seção é fornecer uma descrição dos polinômios centrais da álgebra $Q_{4}$ onde $F$ é um corpo de característica $p \neq 3$. Mais precisamente, provaremos o segundo e o terceiro resultado principal desta tese.

Teorema 2.1. Seja $F$ um corpo de característica 2. Então $C\left(Q_{4}\right)$, o espaço vetorial dos polinômios centrais da álgebra $Q_{4}=F\langle X\rangle / T^{(4)}$, é gerado como $T$-subespaço de $F\langle X\rangle$ pelos polinômios

$$
x_{1}\left[x_{2}, x_{3}, x_{4}\right], x_{0}^{4}, x_{0}^{2} q_{2}, x_{0}^{2} q_{3}, x_{0}^{2} q_{4}, \ldots, x_{0}^{2} q_{n}, \ldots
$$

Teorema 2.2. Seja $F$ um corpo de característica $p>3$. Então $C\left(Q_{4}\right)$, o espaço vetorial dos polinômios centrais de $Q_{4}=F\langle X\rangle / T^{(4)}$ é gerado (como espaço vetorial) pelos polinômios

$$
\begin{aligned}
& {\left[a_{1}, a_{2}\right]\left[a_{3}, a_{4}\right], a_{1}\left[a_{2}, a_{3}, a_{4}\right], a_{i} \in M,} \\
& x_{i_{1}}^{p r_{1}} \ldots x_{i_{k}}^{p r_{k}} x_{j_{1}}^{p-1}\left[x_{j_{1}}, x_{j_{2}}\right] x_{j_{2}}^{p-1} \ldots x_{j_{2 l-1}}^{p-1}\left[x_{j_{2 l-1}}, x_{j_{2 l}}\right] x_{j_{2 l}}^{p-1}, \\
& k \geq 0, l=0 \text { ou } l \geq 2, r_{s}>0, i_{1}<\ldots<i_{k}, j_{1}<\ldots<j_{2 l} .
\end{aligned}
$$

Começaremos estudando a forma dos polinômios centrais de $Q_{4}$ que são homogêneos de grau 1 em $x_{1}$.

Lema 2.3. Seja $F$ um corpo qualquer e $f=f\left(x_{1}, \ldots, x_{n}\right) \in F\langle X\rangle$ um polinômio homogêneo de grau 1 em $x_{1}$ tal que $f$ pode ser escrito, módulo $T^{(3)}$, como uma 
combinação linear de polinômios da forma

$$
x_{i_{1}}^{r_{1}} \ldots x_{i_{k}}^{r_{k}}\left[x_{j_{1}}, x_{j_{2}}\right] \ldots\left[x_{j_{2 l-1}}, x_{j_{2 l}}\right]
$$

onde $k \geq 0, l \geq 2, r_{s}>0,1 \leq i_{1}<\ldots<i_{k} \leq n$ e $1 \leq j_{1}<\ldots<j_{2 l} \leq n$. Então existem polinômios $g_{1}=g_{1}\left(x_{2}, \ldots, x_{n}\right) \in F\langle X\rangle$ (que não depende de $x_{1}$ ), $g_{2} \in\left\langle\left[x_{1}, x_{2}\right]\left[x_{3}, x_{4}\right]\right\rangle^{T S}$ e $g_{3} \in T^{(3)}$ tais que $f=x_{1} g_{1}+g_{2}+g_{3}$.

Demonstração. Se mostrarmos que para cada polinômio $h$ da forma (2-3) existem polinômios $h_{1}=h_{1}\left(x_{2}, \ldots, x_{n}\right) \in F\langle X\rangle$ (que não depende de $x_{1}$ ), $h_{2} \in\left\langle\left[x_{1}, x_{2}\right]\left[x_{3}, x_{4}\right]\right\rangle^{T S}$ e $h_{3} \in T^{(3)}$ tais que $h=x_{1} h_{1}+h_{2}+h_{3}$, então é fácil ver que existem polinômios $g_{1}=g_{1}\left(x_{2}, \ldots, x_{n}\right) \in F\langle X\rangle$ (que não depende de $\left.x_{1}\right), g_{2} \in\left\langle\left[x_{1}, x_{2}\right]\left[x_{3}, x_{4}\right]\right\rangle^{T S}$ e $g_{3} \in T^{(3)}$ tais que $f=x_{1} g_{1}+g_{2}+g_{3}$.

Seja $h$ um polinômio da forma (2-3). Como $h$ é homogêneo de grau 1 em $x_{1}$, segue que $i_{1}=1$ ou $j_{1}=1$. Suponha $i_{1}=1$. Pondo $h_{1}=x_{i_{2}}^{r_{2}} \ldots x_{i_{k}}^{r_{k}}\left[x_{j_{1}}, x_{j_{2}}\right] \ldots\left[x_{j_{2 l-1}}, x_{j_{2 l}}\right]$ temos $h=x_{1} h_{1}$, que está na forma desejada pois $h_{1}$ não depende de $x_{1}$. Agora seja $j_{1}=1$. Pondo $a=x_{i_{1}}^{r_{1}} \ldots x_{i_{k}}^{r_{k}}$, sendo $l \geq 2$, podemos escrever $h=a\left[x_{1}, x_{j_{2}}\right] b\left[x_{j_{2 l-1}}, x_{j_{2 l}}\right]$ onde $b$ é 1 ou um produto de comutadores. Agora

$$
\begin{aligned}
a\left[x_{1}, x_{j_{2}}\right] b\left[x_{j_{2 l-1}}, x_{j_{2 l}}\right] & =\left[a x_{1}, x_{j_{2}}\right] b\left[x_{j_{2 l-1}}, x_{j_{2 l}}\right]-\left[a, x_{j_{2}}\right] x_{1} b\left[x_{j_{2 l-1}}, x_{j_{2 l}}\right] \\
& =\left[a x_{1} b, x_{j_{2}}\right]\left[x_{j_{2 l-1}}, x_{j_{2 l}}\right]-a x_{1}\left[b, x_{j_{2}}\right]\left[x_{j_{2 l-1}}, x_{j_{2 l}}\right]- \\
& -x_{1} b\left[a, x_{j_{2}}\right]\left[x_{j_{2 l-1}}, x_{j_{2 l}}\right]-\left[a, x_{j_{2}}, x_{1} b\right]\left[x_{j_{2 l-1}}, x_{j_{2 l}}\right] .
\end{aligned}
$$

Sejam $h_{1}=-b\left[a, x_{j_{2}}\right]\left[x_{j_{2 l-1}}, x_{j_{2 l} l}\right], h_{2}=\left[a x_{1} b, x_{j_{2}}\right]\left[x_{j_{2 l-1}}, x_{j_{2 l}}\right]$ e $h_{3}=-a x_{1}\left[b, x_{j_{2}}\right]\left[x_{j_{2 l-1}}, x_{j_{2 l}}\right]-$ $\left[a, x_{j_{2}}, x_{1} b\right]\left[x_{j_{2 l-1}}, x_{j_{2 l}}\right]$. Observe que $h_{1}$ não depende de $x_{1}$ e $h_{2} \in\left\langle\left[x_{1}, x_{2}\right]\left[x_{3}, x_{4}\right]\right\rangle^{T S}$. Observe também que $h_{3} \in T^{(3)}$ porque $\left[b, x_{j_{2}}\right] \in T^{(3)}$. Assim $h=x_{1} h_{1}+h_{2}+h_{3}$ está na forma desejada, e isso conclui a demonstração.

Lema 2.4. Seja $F$ um corpo qualquer e $f=f\left(x_{1}, \ldots, x_{n}\right) \in F\langle X\rangle$ um polinômio homogêneo de grau 1 em $x_{1}$. Se $f+T^{(4)}$ for central em $Q_{4}=F\langle X\rangle / T^{(4)}$, então $f \in\left\langle\left[x_{1}, x_{2}\right]\left[x_{3}, x_{4}\right]\right\rangle^{T S}+T^{(3)}$.

Demonstração. Pela Proposição 1.16, não há perda de generalidade em supor que $f$ é multi-homogêneo. Segue da Proposição 1.21 que $f$ pode ser escrito, módulo $T^{(3)}$, como uma combinação linear de polinômios da forma

$$
x_{i_{1}}^{r_{1}} \ldots x_{i_{k}}^{r_{k}}\left[x_{j_{1}}, x_{j_{2}}\right] \ldots\left[x_{j_{2 l-1}}, x_{j_{2 l}}\right]
$$

onde $k \geq 0, l \geq 0, r_{s}>0,1 \leq i_{1}<\ldots<i_{k} \leq n$ e $1 \leq j_{1}<\ldots<j_{2 l} \leq n$. Primeiramente, vamos mostrar que $l \geq 2$ para todos os polinômios de (2-4). 
Afirmação 1: Temos $l \neq 0$ em todos os polinômios de (2-4). De fato, seja

$$
x_{i_{1}}^{r_{1}} x_{i_{2}}^{r_{2}} \ldots x_{i_{n}}^{r_{n}}
$$

um polinômio de (2-4) com coeficiente $\alpha \in F$. Vamos mostrar que $\alpha=0$. Como cada polinômio de (2-4) é homogêneo de grau 1 em $x_{1}$, temos $i_{1}=1$ e $r_{1}=1$ no polinômio (2-5). Defina o endomorfismo $\varphi$ de $F\langle X\rangle$ por $\varphi\left(x_{1}\right)=x_{1}$ e $\varphi\left(x_{s}\right)=1$ se $s \neq 1$. Segue de (2-4) e (2-5) que $\varphi(f)+T^{(3)}=\alpha x_{1}+T^{(3)}$. Como $C\left(Q_{4}\right)+T^{(3)} \subseteq C\left(Q_{3}\right)$, temos que $\alpha x_{1}+T^{(3)}$ é central em $Q_{3}$. Mas isso implica $\alpha=0$ porque $x_{1}+T^{(3)}$ não é central em $Q_{3}$.

Afirmação 2: Temos $l \neq 1$ em todos os polinômios de (2-4). De fato, seja

$$
x_{i_{1}}^{r_{1}} \ldots x_{i_{k}}^{r_{k}}\left[x_{j_{1}}, x_{j_{2}}\right]
$$

um polinômio de (2-4) com coeficiente $\beta$. Vamos mostrar que $\beta=0$. Defina o endomorfismo $\phi$ de $F\langle X\rangle$ por $\phi\left(x_{j_{s}}\right)=x_{j_{s}}$, se $s \in\left\{j_{1}, j_{2}\right\}$ e $\phi\left(x_{s}\right)=1$, se $s \notin\left\{j_{1}, j_{2}\right\}$. Então segue de (2-4) e (2-6) que

$$
\phi(f)+T^{(3)}=\beta x_{j_{1}}^{r_{1}} x_{j_{2}}^{r_{2}}\left[x_{j_{1}}, x_{j_{2}}\right]+T^{(3)} .
$$

Seja $g\left(x_{j_{1}}, x_{j_{2}}\right)=x_{j_{1}}^{r_{1}} x_{j_{2}}^{r_{2}}\left[x_{j_{1}}, x_{j_{2}}\right]$. Observe que $\left[x_{j_{1}}, x_{j_{2}}\right]$ é uma componente multihomogênea do polinômio $g\left(x_{j_{1}}+1, x_{j_{2}}+1\right)=\left(x_{j_{1}}+1\right)^{r_{1}}\left(x_{j_{2}}+1\right)^{r_{2}}\left[x_{j_{1}}, x_{j_{2}}\right]$. Suponha que $\beta \neq 0$. Então $g\left(x_{j_{1}}+1, x_{j_{2}}+1\right)$ pertence a $C\left(Q_{4}\right)+T^{(3)}$ e como $C\left(Q_{4}\right)+T^{(3)}$ é um $T$-subespaço multi-homogêneo (Proposições 1.10 e 1.16, respectivamente), concluímos que $\left[x_{j_{1}}, x_{j_{2}}\right]$ pertence a $C\left(Q_{4}\right)+T^{(3)}$. Assim existem $h_{1} \in C\left(Q_{4}\right)$ e $h_{2} \in T^{(3)}$ tais que

$$
\left[x_{j_{1}}, x_{j_{2}}\right]=h_{1}+h_{2} \text {. }
$$

Como $h_{1} \neq 0$ (porque $\left[x_{j_{1}}, x_{j_{2}}\right]$ não pertence a $T^{(3)}$ ) e $T^{(3)}$ é multi-homogêneo, podemos supor que $h_{1}$ é multi-homogêneo de grau 1 em $x_{j_{1}}$ e $x_{j_{2}}$, e grau 0 nas demais variáveis. Portanto $h_{1}$ é um polinômio de grau 2 e $\left[h_{1}, x_{n+1}\right]$ possui grau 3 . Logo $\left[h_{1}, x_{n+1}\right]$ não pertence a $T^{(4)}$, isto é, $h_{1}$ não pertence a $C\left(Q_{4}\right)$, uma contradição. Portanto deve ser $\beta=0$. Segue das Afirmações 1 e 2 que $l \geq 2$ para todos os polinômios de (2-4).

Reunimos todas as hipóteses do Lema 2.3 e isso garante a existência de polinômios $g_{1}=g_{1}\left(x_{2}, \ldots, x_{n}\right) \in F\langle X\rangle$ (que não depende de $x_{1}$ ), $g_{2} \in\left\langle\left[x_{1}, x_{2}\right]\left[x_{3}, x_{4}\right]\right\rangle^{T S}$ e $g_{3} \in T^{(3)}$ tais que

$$
f=x_{1} g_{1}+g_{2}+g_{3} \text {. }
$$

Como $f, g_{2}$ e $g_{3}$ são polinômios centrais de $Q_{3}$, segue que $x_{1} g_{1}$ também é um 
polinômio central de $Q_{3}$. Aplicando o Lema 1.26 obtemos $g_{1} \in T^{(3)}$. Assim, por (2-7) temos que $f$ pertence a $\left\langle\left[x_{1}, x_{2}\right]\left[x_{3}, x_{4}\right]\right\rangle^{T S}+T^{(3)}$.

Lema 2.5 (veja $[22,25]$ ). Seja $F$ um corpo de característica $\neq 3$. Então $\left[x_{1}, x_{2}\right]\left[x_{3}, x_{4}\right]+T^{(4)}$ e $x_{1}\left[x_{2}, x_{3}, x_{4}\right]+T^{(4)}$ são centrais na álgebra $Q_{4}=F\langle X\rangle / T^{(4)}$.

Demonstração. Primeiramente vamos mostrar que $\left[x_{1}, x_{2}\right]\left[x_{3}, x_{4}\right]+T^{(4)}$ é central em $Q_{4}$. De fato, usando a igualdade $[a b, c]=a[b, c]+[a, c] b$, temos

$$
\begin{aligned}
{\left[\left[x_{1}, x_{2}\right]\left[x_{3}, x_{4}\right], x_{5}\right] } & =\left[x_{1}, x_{2}\right]\left[x_{3}, x_{4}, x_{5}\right]+\left[x_{1}, x_{2}, x_{5}\right]\left[x_{3}, x_{4}\right] \\
& =\left[x_{3}, x_{4}, x_{5}\right]\left[x_{1}, x_{2}\right]+\left[\left[x_{1}, x_{2}\right],\left[x_{3}, x_{4}, x_{5}\right]\right]+\left[x_{1}, x_{2}, x_{5}\right]\left[x_{3}, x_{4}\right] .
\end{aligned}
$$

Pelo Corolário 1.33 (iii), os polinômios $\left[x_{3}, x_{4}, x_{5}\right]\left[x_{1}, x_{2}\right]$ e $\left[x_{1}, x_{2}, x_{5}\right]\left[x_{3}, x_{4}\right]$ pertencem a $T^{(4)}$. Como $\left[\left[x_{1}, x_{2}\right],\left[x_{3}, x_{4}, x_{5}\right]\right]$ também pertence a $T^{(4)}$, segue que $\left[\left[x_{1}, x_{2}\right]\left[x_{3}, x_{4}\right], x_{5}\right]$ pertence a $T^{(4)}$, isto é, $\left[x_{1}, x_{2}\right]\left[x_{3}, x_{4}\right]+T^{(4)}$ é central em $Q_{4}$. Vamos mostrar agora que $x_{1}\left[x_{2}, x_{3}, x_{4}\right]+T^{(4)}$ é central em $Q_{4}$. De fato, como

$$
\left[x_{1}\left[x_{2}, x_{3}, x_{4}\right], x_{5}\right]=x_{1}\left[x_{2}, x_{3}, x_{4}, x_{5}\right]+\left[x_{1}, x_{5}\right]\left[x_{2}, x_{3}, x_{4}\right]
$$

e os polinômios $x_{1}\left[x_{2}, x_{3}, x_{4}, x_{5}\right],\left[x_{1}, x_{5}\right]\left[x_{2}, x_{3}, x_{4}\right]$ pertencem a $T^{(4)}$ segue que $\left[x_{1}\left[x_{2}, x_{3}, x_{4}\right], x_{5}\right]$ pertence a $T^{(4)}$, isto é, $x_{1}\left[x_{2}, x_{3}, x_{4}\right]+T^{(4)}$ é central em $Q_{4}$.

Proposição 2.6 (veja [25]). Seja $F$ um corpo de característica 0 . Então $C\left(Q_{4}\right)$, o espaço vetorial dos polinômios centrais da álgebra $Q_{4}=F\langle X\rangle / T^{(4)}$ é gerado, como $T$-subespaço de $F\langle X\rangle$, por 1 e pelos polinômios $\left[x_{1}, x_{2}\right]\left[x_{3}, x_{4}\right]$ e $x_{1}\left[x_{2}, x_{3}, x_{4}\right]$.

Demonstração. Já vimos no Lema 2.5 que $\left[x_{1}, x_{2}\right]\left[x_{3}, x_{4}\right]$ e $x_{1}\left[x_{2}, x_{3}, x_{4}\right]$ são polinômios centrais de $Q_{4}$. Vamos mostrar que esses polinômios geram $C\left(Q_{4}\right)$ como $T$-subespaço. Seja $f=f\left(x_{1}, \ldots, x_{n}\right)$ um polinômio central da álgebra $Q_{4}$. Pela Proposição 1.9 podemos assumir que $f$ é multilinear. Em particular, $f$ é homogêneo de grau $1 \mathrm{em}$ $x_{1}$. Assim, pelo Lema 2.4, $f$ pertence a $\left\langle\left[x_{1}, x_{2}\right]\left[x_{3}, x_{4}\right]\right\rangle^{T S}+T^{(3)}$. Consequentemente $f$ pertence ao $T$-subespaço gerado pelos polinômios $\left[x_{1}, x_{2}\right]\left[x_{3}, x_{4}\right]$ e $x_{1}\left[x_{2}, x_{3}, x_{4}\right]$.

A demonstração do lema seguinte é uma adaptação da demonstração do Lema 12 de [7].

Lema 2.7. Seja $F$ um corpo de característica $p \neq 0$ e $f=f\left(x_{1}, \ldots, x_{n}\right) \in F\langle X\rangle$ um polinômio homogêneo de grau $m_{1}$ em $x_{1}$, onde $m_{1}$ não é um múltiplo de $p$. Se $f+T^{(4)}$ for central em $Q_{4}$, então $f \in\left\langle\left[x_{1}, x_{2}\right]\left[x_{3}, x_{4}\right]\right\rangle^{T S}+T^{(3)}$.

Demonstração. Pela Proposição 1.16 não há perda de generalidade em supor que $f$ é multi-homogêneo de grau $m_{1}$ em $x_{1}$. Escreva $m_{1}=p q+r$ com $0<r<p$. 
Usando a base de $Q_{3}=F\langle X\rangle / T^{(3)}$ dada na Proposição 1.21, vemos que existe $g=g\left(x_{1}, \ldots, x_{n}\right) \in F\langle X\rangle$, multi-homogêneo de grau $r$ em $x_{1}$ tal que

$$
f+T^{(3)}=x_{1}^{p q} g+T^{(3)}
$$

Defina o endomorfismo $\varphi$ de $F\langle X\rangle$ por $\varphi\left(x_{1}\right)=1+x_{1}$ e $\varphi\left(x_{i}\right)=x_{i}$ se $i>1$. Por (2-8), temos

$$
\varphi(f)+T^{(3)}=\left(1+x_{1}^{p}\right)^{q} g\left(1+x_{1}, x_{2}, \ldots, x_{n}\right)+T^{(3)} .
$$

Observe que $C\left(Q_{4}\right)$ e $T^{(3)}$ são $T$-subespaços multi-homogêneos (Proposição 1.10 e Proposição 1.16, respectivamente), $\operatorname{logo} C\left(Q_{4}\right)+T^{(3)}$ é um $T$-subespaço multihomogêneo. Observe também que $g\left(x_{1}, \ldots, x_{n}\right)$ é a componente multi-homogênea de grau $r$ em $x_{1}$ do polinômio $\left(1+x_{1}^{p}\right)^{q} g\left(1+x_{1}, x_{2}, \ldots, x_{n}\right)$. Por $(2-9),\left(1+x_{1}^{p}\right)^{q} g(1+$ $\left.x_{1}, x_{2}, \ldots, x_{n}\right)$ pertence a $C\left(Q_{4}\right)+T^{(3)}$, logo $g$ pertence a $C\left(Q_{4}\right)+T^{(3)}$. Seja $h=$ $h\left(y_{1}, \ldots, y_{r}, x_{2}, \ldots, x_{n}\right)$ a linearização total de $g$ em $x_{1}$. Então $h \in C\left(Q_{4}\right)+T^{(3)}$ e segue do Lema 2.4 que $h \in\left\langle\left[x_{2}, x_{3}\right]\left[x_{4}, x_{5}\right]\right\rangle^{T S}+T^{(3)}$. Como

$$
h\left(x_{1}, \ldots, x_{1}, x_{2}, \ldots, x_{n}\right)=r ! g\left(x_{1}, \ldots, x_{n}\right)
$$

segue que $g \in\left\langle\left[x_{1}, x_{2}\right]\left[x_{3}, x_{4}\right]\right\rangle^{T S}+T^{(3)}$. Finalmente, como $x_{1}^{p}+T^{(3)}$ é central em $Q_{3}$ (Lema $1.22(\mathrm{i}))$ e $C\left(Q_{3}\right)$ é uma álgebra (Proposição 1.13), é claro que $x_{1}^{p q}+T^{(3)}$ é central em $Q_{3}$. Assim

$$
x_{1}^{p q}\left[x_{2}, x_{3}\right]\left[x_{4}, x_{5}\right]+T^{(3)}=\left[x_{1}^{p q} x_{2}, x_{3}\right]\left[x_{4}, x_{5}\right]+T^{(3)}
$$

Como $g \in\left\langle\left[x_{1}, x_{2}\right]\left[x_{3}, x_{4}\right]\right\rangle^{T S}+T^{(3)}$, segue de (2-8) e (2-10) que $f \in\left\langle\left[x_{1}, x_{2}\right]\left[x_{3}, x_{4}\right]\right\rangle^{T S}+$ $T^{(3)}$. O lema está demonstrado.

Lembrando que $q_{n}=q_{n}\left(x_{1}, \ldots, x_{2 n}\right)=q\left(x_{1}, x_{2}\right) \ldots q\left(x_{2 n-1}, x_{2 n}\right)$ onde $q\left(x_{1}, x_{2}\right)=x_{1}^{p-1}\left[x_{1}, x_{2}\right] x_{2}^{p-1}$, temos o seguinte:

Lema 2.8 (veja [22]). Seja $F$ um corpo de característica $p \neq 3$. Então $q_{n}+T^{(4)}$ é central na álgebra $Q_{4}=F\langle X\rangle / T^{(4)}$ se, e somente se $n \geq 2$.

Demonstração. Vamos mostrar que $q_{n}+T^{(4)}$ é central em $Q_{4}$ para cada $n \geq 2$. A prova será por indução sobre $n$. Primeiramente vamos mostrar que $q_{2}+T^{(4)}$ é central na álgebra $Q_{4}$, isto é, que $\left[q\left(x_{1}, x_{2}\right) q\left(x_{3}, x_{4}\right), x_{5}\right]=q\left(x_{1}, x_{2}\right)\left[q\left(x_{3}, x_{4}\right), x_{5}\right]+$ $\left[q\left(x_{1}, x_{2}\right), x_{5}\right] q\left(x_{3}, x_{4}\right)$ pertence a $T^{(4)}$; essa será a base da indução. Usando a igualdade 
$\left[a_{1} a_{2} \ldots a_{n}, a\right]=\sum_{i=1}^{n} a_{1} \ldots a_{i-1}\left[a_{i}, a\right] a_{i+1} \ldots a_{n}($ Lema $1.17(\mathrm{ii}))$, temos

$$
\begin{aligned}
{\left[q\left(x_{3}, x_{4}\right), x_{5}\right] } & =\left[x_{3}^{p-1}\left[x_{3}, x_{4}\right] x_{4}^{p-1}, x_{5}\right] \\
& =\left[x_{3}, x_{5}\right] x_{3}^{p-2}\left[x_{3}, x_{4}\right] x_{4}^{p-1}+\ldots+x_{3}^{p-1}\left[x_{3}, x_{4}, x_{5}\right] x_{4}^{p-1}+\ldots+ \\
& +x_{3}^{p-1}\left[x_{3}, x_{4}\right] x_{4}^{p-2}\left[x_{4}, x_{5}\right] \\
& =x_{3}^{p-2}\left[x_{3}, x_{5}\right]\left[x_{3}, x_{4}\right] x_{4}^{p-1}+\left[x_{3}, x_{5}, x_{3}^{p-2}\right]\left[x_{3}, x_{4}\right] x_{4}^{p-1}+\ldots+ \\
& +x_{3}^{p-1}\left[x_{3}, x_{4}, x_{5}\right] x_{4}^{p-1}+\ldots+x_{3}^{p-1}\left[x_{3}, x_{4}\right]\left[x_{4}, x_{5}\right] x_{4}^{p-2}+ \\
& +x_{3}^{p-1}\left[x_{3}, x_{4}\right]\left[x_{4}^{p-2},\left[x_{4}, x_{5}\right]\right] .
\end{aligned}
$$

Como $\left[x_{1}, x_{2}, x_{3}\right]\left[x_{4}, x_{5}\right] \in T^{(4)}$ (Corolário 1.33 (iii)) segue que todos os polinômios $\left[x_{3}, x_{5}, x_{3}^{p-2}\right]\left[x_{3}, x_{4}\right] x_{4}^{p-1}, \ldots, x_{3}^{p-1}\left[x_{3}, x_{4}\right]\left[x_{4}^{p-2},\left[x_{4}, x_{5}\right]\right]$ pertencem a $T^{(4)}$. Assim

$$
\begin{aligned}
{\left[q\left(x_{3}, x_{4}\right), x_{5}\right] } & \equiv x_{3}^{p-2}\left[x_{3}, x_{5}\right]\left[x_{3}, x_{4}\right] x_{4}^{p-1}+\ldots+x_{3}^{p-1}\left[x_{3}, x_{4}, x_{5}\right] x_{4}^{p-1}+\ldots+(2-1) \\
& +x_{3}^{p-1}\left[x_{3}, x_{4}\right]\left[x_{4}, x_{5}\right] x_{4}^{p-2}\left(\bmod T^{(4)}\right)
\end{aligned}
$$

Uma vez que $\left[x_{1}, x_{2}\right]\left[x_{3}, x_{4}\right]+T^{(4)}$ e $\left[x_{1}, x_{2}, x_{3}\right]+T^{(4)}$ são centrais em $Q_{4}$ (Lema 2.5), decorre de (2-11) que

$$
\begin{aligned}
q\left(x_{1}, x_{2}\right)\left[q\left(x_{3}, x_{4}\right), x_{5}\right] & \equiv x_{1}^{p-1}\left[x_{1}, x_{2}\right]\left[x_{3}, x_{5}\right]\left[x_{3}, x_{4}\right] x_{2}^{p-1} x_{3}^{p-2} x_{4}^{p-1}+\ldots+ \\
& +x_{1}^{p-1}\left[x_{1}, x_{2}\right]\left[x_{3}, x_{4}, x_{5}\right] x_{2}^{p-1} x_{3}^{p-1} x_{4}^{p-1}+ \\
& +x_{1}^{p-1}\left[x_{1}, x_{2}\right]\left[x_{3}, x_{4}\right]\left[x_{4}, x_{5}\right] x_{2}^{p-1} x_{3}^{p-1} x_{4}^{p-2} \quad\left(\bmod T^{(4)}\right) .
\end{aligned}
$$

O Corolário 1.33 (i) implica que todos os polinômios

$$
x_{1}^{p-1}\left[x_{1}, x_{2}\right]\left[x_{3}, x_{5}\right]\left[x_{3}, x_{4}\right] x_{2}^{p-1} x_{3}^{p-2} x_{4}^{p-1}, \ldots, x_{1}^{p-1}\left[x_{1}, x_{2}\right]\left[x_{3}, x_{4}\right]\left[x_{4}, x_{5}\right] x_{2}^{p-1} x_{3}^{p-1} x_{4}^{p-2}
$$

pertencem a $T^{(4)}$ e o Corolário 1.33 (iii) implica que o polinômio $x_{1}^{p-1}\left[x_{1}, x_{2}\right]\left[x_{3}, x_{4}, x_{5}\right] x_{2}^{p-1} x_{3}^{p-1} x_{4}^{p-1}$ também pertence a $T^{(4)}$. Assim, por (2-12) temos que $q\left(x_{1}, x_{2}\right)\left[q\left(x_{3}, x_{4}\right), x_{5}\right]$ pertence a $T^{(4)}$. É fácil ver que $\left[q\left(x_{1}, x_{2}\right), x_{5}\right] q\left(x_{3}, x_{4}\right)$ também pertence a $T^{(4)}$. Portanto

$$
\left[q_{2}, x_{5}\right]=\left[q\left(x_{1}, x_{2}\right) q\left(x_{3}, x_{4}\right), x_{5}\right]=q\left(x_{1}, x_{2}\right)\left[q\left(x_{3}, x_{4}\right), x_{5}\right]+\left[q\left(x_{1}, x_{2}\right), x_{5}\right] q\left(x_{3}, x_{4}\right)
$$

pertence a $T^{(4)}$, isto é, $q_{2}+T^{(4)}$ é central em $Q_{4}$.

Agora, suponha por hipótese de indução que $q_{m}+T^{(4)}$ é central de $Q_{4}$ para cada $m$ satisfazendo $2 \leq m<n$. Como $C\left(Q_{4}\right)$ é uma álgebra (Proposição 1.13) e ambos $q_{n-2}$ e $q_{2}$ pertencem a $C\left(Q_{4}\right)$, segue que $q_{n}=q_{n-2} q_{2}$ também pertence a $C\left(Q_{4}\right)$. Isso completa a indução. 
Para finalizar a demonstração, resta mostrar apenas que $q_{1}+T^{(4)}=$ $x_{1}^{p-1}\left[x_{1}, x_{2}\right] x_{2}^{p-1}+T^{(4)}$ não é central em $Q_{4}$. De fato, como $\left[x_{1}, x_{2}\right]$ é uma componente multi-homogênea do polinômio $\left(x_{1}+1\right)^{p-1}\left[x_{1}+1, x_{2}+1\right]\left(x_{2}+1\right)^{p-1}$, se $x_{1}\left[x_{1}, x_{2}\right] x_{2}+T^{(4)}$ fosse central em $Q_{4}$, então $\left[x_{1}, x_{2}\right]+T^{(4)}$ seria central em $Q_{4}$, uma contradição. Logo $q_{1}+T^{(4)}$ não é central em $Q_{4}$. O lema está demonstrado.

Lema 2.9. Seja $F$ um corpo de característica 2. Então $x_{0}^{n}+T^{(4)}$ é central na álgebra $Q_{4}=F\langle X\rangle / T^{(4)}$ se, e somente se $n$ é um múltiplo de 4 .

Demonstração. Usando o fato de que $\operatorname{char}(F)=2$, um cálculo direto mostra que

$$
\left[x_{0}^{4}, x_{1}\right]=\left[x_{0}, x_{1}, x_{0}, x_{0}^{2}\right]
$$

Assim $\left[x_{0}^{4}, x_{1}\right]$ pertence a $T^{(4)}$, isto é $x_{0}^{4}+T^{(4)}$ é central em $Q_{4}$. Sendo $C\left(Q_{4}\right)$ uma álgebra (Proposição 1.13) segue que $x_{0}^{4 t}+T^{(4)}$ é central em $Q_{4}$ para cada $t \in \mathbb{N}$. Agora suponha que $x_{0}^{n}+T^{(4)}$ seja central em $Q_{4}$. Vamos mostrar que $n=4 t$ para algum $t \in \mathbb{N}$. É suficiente mostrar que os elementos $x_{0}^{2}+T^{(4)}, x_{0}^{3}+T^{(4)}, x_{0}^{4 t+1}+T^{(4)}, x_{0}^{4 t+2}+T^{(4)} \mathrm{e}$ $x_{0}^{4 t+3}+T^{(4)}$ não são centrais em $Q_{4}$ para qualquer $t \in \mathbb{N}$.

Como $\left[x_{0}^{2}, x_{1}\right]=\left[x_{0}, x_{1}, x_{0}\right]$ possui grau 3 , segue que $\left[x_{0}^{2}, x_{1}\right]$ não pertence a $T^{(4)}$, isto é $x_{0}^{2}+T^{(4)}$ não é central em $Q_{4}$. Observe que

$$
\left[x_{0}^{3}, x_{1}\right]=x_{0}^{2}\left[x_{0}, x_{1}\right]+\left[x_{0}, x_{1}, x_{0}\right] x_{0}
$$

Suponha que $\left[x_{0}^{3}, x_{1}\right] \in T^{(4)}$. Como $\left[x_{0}, x_{1}\right]$ é uma componente multi-homogênea do polinômio $\left(x_{0}+1\right)^{2}\left[x_{0}, x_{1}\right]+\left[x_{0}, x_{1}, x_{0}\right]\left(x_{0}+1\right)$, segue que $\left[x_{0}, x_{1}\right] \in T^{(4)}$. Mas isso não é possível porque o polinômio $\left[x_{0}, x_{1}\right]$ possui grau 2. Logo $x_{0}^{3}+T^{(4)}$ não é central em $Q_{4}$. Como $x_{0}^{4 t}+T^{(4)}$ é central em $Q_{4}$, temos

$$
\left[x_{0}^{4 t+1}, x_{1}\right]=x_{0}^{4 t}\left[x_{0}, x_{1}\right]+\left[x_{0}^{4 t}, x_{1}\right] x_{0} \equiv x_{0}^{4 t}\left[x_{0}, x_{1}\right] \quad\left(\bmod T^{(4)}\right) .
$$

Como $\left[x_{0}, x_{1}\right]$ é uma componente multi-homogênea do polinômio $\left(x_{0}+1\right)^{4 t}\left[x_{0}, x_{1}\right]$, se $\left[x_{0}^{4 t+1}, x_{1}\right] \in T^{(4)}$, teríamos $\left[x_{0}, x_{1}\right] \in T^{(4)}$, o que é uma contradição. Portanto $x_{0}^{4 t+1}+T^{(4)}$ não é central em $Q_{4}$. Agora

$$
\begin{aligned}
{\left[x_{0}^{4 t+2}, x_{1}\right] } & =x_{0}^{4 t}\left[x_{0}^{2}, x_{1}\right]+\left[x_{0}^{4 t}, x_{1}\right] x_{0}^{2} \\
& \equiv x_{0}^{4 t}\left[x_{0}^{2}, x_{1}\right] \quad\left(\bmod T^{(4)}\right) \\
& =x_{0}^{4 t}\left[x_{0}, x_{1}, x_{0}\right] .
\end{aligned}
$$

Como $\left[x_{0}, x_{1}, x_{0}\right]$ é uma componente multi-homogênea do polinômio $\left(x_{0}+\right.$ $1)^{4 t}\left[x_{0}, x_{1}, x_{0}\right]$, se $\left[x_{0}^{4 t+2}, x_{1}\right] \in T^{(4)}$, teríamos $\left[x_{0}, x_{1}\right] \in T^{(4)}$, o que é uma contra- 
dição. Portanto $x_{0}^{4 t+3}+T^{(4)}$ não é central em $Q_{4}$. Por último, usando (2-13) temos

$$
\begin{aligned}
{\left[x_{0}^{4 t+3}, x_{1}\right] } & =x_{0}^{4 t}\left[x_{0}^{3}, x_{1}\right]+\left[x_{0}^{4 t}, x_{1}\right] x_{0}^{3} \\
& \equiv x_{0}^{4 t}\left[x_{0}^{3}, x_{1}\right] \quad\left(\bmod T^{(4)}\right) \\
& =x_{0}^{4 t}\left(x_{0}^{2}\left[x_{0}, x_{1}\right]+\left[x_{0}, x_{1}, x_{0}\right] x_{0}\right) \\
& =x_{0}^{4 t+2}\left[x_{0}, x_{1}\right]+x_{0}^{4 t}\left[x_{0}, x_{1}, x_{0}\right] x_{0}
\end{aligned}
$$

Como $\left[x_{0}, x_{1}\right]$ é uma componente multi-homogênea do polinômio $\left(x_{0}+1\right)^{4 t+2}\left[x_{0}, x_{1}\right]+$ $\left(x_{0}+1\right)^{4 t}\left[x_{0}, x_{1}, x_{0}\right]\left(x_{0}+1\right)$, se $\left[x_{0}^{4 t+3}, x_{1}\right] \in T^{(4)}$, teríamos $\left[x_{0}, x_{1}\right]+\left[x_{0}, x_{1}, x_{0}\right] \in T^{(4)}, \mathrm{o}$ que é uma contradição. Portanto $x_{0}^{4 t+3}+T^{(4)}$ não é central em $Q_{4}$. Isso conclui a demonstração.

\section{Demonstração do Teorema 2.1.}

Demonstração. Vamos verificar primeiramente que

$$
x_{1}\left[x_{2}, x_{3}, x_{4}\right], x_{0}^{4}, x_{0}^{2} q_{2}, x_{0}^{2} q_{3}, x_{0}^{2} q_{4}, \ldots, x_{0}^{2} q_{n}, \ldots
$$

são polinômios centrais de $Q_{4}$. Segue do Lema 2.5 e do Lema 2.9 que os elementos $x_{1}\left[x_{2}, x_{3}, x_{4}\right]+T^{(4)}$ e $x_{0}+T^{(4)}$ são centrais em $Q_{4}$, respectivamente. Resta mostrar que $x_{0}^{2} q_{n}+T^{(4)}$ é central em $Q_{4}$ para cada $n \geq 2$. De fato, usando a igualdade $[a b, c]=a[b, c]+[a, c] b$ temos

$$
\begin{aligned}
{\left[x_{0}^{2} q_{n}, x_{2 n+1}\right] } & =x_{0}^{2}\left[q_{n}, x_{2 n+1}\right]+\left[x_{0}^{2}, x_{2 n+1}\right] q_{n} \\
& =x_{0}^{2}\left[q_{n}, x_{2 n+1}\right]+2 x_{0}\left[x_{0}, x_{2 n+1}\right] q_{n}+\left[x_{0}, x_{2 n+1}, x_{0}\right] q_{n}
\end{aligned}
$$

Como $\left[x_{0}, x_{2 n+1}, x_{0}\right]+T^{(4)}$ é central em $Q_{4}$, segue do Corolário 1.33 (ii) (ou (iii)) que $\left[x_{0}, x_{2 n+1}, x_{0}\right] q_{n} \in T^{(4)}$. Pelo Lema 2.8, temos $x_{0}^{2}\left[q_{n}, x_{2 n+1}\right] \in T^{(4)}$. Sendo $\operatorname{char}(F)=2$, segue de $(2-15)$ que $\left[x_{0}^{2} q_{n}, x_{2 n+1}\right] \in T^{(4)}$. Portanto $x_{0}^{2} q_{n}+T^{(4)}$ é central em $Q_{4}$ para cada $n \geq 2$.

Vamos mostrar que os polinômios da forma (2-14) geram $C\left(Q_{4}\right)$ como $T$ subespaço. Seja $f=f\left(x_{1}, \ldots, x_{n}\right)$ um polinômio central de $Q_{4}$. Podemos assumir que $f \notin T^{(3)}$ pois $T^{(3)}$ é gerado como $T$-subespaço pelo polinômio $x_{1}\left[x_{2}, x_{3}, x_{4}\right]$, que está entre aqueles de (2-14). Pela Proposição 1.16, podemos assumir também que $f$ é multi-homogêneo de grau $m_{i}$ em cada variável $x_{i}(i=1, \ldots, n)$.

Suponha que o grau de alguma variável $x_{i}$ não seja divisível por 2 . Reenumerando as variáveis $x_{i}$, podemos assumir, sem perda de generalidade que $i=1$. Assim, pelo Lema 2.7, $f$ pertence ao $T$-subespaço gerado pelos polinômios $\left[x_{1}, x_{2}\right]\left[x_{3}, x_{4}\right]$ e $x_{1}\left[x_{2}, x_{3}, x_{4}\right]$. Segue do Lema 1.31 que o polinômio $\left[x_{1}, x_{2}\right]\left[x_{3}, x_{4}\right]$ pertence ao $T$ - 
subespaço gerado pelo polinômio $x_{0}^{2} q_{2}$. Logo $f$ pertence ao $T$-subespaço gerado pelos polinômios da forma (2-14).

Agora suponha que todos os graus $m_{i}$ das variáveis $x_{i}$ sejam divisíveis por 2. Como $x_{0}^{2}+T^{(3)}$ e $\left[x_{1}, x_{2}\right]+T^{(3)}$ são centrais em $Q_{3}$, segue da Proposição 1.21 que $f+T^{(3)}$ é uma combinação linear de elementos da forma

$$
x_{i_{1}}^{2 r_{1}} \ldots x_{i_{k}}^{2 r_{k}} x_{j_{1}}\left[x_{j_{1}}, x_{j_{2}}\right] x_{j_{2}} \ldots x_{j_{2 l-1}}\left[x_{j_{2 l-1}}, x_{j_{2 l}}\right] x_{j_{2 l}}+T^{(3)}
$$

onde $k \geq 0, l \geq 0,1 \leq i_{1}<\ldots<i_{k} \leq n$ e $1 \leq j_{1}<\ldots<j_{2 l} \leq n$.

Afirmação 1: $l \neq 1$ para cada elemento de (2-16). Suponha o contrário, que algum elemento de (2-16) ocorra com $l=1$. Escolha dentre os elementos $\operatorname{com} l=1$ aquele com $j_{1}$ minimal e coeficiente $\alpha \in F$ (não nulo). Denote tal elemento por

$$
\alpha x_{i_{1}}^{2 r_{1}} \ldots x_{i_{k}}^{2 r_{k}} x_{j_{1}}\left[x_{j_{1}}, x_{j_{2}}\right] x_{j_{2}}+T^{(3)}
$$

Defina o endomorfismo $\varphi$ de $F\langle X\rangle$ por $\varphi\left(x_{j_{s}}\right)=x_{j_{s}}$ se $s \in\{1,2\}$ e $\varphi\left(x_{j_{s}}\right)=1$ se $s \notin\{1,2\}$. Então segue de (2-16) e (2-17) que

$$
\begin{aligned}
\varphi(f)+T^{(3)} & =\alpha x_{j_{1}}^{2 r_{1}} x_{j_{2}}^{2 r_{2}} x_{j_{1}}\left[x_{j_{1}}, x_{j_{2}}\right] x_{j_{2}}+T^{(3)}+\beta x_{j_{1}}^{2\left(r_{1}+1\right)} x_{j_{2}}^{2\left(r_{2}+1\right)}+T^{(3)} \\
& =\alpha x_{j_{1}}^{2 r_{1}+1} x_{j_{2}}^{2 r_{2}+1}\left[x_{j_{1}}, x_{j_{2}}\right]+\beta x_{j_{1}}^{2\left(r_{1}+1\right)} x_{j_{2}}^{2\left(r_{2}+1\right)}+T^{(3)}
\end{aligned}
$$

onde $\beta \in F$ com $\beta \geq 0$. Como $T^{(3)} \subseteq C\left(Q_{4}\right)$, segue de (2-18) que $g\left(x_{j_{1}}, x_{j_{2}}\right)=$ $\alpha x_{j_{1}}^{2 r_{1}+1} x_{j_{2}}^{2 r_{2}+1}\left[x_{j_{1}}, x_{j_{2}}\right]+\beta x_{j_{1}}^{2\left(r_{1}+1\right)} x_{j_{2}}^{2\left(r_{2}+1\right)}$ é um polinômio central de $Q_{4}$. Uma vez que $C\left(Q_{4}\right)$ é multi-homogêneo e $\left[x_{j_{1}}, x_{j_{2}}\right]+x_{j_{1}} x_{j_{2}}=x_{j_{2}} x_{j_{1}}$ é uma componente multihomogênea do polinômio $g\left(x_{j_{1}}+1, x_{j_{2}}+1\right)$, segue que $x_{j_{2}} x_{j_{1}}$ é um polinômio central de $Q_{4}$. Mas isso não é possível porque todo polinômio central de $Q_{4}$ possui grau no mínimo 3. Portanto todos elementos de $(2-16)$ são tais que $l \neq 1$.

Afirmação 2. Cada elemento de (2-16) com $l \geq 2$ é uma combinação linear de elementos da forma

$$
b^{2} q_{s}\left(x_{j_{1}}, \ldots, x_{j_{2 s}}\right)+T^{(3)}, s \geq 2, b \in F\langle X\rangle .
$$

De fato, usando a igualdade $x_{1}^{2} x_{2}^{2}=\left(x_{1} x_{2}\right)^{2}+x_{1}\left[x_{1}, x_{2}\right] x_{2}$ em (2-16), não é difícil verificar a afirmação.

Afirmação 3: O elemento de (2-16) com $l=0$ é um múltiplo escalar do elemento $a^{4}+T^{(3)}$ para algum $a \in F\langle X\rangle$. De fato, segue da Afirmação 1 que os elementos (2-16) são tais que $l=0$ ou $l \geq 2$. Assim, pela Afirmação $2, f+T^{(3)}$ é a soma do elemento

$$
\gamma x_{1}^{2 r_{1}} \ldots x_{n}^{2 r_{n}}+T^{(3)}, \gamma \in F
$$


com uma combinação linear de elementos da forma (2-19). Se $\gamma=0$, então vemos que $f$ pertence ao $T$-subespaço gerado pelos polinômios $x_{1}\left[x_{2}, x_{3}, x_{4}\right]$ e $x_{0}^{2} q_{n}, n \geq 2$, que estão entre aqueles de (2-14). Assim podemos supor $\gamma \neq 0$. Como $f+T^{(4)}$ e $b^{2} q_{s}\left(x_{j_{1}}, \ldots, x_{j_{2 s}}\right)+T^{(4)}$ são centrais em $Q_{4}$, segue que $\gamma x_{1}^{2 r_{1}} \ldots x_{n}^{2 r_{n}}+T^{(4)}$ é central em $Q_{4}$. Agora para cada $i=1, \ldots, n$, defina os endomorfismos $\phi_{i}$ de $F\langle X\rangle$ por $\phi_{i}\left(x_{i}\right)=x_{i}$ e $\phi_{i}\left(x_{s}\right)=1$ se $s \neq i$. Segue de $(2-20)$ que $\phi_{i}(f)+T^{(3)}=\gamma x_{i}^{2 r_{i}}+T^{(3)}$. Como $T^{(3)} \subseteq C\left(Q_{4}\right)$ e $\gamma \neq 0$, temos que $x_{i}^{2 r_{i}}+T^{(4)}$ é central em $Q_{4}$. Pelo Lema 2.9 temos $2 r_{i}=4 t_{i}$ para algum $t_{i} \in \mathbb{N}$. Logo (2-20) pode ser escrito como

$$
\gamma x_{1}^{4 t_{1}} \ldots x_{n}^{4 t_{n}}+T^{(3)}
$$

Como $\left(x_{1} x_{2}\right)^{4} \equiv x_{1}^{4} x_{2}^{4}\left(\bmod T^{(3)}\right)($ Lema $1.22($ iii $))$, segue que $\gamma x_{1}^{4 t_{1}} \ldots x_{n}^{4 t_{n}}+T^{(3)}=$ $\gamma\left(x_{1}^{t_{1}} \ldots x_{n}^{t_{n}}\right)^{4}+T^{(3)}$. Pondo $b=\left(x_{1}^{t_{1}} \ldots x_{n}^{t_{n}}\right)$ a Afirmação 3 segue. Agora, segue de (216) e das Afirmações 1 à 3 que $f$ pertence ao $T$-subespaço gerado pelos polinômios (2-14). A demonstração está completa.

\section{Demonstração do Teorema 2.2.}

Demonstração. Primeiramente vamos mostrar que (2-1)-(2-2) são polinômios centrais de $Q_{4}$. Como $C\left(Q_{4}\right)$ é uma $T$-subálgebra de $F\langle X\rangle$ (Proposição 1.13), é suficiente mostrar que

$$
\left[x_{1}, x_{2}\right]\left[x_{3}, x_{4}\right], x_{1}\left[x_{2}, x_{3}, x_{4}\right], x_{0}^{p} \text { e } q_{n}, n \geq 2
$$

são polinômios centrais de $Q_{4}$; mas isso segue diretamente do Lema 2.5, do Lema 1.22 (i) e do Lema 2.8.

Vamos mostrar agora que os polinômios (2-1)-(2-2) geram $C\left(Q_{4}\right)$ como espaço vetorial. Seja $f=f\left(x_{1}, \ldots, x_{n}\right)$ um polinômio central de $Q_{4}$. Pela Proposição 1.16 podemos assumir que $f$ é um polinômio multi-homogêneo de grau $m_{i}$ em cada variável $x_{i}$. Se $f \in T^{(3)}$ não é difícil ver que $f$ é uma combinação linear de polinômios da forma $a_{1}\left[a_{2}, a_{3}, a_{4}\right]$ com $a_{i} \in F\langle X\rangle$ (que estão entre aqueles de (i)). Assim podemos assumir que $f \notin T^{(3)}$.

Considere o caso em que o grau $m_{i}$ de alguma variável $x_{i}$ não seja múltiplo de $p$. Reenumerando as variáveis $x_{i}$, podemos assumir, sem perda de generalidade, que $i=1$. Pelo Lema 2.7, temos que $f$ pertence ao $T$-subespaço gerado pelos polinômios $\left[x_{1}, x_{2}\right]\left[x_{3}, x_{4}\right]$ e $x_{1}\left[x_{2}, x_{3}, x_{4}\right]$ e disso decorre que $f$ é uma combinação linear de polinômios de (2-1).

Agora, considere o caso em que os graus $m_{i}$ de todas as variáveis do polinômio $f$ sejam divisíveis por $p$. Como $x_{0}^{p}+T^{(3)}$ e $\left[x_{1}, x_{2}\right]+T^{(3)}$ são centrais em $Q_{3}$, segue da Proposição 1.21 que $f+T^{(3)}$ pode ser escrito como uma combinação 
linear de elementos da forma

$$
x_{i_{1}}^{p r_{1}} \ldots x_{i_{k}}^{p r_{k}} x_{j_{1}}^{p-1}\left[x_{j_{1}}, x_{j_{2}}\right] x_{j_{2}}^{p-1} \ldots x_{j_{2 l-1}}^{p-1}\left[x_{j_{2 l-1}}, x_{j_{2 l}}\right] x_{j_{2 l}}^{p-1}+T^{(3)}
$$

onde $k, l \geq 0, r_{s}>0,1 \leq i_{1}<\ldots<i_{k} \leq n, 1 \leq j_{1}<\ldots<j_{2 l} \leq n$. Afirmamos que $l \neq 1$ para cada elemento em (2-22). Suponha o contrário, que algum elemento de (2-22) possua $l=1$; e dentre todos esses, escolha aquele com $j_{1}$ minimal. Tal elemento se escreve como

$$
x_{i_{1}}^{p r_{1}} \ldots x_{i_{k}}^{p r_{k}} x_{j_{1}}^{p-1}\left[x_{j_{1}}, x_{j_{2}}\right] x_{j_{2}}^{p-1}+T^{(3)} .
$$

Defina o endomorfismo $\varphi$ de $F\langle X\rangle$ por $\varphi\left(x_{j_{s}}\right)=x_{j_{s}}$ se $s \in\{1,2\}$ e $\varphi\left(x_{j_{s}}\right)=1$ se $s \notin\{1,2\}$. Então por $(2-22)$ e (2-23) temos

$$
\varphi(f)+T^{(3)}=\alpha x_{j_{1}}^{p r_{j_{1}}} x_{j_{2}}^{p r_{j_{2}}} x_{j_{1}}^{p-1}\left[x_{j_{1}}, x_{j_{2}}\right] x_{j_{2}}^{p-1}+T^{(3)},
$$

onde $\alpha \in F$ é não nulo. Segue do Lema 1.33 (iii) que $x_{1}\left[x_{2}, x_{3}, x_{4}\right]+T^{(4)}$ é central em $Q_{4}$. Consequentemente $T^{(3)} \subseteq C\left(Q_{4}\right)$. Assim, por (2-24) segue que $g\left(x_{j_{1}}, x_{j_{2}}\right)=$ $x_{j_{1}}^{p r_{j_{1}}} x_{j_{2}}^{p_{j_{2}}} x_{j_{1}}^{p-1}\left[x_{j_{1}}, x_{j_{2}}\right] x_{j_{2}}^{p-1}$ é um polinômio central de $Q_{4}$. Como $\left[x_{j_{1}}, x_{j_{2}}\right]$ é uma componente multi-homogênea do polinômio $g\left(x_{j_{1}}+1, x_{j_{2}}+1\right)=\left(x_{j_{1}}+1\right)^{\operatorname{pr}_{j_{1}}}\left(x_{j_{2}}+\right.$

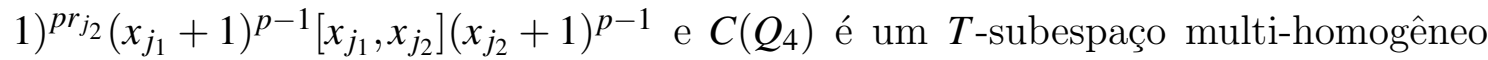
(Proposição 1.16) segue que $\left[x_{j_{1}}, x_{j_{2}}\right]+T^{(4)}$ é central em $Q_{4}$, o que é uma contradição porque $\left[x_{j_{1}}, x_{j_{2}}, x_{j_{3}}\right] \notin T^{(4)}$.

Desse modo, $f+T^{(3)}$ pode ser escrito como uma combinação linear de elementos de (2-22) com $l=0$ ou $l \geq 2$. Como $T^{(3)}$ é gerado como $T$-subespaço por $x_{1}\left[x_{2}, x_{3}, x_{4}\right]$, segue que $f$ pode ser escrito como uma combinação linear de polinômios de (2-1)-(2-2). Isso conclui a demonstração.

Conforme mostrado por Grishin [[22], Teorema 2] (veja o Teorema 0.2), quando $\operatorname{char}(F)=p>3, C\left(Q_{4}\right)$ é gerado como $T$-subespaço pelos polinômios

$$
x_{1}\left[x_{2}, x_{3}, x_{4}\right], x_{0}^{p}, x_{0}^{p} q_{2}, \ldots, x_{0}^{p} q_{n}, \ldots
$$

Esse resultado é uma consequência do Teorema 2.2. De fato, seja $Q$ o $T$ subespaço gerado pelos polinômios (2-25). Como os polinômios (2-25) estão entre aqueles de (2-1)-(2-2), segue que $Q \subseteq C\left(Q_{4}\right)$.

Para obter a inclusão contrária, é suficiente mostrar que os polinômios (21)-(2-2) pertencem a $Q$. É claro $a_{1}\left[a_{2}, a_{3}, a_{4}\right]$ pertence a $Q$. Segue do (Lema 1.31) que $\left[x_{1}, x_{2}\right]\left[x_{3}, x_{4}\right]$ pertence ao $T$-subespaço gerado pelo polinômio $x_{0}^{p} q_{2}$. Assim os polinômios de (2-1) pertencem a $Q$. 
Como $\left(x_{1} x_{2}\right)^{p} \equiv x_{1}^{p} x_{2}^{p}\left(\bmod T^{(3)}\right)$ (Lema $1.22($ ii) $)$, não é difícil ver que os polinômios da forma (2-25) pertencem ao $T$-subespaço gerado pelos polinômios $x_{1}\left[x_{2}, x_{3}, x_{4}\right], x_{0}^{p}$ e $x_{0}^{p} q_{n}, n \geq 2$. Logo os polinômios da forma (2-25) pertencem $Q$, consequentemente $C\left(Q_{4}\right) \subseteq Q$.

\subsection{O caso de característica 3}

O objetivo principal desta seção é dar uma descrição dos polinômios centrais da álgebra $Q_{4}$ quando $F$ é um corpo de característica 3. Mais precisamente, provaremos o nosso primeiro resultado principal:

Teorema 2.10. Seja $F$ um corpo de característica 3. Então $C\left(Q_{4}\right)$, o espaço vetorial dos polinômios centrais da álgebra $Q_{4}=F\langle X\rangle / T^{(4)}$ é gerado, como $T$-subespaço de $F\langle X\rangle$, pelos polinômios

(i) $x_{1} \omega\left(x_{2}, x_{3}, x_{4}, x_{5}\right),\left[x_{1}, x_{2}\right]\left[x_{3}, x_{4}\right]-x_{1}\left[x_{2}, x_{3}, x_{4}\right], x_{1}\left[x_{2}, x_{3}, x_{4}, x_{5}\right]$,

(ii) $x_{0}^{3}, x_{0}^{3} q_{3}, x_{0}^{3} q_{6}, \ldots, x_{0}^{3} q_{3 n}, \ldots$,

(iii) $x_{0}^{3} u_{0}, x_{0}^{3} u_{1}, x_{0}^{3} u_{2}, \ldots, x_{0}^{3} u_{n}, \ldots$

\subsubsection{Uma base para o espaço vetorial $Q_{4}$}

Seja $T^{(3,2)}$ o $T$-ideal de $F\langle X\rangle$ gerado pelos polinômios $\left[x_{1}, x_{2}, x_{3}, x_{4}\right]$ e $\left[x_{1}, x_{2}, x_{3}\right]\left[x_{4}, x_{5}\right]$. Claramente $T^{(4)} \subseteq T^{(3,2)}$. Se $F$ possui característica $\neq 3$, então $T^{(4)}=T^{(3,2)}$ pois $\left[x_{1}, x_{2}, x_{3}\right]\left[x_{4}, x_{5}\right]$ pertence a $T^{(4)}$ (Corolário 1.33). Quando $F$ possui característica 3 , foi mostrado por Krasilnikov [33] que $\left[x_{1}, x_{2}, x_{3}\right]\left[x_{4}, x_{5}\right]$ não pertence a $T^{(4)}$. Assim $T^{(4)} \subsetneq T^{(3,2)}$.

Uma base para $T^{(3,2)} / T^{(4)}$ foi encontrada por Deryabina e Krasilnikov [11].

Teorema 2.11 (veja [11],Teorema 1.2). Seja F um corpo de característica 3. Então o espaço vetorial $T^{(3,2)} / T^{(4)}$ possui uma base

$$
\begin{gathered}
x_{i_{1}} x_{i_{2}} \ldots x_{i_{k}}\left[x_{j_{1}}, x_{j_{2}}\right] \ldots\left[x_{j_{2 l-1}}, x_{j_{2 l}}\right]\left[x_{j_{2 l+1}}, x_{j_{2 l+2}}, x_{j_{2 l+3}}\right]+T^{(4)} \\
k \geq 0, l \geq 1, i_{1} \leq i_{2} \leq \ldots \leq i_{k}, j_{1}<j_{2}<\ldots<j_{2 l+3} .
\end{gathered}
$$

Lembremos que $E_{n}$ é a álgebra de Grassmann (unitária) gerada por $e_{1}, \ldots, e_{n}$. O lema seguinte é bem conhecido, veja [12, Lema 2], [14, página 16],[21, Lema 3], [43, Corolário 4] e [44].

Lema 2.12 (veja $[21,44]$ ). Seja $F$ um corpo de característica $\neq 2$. Então $T^{(4)} \subseteq$ $I d\left(E_{2} \otimes E_{2}\right)$. 
Demonstração. Devemos mostrar que a álgebra $E_{2} \otimes E_{2}$ satisfaz a identidade polinomial $\left[x_{1}, x_{2}, x_{3}, x_{4}\right]=0$. Considere as seguintes bases da álgebra $E_{2}$ :

$$
\begin{aligned}
& 1, e_{1}, e_{2}, e_{1} e_{2}, \\
& 1, h_{1}, h_{2}, h_{1} h_{2} .
\end{aligned}
$$

Como o polinômio $\left[x_{1}, x_{2}, x_{3}, x_{4}\right]$ é multilinear, é suficiente avaliá-lo na base de $E_{2} \otimes E_{2}$, isto é, nos elementos $a \otimes b$, onde $a$ é um elemento de (2-26) e $b$ é um elemento de (2-27). Sejam $a_{1}, \ldots, a_{4}, b_{1}, \ldots, b_{4}$ elementos quaisquer de $E_{2}$. Observe que

$$
\begin{aligned}
{\left[a_{1} \otimes b_{1}, a_{2} \otimes b_{2}\right] } & =a_{1} a_{2} \otimes b_{1} b_{2}-a_{2} a_{1} \otimes b_{2} b_{1} \\
& =a_{1} a_{2} \otimes b_{1} b_{2}-a_{2} a_{1} \otimes b_{1} b_{2}+a_{2} a_{1} \otimes b_{1} b_{2}-a_{2} a_{1} \otimes b_{2} b_{1} \\
& =\left[a_{1}, a_{2}\right] \otimes b_{1} b_{2}+a_{2} a_{1} \otimes\left[b_{1}, b_{2}\right] .
\end{aligned}
$$

Usando (2-28) duas vezes e observando que $\left[x_{1}, x_{2}\right]$ é um polinômio central de $E_{2}$, podemos escrever

$$
\begin{aligned}
{\left[a_{1} \otimes b_{1}, a_{2} \otimes b_{2}, a_{3} \otimes b_{3}, a_{4} \otimes b_{4}\right] } & =\left[a_{1}, a_{2}\right]\left[a_{3}, a_{4}\right] \otimes\left[b_{1} b_{2}, b_{3}\right] b_{4}+ \\
& +\left[a_{1} a_{2}, a_{3}\right] a_{4} \otimes\left[b_{1}, b_{2}\right]\left[b_{3}, b_{4}\right] .
\end{aligned}
$$

Como $\left[x_{1}, x_{2}\right]\left[x_{3}, x_{4}\right]$ é um polinômio multilinear, usando a base (2-26) não é difícil ver que $E_{2}$ satisfaz a identidade polinomial $\left[x_{1}, x_{2}\right]\left[x_{3}, x_{4}\right]=0$. Assim, voltando a (2$29)$ vemos que $\left[a_{1}, a_{2}\right]\left[a_{3}, a_{4}\right]=0$ e $\left[b_{1}, b_{2}\right]\left[b_{3}, b_{4}\right]=0$. Portanto $\left[a_{1} \otimes b_{1}, a_{2} \otimes b_{2}, a_{3} \otimes\right.$ $\left.b_{3}, a_{4} \otimes b_{4}\right]=0$ e $\left[x_{1}, x_{2}, x_{3}, x_{4}\right]=0$ é uma identidade polinomial para $E_{2} \otimes E_{2}$.

Lema 2.13. Seja $F$ um corpo de característica $\neq 2$. Então o polinômio $\left[x_{1}, x_{2}\right]\left[x_{2}, x_{3}\right]$ não pertence a $T^{(4)}$.

Demonstração. Suponha que $\left[x_{1}, x_{2}\right]\left[x_{2}, x_{3}\right]$ pertença a $T^{(4)}$. Como

$$
\left[x_{1}, x_{2}+x_{3}\right]\left[x_{2}+x_{3}, x_{4}\right]=\omega\left(x_{1}, x_{2}, x_{3}, x_{4}\right)+\left[x_{1}, x_{2}\right]\left[x_{2}, x_{4}\right]+\left[x_{1}, x_{3}\right]\left[x_{3}, x_{4}\right]
$$

segue que $\omega\left(x_{1}, x_{2}, x_{3}, x_{4}\right)$ também pertence a $T^{(4)}$. Mas

$$
\omega\left(e_{1} \otimes 1, e_{2} \otimes 1,1 \otimes h_{1}, 1 \otimes h_{2}\right)=4 e_{1} e_{2} \otimes h_{1} h_{2} \neq 0
$$

em $E_{2} \otimes E_{2}$, o que contraria o Lema 2.12. Portanto $\left[x_{1}, x_{2}\right]\left[x_{2}, x_{3}\right]$ não pertence a $T^{(4)}$.

Quando $F$ possui característica 0 , uma base para o espaço vetorial $Q_{4}$ foi encontrada independentemente por Volichenko [44] e Gordienko [21]. À seguir 
exibimos uma base para o espaço vetorial $Q_{4}$ quando $F$ possui característica 3.

Proposição 2.14. Seja F um corpo de característica 3. Então a imagem dos polinômios

$$
\begin{gathered}
x_{i_{1}} x_{i_{2}} \ldots x_{i_{k}}, i_{1} \leq i_{2} \leq \ldots \leq i_{k} \\
x_{i_{1}} x_{i_{2}} \ldots x_{i_{k}}\left[x_{j_{1}}, x_{j_{2}}\right], i_{1} \leq i_{2} \leq \ldots \leq i_{k}, j_{1}<j_{2}, \\
x_{i_{1}} x_{i_{2}} \ldots x_{i_{k}}\left[x_{j_{1}}, x_{j_{2}}, x_{j_{2}}\right], i_{1} \leq i_{2} \leq \ldots \leq i_{k}, j_{1} \neq j_{2}, \\
x_{i_{1}} x_{i_{2}} \ldots x_{i_{k}}\left[x_{j_{1}}, x_{j_{2}}, x_{j_{3}}\right], i_{1} \leq i_{2} \leq \ldots \leq i_{k}, j_{1}>j_{2}<j_{3}, \\
x_{i_{1}} x_{i_{2}} \ldots x_{i_{k}}\left[x_{j_{1}}, x_{j_{2}}\right]\left[x_{j_{1}}, x_{j_{3}}\right], i_{1} \leq i_{2} \leq \ldots \leq i_{k}, j_{2} \leq j_{3}, \\
x_{i_{1}} x_{i_{2}} \ldots x_{i_{k}}\left[x_{j_{1}}, x_{j_{2}}\right] \ldots\left[x_{j_{2 l-1}}, x_{j_{2 l}}\right], i_{1} \leq i_{2} \leq \ldots \leq i_{k}, j_{1}<j_{2}<\ldots<j_{2 l}, l \geq 3, \\
x_{i_{1}} x_{i_{2}} \ldots x_{i_{k}}\left[x_{j_{1}}, x_{j_{2}}\right] \ldots\left[x_{j_{2 l-1}}, x_{\left.j_{2 l}\right]}\right]\left[x_{j_{2 l+1}}, x_{j_{2 l+2}}, x_{j_{2 l+3}}\right], i_{1} \leq i_{2} \leq \ldots \leq i_{k}, \\
j_{1}<j_{2}<\ldots<j_{2 l+3}, k \geq 0, l \geq 1
\end{gathered}
$$

em $Q_{4}=F\langle X\rangle / T^{(4)}$ forma uma base para esse espaço vetorial.

Demonstração. Faremos uso do Teorema 1.11. Seja $f \in B$ um polinômio próprio. Como $\left[x_{1}, x_{2}, x_{3}, x_{4}\right]$ e $\left[x_{1}, x_{2}, x_{3}\right]\left[x_{4}, x_{5}, x_{6}\right]$ pertencem a $T^{(4)}$ (Lema 1.32), não é difícil verificar que $f$ é uma combinação linear, módulo $T^{(4)}$, dos seguintes polinômios

$$
\begin{aligned}
b_{1} & =1 \\
b_{2} & =\left[x_{j_{1}}, x_{j_{2}}\right] \\
b_{3} & =\left[x_{j_{1}}, x_{j_{2}}, x_{j_{3}}\right] \\
b_{4} & =\left[x_{j_{1}}, x_{j_{2}}\right]\left[x_{j_{3}}, x_{j_{4}}\right] \\
b_{5} & =\left[x_{j_{1}}, x_{j_{2}}\right]\left[x_{j_{3}}, x_{j_{4}}\right] \ldots\left[x_{j_{2 l-1}}, x_{j_{2 l}}\right], l \geq 3, \\
b_{6} & =\left[x_{j_{1}}, x_{j_{2}}\right] \ldots\left[x_{j_{2 l-1}}, x_{j_{2 l}}\right]\left[x_{j_{2 l+1}}, x_{j_{2 l+2}}, x_{j_{2 l+3}}\right], l \geq 1
\end{aligned}
$$

Como $\left[x_{j_{1}}, x_{j_{2}}\right]=-\left[x_{j_{2}}, x_{j_{1}}\right]$ (lei anti-comutativa), segue que $b_{2}$ é múltiplo escalar do polinômio

$$
\left[x_{j_{1}}, x_{j_{2}}\right], j_{1}<j_{2} .
$$

Vejamos o polinômio $b_{3}$. Se $b_{3}$ tiver duas variáveis iguais, então segue da lei anticomutativa que ele é um múltiplo escalar de um polinômio da forma

$$
\left[x_{j_{1}}, x_{j_{2}}, x_{j_{2}}\right], j_{1} \neq j_{2}
$$

Se todas as variáveis de $b_{3}$ forem distintas, então segue da identidade de Jacobi e 
da lei anti-comutativa que $b_{3}$ é uma combinação linear de polinômios da forma

$$
\left[x_{j_{1}}, x_{j_{2}}, x_{j_{3}}\right], j_{1}>j_{2}<j_{3}
$$

Segue da relação $\left[\left[x_{1}, x_{2}\right],\left[x_{3}, x_{4}\right]\right]=\left[x_{1}, x_{2}, x_{3}, x_{4}\right]-\left[x_{1}, x_{2}, x_{4}, x_{3}\right] \in T^{(4)}$ que $b_{4}$ é uma combinação linear, módulo $T^{(4)}$, de polinômios da forma

$$
\begin{array}{ll}
{\left[x_{j_{1}}, x_{j_{2}}\right]\left[x_{j_{1}}, x_{j_{3}}\right],} & j_{2} \leq j_{3}, \\
{\left[x_{j_{1}}, x_{j_{2}}\right]\left[x_{j_{3}}, x_{j_{4}}\right],} & j_{1}<j_{2}, j_{3} \text { e } j_{3}<j_{4} .
\end{array}
$$

Pelo Corolário 1.33 (i) obtemos que $b_{5}$ é uma combinação linear, módulo $T^{(4)}$, dos polinômios

$$
\left[x_{j_{1}}, x_{j_{2}}\right]\left[x_{j_{3}}, x_{j_{4}}\right] \ldots\left[x_{j_{2 l-1}}, x_{j_{2 l}}\right], l \geq 3, j_{1}<j_{2}<\ldots<j_{2 l}
$$

Finalmente, segue do Lema 1.33 (ii) que $b_{6}$ é, módulo $T^{(4)}$, um múltiplo escalar de um polinômio da forma

$$
\left[x_{j_{1}}, x_{j_{2}}\right] \ldots\left[x_{j_{2 l-1}}, x_{j_{2 l}}\right]\left[x_{j_{2 l+1}}, x_{j_{2 l+2}}, x_{j_{2 l+3}}\right], l \geq 1, j_{1}<j_{2}<\ldots<j_{2 l+3} .
$$

Portanto a imagem dos polinômios (2-38)-(2-45) em $B /\left(B \cap T^{(4)}\right)$ gera esse espaço vetorial. Vamos mostrar que essa imagem forma um conjunto linearmente independente.

Seja $f$ uma combinação linear de polinômios em (2-38)-(2-45) com $f \equiv 0$ $\left(\bmod T^{(4)}\right)$. Escreva

$$
f=f_{1}+f_{2}
$$

onde $f_{1}$ é uma combinação linear de polinômios em (2-38),(2-39), (2-43) e (2-44) e $f_{2}$ é uma combinação linear dos polinômios em (2-40),(2-41), (2-42) e (2-45).

Como $f_{2} \in T^{(3)}, f \in T^{(4)}$ e $T^{(4)} \subseteq T^{(3)}$, segue que $f_{1} \equiv 0\left(\bmod T^{(3)}\right)$. Pela Proposição 1.21, a imagem dos polinômios de (2-38),(2-39), (2-43) e (2-44) em $F\langle X\rangle / T^{(3)}$ forma um conjunto linearmente independente. Desse modo, $f_{1} \equiv 0\left(\bmod T^{(3)}\right) \mathrm{im}$ plica $f_{1}=0$. Como $f=f_{1}+f_{2}$ e $f \equiv 0\left(\bmod T^{(4)}\right)$ temos

$$
f_{2} \equiv 0 \quad\left(\bmod T^{(4)}\right)
$$

Resta mostrar que os coeficientes correspondentes aos polinômios (2-40),(2-41), (2$42)$ e (2-45) que aparecem em $f_{2}$ devem ser nulos.

Afirmação 1: Os coeficientes dos polinômios das formas (2-40) e (2-42) (com 
$\left.j_{2}=j_{3}\right)$ que aparecem em $f_{2}$ são nulos. De fato, escreva

$$
f_{2}=\alpha\left[x_{j_{1}}, x_{j_{2}}, x_{j_{2}}\right]+\beta\left[x_{j_{1}}, x_{j_{2}}\right]\left[x_{j_{1}}, x_{j_{2}}\right]+\text { demais termos }
$$

onde $\alpha, \beta \in F$ e $j_{1}<j_{2}$. Agora considere o endomorfismo $\varphi_{j_{1} j_{2}}$ de $F\langle X\rangle$ tal que $\varphi_{j_{1} j_{2}}\left(x_{j_{s}}\right)=x_{j_{s}}$, se $s \in\{1,2\}$ e $\varphi_{j_{1} j_{2}}\left(x_{j_{s}}\right)=1$ se $s \notin\{1,2\}$. Segue de (2-46) e $(2-47)$ que

$$
\varphi_{j_{1} j_{2}}\left(f_{2}\right)=\alpha\left[x_{j_{1}}, x_{j_{2}}, x_{j_{2}}\right]+\beta\left[x_{j_{1}}, x_{j_{2}}\right]\left[x_{j_{1}}, x_{j_{2}}\right] \in T^{(4)} .
$$

Observe que $\alpha\left[x_{j_{1}}, x_{j_{2}}, x_{j_{2}}\right]$ e $\beta\left[x_{j_{1}}, x_{j_{2}}\right]\left[x_{j_{1}}, x_{j_{2}}\right]$ são componentes multi-homogêneas do polinômio $\varphi_{j_{1} j_{2}}\left(f_{2}\right)$. Como $T^{(4)}$ é multi-homogêneo (Lema 1.10), segue que os polinômios $\alpha\left[x_{j_{1}}, x_{j_{2}}, x_{j_{2}}\right]$ e $\beta\left[x_{j_{1}}, x_{j_{2}}\right]\left[x_{j_{1}}, x_{j_{2}}\right]$ pertencem a $T^{(4)}$. Como $\left[x_{j_{1}}, x_{j_{2}}, x_{j_{2}}\right] \notin$ $T^{(4)}$ (porque possui grau inferior a 4 ) temos $\alpha=0$. Também $\left[x_{j_{1}}, x_{j_{2}}\right]\left[x_{j_{1}}, x_{j_{2}}\right] \notin T^{(4)}$ (Lema 2.13). Assim $\beta=0$.

Afirmação 2: Os coeficientes dos polinômios das formas (2-41) e (2-42) (com $\left.j_{2}<j_{3}\right)$ que aparecem em $f_{3}$ são nulos. De fato, escreva

$$
f_{2}=\alpha\left[x_{j_{1}}, x_{j_{2}}, x_{j_{3}}\right]+\beta\left[x_{j_{3}}, x_{j_{2}}, x_{j_{1}}\right]+\gamma\left[x_{j_{1}}, x_{j_{2}}\right]\left[x_{j_{1}}, x_{j_{3}}\right]+\text { demais termos }
$$

onde $\alpha, \beta, \gamma \in F$ e $j_{2}<j_{1}, j_{3}$. Tome o endomorfismo $\varphi_{j_{1} j_{2} j_{3}}$ de $F\langle X\rangle$ tal que $\varphi_{j_{1} j_{2} j_{3}}\left(x_{j_{s}}\right)=x_{j_{s}}$, se $s \in\{1,2,3\}$ e $\varphi_{j_{1} j_{2} j_{3}}\left(x_{j_{s}}\right)=1$ se $s \notin\{1,2,3\}$. Por $(2-46)$ e $(2-$ 48), temos

$$
\varphi_{j_{1} j_{2} j_{3}}\left(f_{2}\right)=\alpha\left[x_{j_{1}}, x_{j_{2}}, x_{j_{3}}\right]+\beta\left[x_{j_{3}}, x_{j_{2}}, x_{j_{1}}\right]+\gamma\left[x_{j_{1}}, x_{j_{2}}\right]\left[x_{j_{1}}, x_{j_{3}}\right] \in T^{(4)}
$$

Como $T^{(4)}$ é multi-homogêneo, segue que as componentes multi-homogêneas $h_{1}=$ $\alpha\left[x_{j_{1}}, x_{j_{2}}, x_{j_{3}}\right]+\beta\left[x_{j_{3}}, x_{j_{2}}, x_{j_{1}}\right]$ e $h_{2}=\gamma\left[x_{j_{1}}, x_{j_{2}}\right]\left[x_{j_{1}}, x_{j_{3}}\right]$ de $\varphi_{j_{1} j_{2} j_{3}}(f)$ pertencem a $T^{(4)}$.

Portanto $\alpha=\beta=0$, caso contrário $\operatorname{deg} h_{1}=3$ e teríamos $h_{1} \notin T^{(4)}$, uma contradição. Como $\left[x_{j_{1}}, x_{j_{2}}\right]\left[x_{j_{1}}, x_{j_{3}}\right] \notin T^{(4)}$, temos $\gamma=0$.

Afirmação 3: Os coeficientes dos polinômios da forma (2-45) que aparecem em $f_{2}$ são nulos. Isso segue diretamente do Teorema 2.11.

Segue das Afirmações 1-3 que a imagem dos polinômios (2-38)-(2-45) em $B /\left(B \cap T^{(4)}\right)$ forma uma base para esse espaço vetorial. Finalmemente, segue do Teorema 1.11 que a imagem dos polinômios (i)-(viii) em $Q_{4}=F\langle X\rangle / T^{(4)}$ forma uma base para esse espaço vetorial. 


\subsubsection{Uma base para o espaço vetorial $T^{(3)} / T^{(4)}$}

Vejamos mais algumas propriedades do polinômio

$$
\omega\left(x_{1}, x_{2}, x_{3}, x_{4}\right)=\left[x_{1}, x_{2}\right]\left[x_{3}, x_{4}\right]+\left[x_{1}, x_{3}\right]\left[x_{2}, x_{4}\right]
$$

Observe que $\omega$ é simétrico nas variáveis $x_{2}$ e $x_{3}$, isto é

$$
\omega\left(x_{1}, x_{2}, x_{3}, x_{4}\right)=\omega\left(x_{1}, x_{3}, x_{2}, x_{4}\right) .
$$

O polinômio $\omega$ safisfaz também duas "identidades de Jacobi" (veja [44] e [45]):

$$
\begin{aligned}
& \omega\left(x_{1}, x_{2}, x_{3}, x_{4}\right)+\omega\left(x_{2}, x_{3}, x_{1}, x_{4}\right)+\omega\left(x_{3}, x_{1}, x_{2}, x_{4}\right)=0, \\
& \omega\left(x_{1}, x_{2}, x_{3}, x_{4}\right)+\omega\left(x_{1}, x_{3}, x_{4}, x_{2}\right)+\omega\left(x_{1}, x_{4}, x_{2}, x_{3}\right)=0 .
\end{aligned}
$$

A verificação de (2-50) e (2-51) são diretas, basta usar a definição de $\boldsymbol{\omega}$ e fazer os cancelamentos que ocorrerem.

As duas relações seguintes (veja [44]) serão usadas no texto :

$$
\begin{array}{ll}
\omega\left(x_{1}, x_{2}, x_{3}, x_{4}\right) \equiv \omega\left(x_{3}, x_{4}, x_{1}, x_{2}\right) & \left(\bmod T^{(4)}\right), \\
\omega\left(x_{1}, x_{2}, x_{3}, x_{4}\right) \equiv \omega\left(x_{2}, x_{1}, x_{4}, x_{3}\right) & \left(\bmod T^{(4)}\right) .
\end{array}
$$

Vamos prová-las. Observando que $\left[\left[x_{1}, x_{2}\right],\left[x_{3}, x_{4}\right]\right]=\left[x_{1}, x_{2}, x_{3}, x_{4}\right]-\left[x_{1}, x_{2}, x_{4}, x_{3}\right] \in$ $T^{(4)}$, temos

$$
\begin{aligned}
\omega\left(x_{1}, x_{2}, x_{3}, x_{4}\right) & =\left[x_{1}, x_{2}\right]\left[x_{3}, x_{4}\right]+\left[x_{1}, x_{3}\right]\left[x_{2}, x_{4}\right] \\
& =\left[x_{3}, x_{4}\right]\left[x_{1}, x_{2}\right]+\left[\left[x_{1}, x_{2}\right],\left[x_{3}, x_{4}\right]\right]+\left[x_{1}, x_{3}\right]\left[x_{2}, x_{4}\right] \\
& \equiv\left[x_{3}, x_{4}\right]\left[x_{1}, x_{2}\right]+\left[x_{3}, x_{1}\right]\left[x_{4}, x_{2}\right] \quad\left(\bmod T^{(4)}\right) \\
& \equiv \omega\left(x_{3}, x_{4}, x_{1}, x_{2}\right) \quad\left(\bmod T^{(4)}\right),
\end{aligned}
$$

que é a relação (2-52). Também

$$
\begin{aligned}
\omega\left(x_{1}, x_{2}, x_{3}, x_{4}\right) & =\left[x_{1}, x_{2}\right]\left[x_{3}, x_{4}\right]+\left[x_{1}, x_{3}\right]\left[x_{2}, x_{4}\right] \\
& =\left[x_{2}, x_{4}\right]\left[x_{1}, x_{3}\right]+\left[\left[x_{1}, x_{3}\right],\left[x_{2}, x_{4}\right]\right]+\left[x_{1}, x_{2}\right]\left[x_{3}, x_{4}\right] \\
& \equiv\left[x_{2}, x_{4}\right]\left[x_{1}, x_{3}\right]+\left[x_{2}, x_{1}\right]\left[x_{4}, x_{3}\right] \quad\left(\bmod T^{(4)}\right) \\
& \equiv \omega\left(x_{2}, x_{1}, x_{4}, x_{3}\right) \quad\left(\bmod T^{(4)}\right),
\end{aligned}
$$

que é a relação (2-53). 
Defina o seguinte subespaço vetorial de $F\langle X\rangle$ :

$$
M=\operatorname{Span}\left\{\omega\left(x_{j_{1}}, x_{j_{2}}, x_{j_{3}}, x_{j_{4}}\right) \mid\left\{j_{1}, j_{2}, j_{3}, j_{4}\right\} \subseteq \mathbb{N}\right\}
$$

Quando $F$ possui característica 0 , uma base para o espaço vetorial $(M+$ $\left.T^{(4)}\right) / T^{(4)}$ foi encontrada por I. B. Volichenko [44] (veja também [45]). A seguir, exibimos uma base para o espaço vetorial $\left(M+T^{(4)}\right) / T^{(4)}$ quando $F$ possui característica 3.

Proposição 2.15. Seja F um corpo de característica 3. Então o espaço vetorial $\left(M+T^{(4)}\right) / T^{(4)}$ possui uma base formada pelos elementos

$$
\begin{aligned}
& \omega\left(x_{j_{1}}, x_{j_{2}}, x_{j_{3}}, x_{j_{4}}\right)+T^{(4)}, \omega\left(x_{j_{1}}, x_{j_{2}}, x_{j_{4}}, x_{j_{3}}\right)+T^{(4)}, j_{1}<j_{2}<j_{3}<j_{4}, \\
& \omega\left(x_{j_{1}}, x_{j_{1}}, x_{j_{2}}, x_{j_{3}}\right)+T^{(4)}, j_{2}<j_{3} .
\end{aligned}
$$

Demonstração. Vamos mostrar que os elementos (2-54)-(2-55) geram o espaço vetorial $\left(M+T^{(4)}\right) / T^{(4)}$. Observe primeiramente que se $\omega\left(x_{j_{1}}, x_{j_{2}}, x_{j_{3}}, x_{j_{4}}\right)$ tiver mais de duas variáveis iguais, então $\omega\left(x_{j_{1}}, x_{j_{2}}, x_{j_{3}}, x_{j_{4}}\right)$ se anula. Assim podemos assumir que cada gerador $\omega\left(x_{j_{1}}, x_{j_{2}}, x_{j_{3}}, x_{j_{4}}\right)$ de $M$ possui no máximo duas variáveis iguais.

Fixemos $J=\left\{l_{1}, l_{2}, l_{3}, l_{4}\right\} \subseteq \mathbb{N}$ e seja $J=\left\{j_{1}, j_{2}\right\} \cup\left\{j_{3}, j_{4}\right\}$ uma união disjunta qualquer. Afirmamos que todo elemento $\omega\left(x_{l_{1}}, x_{l_{2}}, x_{l_{3}}, x_{l_{4}}\right)+T^{(4)}$ é uma combinação linear de elementos da forma

$$
\omega\left(x_{j_{1}}, x_{j_{2}}, x_{l_{3}}, x_{l_{4}}\right)+T^{(4)}, \omega\left(x_{j_{1}}, x_{j_{2}}, x_{l_{4}}, x_{l_{3}}\right)+T^{(4)}
$$

De fato, aplicando (2-53) duas vezes temos

$$
\omega\left(x_{l_{1}}, x_{l_{2}}, x_{l_{3}}, x_{j_{1}}\right) \equiv \omega\left(x_{l_{2}}, x_{l_{1}}, x_{j_{1}}, x_{l_{3}}\right) \equiv \omega\left(x_{l_{2}}, x_{j_{1}}, x_{l_{1}}, x_{l_{3}}\right) \equiv \omega\left(x_{j_{1}}, x_{l_{2}}, x_{l_{3}}, x_{l_{1}}\right) \quad\left(\bmod T^{(4)}\right) .
$$

Assim, todo elemento $\omega\left(x_{l_{1}}, x_{l_{2}}, x_{l_{3}}, x_{l_{4}}\right)+T^{(4)}$ pode ser escrito da forma

$$
\omega\left(x_{j_{1}}, x_{l_{2}}, x_{l_{3}}, x_{l_{4}}\right)+T^{(4)} .
$$

Por (2-51), temos

$$
\begin{aligned}
\omega\left(x_{j_{1}}, x_{l_{2}}, x_{l_{3}}, x_{j_{2}}\right) & =-\omega\left(x_{j_{1}}, x_{l_{3}}, x_{j_{2}}, x_{l_{2}}\right)-\omega\left(x_{j_{1}}, x_{j_{2}}, x_{l_{2}}, x_{l_{3}}\right) \\
& =-\omega\left(x_{j_{1}}, x_{j_{2}}, x_{l_{3}}, x_{l_{2}}\right)-\omega\left(x_{j_{1}}, x_{j_{2}}, x_{l_{2}}, x_{l_{3}}\right) .
\end{aligned}
$$

Como $\omega\left(x_{j_{1}}, x_{l_{2}}, x_{j_{2}}, x_{j_{4}}\right)=\omega\left(x_{j_{1}}, x_{j_{2}}, x_{l_{2}}, x_{j_{4}}\right)$, segue de (2-58) que todo elemento da forma $\omega\left(x_{l_{1}}, x_{l_{2}}, x_{l_{3}}, x_{l_{4}}\right)+T^{(4)}$ pode ser escrito como uma combinação linear dos elementos (2-56). Isso prova a afirmação feita. 
Seja $\omega\left(x_{l_{1}}, x_{l_{2}}, x_{l_{3}}, x_{l_{4}}\right)$ um gerador qualquer do espaço vetorial $M$ e suponha que todas as suas variáveis sejam distintas. Assim $\left\{l_{1}, l_{2}, l_{3}, l_{4}\right\}=\left\{j_{1}, j_{2}, j_{3}, j_{4}\right\}$ com $j_{1}<j_{2}<j_{3}<j_{4}$. Segue da afirmação provada acima que $\omega\left(x_{l_{1}}, x_{l_{2}}, x_{l_{3}}, x_{l_{4}}\right)+T^{(4)}$ pode ser escrito como uma combinação linear dos elementos $\omega\left(x_{j_{1}}, x_{j_{2}}, x_{j_{3}}, x_{j_{4}}\right)+T^{(4)}$ e $\omega\left(x_{j_{1}}, x_{j_{2}}, x_{j_{4}}, x_{j_{3}}\right)+T^{(4)} \operatorname{com} j_{1}<j_{2}<j_{3}<j_{4}$ (que são da forma 2-54).

Agora, suponha que o polinômio $\omega\left(x_{l_{1}}, x_{l_{2}}, x_{l_{3}}, x_{l_{4}}\right)$ possua duas variáveis repetidas. Assim $\left\{l_{1}, l_{2}, l_{3}, l_{4}\right\}=\left\{j_{1}, j_{2}, j_{3}\right\}$ com $j_{2}<j_{3}$. Segue novamente da afirmação provada que $\omega\left(x_{l_{1}}, x_{l_{2}}, x_{l_{3}}, x_{l_{4}}\right)+T^{(4)}$ é uma combinação linear dos elementos $\omega\left(x_{j_{1}}, x_{j_{1}}, x_{j_{2}}, x_{j_{3}}\right)+T^{(4)}$ e $\omega\left(x_{j_{1}}, x_{j_{1}}, x_{j_{3}}, x_{j_{2}}\right)+T^{(4)}\left(j_{2}<j_{3}\right)$. Como $\left[\left[x_{1}, x_{2}\right],\left[x_{3}, x_{4}\right]\right]=$ $\left[x_{1}, x_{2}, x_{3}, x_{4}\right]-\left[x_{1}, x_{2}, x_{4}, x_{3}\right] \in T^{(4)}$, temos

$$
\begin{aligned}
\omega\left(x_{j_{1}}, x_{j_{1}}, x_{j_{3}}, x_{j_{2}}\right) & =\left[x_{j_{1}}, x_{j_{3}}\right]\left[x_{j_{1}}, x_{j_{2}}\right] \\
& \equiv\left[x_{j_{1}}, x_{j_{2}}\right]\left[x_{j_{1}}, x_{j_{3}}\right]\left(\bmod T^{(4)}\right) \\
& =\omega\left(x_{j_{1}}, x_{j_{1}}, x_{j_{2}}, x_{j_{3}}\right) .
\end{aligned}
$$

Assim $\omega\left(x_{l_{1}}, x_{l_{2}}, x_{l_{3}}, x_{l_{4}}\right)+T^{(4)}$ é um múltiplo escalar do elemento $\omega\left(x_{j_{1}}, x_{j_{1}}, x_{j_{2}}, x_{j_{3}}\right)+$ $T^{(4)}$ com $j_{2}<j_{3}$ (que é da forma (2-55)). Mostramos assim que os elementos (2-54)(2-55) geram o espaço vetorial $\left(M+T^{(4)}\right) / T^{(4)}$.

Vamos mostrar que esses elementos são linearmente independentes. De fato, seja

$$
\sum_{J} \alpha_{J} \omega\left(j_{1}, j_{2}, j_{3}, j_{4}\right)+T^{(4)}=T^{(4)}
$$

onde cada $\alpha_{J} \in F, J=\left(j_{1}, j_{2}, j_{3}, j_{4}\right)$ e os $\omega\left(j_{1}, j_{2}, j_{3}, j_{4}\right)+T^{(4)}$ são elementos de (2-54)-(2-55). Observe que $J$ é de uma das seguintes formas: $J_{1}=\left(j_{1}, j_{2}, j_{3}, j_{4}\right)$, $J_{2}=\left(j_{1}, j_{2}, j_{4}, j_{3}\right)$ com $j_{1}<j_{2}<j_{3}<j_{4}$ ou $J_{3}=\left(j_{1}, j_{1}, j_{2}, j_{3}\right)$ com $j_{2}<j_{3}$. Como

$$
\begin{aligned}
& \alpha_{J_{1}} \omega\left(x_{j_{1}}, x_{j_{2}}, x_{j_{3}}, x_{j_{4}}\right)+\alpha_{J_{2}} \omega\left(x_{j_{1}}, x_{j_{2}}, x_{j_{4}}, x_{j_{3}}\right)= \\
& =\left(\alpha_{J_{1}}-\alpha_{J_{2}}\right)\left[x_{j_{1}}, x_{j_{2}}\right]\left[x_{j_{3}}, x_{j_{4}}\right]+\alpha_{J_{1}}\left[x_{j_{1}}, x_{j_{3}}\right]\left[x_{j_{2}}, x_{j_{4}}\right]+\alpha_{J_{2}}\left[x_{j_{1}}, x_{j_{4}}\right]\left[x_{j_{2}}, x_{j_{3}}\right]
\end{aligned}
$$

e

$$
\omega\left(x_{j_{1}}, x_{j_{1}}, x_{j_{2}}, x_{j_{3}}\right)=\left[x_{j_{1}}, x_{j_{2}}\right]\left[x_{j_{1}}, x_{j_{3}}\right]
$$

podemos escrever a igualdade (2-59) da seguinte forma

$$
\begin{aligned}
& \sum\left(\alpha_{J_{1}}-\alpha_{J_{2}}\right)\left[x_{j_{1}}, x_{j_{2}}\right]\left[x_{j_{3}}, x_{j_{4}}\right]+\sum \alpha_{J_{1}}\left[x_{j_{1}}, x_{j_{3}}\right]\left[x_{j_{2}}, x_{j_{4}}\right]+\sum \alpha_{J_{2}}\left[x_{j_{1}}, x_{j_{4}}\right]\left[x_{j_{2}}, x_{j_{3}}\right] \\
& +\sum \alpha_{J_{3}}\left[x_{j_{1}}, x_{j_{2}}\right]\left[x_{j_{1}}, x_{j_{3}}\right]+T^{(4)}=T^{(4)}
\end{aligned}
$$

Como o lado esquerdo da igualdade (2-61) é uma combinação linear de elementos da base de $F\langle X\rangle / T^{(4)}$ (Proposição 2.14), segue que todos os $\alpha_{J_{1}}, \alpha_{J_{2}}$ e $\alpha_{J_{3}}$ são nulos. 
Portanto todos os $\alpha_{J}$ da igualdade (2-59) são nulos. Isso mostra que os elementos (2-54)-(2-55) são linearmente independentes e portanto, formam uma base para o espaço vetorial $\left(M+T^{(4)}\right) / T^{(4)}$.

Lema 2.16. Seja $F$ um corpo qualquer. Então $x_{1}\left[x_{2}, x_{3}, x_{3}\right]+T^{(4)} e$ $x_{1} \omega\left(x_{2}, x_{3}, x_{4}, x_{5}\right)+T^{(4)}$ são centrais na álgebra $Q_{4}=F\langle X\rangle / T^{(4)}$.

Demonstração. De fato

$$
\left[x_{1}\left[x_{2}, x_{3}, x_{3}\right], x_{4}\right]=x_{1}\left[x_{2}, x_{3}, x_{3}, x_{4}\right]+\left[x_{1}, x_{4}\right]\left[x_{2}, x_{3}, x_{3}\right]
$$

É claro que $x_{1}\left[x_{2}, x_{3}, x_{3}, x_{4}\right]$ pertence a $T^{(4)}$. Como $\left[x_{1}, x_{4}\right]\left[x_{2}, x_{3}, x_{3}\right]$ também pertence a $T^{(4)}$ (Corolário $1.33\left(\right.$ ii)), segue que $\left[x_{1}\left[x_{2}, x_{3}, x_{3}\right], x_{4}\right]$ pertence a $T^{(4)}$, isto é $x_{1}\left[x_{2}, x_{3}, x_{3}\right]+T^{(4)}$ é central em $Q_{4}$.

Usando as igualdades $[a b, c]=a[b, c]+[a, c] b$ e $a b=b a+[a, b]$, obtemos

$$
\begin{aligned}
{\left[\omega\left(x_{1}, x_{2}, x_{3}, x_{4}\right), x_{5}\right] } & =\left[\left[x_{1}, x_{2}\right]\left[x_{3}, x_{4}\right], x_{5}\right]+\left[\left[x_{1}, x_{3}\right]\left[x_{2}, x_{4}\right], x_{5}\right] \\
& =\left[x_{1}, x_{2}\right]\left[x_{3}, x_{4}, x_{5}\right]+\left[x_{1}, x_{2}, x_{5}\right]\left[x_{3}, x_{4}\right]+ \\
& +\left[x_{1}, x_{3}\right]\left[x_{2}, x_{4}, x_{5}\right]+\left[x_{1}, x_{3}, x_{5}\right]\left[x_{2}, x_{4}\right] \\
& \equiv\left[x_{3}, x_{4}, x_{5}\right]\left[x_{1}, x_{2}\right]+\left[x_{1}, x_{2}, x_{5}\right]\left[x_{3}, x_{4}\right]+ \\
& +\left[x_{2}, x_{4}, x_{5}\right]\left[x_{1}, x_{3}\right]+\left[x_{1}, x_{3}, x_{5}\right]\left[x_{2}, x_{4}\right] . \quad\left(\bmod T^{(4)}\right)
\end{aligned}
$$

Pelo Lema 1.32, os polinômios $\left[x_{3}, x_{4}, x_{5}\right]\left[x_{1}, x_{2}\right]+\left[x_{2}, x_{4}, x_{5}\right]\left[x_{1}, x_{3}\right]$ e $\left[x_{1}, x_{2}, x_{5}\right]\left[x_{3}, x_{4}\right]+$ $\left[x_{1}, x_{3}, x_{5}\right]\left[x_{2}, x_{4}\right]$ pertencem a $T^{(4)}$. Logo

$$
\left[\omega\left(x_{1}, x_{2}, x_{3}, x_{4}\right), x_{5}\right] \in T^{(4)}
$$

Agora

$$
\left[x_{1} \omega\left(x_{2}, x_{3}, x_{4}, x_{5}\right), x_{6}\right]=x_{1}\left[\omega\left(x_{2}, x_{3}, x_{4}, x_{5}\right), x_{6}\right]+\left[x_{1}, x_{6}\right] \omega\left(x_{2}, x_{3}, x_{4}, x_{5}\right) .
$$

Como $\left[x_{1}, x_{6}\right] \omega\left(x_{2}, x_{3}, x_{4}, x_{5}\right) \in T^{(4)}$ (Lema 1.32), segue de (2-62) e (2-63) que $\left[x_{1} \omega\left(x_{2}, x_{3}, x_{4}, x_{5}\right), x_{6}\right]$ pertence a $T^{(4)}$, isto é, $x_{1} \omega\left(x_{2}, x_{3}, x_{4}, x_{5}\right)+T^{(4)}$ é central em $Q_{4}$.

Quando $F$ possui característica 0, Volichenko [44] encontrou uma base para o espaço vetorial $T^{(3)} / T^{(4)}$. A seguir exibimos uma base para o espaço vetorial $T^{(3)} / T^{(4)}$ quando $F$ possui característica 3. 
Proposição 2.17. Seja F um corpo de característica 3. Então a imagem dos polinômios

$$
\begin{gathered}
x_{i_{1}} x_{i_{2}} \ldots x_{i_{k}}\left[x_{j_{1}}, x_{j_{2}}, x_{j_{2}}\right], i_{1} \leq i_{2} \leq \ldots \leq i_{k}, j_{1} \neq j_{2}, \\
x_{i_{1}} x_{i_{2}} \ldots x_{i_{k}}\left[x_{j_{1}}, x_{j_{2}}, x_{j_{3}}\right], i_{1} \leq i_{2} \leq \ldots \leq i_{k}, j_{1}>j_{2}<j_{3}, \\
x_{i_{1}} x_{i_{2}} \ldots x_{i_{k}} \omega\left(x_{j_{1}}, x_{j_{2}}, x_{j_{3}}, x_{j_{4}}\right) ; x_{i_{1}} x_{i_{2}} \ldots x_{i_{k}} \omega\left(x_{j_{1}}, x_{j_{2}}, x_{j_{4}}, x_{j_{3}}\right), \\
i_{1} \leq i_{2} \leq \ldots \leq i_{k}, j_{1}<j_{2}<j_{3}<j_{4}, \\
x_{i_{1}} x_{i_{2}} \ldots x_{i_{k}} \omega\left(x_{j_{1}}, x_{j_{1}}, x_{j_{2}}, x_{j_{3}}\right), \\
i_{1} \leq i_{2} \leq \ldots \leq i_{k}, j_{2}<j_{3}, \\
x_{i_{1}} x_{i_{2}} \ldots x_{i_{k}}\left[x_{j_{1}}, x_{j_{2}}\right] \ldots\left[x_{j_{2 l-1}}, x_{l_{2 l}}\right]\left[x_{j_{2 l+1}}, x_{j_{2 l+2}}, x_{j_{2 l+3}}\right] \\
i_{1} \leq i_{2} \leq \ldots \leq i_{k}, j_{1}<j_{2}<\ldots<j_{2 l+3}, k \geq 0, l \geq 1
\end{gathered}
$$

em $Q_{4}=F\langle X\rangle / T^{(4)}$ forma uma base para o espaço vetorial $T^{(3)} / T^{(4)}$.

Demonstração. Vamos mostrar primeiramente que a imagem dos polinômios (264)-(2-68) em $Q_{4}=F\langle X\rangle / T^{(4)}$ gera o espaço vetorial $T^{(3)} / T^{(4)}$. Como $\left[x_{1}, x_{2}, x_{3}\right]$ e $\omega\left(x_{1}, x_{2}, x_{3}, x_{4}\right)$ pertencem a $T^{(3)}$ (Lema $1.18(\mathrm{i})$ ), segue que os polinômios (264)-(2-68) pertencem a $T^{(3)}$. Assim a imagem dos polinômios (2-64)-(2-68) em $Q_{4}=F\langle X\rangle / T^{(4)}$ está contida em $T^{(3)} / T^{(4)}$.

Pela Proposição 1.20, $T^{(3)}$ é gerado, como um ideal bilateral de $F\langle X\rangle$, pelos polinômios $\left[x_{i_{1}}, x_{i_{2}}, x_{i_{3}}\right]\left(x_{i_{s}} \in X\right)$ e $\omega\left(x_{j_{1}}, x_{j_{2}}, x_{j_{3}}, x_{j_{4}}\right)\left(x_{j_{s}} \in X\right)$. Assim todo polinômio $f \in T^{(3)}$ pode ser escrito como uma combinação linear de polinômios da forma

$$
\begin{aligned}
& x_{i_{1}} \ldots x_{i_{r}}\left[x_{j_{1}}, x_{j_{2}}, x_{j_{3}}\right] x_{i_{r+1}} \ldots x_{i_{k}} \\
& x_{i_{1}} \ldots x_{i_{r}} \omega\left(x_{j_{1}}, x_{j_{2}}, x_{j_{3}}, x_{j_{4}}\right) x_{i_{r+1}} \ldots x_{i_{k}} .
\end{aligned}
$$

Vamos mostrar primeiramente que os polinômios da forma (2-69) podem ser escritos, módulo $T^{(4)}$, como uma combinação linear dos polinômios (2-64)-(268). Como $\left[x_{j_{1}}, x_{j_{2}}, x_{j_{3}}\right]+T^{(4)}$ é central de $Q_{4}$, segue que cada polinômio da forma (2-69) pode ser escrito, módulo $T^{(4)}$, como

$$
x_{i_{1}} x_{i_{2}} \ldots x_{i_{k}}\left[x_{j_{1}}, x_{j_{2}}, x_{j_{3}}\right]
$$

Usando a igualdade $a b=b a+[a, b]$ para ordenar as variáveis $x_{i_{1}}, x_{i_{2}}, \ldots, x_{i_{k}}$ do polinômio (2-71), bem como a relação $\left[x_{1}, x_{2}, x_{3}\right]\left[x_{4}, x_{5}, x_{6}\right] \in T^{(4)}$ (Lema 1.32), podemos verificar que cada polinômio da forma (2-71) pode ser escrito, módulo 
$T^{(4)}$, como uma combinação linear de polinômios da forma

$$
\begin{aligned}
& x_{i_{1}} x_{i_{2}} \ldots x_{i_{k}}\left[x_{j_{1}}, x_{j_{2}}, x_{j_{3}}\right], i_{1} \leq \ldots \leq i_{k} \\
& x_{i_{1}} x_{i_{2}} \ldots x_{i_{k}}\left[x_{j_{1}}, x_{j_{2}}\right] \ldots\left[x_{j_{2 l-1}}, x_{l_{2 l}}\right]\left[x_{j_{2 l+1}}, x_{j_{2 l+2}}, x_{j_{2 l+3}}\right], i_{1} \leq i_{2} \leq \ldots \leq i_{k} .
\end{aligned}
$$

Vejamos o polinômio (2-72). Se o comutador triplo $\left[x_{j_{1}}, x_{j_{2}}, x_{j_{3}}\right]$ do polinômio (2-72) tiver duas variáveis iguais, então é fácil ver que o polinômio (2-72) é um múltiplo escalar de algum polinômio da forma (2-64). Se o comutador triplo $\left[x_{j_{1}}, x_{j_{2}}, x_{j_{3}}\right]$ do polinômio (2-72) tiver todas as suas variáveis distintas, então segue da identidade de Jacobi e da lei anti-comutativa que o polinômio (2-72) pode ser escrito como uma combinação linear de polinômios da forma (2-65).

Vejamos o polinômio (2-73). Segue do Corolário 1.33 (ii) que módulo $T^{(4)}$, todas as variáveis dentro dos comutadores do polinômio (2-73) podem ser ordenadas, isto é, módulo $T^{(4)}$, cada polinômio (2-73) é um múltiplo escalar de algum polinômio da forma (2-68). Isso conclui a afirmação de que todo polinômio da forma (2-69) pode ser escrito, módulo $T^{(4)}$, como uma combinação linear dos polinômios (2-64)-(2-68).

Vamos mostrar agora que todo polinômio da forma (2-70) pode ser escrito, módulo $T^{(4)}$, como uma combinação linear dos polinômios (2-66)-(2-67). Como $\omega\left(x_{1}, x_{2}, x_{3}, x_{4}\right)+T^{(4)}$ é central em $Q_{4}$ (Lema 2.16), segue que o polinômio (2-70) pode ser escrito, módulo $T^{(4)}$, como

$$
x_{i_{1}} x_{i_{2}} \ldots x_{i_{k}} \omega\left(x_{j_{1}}, x_{j_{2}}, x_{j_{3}}, x_{j_{4}}\right)
$$

Usando a igualdade $a b=b a+[a, b]$ e a relação $\left[x_{5}, x_{6}\right] \omega\left(x_{1}, x_{2}, x_{3}, x_{4}\right) \in T^{(4)}$ (Lema 1.32), vemos que as variáveis $x_{i_{1}}, x_{i_{2}}, \ldots, x_{i_{k}}$ do polinômio (2-74) podem ser ordenadas, isto é, módulo $T^{(4)}$, cada polinômio da forma (2-74) pode ser escrito como

$$
x_{i_{1}} x_{i_{2}} \ldots x_{i_{k}} \omega\left(x_{j_{1}}, x_{j_{2}}, x_{j_{3}}, x_{j_{4}}\right), i_{1}<i_{2}<\ldots<i_{k} .
$$

Finalmente, segue do Lema 2.15 que cada polinômio da forma (2-75) pode ser escrito, módulo $T^{(4)}$, como uma combinação linear dos polinômios (2-66)-(2-67). Isso conclui a afirmação de que todo polinômio da forma (2-70) pode ser escrito, módulo $T^{(4)}$, como uma combinação linear dos polinômios de (2-66)-(2-67).

Mostramos assim que todo polinômio $f \in T^{(3)}$ pode ser escrito, módulo $T^{(4)}$, como uma combinação linear dos polinômios (2-64)-(2-68), isto é, a imagem dos polinômios (2-64)-(2-68) em $Q_{4}=F\langle X\rangle / T^{(4)}$ gera $T^{(3)} / T^{(4)}$.

Agora vamos mostrar que essa imagem forma um conjunto linearmente 
independente. De fato, seja

$$
\sum_{i} \delta_{i} f_{i} \equiv 0 \quad\left(\bmod T^{(4)}\right)
$$

onde $\delta_{i} \in F$ e os $g_{i}$ são polinômios distintos de (2-64)-(2-68). Como cada polinômio da forma $\alpha x_{i_{1}} \ldots x_{i_{k}} \omega\left(x_{j_{1}}, x_{j_{2}}, x_{j_{3}}, x_{j_{4}}\right)+\beta x_{i_{1}} \ldots x_{i_{k}} \omega\left(x_{j_{1}}, x_{j_{2}}, x_{j_{4}}, x_{j_{3}}\right)\left(i_{1} \leq \ldots \leq\right.$ $\left.i_{k}, j_{1}<j_{2}<j_{3}<j_{4}\right)$ pode ser escrito como $(\alpha-\beta) x_{i_{1}} \ldots x_{i_{k}}\left[x_{j_{1}}, x_{j_{2}}\right]\left[x_{j_{3}}, x_{j_{4}}\right]+$ $\alpha x_{i_{1}} \ldots x_{i_{k}}\left[x_{j_{1}}, x_{j_{3}}\right]\left[x_{j_{2}}, x_{j_{4}}\right]+\beta x_{i_{1}} \ldots x_{i_{k}}\left[x_{j_{1}}, x_{j_{4}}\right]\left[x_{j_{2}}, x_{j_{3}}\right]$, segue que a expressão (2-76) pode ser escrita como

$$
\sum_{i} \gamma_{i} g_{i}+\sum_{j}\left(\alpha_{j}-\beta_{j}\right) h_{j}+\sum_{j} \alpha_{j} u_{j}+\sum_{j} \beta_{j} v_{j} \equiv 0 \quad\left(\bmod T^{(4)}\right)
$$

onde os $g_{i}$ são polinômios distintos de (2-32), (2-33),(2-34) e (2-37) (da Proposição 2.14) e $h_{j}, u_{j}, v_{j}$ são polinômios distintos de (2-35) (também da Proposição 2.14).

Como todos os polinômio $g_{i}, h_{j}, u_{j}, v_{j}$ também são distintos e a imagem deles em $F\langle X\rangle / T^{(4)}$ são parte da base de $F\langle X\rangle / T^{(4)}$ (Lema 2.14), segue de (2-77) que todos os coeficientes $\gamma_{i}, \alpha_{j}, \beta_{j}$ são nulos. Portanto cada $\delta_{i}$ em 2-76 também é nulo e isso mostra que a imagem dos polinômios (2-64)-(2-68) em $Q_{4}=F\langle X\rangle / T^{(4)}$ forma um conjunto linearmente independente.

\subsubsection{Os polinômios centrais da álgebra $C\left(Q_{4}\right) \cap T^{(3)}$}

Começaremos com uma descrição dos polinômios de $T^{(3)}$ que são homogêneos de grau 1 em $x_{1}$.

Lema 2.18. Sejam $F$ um corpo de característica 3 e $f=f\left(x_{1}, \ldots, x_{n}\right) \in T^{(3)}$ um polinômio homogêneo de grau 1 em $x_{1}$. Então existem polinômios $g_{1}=g_{1}\left(x_{2}, \ldots, x_{n}\right) \in$ $F\langle X\rangle$ (que não depende de $\left.x_{1}\right), g_{2} \in\left\langle\left[x_{1}, x_{2}, x_{3}\right]\right\rangle^{T S}, g_{3} \in\left\langle\omega\left(x_{1}, x_{2}, x_{3}, x_{4}\right)\right\rangle^{T S}$ e $g_{4} \in T^{(4)}$ tais que $f=x_{1} g_{1}+g_{2}+g_{3}+g_{4}$.

Demonstração. Seja $f=f\left(x_{1}, \ldots, x_{n}\right) \in T^{(3)}$ um polinômio homogêneo de grau 1 em $x_{1}$. Segue da Proposição 2.17 que $f$ é uma combinação linear, módulo $T^{(4)}$, de polinômios das seguintes formas:

(i) $x_{i_{1}} x_{i_{2}} \ldots x_{i_{k}}\left[x_{j_{1}}, x_{j_{2}}, x_{j_{3}}\right], i_{1} \leq i_{2} \leq \ldots \leq i_{k}, j_{1}>j_{2} \leq j_{3}$,

(ii) $x_{i_{1}} x_{i_{2}} \ldots x_{i_{k}} \omega\left(x_{j_{1}}, x_{j_{2}}, x_{j_{3}}, x_{j_{4}}\right) ; x_{i_{1}} x_{i_{2}} \ldots x_{i_{k}} \omega\left(x_{j_{1}}, x_{j_{2}}, x_{j_{4}}, x_{j_{3}}\right)$,

$i_{1} \leq i_{2} \leq \ldots \leq i_{k}, j_{1}<j_{2}<j_{3}<j_{4}$,

(iii) $x_{i_{1}} x_{i_{2}} \ldots x_{i_{k}} \omega\left(x_{j_{1}}, x_{j_{1}}, x_{j_{2}}, x_{j_{3}}\right), i_{1} \leq i_{2} \leq \ldots \leq i_{k}, j_{2}<j_{3}$,

(iv) $x_{i_{1}} x_{i_{2}} \ldots x_{i_{k}}\left[x_{j_{1}}, x_{j_{2}}\right] \ldots\left[x_{j_{2 l-1}}, x_{l_{2 l}}\right]\left[x_{j_{2 l+1}}, x_{j_{2 l+2}}, x_{j_{2 l+3}}\right]$,

$k \geq 0, l \geq 1, i_{1} \leq i_{2} \leq \ldots \leq i_{k}, j_{1}<j_{2}<\ldots<j_{2 l+3}$. 
Seja $h$ um polinômio qualquer de (i)-(iv), que é homogêneo de grau 1 em $x_{1}$. Se existirem polinômios $h_{1}=h_{1}\left(x_{2}, \ldots, x_{n}\right) \in F\langle X\rangle, h_{2} \in\left\langle\left[x_{1}, x_{2}, x_{3}\right]\right\rangle^{T S}, h_{3} \in$ $\left\langle\omega\left(x_{1}, x_{2}, x_{3}, x_{4}\right)\right\rangle^{T S}$ e $h_{4} \in T^{(4)}$ tais que $h=h_{1} x_{1}+h_{2}+h_{3}+h_{4}$, então é fácil ver que existirão polinômios $g_{1}=g_{1}\left(x_{2}, \ldots, x_{n}\right) \in F\langle X\rangle$ (que não depende de $x_{1}$ ), $g_{2} \in$ $\left\langle\left[x_{1}, x_{2}, x_{3}\right]\right\rangle^{T S}, g_{3} \in\left\langle\omega\left(x_{1}, x_{2}, x_{3}, x_{4}\right)\right\rangle^{T S}$ e $g_{4} \in T^{(4)}$ tais que $f=x_{1} g_{1}+g_{2}+g_{3}+g_{4}$ e o lema estará provado.

Observe que se $i_{1}=1$ em cada polinômio $h$ da forma (i)-(iv), então podemos escrever $h=x_{1} h_{1}$, onde $h_{1}=h_{1}\left(x_{2}, \ldots, x_{n}\right)$ é a "parte de $h$ que está à direita de $x_{1}$ ". Como $h_{1}$ não depende de $x_{1}$ o polinômio $h$ está na forma desejada. Assim, é suficiente considerar os polinômios $h$ nos quais $i_{1} \neq 1$, isto é, a variável $x_{1}$ ocorre na "parte comutador" do polinômio $h$.

Seja $h$ um polinômio da forma (i). Então $j_{2}=1$. Fixemos $a=-x_{i_{1}} \ldots x_{i_{k}}$. Assim $h=-a\left[x_{j_{1}}, x_{1}, x_{j_{3}}\right]=a\left[x_{1}, x_{j_{1}} x_{j_{3}}\right]$. Pela Proposição 1.17 (i), temos

$$
\begin{aligned}
{\left[a x_{1}, x_{j_{1}}, x_{j_{3}}\right] } & =a\left[x_{1}, x_{j_{1}}, x_{j_{3}}\right]+\left[a, x_{j_{1}}\right]\left[x_{1}, x_{j_{3}}\right]+\left[a, x_{j_{3}}\right]\left[x_{1}, x_{j_{1}}\right]+\left[a, x_{j_{1}}, x_{j_{3}}\right] x_{1} \\
& =a\left[x_{1}, x_{j_{1}}, x_{j_{3}}\right]-\omega\left(a, x_{j_{3}}, x_{j_{1}}, x_{1}\right)+x_{1}\left[a, x_{j_{1}}, x_{j_{3}}\right]+\left[a, x_{j_{1}}, x_{j_{3}}, x_{1}\right]
\end{aligned}
$$

Pondo $h_{1}=-\left[a, x_{j_{1}}, x_{j_{3}}\right], h_{2}=\left[a x_{1}, x_{j_{1}}, x_{j_{3}}\right], h_{3}=\omega\left(a, x_{j_{3}}, x_{j_{1}}, x_{1}\right)$ e $h_{4}=\left[a, x_{j_{1}}, x_{j_{3}}, x_{1}\right]$, segue que

$$
h=x_{1} h_{1}+h_{2}+h_{3}+h_{4},
$$

onde $h_{1} \in F\langle X\rangle$ não depende de $x_{1}, h_{2} \in\left\langle\left[x_{1}, x_{2}, x_{3}\right]\right\rangle^{T S}, h_{3} \in\left\langle\omega\left(x_{1}, x_{2}, x_{3}, x_{4}\right)\right\rangle^{T S} \mathrm{e}$ $h_{4} \in T^{(4)}$.

Seja $h$ um polinômio da forma (ii). Então $j_{1}=1$. Fixemos $a=x_{i_{1}} \ldots x_{i_{k}}$. Assim $h=a \omega\left(x_{1}, x_{j_{2}}, x_{j_{3}}, x_{j_{4}}\right)$ ou $h=a \omega\left(x_{1}, x_{j_{2}}, x_{j_{4}}, x_{j_{3}}\right)$. É suficiente considerar $h=$ $a \omega\left(x_{1}, x_{j_{2}}, x_{j_{3}}, x_{j_{4}}\right)$. Pelo Lema 1.19 (i), temos

$$
\begin{aligned}
\omega\left(a x_{1}, x_{j_{2}}, x_{j_{3}}, x_{j_{4}}\right) & =a \omega\left(x_{1}, x_{j_{2}}, x_{j_{3}}, x_{j_{4}}\right)+x_{1} \omega\left(a, x_{j_{2}}, x_{j_{3}}, x_{j_{4}}\right)+ \\
& +\left[a, x_{j_{2}}, x_{1}\right]\left[x_{j_{3}}, x_{j_{4}}\right]+\left[a, x_{j_{3}}, x_{1}\right]\left[x_{j_{2}}, x_{j_{4}}\right] .
\end{aligned}
$$

Pondo $h_{1}=-\omega\left(a, x_{j_{2}}, x_{j_{3}}, x_{j_{4}}\right), h_{2}=\omega\left(a x_{1}, x_{j_{2}}, x_{j_{3}}, x_{j_{4}}\right)$ e $h_{3}=-\left[a, x_{j_{2}}, x_{1}\right]\left[x_{j_{3}}, x_{j_{4}}\right]+$ $\left[a, x_{j_{3}}, x_{1}\right]\left[x_{j_{2}}, x_{j_{4}}\right]$, temos

$$
h=x_{1} h_{1}+h_{2}+h_{3},
$$

onde $h_{1} \in F\langle X\rangle$ não depende de $x_{1}, h_{2} \in\left\langle\omega\left(x_{1}, x_{2}, x_{3}, x_{4}\right)\right\rangle^{T S}$ e $h_{3} \in T^{(4)}$ (Lema 1.32).

Seja $h$ um polinômio da forma (iii). Então $j_{2}=1$. Como $\omega\left(x_{j_{1}}, x_{j_{1}}, x_{1}, x_{j_{3}}\right) \equiv$ $\omega\left(x_{1}, x_{j_{3}}, x_{j_{1}}, x_{j_{1}}\right)\left(\bmod T^{(4)}\right)$ (veja a relação $\left.(2-52)\right)$, podemos usar o mesmo argumento do caso anterior.

Seja $h$ um polinômio da forma (iv). Fixemos $a=x_{i_{2}} \ldots x_{i_{k}}$. Fixemos também 
$b=\left[x_{j_{3}}, x_{j_{4}}\right] \ldots\left[x_{j_{2 l-1}}, x_{j_{2 l}}\right]$ se $l>1$ e $b=1$ se $l=1$. Em qualquer dos casos temos

$$
h=a\left[x_{1}, x_{j_{2}}\right] b\left[x_{j_{2 l+1}}, x_{j_{2 l+2}}, x_{j_{2 l+3}}\right]
$$

Observe que

$$
\begin{aligned}
{\left[a x_{1}, x_{j_{2}}\right] b\left[x_{j_{2 l+1}}, x_{j_{2 l+2}}, x_{j_{2 l+3}}\right] } & =a\left[x_{1}, x_{j_{2}}\right] b\left[x_{j_{2 l+1}}, x_{j_{2 l+2}}, x_{j_{2 l+3}}\right]+ \\
& +\left[a, x_{j_{2}}\right] x_{1} b\left[x_{j_{2 l+1}}, x_{j_{2 l+2}}, x_{j_{2 l+3}}\right] \\
& =a\left[x_{1}, x_{j_{2}}\right] b\left[x_{j_{2 l+1}}, x_{j_{2 l+2}}, x_{j_{2 l+3}}\right]+ \\
& +x_{1}\left[a, x_{j_{2}}\right] b\left[x_{j_{2 l+1}}, x_{j_{2 l+2}}, x_{j_{2 l+3}}\right]+ \\
& +\left[a, x_{j_{2}}, x_{1}\right] b\left[x_{j_{2 l+1}}, x_{j_{2 l+2}}, x_{j_{2 l+3}}\right]
\end{aligned}
$$

e seja $h_{1}=-\left[a, x_{j_{2}}\right] b\left[x_{j_{2 l+1}}, x_{j_{2 l+2}}, x_{j_{2 l+3}}\right]$. Como $\left[a, x_{j_{2}}, x_{1}\right] b\left[x_{j_{2 l+1}}, x_{j_{2 l+2}}, x_{j_{2 l+3}}\right]=$ $b\left[a, x_{j_{2}}, x_{1}\right]\left[x_{j_{2 l+1}}, x_{j_{2 l+2}}, x_{j_{2 l+3}}\right]+\left[a, x_{j_{2}}, x_{1}, b\right]\left[x_{j_{2 l+1}}, x_{j_{2 l+2}}, x_{j_{2 l+3}}\right] \in T^{(4)} \quad$ (Lema 1.32), segue de (2-78) que

$$
h \equiv x_{1} h_{1}+\left[a x_{1}, x_{j_{2}}\right] b\left[x_{j_{2 l+1}}, x_{j_{2 l+2}}, x_{j_{2 l+3}}\right] \quad\left(\bmod T^{(4)}\right) .
$$

Agora

$$
\begin{aligned}
{\left[\left[a x_{1}, x_{j_{2}}\right] b x_{j_{2 l+1}}, x_{j_{2 l+2}}, x_{j_{2 l+3}}\right] } & =\left[a x_{1}, x_{j_{2}}\right] b\left[x_{j_{2 l+1}}, x_{j_{2 l+2}}, x_{j_{2 l+3}}\right]+ \\
& +\left[\left[a x_{1}, x_{j_{2}}\right] b, x_{j_{2 l+2}}\right]\left[x_{j_{2 l+1}}, x_{j_{2 l+3}}\right]+ \\
& +\left[\left[a x_{1}, x_{j_{2}}\right] b, x_{j_{2 l+3}}\right]\left[x_{j_{2 l+1}}, x_{2 l+2}\right]+ \\
& +\left[\left[a x_{1}, x_{j_{2}}\right] b, x_{j_{2 l+2}}, x_{j_{2 l+3}}\right] x_{j_{2 l+1}} \\
& =\left[a x_{1}, x_{j_{2}}\right] b\left[x_{j_{2 l+1}}, x_{j_{2 l+2}}, x_{j_{2 l+3}}\right]- \\
& -\omega\left(\left[a x_{1}, x_{j_{2}}\right] b, x_{j_{2 l+2}}, x_{j_{2 l+3}}, x_{j_{2 l+2}}\right)+ \\
& +\left[\left[a x_{1}, x_{j_{2}}\right] b, x_{j_{2 l+2}}, x_{j_{2 l+3}}\right] x_{j_{2 l+1}} .
\end{aligned}
$$

Sejam $h_{2}=\left[\left[a x_{1}, x_{j_{2}}\right] b x_{j_{2 l+1}}, x_{j_{2 l+2}}, x_{j_{2 l+3}}\right], \quad h_{3}=-\omega\left(\left[a x_{1}, x_{j_{2}}\right] b, x_{j_{2 l+2}}, x_{j_{2 l+3}}, x_{j_{2 l+2}}\right)$. Usando a relação $\left[\left[x_{1}, x_{2}\right],\left[x_{3}, x_{4}\right]\right]=\left[x_{1}, x_{2}, x_{3}, x_{4}\right]-\left[x_{1}, x_{2}, x_{4}, x_{3}\right] \in T^{(4)}$ e a igualdade $[a b, c]=a[b, c]+[a, c] b$, não é difícil ver que $\left[\left[a x_{1}, x_{j_{2}}\right] b, x_{j_{2 l+2}}, x_{j_{2 l+3}}\right] x_{j_{2 l+1}} \in T^{(4)}$. Assim, segue de (2-80) que

$$
\left[a x_{1}, x_{j_{2}}\right] b\left[x_{j_{2 l+1}}, x_{j_{2 l+2}}, x_{j_{2 l+3}}\right] \equiv h_{2}+h_{3} \quad\left(\bmod T^{(4)}\right)
$$

Finalmente, vemos de $(2-79)$ e $(2-81)$ que existem $h_{2} \in\left\langle\left[x_{1}, x_{2}, x_{3}\right]\right\rangle^{T S}, h_{3} \in$ 
$\left\langle\omega\left(x_{1}, x_{2}, x_{3}, x_{4}\right)\right\rangle^{T S}$ e $h_{4} \in T^{(4)}$ tais que

$$
h=x_{1} h_{1}+h_{2}+h_{3}+h_{4},
$$

e isso conclui a demonstração do lema.

Lema 2.19. Sejam $F$ um corpo de característica 3 e $g=g\left(x_{2}, \ldots, x_{n}\right) \in F\langle X\rangle$ um polinômio que não depende de $x_{1}$. Se $x_{1} g+T^{(4)}$ for central em $Q_{4}=F\langle X\rangle / T^{(4)}$, então $g \in\left\langle x_{1}\left[x_{2}, x_{3}, x_{3}\right]\right\rangle^{T S}+\left\langle x_{1} \omega\left(x_{2}, x_{3}, x_{4}, x_{5}\right)\right\rangle^{T S}+T^{(4)}$.

Demonstração. Como $x_{1} g+T^{(4)}$ é central em $Q_{4}$, temos $\left[x_{0}, x_{1} g\right] \equiv 0\left(\bmod T^{(4)}\right)$. Assim, usando a igualdade $[a, b c]=b[a, c]+[a, b] c$, vemos que $x_{1}\left[x_{0}, g\right]+\left[x_{0}, x_{1}\right] g \equiv 0$ $\left(\bmod T^{(4)}\right)$, isto é

$$
x_{1}\left[x_{0}, g\right] \equiv-\left[x_{0}, x_{1}\right] g \quad\left(\bmod T^{(4)}\right) .
$$

Agora, como $T^{(4)} \subseteq T^{(3)}$, segue que $x_{1} g+T^{(3)}$ também é central em $Q_{3}=F\langle X\rangle / T^{(3)}$. Assim, o Lema 1.26 garante que $g \in T^{(3)}$. Pelo Lema 2.17, $g$ é uma combinação linear, módulo $T^{(4)}$, de polinômios da forma:

(i) $x_{i_{1}} x_{i_{2}} \ldots x_{i_{k}}\left[x_{j_{1}}, x_{j_{2}}, x_{j_{2}}\right], i_{1} \leq i_{2} \leq \ldots \leq i_{k}, j_{1} \neq j_{2}$,

(ii) $x_{i_{1}} x_{i_{2}} \ldots x_{i_{k}}\left[x_{j_{1}}, x_{j_{2}}, x_{j_{3}}\right], i_{1} \leq i_{2} \leq \ldots \leq i_{k}, j_{1}>j_{2} \leq j_{3}$,

(iii) $x_{i_{1}} x_{i_{2}} \ldots x_{i_{k}} \omega\left(x_{j_{1}}, x_{j_{2}}, x_{j_{3}}, x_{j_{4}}\right) ; x_{i_{1}} x_{i_{2}} \ldots x_{i_{k}} \omega\left(x_{j_{1}}, x_{j_{2}}, x_{j_{4}}, x_{j_{3}}\right)$,

$i_{1} \leq i_{2} \leq \ldots \leq i_{k}, j_{1}<j_{2}<j_{3}<j_{4}$

$x_{i_{1}} x_{i_{2}} \ldots x_{i_{k}} \omega\left(x_{j_{1}}, x_{j_{1}}, x_{j_{2}}, x_{j_{3}}\right), i_{1} \leq i_{2} \leq \ldots \leq i_{k}, j_{2}<j_{3}$,

(iv) $x_{i_{1}} x_{i_{2}} \ldots x_{i_{k}}\left[x_{j_{1}}, x_{j_{2}}\right] \ldots\left[x_{j_{2 l-1}}, x_{l_{2 l}}\right]\left[x_{j_{2 l+1}}, x_{j_{2 l+2}}, x_{j_{2 l+3}}\right]$,

$i_{1} \leq i_{2} \leq \ldots \leq i_{k}, j_{1}<j_{2}<\ldots<j_{2 l+3} k \geq 0, l \geq 1$.

Vamos analisar primeiramente o lado esquerdo de (2-82); e para fazer isso precisamos provar algumas afirmações.

Afirmação 1: Se $h_{1}$ é um polinômio da forma (i), então $h_{1}+T^{(4)}$ é central em $Q_{4}$. Isso segue do fato de $a_{1}\left[a_{2}, a_{3}, a_{3}\right]+T^{(4)}$ ser central em $Q_{4}$ (conforme o Lema $2.16)$.

Afirmação 2: Se $h_{2}$ é um polinômio da forma (ii), então $\left[x_{0}, h_{2}\right]$ é uma combinação linear, módulo $T^{(4)}$, de polinômios da forma (iv). De fato, seja $h_{2}=x_{i_{1}} x_{i_{2}} \ldots x_{i_{k}}\left[x_{j_{1}}, x_{j_{2}}, x_{j_{3}}\right]$. Usando a igualdade $\left[a_{1} a_{2} \ldots a_{n}, a\right]=$ $\sum_{i=1}^{n} a_{1} \ldots a_{i-1}\left[a_{i}, a\right] a_{i+1} \ldots a_{n}$ (Lema 1.17 (ii)), temos

$$
\begin{aligned}
{\left[x_{0}, h_{2}\right] } & =\left[x_{0}, x_{i_{1}}\right] x_{i_{2}} \ldots x_{i_{k}}\left[x_{j_{1}}, x_{j_{2}}, x_{j_{3}}\right]+\ldots+x_{i_{1}} \ldots x_{i_{k-1}}\left[x_{0}, x_{i_{k}}\right]\left[x_{j_{1}}, x_{j_{2}}, x_{j_{3}}\right]+ \\
& +x_{i_{1}} \ldots x_{i_{k}}\left[x_{0},\left[x_{j_{1}}, x_{j_{2}}, x_{j_{3}}\right]\right] .
\end{aligned}
$$


Observe que $\left[a_{1}, a_{2}, a_{3}\right]+T^{(4)}$ e $\left[a_{1}, a_{2}\right]\left[a_{3}, a_{4}, a_{5}\right]+T^{(4)}$ são centrais em $Q_{4}$. Como $x_{i_{1}} \ldots x_{i_{k}}\left[x_{0},\left[x_{j_{1}}, x_{j_{2}}, x_{j_{3}}\right]\right]$ pertence a $T^{(4)}$, podemos escrever

$$
\left[x_{0}, h_{2}\right] \equiv x_{i_{1}} \ldots x_{i_{k}}\left[x_{0}, x_{i_{1}}\right]\left[x_{j_{1}}, x_{j_{2}}, x_{j_{3}}\right]+\ldots+x_{i_{1}} \ldots x_{i_{k-1}}\left[x_{0}, x_{i_{k}}\right]\left[x_{j_{1}}, x_{j_{2}}, x_{j_{3}}\right] \quad\left(\bmod T^{(4)}\right)
$$

Agora, usando o Corolário 1.33 (ii), podemos ordenar os índices de cada variável dentro dos comutadores da expressão (2-83). Assim, $\left[x_{0}, h_{2}\right]$ é uma combinação linear, módulo $T^{(4)}$, de polinômios da forma (iv).

Afirmação 3: Se $h_{3}$ é um polinômio de (iii), então $h_{3}+T^{(4)}$ é central em $Q_{4}$. Isso segue do fato de $a_{1} \omega\left(a_{2}, a_{3}, a_{4}, a_{5}\right)+T^{4}$ ser central em $Q_{4}$ (conforme Lema 2.16).

Afirmação 4: Se $h_{4}$ é um polinômio da forma (iv), então $\left[x_{0}, h_{4}\right]$ é uma combinação linear, módulo $T^{(4)}$, de polinômios da forma (iv). De fato, seja $h_{4}=x_{i_{1}} x_{i_{2}} \ldots x_{i_{k}}\left[x_{j_{1}}, x_{j_{2}}\right] \ldots\left[x_{j_{2 l-1}}, x_{l_{2 l}}\right]\left[x_{j_{2 l+1}}, x_{j_{2 l+2}}, x_{j_{2 l+3}}\right]$. Usando a igualdade $\left[a_{1} a_{2} \ldots a_{n}, a\right]=\sum_{i=1}^{n} a_{1} \ldots a_{i-1}\left[a_{i}, a\right] a_{i+1} \ldots a_{n}$ (Lema 1.17 (ii)), temos

$$
\begin{aligned}
{\left[x_{0}, h_{4}\right] } & =\left[x_{0}, x_{i_{1}}\right] x_{i_{2}} \ldots x_{i_{k}}\left[x_{j_{1}}, x_{j_{2}}\right] \ldots\left[x_{j_{2 l-1}}, x_{l_{2 l}}\right]\left[x_{j_{2 l+1}}, x_{j_{2 l+2}}, x_{j_{2 l+3}}\right]+\ldots+ \\
& +x_{i_{1}} \ldots x_{i_{k-1}}\left[x_{0}, x_{i_{k}}\right]\left[x_{j_{1}}, x_{j_{2}}\right] \ldots\left[x_{j_{2 l-1}}, x_{l_{2 l}}\right]\left[x_{j_{2 l+1}}, x_{j_{2 l+2}}, x_{j_{2 l+3}}\right]+ \\
& +x_{i_{1}} x_{i_{2}} \ldots x_{i_{k}}\left[x_{0},\left[x_{j_{1}}, x_{j_{2}}\right] \ldots\left[x_{j_{2 l-1}}, x_{l_{2 l}}\right]\left[x_{j_{2 l+1}}, x_{j_{2 l+2}}, x_{j_{2 l+3}}\right]+\ldots+\right. \\
& +x_{i_{1}} x_{i_{2}} \ldots x_{i_{k}}\left[x_{j_{1}}, x_{j_{2}}\right] \ldots\left[x_{j_{2 l-1}}, x_{l_{2 l}}\right]\left[x_{0},\left[x_{j_{2 l+1}}, x_{j_{2 l+2}}, x_{j_{2 l+3}}\right]\right]
\end{aligned}
$$

Como $\left[a_{1}, a_{2}, a_{3}\right]+T^{(4)}$ é central em $Q_{4}$ e $\left[a_{1}, a_{2}, a_{3}\right]\left[a_{4}, a_{5}, a_{6}\right] \in T^{(4)}$ (Lema 1.32), obtemos

$$
\begin{aligned}
{\left[x_{0}, h_{4}\right] } & \equiv x_{i_{2}} \ldots x_{i_{k}}\left[x_{0}, x_{i_{1}}\right]\left[x_{j_{1}}, x_{j_{2}}\right] \ldots\left[x_{j_{2 l-1}}, x_{l_{2 l}}\right]\left[x_{j_{2 l+1}}, x_{j_{2 l+2}}, x_{j_{2 l+3}}\right]+\ldots+ \\
& +x_{i_{1}} \ldots x_{i_{k-1}}\left[x_{0}, x_{k}\right]\left[x_{j_{1}}, x_{j_{2}}\right] \ldots\left[x_{j_{2 l-1}}, x_{l_{2 l}}\right]\left[x_{j_{2 l+1}}, x_{j_{2 l+2}}, x_{j_{2 l+3}}\right] \quad\left(\bmod T^{(4)}\right)
\end{aligned}
$$

Usando o Corolário 1.33 (ii) para ordenar os índices de cada variável dentro dos comutadores da expressão (2-84), vemos que $\left[x_{0}, h_{4}\right]$ é uma combinação linear, módulo $T^{(4)}$, de polinômios da forma (iv).

Lembrando que $g$ é uma combinação linear, módulo $T^{(4)}$, de polinômios em (i)-(iv), segue das Afirmações 1 à 4, o seguinte

Afirmação 5: O polinômio $x_{1}\left[x_{0}, g\right]$ de (2-82) é uma combinação linear, módulo $T^{(4)}$, de polinômios da forma (iv) nos quais $x_{1}$ não ocorre em nenhum comutador.

Agora, vejamos o lado direito de (2-82). Como antes, sejam $h_{1}, h_{2}, h_{3}$ e $h_{4}$ polinômios das formas (i),(ii),(iii) e (iv), respectivamente. Vamos provar mais algumas afirmações.

Afirmação 6: O polinômio $\left[x_{0}, x_{1}\right] h_{1}$ pertence a $T^{(4)}$. 
De fato, como $\left[a_{1}, a_{2}, a_{3}\right]+T^{(4)}$ é central em $Q_{4}$ e $\left[a_{1}, a_{2}\right]\left[a_{3}, a_{4}, a_{4}\right]$ pertence a $T^{(4)}$ (Corolário 1.33 (ii)), temos

$$
\begin{aligned}
{\left[x_{0}, x_{1}\right] h_{1} } & =\left[x_{0}, x_{1}\right] x_{i_{1}} x_{i_{2}} \ldots x_{i_{k}}\left[x_{j_{1}}, x_{j_{2}}, x_{j_{2}}\right] \equiv x_{i_{1}} x_{i_{2}} \ldots x_{i_{k}}\left[x_{0}, x_{1}\right]\left[x_{j_{1}}, x_{j_{2}}, x_{j_{2}}\right] \quad\left(\bmod T^{(4)}\right) \\
& \equiv 0 \quad\left(\bmod T^{(4)}\right) .
\end{aligned}
$$

Afirmação 7: O polinômio $\left[x_{0}, x_{1}\right] h_{2}$ é, módulo $T^{(4)}$, um múltiplo escalar de algum polinômio da forma (iv).

De fato, como $\left[a_{1}, a_{2}, a_{3}\right]+T^{(4)}$ é central em $Q_{4}$, temos

$$
\left[x_{0}, x_{1}\right] h_{2}=\left[x_{0}, x_{1}\right] x_{i_{1}} x_{i_{2}} \ldots x_{i_{k}}\left[x_{j_{1}}, x_{j_{2}}, x_{j_{3}}\right] \equiv x_{i_{1}} x_{i_{2}} \ldots x_{i_{k}}\left[x_{0}, x_{1}\right]\left[x_{j_{1}}, x_{j_{2}}, x_{j_{3}}\right] \quad\left(\bmod T^{(4)}\right)
$$

Usando o Corolário 1.33 (ii) para ordenar os índices no comutador triplo, vemos que $\left[x_{0}, x_{1}\right] h_{2}$ é, módulo $T^{(4)}$, um múltiplo escalar de algum polinômio da forma (iv).

Afirmação 8: O polinômio $\left[x_{0}, x_{1}\right] h_{3}$ pertence a $T^{(4)}$.

De fato, é suficiente considerar $h_{3}=x_{i_{1}} x_{i_{2}} \ldots x_{i_{k}} \omega\left(x_{j_{1}}, x_{j_{2}}, x_{j_{3}}, x_{j_{4}}\right)$. Como $\omega\left(x_{j_{1}}, x_{j_{2}}, x_{j_{3}}, x_{j_{4}}\right)+T^{(4)}$ é central em $Q_{4}$ (Lema 2.16) e $\left[x_{0}, x_{1}\right] \omega\left(x_{j_{1}}, x_{j_{2}}, x_{j_{3}}, x_{j_{4}}\right)$ pertence a $T^{(4)}$ (Lema 1.32), temos

$$
\begin{aligned}
{\left[x_{0}, x_{1}\right] h_{3} } & =\left[x_{0}, x_{1}\right] x_{i_{1}} x_{i_{2}} \ldots x_{i_{k}} \omega\left(x_{j_{1}}, x_{j_{2}}, x_{j_{3}}, x_{j_{4}}\right) \\
& \equiv\left[x_{0}, x_{1}\right] \omega\left(x_{j_{1}}, x_{j_{2}}, x_{j_{3}}, x_{j_{4}}\right) x_{i_{1}} x_{i_{2}} \ldots x_{i_{k}} \quad\left(\bmod T^{(4)}\right)=0 .
\end{aligned}
$$

Afirmação 9: O polinômio $\left[x_{0}, x_{1}\right] h_{4}$ é, módulo $T^{(4)}$, um múltiplo escalar de algum polinômio da forma (iv).

De fato, como $\left[a_{1}, a_{2}, a_{3}\right]+T^{(4)}$ é central em $Q_{4}$ e $\left[a_{1}, a_{2}, a_{3}\right]\left[a_{4}, a_{5}, a_{6}\right]$ pertence a $T^{(4)}$ (Lema 1.32), é fácil verificar que

$$
\begin{aligned}
{\left[x_{0}, x_{1}\right] h_{4} } & =\left[x_{0}, x_{1}\right] x_{i_{1}} x_{i_{2}} \ldots x_{i_{k}}\left[x_{j_{1}}, x_{j_{2}}\right] \ldots\left[x_{j_{2 l-1}}, x_{l_{2 l}}\right]\left[x_{j_{2 l+1}}, x_{j_{2 l+2}}, x_{j_{2 l+3}}\right] \\
& \equiv x_{i_{1}} x_{i_{2}} \ldots x_{i_{k}}\left[x_{0}, x_{1}\right]\left[x_{j_{1}}, x_{j_{2}}\right] \ldots\left[x_{j_{2 l-1}}, x_{l_{2 l}}\right]\left[x_{j_{2 l+1}}, x_{j_{2 l+2}}, x_{j_{2 l+3}}\right] \quad\left(\bmod T^{(4)}\right)
\end{aligned}
$$

onde todos os índices já estão da forma desejada. Mostramos assim que o polinômio $\left[x_{0}, x_{1}\right] h_{4}$ é, módulo $T^{(4)}$, um múltiplo escalar de algum polinômio da forma (iv).

Segue das Afirmações 6 à 9 a seguinte:

Afirmação 10: O polinômio $\left[x_{1}, x_{0}\right] g$ de (2-82) é uma combinação linear, módulo $T^{(4)}$, de polinômios da forma (iv) nos quais $x_{1}$ ocorre somente nos comutadores.

Seja $R$ o conjunto de todos os polinômios da forma (iv) nos quais a variável $x_{1}$ não ocorre na "parte comutador" e seja $S$ o conjunto de todos os polinômios da forma (iv) nos quais a variável $x_{1}$ ocorre somente na "parte comutador". Observe que $R$ e $S$ são conjuntos disjuntos. 
Pela Afirmação 5, o polinômio $x_{1}\left[x_{0}, g\right]$ é uma combinação linear, módulo $T^{(4)}$, de polinômios de $R$. Pela Afirmação 10 , o polinômio $\left[x_{0}, x_{1}\right] g$ é uma combinação linear, módulo $T^{(4)}$, de polinômios de $S$. Como a imagem dos polinômios da forma (iv) em $F\langle X\rangle / T^{(4)}$ é parte da base de $T^{(3)} / T^{(4)}$ (Lema 2.17), segue que essa imagem forma um conjunto linearmente independente. Sendo $R$ e $S$ conjuntos disjuntos, segue de $(2-82)$ que

$$
\left[x_{0}, x_{1}\right] g \equiv 0 \quad\left(\bmod T^{(4)}\right)
$$

Agora, vamos escrever

$$
g+T^{(4)}=\sum_{i=1}^{n_{1}} \alpha_{i}^{(1)} h_{i}^{(1)}+\sum_{j=1}^{n_{2}} \alpha_{j}^{(2)} h_{j}^{(2)}+\sum_{k=1}^{n_{3}} \alpha_{k}^{(3)} h_{k}^{(3)}+\sum_{l=1}^{n_{4}} \alpha_{l}^{(4)} h_{l}^{(4)}+T^{(4)}
$$

onde $h_{i}^{(1)}, h_{j}^{(2)}, h_{k}^{(3)}$ e $h_{l}^{(4)}$ são polinômios em (i), (ii), (iii) e (iv), respectivamente. Multiplicando ambos os membros da igualdade (2-86) por $\left[x_{0}, x_{1}\right]+T^{(4)}$, segue da Afirmação 10 que

$$
\left[x_{0}, x_{1}\right] g+T^{(4)}=\sum_{j=1}^{n_{2}} \alpha_{j}^{(2)} u_{j}+\sum_{l=1}^{n_{4}} \alpha_{l}^{(4)} v_{l}+T^{(4)}
$$

onde os polinômios $u_{j}$ e $v_{l}$ são da forma (iv). Mais ainda, os conjuntos de polinômios $\left\{u_{1}, u_{2}, \ldots, u_{n_{2}}\right\}$ e $\left\{v_{1}, v_{2}, \ldots, v_{n_{4}}\right\}$ são disjuntos. Assim, por (2-85) e (2-87) temos

$$
\sum_{j=1}^{n_{2}} \alpha_{j}^{(2)} u_{j}+\sum_{l=1}^{n_{4}} \alpha_{l}^{(4)} v_{l}+T^{(4)}=T^{(4)}
$$

Como $\left\{u_{1}, u_{2}, \ldots, u_{n_{2}}\right\} \cup\left\{v_{1}, v_{2}, \ldots, v_{n_{4}}\right\}$ é linearmente independente (Proposição $2.17)$, segue que todos os $\alpha_{j}^{(2)}\left(j=1, \ldots, n_{2}\right)$ e os $\alpha_{l}^{(4)}\left(l=1, \ldots, n_{4}\right)$ são nulos. Por (2-86) podemos escrever

$$
g+T^{(4)}=\sum_{i=1}^{n_{1}} \alpha_{i}^{(1)} h_{i}^{(1)}+\sum_{k=1}^{n_{3}} \alpha_{k}^{(3)} h_{k}^{(3)}+T^{(4)}
$$

Como os polinômios $h_{i}^{(1)}$ pertencem ao $T$-subespaço gerado por $x_{1}\left[x_{2}, x_{3}, x_{3}\right]$ e os polinômios $h_{k}^{(3)}$ pertencem ao $T$-subespaço gerado por $x_{1} \omega\left(x_{2}, x_{3}, x_{4}, x_{5}\right)$, segue de (2-88) que $g \in\left\langle x_{1}\left[x_{2}, x_{3}, x_{3}\right]\right\rangle^{T S}+\left\langle x_{1} \omega\left(x_{2}, x_{3}, x_{4}, x_{5}\right)\right\rangle^{T S}+T^{(4)}$.

Lema 2.20. Seja $F$ um corpo de característica 3 . Então $x_{0}^{3}+T^{(4)}$ é central na álgebra $Q_{4}=F\langle X\rangle / T^{(4)}$.

Demonstração. Usando a igualdade $[a b, c]=a[b, c]+[a, c] b$ podemos mostrar diretamente que

$$
\left[x_{0}^{3}, x_{1}\right]=3 x_{0}^{2}\left[x_{0}, x_{1}\right]+3 x_{0}\left[x_{0}, x_{1}, x_{0}\right]+\left[x_{0}, x_{1}, x_{0}, x_{0}\right]
$$


Como $\operatorname{char}(F)=3$ e $\left[x_{0}, x_{1}, x_{0}, x_{0}\right]$ pertence a $T^{(4)}$, segue que $\left[x_{0}^{3}, x_{1}\right]$ pertence a $T^{(4)}$, isto é $x_{0}^{3}+T^{(4)}$ é central em $Q_{4}$.

Lema 2.21. Sejam $F$ um corpo de característica 3 e $f=f\left(x_{1}, \ldots, x_{n}\right) \in C\left(Q_{4}\right) \cap$ $T^{(3)}$ um polinômio homogêneo de grau 1 em $x_{1}$. Então $f \in\left\langle\left[x_{1}, x_{2}, x_{3}\right]\right\rangle^{T S}+$ $\left\langle x_{1}\left[x_{2}, x_{3}, x_{3}\right]\right\rangle^{T S}+\left\langle x_{1} \omega\left(x_{2}, x_{3}, x_{4}, x_{5}\right)\right\rangle^{T S}+T^{(4)}$.

Demonstração. Como $f \in T^{(3)}$, segue do Lema 2.18 que existem polinômios $g_{1}=g_{1}\left(x_{2}, \ldots, x_{n}\right) \in F\langle X\rangle$ (que não depende de $\left.x_{1}\right), g_{2} \in\left\langle\left[x_{1}, x_{2}, x_{3}\right]\right\rangle^{T S}, g_{3} \in$ $\left\langle\omega\left(x_{1}, x_{2}, x_{3}, x_{4}\right)\right\rangle^{T S}$ e $g_{4} \in T^{(4)}$ tais que

$$
f=x_{1} g_{1}+g_{2}+g_{3}+g_{4}
$$

É claro que $g_{2}+T^{(4)}$ e $g_{4}+T^{(4)}$ são centrais em $Q_{4}$. Como $f+T^{(4)}$ e $g_{3}+T^{(4)}$ também são centrais em $Q_{4}$ (hipótese e Lema 2.16, respectivamente), segue de (2-89) que $x_{1} g_{1}+T^{(4)}$ é central em $Q_{4}$. Assim, pelo Lema 2.19, segue que $g_{1} \in\left\langle x_{1}\left[x_{2}, x_{3}, x_{3}\right]\right\rangle^{T S}+\left\langle x_{1} \omega\left(x_{2}, x_{3}, x_{4}, x_{5}\right)\right\rangle^{T S}+T^{(4)}$. Como $\left\langle\omega\left(x_{2}, x_{3}, x_{4}, x_{5}\right)\right\rangle^{T S} \subseteq$ $\left\langle x_{1} \omega\left(x_{2}, x_{3}, x_{4}, x_{5}\right)\right\rangle^{T S}$, segue de (2-89) que $f \in\left\langle\left[x_{1}, x_{2}, x_{3}\right]\right\rangle^{T S}+\left\langle x_{1}\left[x_{2}, x_{3}, x_{3}\right]\right\rangle^{T S}+$ $\left\langle x_{1} \omega\left(x_{2}, x_{3}, x_{4}, x_{5}\right)\right\rangle^{T S}+T^{(4)}$.

Lema 2.22. Sejam $F$ um corpo de característica 3 e $f=f\left(x_{1}, \ldots, x_{n}\right) \in C\left(Q_{4}\right) \cap T^{(3)}$ um polinômio homogêneo de grau $m_{1}$ em $x_{1}$ onde $m_{1}$ não é um múltiplo de 3 . Então $f \in\left\langle\left[x_{1}, x_{2}, x_{3}\right]\right\rangle^{T S}+\left\langle x_{1}\left[x_{2}, x_{3}, x_{3}\right]\right\rangle^{T S}+\left\langle x_{1} \omega\left(x_{2}, x_{3}, x_{4}, x_{5}\right)\right\rangle^{T S}+T^{(4)}$.

Demonstração. Pela Proposição 1.16 não há perda de generalidade em supor que $f$ é multi-homogêneo de grau $m_{1}$ em $x_{1}$. Seja $I_{1}$ o $T$-subespaço gerado pelos polinômios

$$
\left[x_{1}, x_{2}, x_{3}\right], x_{1}\left[x_{2}, x_{3}, x_{3}\right], x_{1} \omega\left(x_{2}, x_{3}, x_{4}, x_{5}\right) \text { e } x_{1}\left[x_{2}, x_{3}, x_{4}, x_{5}\right]
$$

É claro que $I_{1}=\left\langle\left[x_{1}, x_{2}, x_{3}\right]\right\rangle^{T S}+\left\langle x_{1}\left[x_{2}, x_{3}, x_{3}\right]\right\rangle^{T S}+\left\langle x_{1} \omega\left(x_{2}, x_{3}, x_{4}, x_{5}\right)\right\rangle^{T S}+T^{(4)}$. Devemos mostrar que $f$ pertence a $I_{1}$. Seja $I_{2}$ o $T$-subespaço gerado pelos polinômios $x_{1}\left[x_{2}, x_{3}, x_{3}\right], x_{1} \omega\left(x_{2}, x_{3}, x_{4}, x_{5}\right)$ e $x_{1}\left[x_{2}, x_{3}, x_{4}, x_{5}\right]$. Observe que $I_{2}$ é um $T$-ideal (mas só precisaremos dele como um $T$-subespaço). Pelo Lema 2.16, temos ainda

$$
I_{2} \subseteq I_{1} \subseteq C\left(Q_{4}\right)
$$

Seja $m_{1}=3 s+r$ com $0<r<3$. Pela Proposição 2.17, a imagem dos polinômios (2-65), (2-66) e (2-68) em $F\langle X\rangle / I_{2}$ gera o espaço vetorial $T^{(3)} / I_{2}$. Usando essa informação não é difícil ver que existe um polinômio $g=g\left(x_{1}, \ldots, x_{n}\right) \in F\langle X\rangle$, multi-homogêneo de grau $r$ em $x_{1}$ tal que

$$
f+I_{2}=x_{1}^{3 s} g+I_{2}
$$


Defina o endomorfismo $\varphi$ de $F\langle X\rangle$ por $\varphi\left(x_{1}\right)=1+x_{1}$ e $\varphi\left(x_{i}\right)=x_{i}$ se $i>1$. Por (2-91), temos

$$
\varphi(f)+I_{2}=\left(1+x_{1}^{3}\right)^{s} g\left(1+x_{1}, x_{2}, \ldots, x_{n}\right)+I_{2} .
$$

Segue de $(2-90)$ e $(2-92)$ que $\left(1+x_{1}^{3}\right)^{s} g\left(1+x_{1}, x_{2}, \ldots, x_{n}\right)+T^{(4)}$ é central em $Q_{4}$. Como $g\left(x_{1}, \ldots, x_{n}\right)$ é a componente multi-homogênea de grau $r$ em $x_{1}$ do polinômio $\left(1+x_{1}^{3}\right)^{s} g\left(1+x_{1}, x_{2}, \ldots, x_{n}\right)$, segue de (2-90) e (2-92) que $g+T^{(4)}$ é central em $Q_{4}$. Seja $h=h\left(y_{1}, \ldots, y_{r}, x_{2}, \ldots, x_{n}\right)$ a linearização total de $g$ em $x_{1}$. Então $h+T^{(4)}$ é central em $Q_{4}$ e segue do Lema 2.21 que $h$ pertence a $I_{1}$. Como

$$
h\left(x_{1}, \ldots, x_{1}, x_{2}, \ldots, x_{n}\right)=r ! g\left(x_{1}, \ldots, x_{n}\right),
$$

segue que $g$ também pertence a $I_{1}$. Pelo Lema 1.17 (i), temos

$$
\begin{aligned}
{\left[x_{1}^{3 s} x_{2}, x_{3}, x_{4}\right] } & =x_{1}^{3 s}\left[x_{2}, x_{3}, x_{4}\right]+\left[x_{1}^{3 s}, x_{3}\right]\left[x_{2}, x_{4}\right]+\left[x_{1}^{3 s}, x_{4}\right]\left[x_{2}, x_{3}\right]+ \\
& +\left[x_{1}^{3 s}, x_{3}, x_{4}\right] x_{2}
\end{aligned}
$$

Segue do Lema 2.20 que $x^{3 s}+T^{(4)}$ é central em $Q_{4}$. Logo os polinômios $\left[x_{1}^{3 s}, x_{3}\right]\left[x_{2}, x_{4}\right],\left[x_{1}^{3 s}, x_{4}\right]\left[x_{2}, x_{3}\right]$ e $\left[x_{1}^{3 s}, x_{3}, x_{4}\right] x_{2}$ pertencem a $T^{(4)}$. Assim

$$
\left[x_{1}^{3 s} x_{2}, x_{3}, x_{4}\right] \equiv x_{1}^{3 s}\left[x_{2}, x_{3}, x_{4}\right] \quad\left(\bmod T^{(4)}\right)
$$

Lembrando que $g \in I_{1}$, segue de (2-93) que $x_{1}^{3 s} g$ também pertence a $I_{1}$. Finalmente, como $I_{2} \subseteq I_{1}$, segue de (2-91) que $f$ pertence a $I_{1}$, o que demonstra o lema.

Lema 2.23. Sejam $F$ um corpo de característica 3 e $I=\left\langle x_{1}\left[x_{2}, x_{3}, x_{3}\right]\right\rangle^{T S}+$ $\left\langle x_{1} \omega\left(x_{2}, x_{3}, x_{4}, x_{5}\right)\right\rangle^{T S}+T^{(4)}$. Então $\left(x_{1} \ldots x_{n}\right)^{3} \equiv x_{1}^{3} \ldots x_{n}^{3}(\bmod I)$.

Demonstração. Pode ser verificado diretamente que

$$
\left(x_{1} x_{2}\right)^{3}=x_{1}^{3} x_{2}^{3}+x_{1}^{2} x_{2}\left[x_{1}, x_{2}, x_{2}\right]+x_{1} x_{2}^{2}\left[x_{2}, x_{1}, x_{1}\right]+x_{1}\left[x_{1}, x_{2}\right]\left[x_{1}, x_{2}\right] x_{2}
$$

Como $\quad\left[\left[x_{1}, x_{2}\right]\left[x_{1}, x_{2}\right], x_{3}\right]=\left[x_{1}, x_{2}\right]\left[x_{1}, x_{2}, x_{3}\right]+\left[x_{1}, x_{2}, x_{3}\right]\left[x_{1}, x_{2}\right] \equiv$ $2\left[x_{1}, x_{2}, x_{3}\right]\left[x_{1}, x_{2}\right]\left(\bmod T^{(4)}\right)$ e $\left[x_{1}, x_{2}, x_{3}\right]\left[x_{1}, x_{2}\right] \in T^{(4)}$ (Corolário $1.33($ ii)), segue que $\left[\left[x_{1}, x_{2}\right]\left[x_{1}, x_{2}\right], x_{3}\right] \in T^{(4)}$, isto é $\left[x_{1}, x_{2}\right]\left[x_{1}, x_{2}\right]+T^{(4)}$ é central em $Q_{4}$. Voltando a (2-94), temos

$$
\left(x_{1} x_{2}\right)^{3} \equiv x_{1}^{3} x_{2}^{3}+x_{1}^{2} x_{2}\left[x_{1}, x_{2}, x_{2}\right]+x_{1} x_{2}^{2}\left[x_{2}, x_{1}, x_{1}\right]+x_{1} x_{2}\left[x_{1}, x_{2}\right]\left[x_{1}, x_{2}\right] \quad\left(\bmod T^{(4)}\right) .
$$

Observe que $x_{1} x_{2}\left[x_{1}, x_{2}\right]\left[x_{1}, x_{2}\right]=x_{1} x_{2} \omega\left(x_{1}, x_{2}, x_{1}, x_{2}\right)$. Assim os polinômios $x_{1}^{2} x_{2}\left[x_{1}, x_{2}, x_{2}\right], x_{1} x_{2}^{2}\left[x_{2}, x_{1}, x_{1}\right]$ e $x_{1} x_{2}\left[x_{1}, x_{2}\right]\left[x_{1}, x_{2}\right]$ pertencem a $I$. Logo (2-95) implica 
que

$$
\left(x_{1} x_{2}\right)^{3} \equiv x_{1}^{3} x_{2}^{3} \quad(\bmod I)
$$

Como $\omega\left(x_{1}, x_{2}, x_{3}, x_{4}\right)+T^{(4)}$ é central em $Q_{4}=F\langle X\rangle / T^{(4)}$ (Lema 2.16), usando a igualdade $a b=b a+[a, b]$ é fácil verificar que $I$ é um ideal bilateral de $F\langle X\rangle$. Agora, usando (2-96) como base de indução, segue facilmente que $\left(x_{1} \ldots x_{n}\right)^{3} \equiv$ $x_{1}^{3} \ldots x_{n}^{3}(\bmod I)$.

Lembrando que

$$
q_{n}=q_{n}\left(x_{1}, \ldots, x_{2 n}\right)=x_{1}^{2}\left[x_{1}, x_{2}\right] x_{2}^{2} \ldots x_{2 n-1}^{2}\left[x_{2 n-1}, x_{2 n}\right] x_{2 n}^{2}
$$

vamos definir também $u_{0}=u_{0}\left(x_{1}, x_{2}, x_{3}\right)=x_{1}^{2} x_{2}^{2} x_{3}^{2}\left[x_{1}, x_{2}, x_{3}\right]$, e para cada $n \geq 1$,

$$
u_{n}=u_{n}\left(x_{1}, \ldots, x_{2 n+3}\right)=q_{n}\left(x_{1}, x_{2}, \ldots, x_{2 n}\right) u_{0}\left(x_{2 n+1}, x_{2 n+2}, x_{2 n+3}\right) .
$$

Lema 2.24. Sejam $F$ um corpo de característica 3 e $f=f\left(x_{1}, \ldots, x_{n}\right) \in T^{(3)}$ um polinômio multi-homogêneo de multi-grau $\left(m_{1}, \ldots, m_{n}\right)$ onde $m_{i}$ é divisivel por 3 para cada $i=1, \ldots, n$. Então $f$ pertence ao $T$-subespaço gerado pelos polinômios

$$
\begin{aligned}
& x_{1}\left[x_{2}, x_{3}, x_{3}\right], x_{1} \omega\left(x_{2}, x_{3}, x_{4}, x_{5}\right), x_{1}\left[x_{2}, x_{3}, x_{4}, x_{5}\right], \\
& x_{0}^{3} u_{0}, x_{0}^{3} u_{1}, x_{0}^{3} u_{2}, \ldots, x_{0}^{3} u_{n}, \ldots
\end{aligned}
$$

Demonstração. Como $x_{0}^{3}+T^{(4)}$ é central em $Q_{4}$ (Lema 2.20), segue da Proposição 2.17 que $f$ pode ser escrito, módulo $T^{(4)}$, como uma combinação linear dos polinômios

$$
\begin{aligned}
& x_{i_{1}}^{3 r_{1}} x_{i_{2}}^{3 r_{2}} \ldots x_{i_{k}}^{3 r_{k}} x_{j_{1}}^{2} x_{j_{2}}\left[x_{j_{1}}, x_{j_{2}}, x_{j_{2}}\right], i_{1} \leq i_{2} \leq \ldots \leq i_{k}, j_{1} \neq j_{2}, \\
& x_{i_{1}}^{3 r_{1}} x_{i_{2}}^{3 r_{2}} \ldots x_{i_{k}}^{3 r_{k}} x_{j_{1}}^{2} x_{j_{2}}^{2} x_{j_{3}}^{2}\left[x_{j_{1}}, x_{j_{2}}, x_{j_{3}}\right], i_{1} \leq i_{2} \leq \ldots \leq i_{k}, j_{1}>j_{2}<j_{3}, \\
& x_{i_{1}}^{3 r_{1}} x_{i_{2}}^{3 r_{2}} \ldots x_{i_{k}}^{3 r_{k}} x_{j_{1}}^{2} x_{j_{2}}^{2} x_{j_{3}}^{2} x_{j_{4}}^{2} \omega\left(x_{j_{1}}, x_{j_{2}}, x_{j_{3}}, x_{j_{4}}\right), \\
& x_{i_{1}}^{3 r_{1}} x_{i_{2}}^{3 r_{2}} \ldots x_{i_{k}}^{3 r_{k}} x_{j_{1}}^{2} x_{j_{2}}^{2} x_{j_{3}}^{2} x_{j_{4}}^{2} \omega\left(x_{j_{1}}, x_{j_{2}}, x_{j_{4}}, x_{j_{3}}\right), i_{1} \leq i_{2} \leq \ldots \leq i_{k}, j_{1}<j_{2}<j_{3}<j_{4}, \\
& x_{i_{1}}^{3 r_{1}} x_{i_{2}}^{3 r_{2}} \ldots x_{i_{k}}^{3 r_{k}} x_{j_{1}}^{2}\left[x_{j_{1}}, x_{j_{2}}\right] x_{j_{2}}^{2} \ldots x_{j_{2 l-1}}^{2}\left[x_{j_{2 l-1}}, x_{j_{2 l} l}\right] x_{j_{2 l}}^{2} x_{j_{2 l+1}}^{2} x_{j_{2 l+2}}^{2} x_{j_{2 l+3}}^{2}\left[x_{j_{2 l+1}}, x_{j_{2 l+2}}, x_{j_{2 l+3}}\right], \\
& i_{1} \leq i_{2} \leq \ldots \leq i_{k}, j_{1}<j_{2}<\ldots<j_{2 k+3}, k \geq 0, l \geq 1 .
\end{aligned}
$$

Os polinômios da forma (2-99) pertencem ao $T$-subespaço gerado pelo polinômio $x_{1}\left[x_{2}, x_{3}, x_{3}\right]$, que está entre aqueles de (2-97).

Seja $I$ o $T$-subespaço gerado pelos polinômios (2-97). Segue do Lema 2.23 que

$$
x_{i_{1}}^{3 r_{1}} x_{i_{2}}^{3 r_{2}} \ldots x_{i_{k}}^{3 r_{k}} x_{j_{1}}^{2} x_{j_{2}}^{2} x_{j_{3}}^{2}\left[x_{j_{1}}, x_{j_{2}}, x_{j_{3}}\right] \equiv\left(x_{i_{1}}^{r_{1}} x_{i_{2}}^{r_{2}} \ldots x_{i_{k}}^{r_{k}}\right)^{3} x_{j_{1}}^{2} x_{j_{2}}^{2} x_{j_{3}}^{2}\left[x_{j_{1}}, x_{j_{2}}, x_{j_{3}}\right] \quad(\bmod I) .
$$


Assim os polinômios da forma (2-100) pertencem ao $T$-subespaço gerado pelos polinômios $x_{0}^{3} u_{0}\left(x_{1}, x_{2}, x_{3}\right), x_{1}\left[x_{2}, x_{3}, x_{3}\right], x_{1} \omega\left(x_{2}, x_{3}, x_{4}, x_{5}\right)$ e $x_{1}\left[x_{2}, x_{3}, x_{4}, x_{5}\right]$, que estão entre aqueles de (2-97)-(2-98).

Os polinômios de (2-101) pertencem ao $T$-subespaço gerado pelo polinômio $x_{1} \omega\left(x_{2}, x_{3}, x_{4}, x_{5}\right)$, que está entre aqueles de (2-97).

Finalmente, usando o Lema 2.23, podemos verificar de modo análogo ao caso feito para os polinômios (2-100) que os polinômios da forma (2-102) pertencem ao $T$ subespaço gerado pelos polinômios $x_{0}^{3} u_{n}, n=0,1,2, \ldots$ juntamente com os polinômios $x_{1}\left[x_{2}, x_{3}, x_{3}\right], x_{1} \omega\left(x_{2}, x_{3}, x_{4}, x_{5}\right)$ e $x_{1}\left[x_{2}, x_{3}, x_{4}, x_{5}\right]$. Todos eles estão entre aqueles de $(2-$ 97)-(2-98). Isso conclui a demonstração do lema.

Lema 2.25. Seja F um corpo qualquer. Então $u_{n}+T^{(4)}$ é central na álgebra $Q_{4}=F\langle X\rangle / T^{(4)}$ para cada $n=0,1,2, \ldots$

Demonstração. Primeiro vamos mostrar que $u_{0}+T^{(4)}$ é central em $Q_{4}$. De fato, usando a igualdade $[a b, c]=a[b, c]+[a, c] b$, temos

$$
\begin{aligned}
{\left[u_{0}, x_{4}\right]=\left[x_{1}^{2} x_{2}^{2} x_{3}^{2}\left[x_{1}, x_{2}, x_{3}\right], x_{4}\right] } & =x_{1}^{2} x_{2}^{2} x_{3}^{2}\left[x_{1}, x_{2}, x_{3}, x_{4}\right]+\left[x_{1}^{2} x_{2}^{2} x_{3}^{2}, x_{4}\right]\left[x_{1}, x_{2}, x_{3}\right] \\
& \equiv\left[x_{1}^{2} x_{2}^{2} x_{3}^{2}, x_{4}\right]\left[x_{1}, x_{2}, x_{3}\right]\left(\bmod T^{(4)}\right) .
\end{aligned}
$$

Usando a igualdade $\left[a_{1} a_{2} \ldots a_{n}, a\right]=\sum_{i=1}^{n} a_{1} \ldots a_{i-1}\left[a_{i}, a\right] a_{i+1} \ldots a_{n}$ (Lema 1.17 (ii)) e observando que $\left[x_{1}, x_{2}, x_{3}\right]+T^{(4)}$ é central em $Q_{4}$, podemos escrever

$$
\begin{aligned}
{\left[u_{0}, x_{4}\right] } & \equiv\left[x_{1}, x_{4}\right] x_{1} x_{2}^{2} x_{3}^{2}\left[x_{1}, x_{2}, x_{3}\right]+x_{1}\left[x_{1}, x_{4}\right] x_{2}^{2} x_{3}^{2}\left[x_{1}, x_{2}, x_{3}\right]+\ldots+ \\
& +x_{1}^{2} x_{2}^{2}\left[x_{3}, x_{4}\right] x_{3}\left[x_{1}, x_{2}, x_{3}\right]+x_{1}^{2} x_{2}^{2} x_{3}\left[x_{3}, x_{4}\right]\left[x_{1}, x_{2}, x_{3}\right] \quad\left(\bmod T^{(4)}\right) \\
& \equiv\left[x_{1}, x_{2}, x_{3}\right]\left[x_{1}, x_{4}\right] x_{1} x_{2}^{2} x_{3}^{2}+x_{1}\left[x_{1}, x_{2}, x_{3}\right]\left[x_{1}, x_{4}\right] x_{2}^{2} x_{3}^{2}+\ldots+ \\
& +x_{1}^{2} x_{2}^{2}\left[x_{1}, x_{2}, x_{3}\right]\left[x_{3}, x_{4}\right] x_{3}+x_{1}^{2} x_{2}^{2} x_{3}\left[x_{1}, x_{2}, x_{3}\right]\left[x_{3}, x_{4}\right] \quad\left(\bmod T^{(4)}\right)
\end{aligned}
$$

Segue do Corolário 1.33 (ii) que os polinômios $\left[x_{1}, x_{2}, x_{3}\right]\left[x_{1}, x_{4}\right]$ e $\left[x_{1}, x_{2}, x_{3}\right]\left[x_{3}, x_{4}\right]$ pertencem a $T^{(4)}$. Assim $\left[u_{0}, x_{4}\right] \equiv 0\left(\bmod T^{(4)}\right)$, isto é $u_{0}+T^{(4)}$ é central em $Q_{4}$.

Agora, mostremos que $u_{1}+T^{(4)}$ é central em $Q_{4}$. De fato, usando a igualdade $[a b, c]=a[b, c]+[a, c] b$ e fato já mostrado de que $u_{0}+T^{(4)}$ é central em $Q_{4}$, obtemos

$$
\begin{aligned}
{\left[u_{1}, x_{6}\right] } & =\left[q\left(x_{1}, x_{2}\right) u_{0}\left(x_{3}, x_{4}, x_{5}\right), x_{6}\right]=q\left(x_{1}, x_{2}\right)\left[u_{0}\left(x_{3}, x_{4}, x_{5}\right), x_{6}\right]+ \\
& +\left[q\left(x_{1}, x_{2}\right), x_{6}\right] u_{0}\left(x_{3}, x_{4}, x_{5}\right) \equiv\left[q\left(x_{1}, x_{2}\right), x_{6}\right] u_{0}\left(x_{3}, x_{4}, x_{5}\right) \quad\left(\bmod T^{(4)}\right) .
\end{aligned}
$$


Usando novamente a igualdade $\left[a_{1} a_{2} \ldots a_{n}, a\right]=\sum_{i=1}^{n} a_{1} \ldots a_{i-1}\left[a_{i}, a\right] a_{i+1} \ldots a_{n}$ obtemos

$$
\begin{aligned}
{\left[u_{1}, x_{6}\right] } & \equiv\left[q\left(x_{1}, x_{2}\right), x_{6}\right] u_{0}\left(x_{3}, x_{4}, x_{5}\right) \quad\left(\bmod T^{(4)}\right) \\
& =\left[x_{1}^{2}\left[x_{1}, x_{2}\right] x_{2}^{2}, x_{6}\right] u_{0}\left(x_{3}, x_{4}, x_{5}\right) \\
& =\left(\left[x_{1}, x_{6}\right] x_{1}\left[x_{1}, x_{2}\right] x_{2}^{2}+x_{1}\left[x_{1}, x_{6}\right]\left[x_{1}, x_{2}\right] x_{2}^{2}+x_{1}^{2}\left[x_{1}, x_{2}, x_{6}\right] x_{2}^{2}+\right. \\
& \left.+x_{1}^{2}\left[x_{1}, x_{2}\right]\left[x_{2}, x_{6}\right] x_{2}+x_{1}^{2}\left[x_{1}, x_{2}\right] x_{2}\left[x_{2}, x_{6}\right]\right) x_{3}^{2} x_{4}^{2} x_{5}^{2}\left[x_{3}, x_{4}, x_{5}\right]
\end{aligned}
$$

Como $\left[x_{3}, x_{4}, x_{5}\right]+T^{(4)}$ é central em $Q_{4}$, não é difícil ver que

$$
\begin{aligned}
{\left[u_{1}, x_{6}\right] } & \equiv\left(\left[x_{3}, x_{4}, x_{5}\right]\left[x_{1}, x_{6}\right]\left[x_{1}, x_{2}\right] x_{1} x_{2}^{2}+x_{1}\left[x_{3}, x_{4}, x_{5}\right]\left[x_{1}, x_{6}\right]\left[x_{1}, x_{2}\right] x_{2}^{2}+\right. \\
& +x_{1}^{2}\left[x_{1}, x_{2}, x_{6}\right]\left[x_{3}, x_{4}, x_{5}\right] x_{2}^{2}+x_{1}^{2}\left[x_{3}, x_{4}, x_{5}\right]\left[x_{1}, x_{2}\right]\left[x_{2}, x_{6}\right] x_{2}+ \\
& \left.+x_{1}^{2}\left[x_{3}, x_{4}, x_{5}\right]\left[x_{1}, x_{2}\right]\left[x_{2}, x_{6}\right] x_{2}\right) x_{3}^{2} x_{4}^{2} x_{5}^{2}
\end{aligned}
$$

Como os polinômios $\left[x_{1}, x_{2}, x_{6}\right]\left[x_{3}, x_{4}, x_{5}\right]$ e $\left[x_{3}, x_{4}, x_{5}\right]\left[x_{1}, x_{6}\right]\left[x_{1}, x_{2}\right]$ pertencem a $T^{(4)}$ (Lema 1.32 e Corolário 1.33 (ii), respectivamente) segue que $\left[u_{1}, x_{6}\right] \equiv 0\left(\bmod T^{(4)}\right)$, isto é $u_{1}+T^{(4)}$ é central em $Q_{4}$.

Agora podemos aplicar indução sobre $n$. A base da indução é $n=1$ e já foi feita. Suponha que $u_{n}+T^{(4)}$ seja central em $Q_{4}$ para algum $n \in \mathbb{N}$ com $n>1$. Então usando a igualdade $[a b, c]=a[b, c]+[a, c] b$, temos

$$
\left[u_{n+1}, y\right]=\left[q_{n+1} u_{0}, y\right]=\left[q_{n} q u_{0}, y\right]=q_{n}\left[q u_{0}, y\right]+\left[q_{n}, y\right] q u_{0}=q_{n}\left[u_{1}, y\right]+\left[q_{n}, y\right] q u_{0} .
$$

Como $u_{0}+T^{(4)}$ é central em $Q_{4}$, usando novamente a igualdade $[a b, c]=a[b, c]+[a, c] b$ podemos escrever

$$
q_{n}\left[u_{1}, y\right]+\left[q_{n}, y\right] u_{0} q \equiv q_{n}\left[u_{1}, y\right]+\left[q_{n} u_{0}, y\right] q \quad\left(\bmod T^{(4)}\right)
$$

Como $u_{1}+T^{(4)}$ e $u_{n}+T^{(4)}=q_{n} u_{0}+T^{(4)}$ são centrais em $Q_{4}$ (base e hipótese da indução, respectivamente), segue de $(2-103)-(2-104)$ que $\left[u_{n+1}, y\right] \equiv 0\left(\bmod T^{(4)}\right)$, isto é $u_{n+1}+T^{(4)}$ é central em $Q_{4}$. Isso completa a indução e demonstra o lema.

Proposição 2.26. Seja $F$ um corpo de característica 3. Então $C\left(Q_{4}\right) \cap T^{(3)}$ é gerado como $T$-subespaço de $F\langle X\rangle$ pelos polinômios

(i) $\left[x_{1}, x_{2}, x_{3}\right], x_{1}\left[x_{2}, x_{3}, x_{3}\right], x_{1} \omega\left(x_{2}, x_{3}, x_{4}, x_{5}\right), x_{1}\left[x_{2}, x_{3}, x_{4}, x_{5}\right]$,

(ii) $x_{0}^{3} u_{0}, x_{0}^{3} u_{1}, x_{0}^{3} u_{2}, \ldots, x_{0}^{3} u_{n}, \ldots$

Demonstração. Vamos verificar primeiramente que os polinômios de (i) e (ii) pertencem a $C\left(Q_{4}\right) \cap T^{(3)}$. Segue diretamente do Lema 2.16 que os polinômios de (i) pertencem a $C\left(Q_{4}\right) \cap T^{(3)}$. 
Vejamos os polinômios de (ii). Como $x_{0}^{3}+T^{(4)}$ e $u_{n}+T^{(4)}, n=0,1,2, \ldots$ são centrais em $Q_{4}$ (Lema 1.22 (i) e Lema 2.25, respectivamente) e $C\left(Q_{4}\right) \cap T^{(3)}$ é uma álgebra (Proposição 1.13) segue que todos os polinômios de (ii) pertencem a $C\left(Q_{4}\right) \cap T^{(3)}$.

Agora vamos mostrar que os polinômios (i)-(ii) geram $C\left(Q_{4}\right) \cap T^{(3)}$ como $T$-subespaço. Observe que $C\left(Q_{4}\right) \cap T^{(3)}$ é multi-homogêneo porque $C\left(Q_{4}\right)$ e $T^{(3)}$ o são (Proposição 1.10 e Proposição 1.16, respectivamente). Seja $f=f\left(x_{1}, x_{2}, \ldots, x_{n}\right) \in$ $C\left(Q_{4}\right) \cap T^{(3)}$. Pela observação feita, podemos assumir que $f$ é um polinômio multihomogêneo de multigrau $\left(m_{1}, \ldots, m_{n}\right)$.

Suponha que algum $m_{i}$ não seja divisível por 3. Reenumerando as variáveis $x_{i}$, podemos assumir, sem perda de generalidade que $i=1$. Pelo Lema 2.22, temos

$$
f \in\left\langle\left[x_{1}, x_{2}, x_{3}\right]\right\rangle^{T S}+\left\langle x_{1}\left[x_{2}, x_{3}, x_{3}\right]\right\rangle^{T S}+\left\langle x_{1} \omega\left(x_{2}, x_{3}, x_{4}, x_{5}\right)\right\rangle^{T S}+T^{(4)} .
$$

Assim $f$ pertence ao $T$-subespaço gerado pelos polinômios (i).

Suponha agora que cada $m_{i}$ seja divisível por 3. Como vimos no Lema 2.24, $f$ pertence ao $T$-subespaço gerado pelos polinômios (2-97)-(2-98). Como esses polinômios estão entre aqueles de (i)-(ii), o lema está demonstrado.

\subsubsection{Os geradores do espaço vetorial $\left(C\left(Q_{4}\right)+T^{(3)}\right) / T^{(3)}$}

Vamos começar obtendo alguns polinômios centrais da álgebra $Q_{4}$.

Lema 2.27. Seja $F$ um corpo de característica 3. Então $q_{n}+T^{(4)}$ é central em $Q_{4}=F\langle X\rangle / T^{(4)}$ se, e somente se $n$ é divisivel por 3 .

Demonstração. Primeiramente vamos mostrar que se $n$ é divisível por 3, então $q_{n}+T^{(4)}$ é central em $Q_{4}$. Com efeito, seja $n=3 s$ com $s \in \mathbb{N}$. Como

$$
q_{n}=q_{3 s}\left(x_{1}, \ldots, x_{6 s}\right)=q_{3}\left(x_{1}, \ldots, x_{6}\right) \ldots q_{3}\left(x_{6 s-5}, \ldots, x_{6 s}\right)
$$

e $C\left(Q_{4}\right)$ é uma $T$-subálgebra (Proposição 1.13), é suficiente mostrar que $q_{3}\left(x_{1}, \ldots, x_{6}\right)+T^{(4)}$ é central em $Q_{4}$. Como $q_{3}=q_{3}\left(x_{1}, \ldots, x_{6}\right)=$ $q\left(x_{1}, x_{2}\right) q\left(x_{3}, x_{4}\right) q\left(x_{5}, x_{6}\right)$, segue da igualdade $\left[a_{1} a_{2} \ldots a_{n}, a\right]=\sum_{i=1}^{n} a_{1} \ldots a_{i-1}\left[a_{i}, a\right] a_{i+1} \ldots a_{n}$ (Lema 1.17 (ii)), que

$$
\begin{aligned}
{\left[q_{3}, x_{7}\right] } & =\left[q\left(x_{1}, x_{2}\right), x_{7}\right] q\left(x_{3}, x_{4}\right) q\left(x_{5}, x_{6}\right)+ \\
& +q\left(x_{1}, x_{2}\right)\left[q\left(x_{3}, x_{4}\right), x_{7}\right] q\left(x_{5}, x_{6}\right)+q\left(x_{1}, x_{2}\right) q\left(x_{3}, x_{4}\right)\left[q\left(x_{5}, x_{6}\right), x_{7}\right]
\end{aligned}
$$


Usando novamente o Lema 1.17 (ii) temos

$$
\begin{aligned}
{\left[q\left(x_{1}, x_{2}\right), x_{7}\right] } & =\left[x_{1}^{2}\left[x_{1}, x_{2}\right] x_{2}^{2}, x_{7}\right] \\
& =\left[x_{1}, x_{7}\right] x_{1}\left[x_{1}, x_{2}\right] x_{2}^{2}+x_{1}\left[x_{1}, x_{7}\right]\left[x_{1}, x_{2}\right] x_{2}^{2}+x_{1}^{2}\left[x_{1}, x_{2}, x_{7}\right] x_{2}^{2}+ \\
& +x_{1}^{2}\left[x_{1}, x_{2}\right]\left[x_{2}, x_{7}\right] x_{2}+x_{1}^{2}\left[x_{1}, x_{2}\right] x_{2}\left[x_{2}, x_{7}\right] \\
& =x_{1}\left[x_{1}, x_{7}\right]\left[x_{1}, x_{2}\right] x_{2}^{2}+\left[x_{1}, x_{7}, x_{1}\right]\left[x_{1}, x_{2}\right] x_{2}^{2}+x_{1}\left[x_{1}, x_{7}\right]\left[x_{1}, x_{2}\right] x_{2}^{2}+ \\
& +x_{1}^{2}\left[x_{1}, x_{2}, x_{7}\right] x_{2}^{2}-x_{1}^{2}\left[x_{2}, x_{1}\right]\left[x_{2}, x_{7}\right] x_{2}-x_{1}^{2} x_{2}\left[x_{2}, x_{1}\right]\left[x_{2}, x_{7}\right]+ \\
& +x_{1}^{2}\left[x_{1}, x_{2}, x_{2}\right]\left[x_{2}, x_{7}\right]
\end{aligned}
$$

Os polinômios $\left[x_{1}, x_{7}, x_{1}\right]\left[x_{1}, x_{2}\right] x_{2}^{2}$ e $x_{1}^{2}\left[x_{1}, x_{2}, x_{2}\right]\left[x_{2}, x_{7}\right]$ pertencem a $T^{(4)}$ (Corolário 1.33 (ii)). Como $\left[a_{1}, a_{2}\right]\left[a_{1}, a_{3}\right]=\omega\left(a_{1}, a_{2}, a_{1}, a_{3}\right)$, segue do Lema 2.16 que $\left[a_{1}, a_{2}\right]\left[a_{1}, a_{3}\right]+T^{(4)}$ é central em $Q_{4}$. Assim, por (2-107) temos

$\left[q\left(x_{1}, x_{2}\right), x_{7}\right] \equiv 2 x_{1} x_{2}^{2}\left[x_{1}, x_{7}\right]\left[x_{1}, x_{2}\right]+x_{1}^{2}\left[x_{1}, x_{2}, x_{7}\right] x_{2}^{2}-2 x_{1}^{2} x_{2}\left[x_{2}, x_{1}\right]\left[x_{2}, x_{7}\right] \quad\left(\bmod T^{(4)}\right)$.

Como $\left[a_{1}, a_{2}\right]\left[a_{1}, a_{3}\right]+T^{(4)}$ é central em $Q_{4}$ e $\left[a_{1}, a_{2}\right]\left[a_{1}, a_{3}\right]\left[a_{4}, a_{5}\right] \in T^{(4)}$ (Corolário 1.33 (i)), segue de (2-108) que

$$
\left[q\left(x_{1}, x_{2}\right), x_{7}\right] q\left(x_{3}, x_{4}\right) q\left(x_{5}, x_{6}\right) \equiv x_{1}^{2}\left[x_{1}, x_{2}, x_{7}\right] x_{2}^{2} q\left(x_{3}, x_{4}\right) q\left(x_{5}, x_{6}\right) \quad\left(\bmod T^{(4)}\right)
$$

Como $\left[a_{1}, a_{2}, a_{3}\right]+T^{(4)}$ é central em $Q_{4}$ e $\left[a_{1}, a_{2}, a_{3}\right]\left[a_{4}, a_{5}, a_{6}\right] \in T^{(4)}$ (Lema 1.32), é fácil ver à partir de (2-109) que

$$
\left[q\left(x_{1}, x_{2}\right), x_{7}\right] q\left(x_{3}, x_{4}\right) q\left(x_{5}, x_{6}\right) \equiv x_{1}^{2} x_{2}^{2} x_{3}^{2} x_{4}^{2} x_{5}^{2} x_{6}^{2}\left[x_{1}, x_{2}, x_{7}\right]\left[x_{3}, x_{4}\right]\left[x_{5}, x_{6}\right] \quad\left(\bmod T^{(4)}\right)
$$

À partir de (2-110) podemos obter também as relações

$$
\begin{gathered}
q\left(x_{1}, x_{2}\right)\left[q\left(x_{3}, x_{4}\right), x_{7}\right] q\left(x_{5}, x_{6}\right) \equiv x_{1}^{2} x_{2}^{2} x_{3}^{2} x_{4}^{2} x_{5}^{2} x_{6}^{2}\left[x_{3}, x_{4}, x_{7}\right]\left[x_{1}, x_{2}\right]\left[x_{5}, x_{6}\right] \\
\left(\bmod T^{(4)}\right) . \\
q\left(x_{1}, x_{2}\right) q\left(x_{3}, x_{4}\right)\left[q\left(x_{5}, x_{6}\right), x_{7}\right] \equiv x_{1}^{2} x_{2}^{2} x_{3}^{2} x_{4}^{2} x_{5}^{2} x_{6}^{2}\left[x_{5}, x_{6}, x_{7}\right]\left[x_{1}, x_{2}\right]\left[x_{3}, x_{4}\right] \\
\left(\bmod T^{(4)}\right) .
\end{gathered}
$$

Finalmente, como $\left[x_{1}, x_{2}, x_{7}\right]\left[x_{3}, x_{4}\right]\left[x_{5}, x_{6}\right] \equiv\left[x_{3}, x_{4}, x_{7}\right]\left[x_{1}, x_{2}\right]\left[x_{5}, x_{6}\right] \equiv\left[x_{5}, x_{6}, x_{7}\right]\left[x_{1}, x_{2}\right]\left[x_{3}, x_{4}\right]$ 
$\left(\bmod T^{(4)}\right)$ (Corolário 1.33$)$, segue de $(2-106)$ e $(2-110)-(2-112)$ que

$$
\left[q_{3}, x_{7}\right] \equiv 3 x_{1}^{2} x_{2}^{2} x_{3}^{2} x_{4}^{2} x_{5}^{2} x_{6}^{2}\left[x_{1}, x_{2}, x_{7}\right]\left[x_{3}, x_{4}\right]\left[x_{5}, x_{6}\right] \quad\left(\bmod T^{(4)}\right)
$$

Como $\operatorname{char}(F)=3$, segue que $q_{3}+T^{(4)}$ é central em $Q_{4}$.

Vamos mostrar agora que se $q_{n}+T^{(4)}$ é central em $Q_{4}$, então $n$ é divisível por 3. É suficiente mostrar que $q_{n}+T^{(4)}$ não é central em $Q_{4}$ quando $n=2 k+1$ ou $n=3 k+2$ para algum $k \in \mathbb{N}$. Por simplicidade, vamos considerar apenas o caso $n=3 k+2$ pois o caso $n=2 k+1$ é idêntico.

Seja $v_{n}=v_{n}\left(x_{1}, \ldots, x_{2 n}\right)=\left[x_{1}, x_{2}\right] \ldots\left[x_{2 n-1}, x_{2 n}\right]$. Observe que $v_{n}$ é uma componente multi-homogênea de $q_{n}\left(x_{1}+1, \ldots, x_{2 n}+1\right)$. Como $C\left(Q_{4}\right)$ é multi-homogêneo (Proposição 1.16) para mostrar que $q_{n}+T^{(4)}$ não é central em $Q_{4}$, é suficiente mostrar que $v_{n}+T^{(4)}$ não é central em $Q_{4}$.

Observe que $v_{3 k}=v_{3 k}\left(x_{1}, \ldots, x_{6 k}\right)$ e $v_{2}=v_{2}\left(x_{6 k+1}, x_{6 k+2}, x_{6 k+3}, x_{6 k+4}\right) \mathrm{com}$ $v_{n}=v_{3 k} v_{2}$. Usando a igualdade $[a b, c]=a[b, c]+[a, c] b$ e o fato de $v_{3 k}+T^{(4)}$ ser central em $Q_{4}$ (mostrado acima), temos

$$
\begin{aligned}
{\left[v_{n}, x_{6 k+5}\right] } & =\left[v_{3 k} v_{2}, x_{6 k+5}\right]=v_{3 k}\left[v_{2}, x_{6 k+5}\right]+\left[v_{3 k}, x_{6 k+5}\right] v_{2} \\
& \equiv v_{3 k}\left[v_{2}, x_{6 k+5}\right] \quad\left(\bmod T^{(4)}\right) .
\end{aligned}
$$

Como $v_{2}=\left[x_{6 k+1}, x_{6 k+2}\right]\left[x_{6 k+3}, x_{6 k+4}\right]$, usando novamente a igualdade $[a b, c]=a[b, c]+$ $[a, c] b$ e o fato de $\left[\left[x_{6 k+3}, x_{6 k+4}\right], x_{6 k+5}\right]+T^{(4)}$ ser central em $Q_{4}$, obtemos sucessivamente

$$
\begin{aligned}
{\left[v_{n}, x_{6 k+5}\right] \equiv v_{3 k}\left[v_{2}, x_{6 k+5}\right] } & \equiv v_{3 k}\left[\left[x_{6 k+1}, x_{6 k+2}\right]\left[x_{6 k+3}, x_{6 k+4}\right], x_{6 k+5}\right] \quad\left(\bmod T^{(4)}\right) \\
& \equiv v_{3 k}\left[x_{6 k+1}, x_{6 k+2}\right]\left[x_{6 k+3}, x_{6 k+4}, x_{6 k+5}\right]+ \\
& +v_{3 k}\left[x_{6 k+1}, x_{6 k+2}, x_{6 k+5}\right]\left[x_{6 k+3}, x_{6 k+4}\right] \quad\left(\bmod T^{(4)}\right) \\
& \equiv v_{3 k}\left[x_{6 k+3}, x_{6 k+4}, x_{6 k+5}\right]\left[x_{6 k+1}, x_{6 k+2}\right]+ \\
& +v_{3 k}\left[x_{6 k+1}, x_{6 k+2}, x_{6 k+5}\right]\left[x_{6 k+3}, x_{6 k+4}\right] \quad\left(\bmod T^{(4)}\right)
\end{aligned}
$$

Finalmente, pelo Corolário 1.33 obtemos

$$
\left[v_{n}, x_{6 k+5}\right] \equiv 2 v_{3 k}\left[x_{6 k+1}, x_{6 k+2}\right]\left[x_{6 k+3}, x_{6 k+4}, x_{6 k+5}\right] \quad\left(\bmod T^{(4)}\right)
$$

Como $\left[x_{6 k+1}, x_{6 k+2}\right]\left[x_{6 k+3}, x_{6 k+4}, x_{6 k+5}\right]+T^{(4)}$ é um elemento da base de $F\langle X\rangle / T^{(4)}$ (Lema 2.14), segue que $2 v_{3 k}\left[x_{6 k+1}, x_{6 k+2}\right]\left[x_{6 k+3}, x_{6 k+4}, x_{6 k+5}\right]$ não pertence a $T^{(4)}$. Portanto $\left[v_{n}, x_{6 k+5}\right]$ não pertence a $T^{(4)}$ e $v_{n}+T^{(4)}$ não é central em $Q_{4}$. Como vimos, isso implica que $q_{n}+T^{(4)}$ não é central em $Q_{4}$. O lema está demonstrado. 
Lema 2.28. Seja $F$ um corpo de característica 3. Então o polinômio $q_{n}$ pertence a $C\left(Q_{4}\right)+T^{(3)}$ se, e somente se $n$ é divisivel por 3 .

Demonstração. Se $n$ é divisível 3, já vimos no Lema 2.27 que $q_{n}$ pertence a $C\left(Q_{4}\right)$ e portanto $q_{n}$ pertence a $C\left(Q_{4}\right)+T^{(3)}$. Vamos mostrar agora que se $q_{n}$ pertence a $C\left(Q_{4}\right)+T^{(3)}$, então $n$ é divisível por 3. Suponha o contrário, que exista um número $n \in \mathbb{N}$ que não é divisível por 3 tal que $q_{n}$ pertença a $C\left(Q_{4}\right)+T^{(3)}$. Então existem $f \in C\left(Q_{4}\right)$ e $g \in T^{(3)}$ tais que

$$
f=q_{n}+g .
$$

Como $q_{n}$ possui multigrau $(3, \ldots, 3)$ e $C\left(Q_{4}\right)$ é multi-homogêneo (Proposição 1.16), podemos assumir que $g$ também possui multigrau $(3, \ldots, 3)$. Segue do Lema 2.24 que $g$ pertence ao $T$-subespaço gerado pelos polinômios (2-97)-(2-98). Como esses polinômios pertencem a $C\left(Q_{4}\right)$ (Lema 2.26), segue que $g$ pertence a $C\left(Q_{4}\right)$. Uma vez que $f=q_{n}+g$ com $f \in C\left(Q_{4}\right)$, segue que $q_{n}$ pertence a $C\left(Q_{4}\right)$, mas isso contraria o Lema 2.27 porque $n$ não é divisível por 3. Portanto, $q_{n} \in C\left(Q_{4}\right)+T^{(3)}$ implica $n$ divisível por 3. O lema está demonstrado.

Lema 2.29. Seja $F$ um corpo de característica 3. Então $\left[x_{1}, x_{2}\right]\left[x_{3}, x_{4}\right]-x_{1}\left[x_{2}, x_{3}, x_{4}\right]+$ $T^{(4)}$ é central na álgebra $Q_{4}=F\langle X\rangle / T^{(4)}$.

Demonstração. Pondo $f=\left[x_{1}, x_{2}\right]\left[x_{3}, x_{4}\right]-x_{1}\left[x_{2}, x_{3}, x_{4}\right]$ e usando a igualdade $[a b, c]=$ $a[b, c]+[a, c] b$, temos

$$
\left[f, x_{5}\right]=\left[x_{1}, x_{2}\right]\left[x_{3}, x_{4}, x_{5}\right]+\left[x_{1}, x_{2}, x_{5}\right]\left[x_{3}, x_{4}\right]-x_{1}\left[x_{2}, x_{3}, x_{4}, x_{5}\right]-\left[x_{1}, x_{5}\right]\left[x_{2}, x_{3}, x_{4}\right] .
$$

Como $\left[x_{1}, x_{2}\right]\left[x_{3}, x_{4}, x_{5}\right] \equiv\left[x_{3}, x_{4}, x_{5}\right]\left[x_{1}, x_{2}\right]\left(\bmod T^{(4)}\right), x_{1}\left[x_{2}, x_{3}, x_{4}, x_{5}\right] \in T^{(4)} \quad \mathrm{e}$ $\left[x_{1}, x_{5}\right]\left[x_{2}, x_{3}, x_{4}\right] \equiv\left[x_{2}, x_{3}, x_{4}\right]\left[x_{1}, x_{5}\right]\left(\bmod T^{(4)}\right)$, obtemos

$$
\left[f, x_{5}\right] \equiv\left[x_{3}, x_{4}, x_{5}\right]\left[x_{1}, x_{2}\right]+\left[x_{1}, x_{2}, x_{5}\right]\left[x_{3}, x_{4}\right]-\left[x_{2}, x_{3}, x_{4}\right]\left[x_{1}, x_{5}\right] \quad\left(\bmod T^{(4)}\right)
$$

Pelo Corolário 1.33 (ii) temos $\left[x_{3}, x_{4}, x_{5}\right]\left[x_{1}, x_{2}\right] \equiv\left[x_{1}, x_{2}, x_{5}\right]\left[x_{3}, x_{4}\right] \equiv-\left[x_{2}, x_{3}, x_{4}\right]\left[x_{1}, x_{5}\right]$ $\left(\bmod T^{(4)}\right)$. Assim

$$
\left[f, x_{5}\right] \equiv 3\left[x_{3}, x_{4}, x_{5}\right]\left[x_{1}, x_{2}\right] \quad\left(\bmod T^{(4)}\right)
$$

Como $\operatorname{char}(F)=3$, temos que $\left[f, x_{5}\right]$ pertence a $T^{(4)}$, isto é, $\left[x_{1}, x_{2}\right]-x_{1}\left[x_{2}, x_{3}, x_{4}\right]+T^{(4)}$ é central em $Q_{4}$.

Para uso na proposição seguinte, lembre que $M=\left\{x_{i_{1}} x_{i_{2}} \ldots x_{i_{n}} ; x_{i_{s}} \in X\right\}$ é o conjunto dos monômios mônicos de $F\langle X\rangle$. 
Proposição 2.30. Seja $F$ um corpo de característica 3. Então $\left(C\left(Q_{4}\right)+T^{(3)}\right) / T^{(3)}$ é gerado como espaço vetorial por $\left[a_{1}, a_{2}\right]\left[a_{3}, a_{4}\right]+T^{(3)}, a_{i} \in M$ e pelos elementos

$$
x_{i_{1}}^{3 r_{1}} \ldots x_{i_{k}}^{3 r_{k}} x_{j_{1}}^{2}\left[x_{j_{1}}, x_{j_{2}}\right] x_{j_{2}}^{2} \ldots x_{j_{2 l-1}}^{2}\left[x_{j_{2 l-1}}, x_{j_{2 l}}\right] x_{j_{2 l}}^{2}+T^{(3)}
$$

com $k \geq 0, r_{s}>0, l \equiv 0(\bmod 3), i_{1}<\ldots<i_{k}$ e $j_{1}<\ldots<j_{2 l}$

Demonstração. Seja $f=f\left(x_{1}, \ldots, x_{n}\right) \in C\left(Q_{4}\right)$ tal que $f \notin T^{(3)}$. Pela Proposição 1.16 podemos assumir que $f$ é um polinômio multi-homogêneo de grau $m_{i}$ em cada variável $x_{i}$. Suponha que algum $x_{i}$ não é divisível por 3. Reenumerando as variáveis $x_{i}$, podemos assumir, sem perda de generalidade que $i=1$. Pelo Lema 2.7 , temos que $f$ pertence ao $T$-subespaço gerado pelos polinômios $\left[x_{1}, x_{2}\right]\left[x_{3}, x_{4}\right]$ e $x_{1}\left[x_{2}, x_{3}, x_{4}\right]$. Consequentemente $f+T^{(3)}$ é uma combinação linear dos elementos $\left[a_{1}, a_{2}\right]\left[a_{3}, a_{4}\right]+T^{(3)}$ com $a_{i} \in M$. Como $\left[x_{1}, x_{2}\right]\left[x_{3}, x_{4}\right]-x_{1}\left[x_{2}, x_{3}, x_{4}\right]+T^{(4)}$ é central em $Q_{4}$ (Lema 2.29) e $x_{1}\left[x_{2}, x_{3}, x_{4}\right] \in T^{(3)}$, segue que os elementos $\left[a_{1}, a_{2}\right]\left[a_{3}, a_{4}\right]+T^{(3)}$ pertencem a $\left(C\left(Q_{4}\right)+T^{(3)}\right) / T^{(3)}$.

Agora, suponha que todos os $m_{i}$ sejam divisíveis por 3. Como $x_{0}^{3}+T^{(3)}$ e $\left[x_{1}, x_{2}\right]+T^{(3)}$ são centrais em $Q_{3}$, segue da Proposição 1.21 que $f+T^{(3)}$ é uma combinação linear de elementos da forma

$$
x_{i_{1}}^{3 r_{1}} \ldots x_{i_{k}}^{3 r_{k}} x_{j_{1}}^{2}\left[x_{j_{1}}, x_{j_{2}}\right] x_{j_{2}}^{2} \ldots x_{j_{2 l-1}}^{2}\left[x_{j_{2 l-1}}, x_{j_{2 l}}\right] x_{j_{2 l}}^{2}+T^{(3)}
$$

com $k, l \geq 0, r_{s}>0,1 \leq i_{1}<\ldots<i_{k} \leq n$ e $1 \leq j_{1}<\ldots<j_{2 l} \leq n$. Segue do Lema 2.27 que todo elemento da forma $(2-113) \operatorname{com} l \equiv 0(\bmod 3)$, pertence a $C\left(Q_{4}\right)+T^{(3)} / T^{(3)}$. Para concluir a demonstração resta mostrar que todo elemento (2-113) satisfaz $l \equiv 0$ $(\bmod 3)$. De fato, suponha que

$$
\alpha x_{i_{1}}^{3 r_{1}} \ldots x_{i_{k}}^{3 r_{k}} x_{j_{1}}^{2}\left[x_{j_{1}}, x_{j_{2}}\right] x_{j_{2}}^{2} \ldots x_{j_{2 l-1}}^{2}\left[x_{j_{2 l-1}}, x_{j_{2 l}}\right] x_{j_{2 l}}^{2}+T^{(3)}
$$

seja algum elemento de (2-113) com $\alpha \in F, \alpha \neq 0, l \not \equiv 0(\bmod 3)$ e $l$ minimal. Considere o endomorfismo $\varphi$ de $F\langle X\rangle$ tal que $\varphi\left(x_{j_{s}}\right)=x_{j_{s}}$, se $s \in\{1,2, \ldots, 2 l\}$ e $\varphi\left(x_{j_{s}}\right)=1$, se $s \notin\{1,2, \ldots 2 l\}$. Segue de (2-113) e (2-114) que

$$
\varphi(f)+T^{(3)}=\alpha x_{j_{1}}^{3 r_{1}} \ldots x_{j_{2 l}}^{3 r_{2 l}} q_{l}\left(x_{j_{1}}, \ldots, x_{j_{2 l}}\right)+T^{(3)} .
$$

Logo o polinômio $g\left(x_{j_{1}}, \ldots, x_{j_{2 l}}\right)=\alpha x_{j_{1}}^{3 r_{1}} \ldots x_{j_{2 l}}^{3 r_{2 l}} q_{l}$ pertence a $C\left(Q_{4}\right)+T^{(3)}$. Observe que $\alpha q_{l}$ é a componente multi-homogênea de multigrau $(3, \ldots, 3)$ do polinômio $g\left(x_{j_{1}}+1, \ldots, x_{j_{2 l}}+1\right)=\alpha\left(x_{j_{1}}+1\right)^{3 r_{1}} \ldots\left(x_{j_{2 l}}+1\right)^{3 r_{2 l}} q_{l}$. Como $g\left(x_{j_{1}}+1, \ldots, x_{j_{2 l}}+1\right)$ pertence a $C\left(Q_{4}\right)+T^{(3)}$ e $C\left(Q_{4}\right)+T^{(3)}$ é multi-homogêneo segue que $\alpha q_{l}$ pertence a $C\left(Q_{4}\right)+T^{(3)}$. Como $q_{l}$ não pertence a $C\left(Q_{4}\right)+T^{(3)}($ Lema 2.28$)$, temos $\alpha=0$, 
uma contradição. Portanto $l \equiv 0(\bmod 3)$ para todos os elementos em $(2-113)$, e isso conclui a demonstração.

\subsubsection{Os polinômios centrais da álgebra $Q_{4}$}

Nesta seção provaremos o Teorema 2.10, que é o primeiro resultado principal desta tese.

Lema 2.31. Seja $F$ um corpo de característica 3. Então $C\left(Q_{4}\right) /\left(C\left(Q_{4}\right) \cap T^{(3)}\right)$ é gerado como um espaço vetorial pelos elementos

(i) $\left[a_{1}, a_{2}\right]\left[a_{3}, a_{4}\right]-a_{1}\left[a_{2}, a_{3}, a_{4}\right]+\left(C\left(Q_{4}\right) \cap T^{(3)}\right), a_{i} \in M$,

(ii) $x_{i_{1}}^{3 r_{1}} \ldots x_{i_{k}}^{3 r_{k}} q_{l}\left(x_{j_{1}}, \ldots, x_{j_{2 l}}\right)+\left(C\left(Q_{4}\right) \cap T^{(3)}\right)$,

$k \geq 0, r_{s}>0, l \equiv 0(\bmod 3), i_{1}<\ldots<i_{k} e j_{1}<\ldots<j_{2 l}$.

Demonstração. Como $a_{1}\left[a_{2}, a_{3}, a_{4}\right]$ pertence a $T^{(3)}$, segue diretamente do Lema 2.30 que $\left(C\left(Q_{4}\right)+T^{(3)}\right) / T^{(3)}$ é gerado, como espaço vetorial, pelos elementos

$$
\begin{aligned}
& {\left[a_{1}, a_{2}\right]\left[a_{3}, a_{4}\right]-a_{1}\left[a_{2}, a_{3}, a_{4}\right]+T^{(3)}, a_{i} \in M,} \\
& x_{i_{1}}^{3 r_{1}} \ldots x_{i_{k}}^{3 r_{k}} q_{l}\left(x_{j_{1}}, \ldots, x_{j_{2 l}}\right)+T^{(3)}, \\
& k \geq 0, r_{s}>0, l \equiv 0 \quad(\bmod 3), i_{1}<\ldots<i_{k}, j_{1}<\ldots<j_{2 l} .
\end{aligned}
$$

Pelo Lema 2.29, todo representante $\left[a_{1}, a_{2}\right]\left[a_{3}, a_{4}\right]-a_{1}\left[a_{2}, a_{3}, a_{4}\right]$ de (2$115)$ pertence a $C\left(Q_{4}\right)$. Todo representante $x_{i_{1}}^{3 r_{1}} \ldots x_{i_{k}}^{3 r_{k}} q_{l}\left(x_{j_{1}}, \ldots, x_{j_{2 l}}\right)$ de $(2-116)$ também pertence a $C\left(Q_{4}\right)$. Isso segue diretamente do fato dos elementos $x_{0}^{3}+T^{(4)} \mathrm{e}$ $q_{l}\left(x_{j_{1}}, \ldots, x_{j_{2 l}}\right)+T^{(4)}$ serem centrais em $Q_{4}$ (Lema 1.22 e Lema 2.27 , respectivamente) e $C\left(Q_{4}\right)$ ser uma álgebra (Proposição 1.13).

É fácil ver que a aplicação $\varphi: f+T^{(3)} \rightarrow f+\left(C\left(Q_{4}\right) \cap T^{(3)}\right)$ é um isomorfismo entre os espaços vetoriais $A_{1}=\left(C\left(Q_{4}\right)+T^{(3)}\right) / T^{(3)}$ e $A_{2}=C\left(Q_{4}\right) /\left(C\left(Q_{4}\right) \cap T^{(3)}\right)$ (esse é um dos teoremas de isomorfismo). O isomorfismo $\varphi$ leva os elementos de (2-115)-(2116) (geradores de $\left.A_{1}\right)$ nos elementos (i)-(ii). Isso mostra que $C\left(Q_{4}\right) /\left(C\left(Q_{4}\right) \cap T^{(3)}\right)$ é gerado pelos elementos (i)-(ii).

Finalmente, podemos provar o teorema principal deste capítulo.

\section{Demonstração do Teorema 2.10}

Demonstração. Seja $f$ um polinômio central de $Q_{4}$. Pelo Lema 2.31, $f+\left(C\left(Q_{4}\right) \cap\right.$ $\left.T^{(3)}\right)=g+\left(C\left(Q_{4}\right) \cap T^{(3)}\right)$, onde $g$ é uma combinação linear dos polinômios

$$
\begin{aligned}
& {\left[a_{1}, a_{2}\right]\left[a_{3}, a_{4}\right]+a_{1}\left[a_{2}, a_{3}, a_{4}\right], a_{i} \in M,} \\
& x_{i_{1}}^{3 r_{1}} \ldots x_{i_{k}}^{3 r_{k}} q_{n}\left(x_{j_{1}}, \ldots, x_{j_{2 n}}\right), \\
& k \geq 0, r_{s}>0, l \equiv 0 \quad(\bmod 3), i_{1}<\ldots<i_{k}, j_{1}<\ldots<j_{2 l} .
\end{aligned}
$$


Sejam $q_{n}=q_{n}\left(x_{1}, \ldots, x_{2 n}\right)$ e $I=\left\langle x_{1}\left[x_{2}, x_{3}, x_{3}\right]\right\rangle^{T S}+\left\langle x_{1} \omega\left(x_{2}, x_{3}, x_{4}, x_{5}\right)\right\rangle^{T S}+T^{(4)}$. Segue do Lema 2.23 e do fato $I$ ser um ideal que

$$
\left(x_{1}^{3} \ldots x_{k}^{3}\right) q_{n} \equiv\left(x_{1} \ldots x_{k}\right)^{3} q_{n} \quad(\bmod I)
$$

Usando (2-117), (2-118) e (2-119) não é difícil verificar que $g$ pertence ao $T$-subespaço gerado pelos polinômios

$$
\begin{aligned}
& {\left[x_{1}, x_{2}\right]\left[x_{3}, x_{4}\right]-x_{1}\left[x_{2}, x_{3}, x_{4}\right], x_{1}\left[x_{2}, x_{3}, x_{3}\right], x_{1} \omega\left(x_{2}, x_{3}, x_{4}, x_{5}\right)} \\
& x_{1}\left[x_{2}, x_{3}, x_{4}, x_{5}\right], x_{0}^{3}, x_{0}^{3} q_{3}, x_{0}^{3} q_{6}, \ldots x_{0}^{3} q_{3 n}, \ldots
\end{aligned}
$$

Como $f-g$ pertence a $C\left(Q_{4}\right) \cap T^{(3)}$, segue da Proposição 2.26, que $f-g$ pertence ao $T$-subespaço gerado pelos polinômios

$$
\begin{aligned}
& {\left[x_{1}, x_{2}, x_{3}\right], x_{1}\left[x_{2}, x_{3}, x_{3}\right], x_{1} \omega\left(x_{2}, x_{3}, x_{4}, x_{5}\right), x_{1}\left[x_{2}, x_{3}, x_{4}, x_{5}\right],} \\
& x_{0}^{3} u_{0}, x_{0}^{3} u_{1}, x_{0}^{3} u_{2}, \ldots, x_{0}^{3} u_{n}, \ldots
\end{aligned}
$$

Assim, $f$ pertence ao $T$-subespaço gerado pelos polinômios (2-120)- (2-123). É fácil ver que os polinômios $\left[x_{1}, x_{2}, x_{3}\right]$ e $x_{1}\left[x_{2}, x_{3}, x_{3}\right]$ pertencem ao $T$-subespaço gerado pelo polinômio $\left[x_{1}, x_{2}\right]\left[x_{3}, x_{4}\right]-x_{1}\left[x_{2}, x_{3}, x_{4}\right]$. Portanto $f$ pertence ao $T$-subespaço gerado pelos polinômios de (i)-(iii). O Teorema está demonstrado.

Corolário 2.32. Seja $F$ um corpo de característica 3. Então o espaço vetorial $C\left(Q_{4}\right)$ dos polinômios centrais da álgebra $Q_{4}=F\langle X\rangle / T^{(4)}$ não é finitamente gerado como um $T$-subespaço de $F\langle X\rangle$.

Demonstração. Segue do Teorema 2.10 que $C\left(Q_{4}\right)+T^{(3)}$ é gerado como $T$-subespaço pelos polinômios

$$
x_{1}\left[x_{2}, x_{3}, x_{4}\right],\left[x_{1}, x_{2}\right]\left[x_{3}, x_{4}\right]-x_{1}\left[x_{2}, x_{3}, x_{4}\right], x_{0}^{3}, x_{0}^{3} q_{3}, x_{0}^{3} q_{6}, \ldots, x_{0}^{3} q_{3 n}, \ldots
$$

Seja $I$ o $T$-subespaço gerado pelos polinômios (2-124). Afirmamos que $I$ não é finitamente gerado como $T$-subespaço. A demonstração é idêntica àquela do Teorema 3 de [7]. Vamos fazer apenas um esboço. Para cada $n \in \mathbb{N}$, seja $V_{3 n}$ o $T$-subespaço gerado pelos polinômios $\left[x_{1}, x_{2}\right]\left[x_{3}, x_{4}\right]-x_{1}\left[x_{2}, x_{3}, x_{4}\right], q_{3}, q_{6}, \ldots, q_{3 n}$ e $W_{3 n}$ o $T$-subespaço gerado pelos polinômios $\left[x_{1}, x_{2}\right]\left[x_{3}, x_{4}\right]-x_{1}\left[x_{2}, x_{3}, x_{4}\right], x_{0}^{3}, x_{0}^{3} q_{3}, x_{0}^{3} q_{6}, \ldots, x_{0}^{3} q_{3 n}$. Pode ser mostrado de forma análoga à demonstração do Teorema 3 de [7] que a cadeia

$$
W_{3}+T^{(3)} \subseteq W_{6}+T^{(3)} \subseteq \ldots \subseteq W_{3 n}+T^{(3)} \subseteq \ldots
$$

contém uma subcadeia estritamente ascendente. Como $I=\bigcup_{n}\left(W_{3 n}+T^{(3)}\right), I$ não é 
finitamente gerado como $T$-subespaço . Assim $C\left(Q_{4}\right)+T^{(3)}$ não é finitamente gerado como $T$-subespaço e consequentemente $C\left(Q_{4}\right)$ não é finitamente gerado como $T$ subespaço. 


\section{Polinômios hipercentrais}

Seja $A$ uma álgebra sobre um corpo $F$. Sejam $f=f\left(x_{1}, \ldots, x_{n}\right) \in F\langle X\rangle$ um polinômio e $y_{1}, \ldots, y_{m} \in X$ com $\left\{x_{1}, \ldots, x_{n}\right\} \cap\left\{y_{1}, \ldots, y_{m}\right\}=\emptyset$. Dizemos que $f$ é um polinômio $m$-central de $A$ se $\left[f, y_{1}, \ldots, y_{m}\right]$ pertence a $\operatorname{Id}(A)$. O conjunto de todos os polinômios $m$-centrais de $A$ será denotado por

$$
C_{m}(A)=\left\{f=f\left(x_{1}, \ldots, x_{n}\right) \in F\langle X\rangle:\left[f, y_{1}, \ldots, y_{m}\right] \in I d(A)\right\}
$$

Observe que para cada $m \in \mathbb{N}$, o conjunto $C_{m}(A)$ forma um subespaço vetorial de $F\langle X\rangle$. Observe também que $C_{1}(A)=C(A)$, isto é, os polinômios 1-centrais são os polinômios centrais. Como todo polinômio $m$-central é também $(m+1)$-central, se pusermos $C_{0}(A)=\{0\}$, obtemos a seguinte cadeia de inclusões

$$
\{0\}=C_{0}(A) \subseteq C(A)=C_{1}(A) \subseteq C_{2}(A) \subseteq \ldots \subseteq C_{m-1}(A) \subseteq C_{m}(A) \subseteq \ldots
$$

que chamaremos de série central superior da álgebra $A$. Os polinômios $m$-centrais de $A$ com $m>1$ são chamados genericamente de polinômios hipercentrais de $A$.

Observe que $C_{1}\left(Q_{3}\right)=C\left(Q_{3}\right)$ já foi descrito (Proposição 1.28, Corolário 1.25) e é imediato verificar que $C_{2}\left(Q_{3}\right)=F\langle X\rangle$. Observe também que $C_{1}\left(Q_{4}\right)=C\left(Q_{4}\right)$ já foi descrito (Proposição 2.6, Teorema 2.1, Teorema 2.10, Teorema 0.2).

Neste capítulo, vamos dar uma descrição dos polinômios hipercentrais das álgebras $Q_{4}=F\langle X\rangle / T^{(4)}$ e $Q_{5}=F\langle X\rangle / T^{(5)}$.

Proposição 3.1. $C_{m}(A)$ é um $T$-subespaço de $F\langle X\rangle$.

Demonstração. Seja $f=f\left(x_{1}, \ldots, x_{n}\right)$ um polinômio $m$-central de $A$ e $\varphi$ um endomorfismo qualquer de $F\langle X\rangle$. Devemos mostrar que $\varphi(f)$ é um polinômio $m$-central de $A$. De fato, como

$$
\left[\varphi\left(f\left(x_{1}, \ldots, x_{n}\right)\right), y_{1}, \ldots, y_{m}\right]=\left[f\left(\varphi\left(x_{1}\right), \ldots, f\left(\varphi\left(x_{n}\right)\right), y_{1}, \ldots, y_{m}\right]\right.
$$


e $\left[f\left(x_{1}, \ldots, x_{n}\right), y_{1}, \ldots, y_{m}\right] \in \operatorname{Id}(A)$, o polinômio do lado direito da igualdade (3-1) pertence a $\operatorname{Id}(A), \log \mathrm{\varphi}(f)$ é um polinômio $m$-central de $A$.

Quando $m \geq 2$, a equivalência seguinte decorre imediatamente da definição de polinômio $m$-central:

$f$ pertence a $C_{m}(A)$ se, e somente se $\left[f, y_{1}\right]$ pertence a $C_{m-1}(A)$.

Proposição 3.2. $C_{m}(A)$ é uma $T$-subálgebra de $F\langle X\rangle$.

Demonstração. Como $C_{m}(A)$ é um $T$-subespaço de $F\langle X\rangle$ (Proposição 3.1), é suficiente mostrar que $C_{m}(A)$ é uma subálgebra de $F\langle X\rangle$. Sejam $f$ e $g$ polinômios de $C_{m}(A)$, devemos mostrar que $f g$ pertence a $C_{m}(A)$. Segue da igualdade $[a b, c]+[b c, a]+[c a, b]=0$ que

$$
\left[f g, y_{1}\right]=\left[f, g y_{1}\right]+\left[g, y_{1} f\right]
$$

Como $\left[f, y_{2}\right]$ e $\left[g, y_{3}\right]$ pertencem a $C_{m-1}(A)$ e $C_{m-1}(A)$ é um $T$-subespaço, fazendo $y_{2}=g y_{1}$ e $y_{3}=y_{1} f$ segue que $\left[f, g y_{1}\right]$ e $\left[g, y_{1} f\right]$ pertencem a $C_{m-1}(A)$. Logo $\left[f g, y_{1}\right]$ pertence a $C_{m-1}$ e portanto $f g$ pertence a $C_{m}(A)$.

Proposição 3.3. Seja $F$ um corpo qualquer. Então o $T$-subespaço $C_{m}\left(Q_{n}\right)$ é multihomogêneo.

Demonstração. Inteiramente análoga a demonstração da Proposição 1.16.

\subsection{A álgebra $Q_{4}$}

Nesta seção vamos dar uma descrição dos polinômios hipercentrais da álgebra $Q_{4}=F\langle X\rangle / T^{(4)}$. Mais precisamente, se $\operatorname{char}(F)=p$, vamos mostrar que

$$
\begin{aligned}
& C_{2}\left(Q_{4}\right)=\left\langle x_{0}^{p}\right\rangle^{T S}+T^{(2)}, \text { se } p=2 \text { ou } p>3 \\
& C_{2}\left(Q_{4}\right)=F .1+T^{(2)}, \text { se } p=0 \\
& C_{2}\left(Q_{4}\right)=C\left(Q_{3}\right), \text { se } p=3 .
\end{aligned}
$$

Lema 3.4. Seja $F$ um corpo de característica $\neq 3$. Então $T^{(2)} \subseteq C_{2}\left(Q_{4}\right)$.

Demonstração. Pela Proposição 1.17 (i),

$$
\begin{aligned}
{\left[x_{1}\left[x_{2}, x_{3}\right], x_{4}, x_{5}\right] } & =x_{1}\left[x_{2}, x_{3}, x_{4}, x_{5}\right]+\left[x_{1}, x_{4}\right]\left[x_{2}, x_{3}, x_{5}\right]+\left[x_{1}, x_{5}\right]\left[x_{2}, x_{3}, x_{4}\right]+ \\
& +\left[x_{1}, x_{4}, x_{5}\right]\left[x_{2}, x_{3}\right] .
\end{aligned}
$$


É claro que $x_{1}\left[x_{2}, x_{3}, x_{4}, x_{5}\right]$ pertence a $T^{(4)}$. Pelo Lema 1.33 (iii), os polinômios $\left[x_{1}, x_{4}\right]\left[x_{2}, x_{3}, x_{5}\right],\left[x_{1}, x_{5}\right]\left[x_{2}, x_{3}, x_{4}\right]$ e $\left[x_{1}, x_{4}, x_{5}\right]\left[x_{2}, x_{3}\right]$ também pertencem a $T^{(4)}$. Assim $x_{1}\left[x_{2}, x_{3}\right]$ é um polinômio 2 -central de $Q_{4}$. Como $T^{(2)}$ é gerado como $T$-subespaço por $x_{1}\left[x_{2}, x_{3}\right]$ e $C_{2}\left(Q_{4}\right)$ é um $T$-subespaço (Proposição 3.1), temos $T^{(2)} \subseteq C_{2}\left(Q_{4}\right)$.

Lema 3.5. Seja $F$ um corpo de característica $p \geq 2$. Então $x_{0}^{p}$ é um polinômio 2-central de $Q_{4}$.

Demonstração. Suponha primeiramente que $p=2$. Como

$$
\left[x_{0}^{2}, y_{1}\right]=x_{0}\left[x_{0}, y_{1}\right]+\left[x_{0}, y_{1}\right] x_{0}=2 x_{0}\left[x_{0}, y_{1}\right]+\left[x_{0}, y_{1}, x_{0}\right]=\left[x_{0}, y_{1}, x_{0}\right]
$$

segue que $\left[x_{0}^{2}, y_{1}, y_{2}\right]$ pertence a $T^{(4)}$, isto é, $x_{0}^{2}$ é um polinômio 2-central de $Q_{4}$. Agora se $p=3$ ou $p>3$, então segue do Lema 2.20 e Lema 1.22 (i), respectivamente, que $x_{0}^{p}$ é um polinômio central de $Q_{4}$, consequentemente $x_{0}^{p}$ é um polinômio 2-central de $Q_{4}$.

Proposição 3.6. Seja $F$ um corpo de característica $p=2$ ou $p>3$. Então $C_{2}\left(Q_{4}\right)=$ $\left\langle x_{0}^{p}\right\rangle^{T S}+T^{(2)}$.

Demonstração. Vamos mostrar primeiramente a inclusão $\left\langle x_{0}^{p}\right\rangle^{T S}+T^{(2)} \subseteq C_{2}\left(Q_{4}\right)$. Como $x_{0}^{p}$ é um polinômio 2-central de $Q_{4}$ (Lema 3.5), $T^{(2)} \subseteq C_{2}\left(Q_{4}\right)$ (Lema 3.4) e $C_{2}\left(Q_{4}\right)$ é um $T$-subespaço (Proposição 3.1), segue a inclusão $\left\langle x_{0}^{p}\right\rangle^{T S}+T^{(2)} \subseteq C_{2}\left(Q_{4}\right)$.

Vejamos a inclusão contrária. Seja $f=f\left(x_{1}, \ldots, x_{n}\right)$ um polinômio 2-central de $Q_{4}$ com $f \notin T^{(2)}$. Pela Proposição 3.3, podemos assumir, sem perda de generalidade, que $f$ é multi-homogêneo de grau $m_{i}$ em cada variávei $x_{i}$. Assim

$$
f+T^{(2)}=\alpha x_{1}^{m_{1}} \ldots x_{n}^{m_{n}}+T^{(2)}, 0 \neq \alpha \in F .
$$

Suponha que algum $m_{i}$ não seja divisível por $p$ e defina o endomorfismo $\varphi$ de $F\langle X\rangle$ por $\varphi\left(x_{i}\right)=x_{i}$ e $\varphi\left(x_{s}\right)=1$ se $s \neq i$. Por (3-2), temos $\varphi(f)+T^{(2)}=\alpha x_{i}^{m_{i}}+T^{(2)}$.

Como $T^{(2)} \subseteq C_{2}\left(Q_{4}\right)$, temos que $x_{i}^{m_{i}}$ é um polinômio 2-central de $Q_{4}$. Sendo $C_{2}\left(Q_{4}\right)$ um $T$-subespaço, segue que $g=\left(x_{i}+1\right)^{m_{i}}$ é um polinômio 2-central de $Q_{4}$. Como $m_{i} x_{i}$ é uma componente multi-homogênea de $g$ e $C_{2}\left(Q_{4}\right)$ é multi-homogêneo, segue que $m_{i} x_{i}$ é um polinômio 2-central de $Q_{4}$. Como $m_{i} \neq 0$, segue que $x_{i}$ é um polinômio 2-central de $Q_{4}$, uma contradição. Assim todos os graus $m_{i}$ de (32) são múltiplos de $p$ e portanto $f$ pertence a $\left\langle x_{0}^{p}\right\rangle^{T S}+T^{(2)}$. Isso mostra que $C_{2}\left(Q_{4}\right) \subseteq\left\langle x_{0}^{p}\right\rangle^{T S}+T^{(2)}$.

Proposição 3.7. Seja $F$ um corpo de característica 0 , então $C_{2}\left(Q_{4}\right)=F .1+T^{(2)}$. 
Demonstração. Pelo Lema 3.4, temos $T^{(2)} \subseteq C_{2}\left(Q_{4}\right)$. Vejamos a inclusão contrária. Seja $f=f\left(x_{1}, \ldots, x_{n}\right)$ um polinômio 2-central de $Q_{4}$. Pela Proposição 1.9 não há perda de generalidade em supor que $f$ é multilinear. Assim, existe $\alpha \in F$ tal que

$$
f+T^{(2)}=\alpha x_{1} \ldots x_{n}+T^{(2)}
$$

Como $T^{(2)} \subseteq C_{2}\left(Q_{4}\right)$, segue de (3-3) que $g\left(x_{1}, \ldots, x_{n}\right)=\alpha x_{1} \ldots x_{n}$ é um polinômio 2-central de $Q_{4}$. Sendo $C_{2}\left(Q_{4}\right)$ um $T$-subespaço (Proposição 3.1), segue que $g\left(x_{1}, 1, \ldots, 1\right)=\alpha x_{1}$ é também um polinômio 2-central de $Q_{4}$. Uma vez que $x_{1}$ não é um polinômio 2-central de $Q_{4}$, temos $\alpha=0$; e segue de (3-3) que $f$ pertence a $T^{(2)}$. Portanto $C_{2}\left(Q_{4}\right) \subseteq T^{(2)}$, e a proposição está demonstrada.

Lema 3.8. Seja $F$ um corpo qualquer. Então $T^{(3)} \subseteq C_{2}\left(Q_{4}\right)$.

Demonstração. Pela Proposição 1.17 (i), temos

$$
\begin{aligned}
{\left[x_{1}\left[x_{2}, x_{3}, x_{4}\right], y_{1}, y_{2}\right] } & =x_{1}\left[x_{2}, x_{3}, x_{4}, y_{1}, y_{2}\right]+\left[x_{1}, y_{1}\right]\left[x_{2}, x_{3}, x_{4}, y_{2}\right]+ \\
& +\left[x_{1}, y_{2}\right]\left[x_{2}, x_{3}, x_{4}, y_{1}\right]+\left[x_{1}, y_{1}, y_{2}\right]\left[x_{2}, x_{3}, x_{4}\right]
\end{aligned}
$$

É claro que os polinômios $x_{1}\left[x_{2}, x_{3}, x_{4}, y_{1}, y_{2}\right],\left[x_{1}, y_{1}\right]\left[x_{2}, x_{3}, x_{4}, y_{2}\right]$ e $\left[x_{1}, y_{2}\right]\left[x_{2}, x_{3}, x_{4}, y_{1}\right]$ pertencem a $T^{(4)}$. Como o polinômio $\left[x_{1}, y_{1}, y_{2}\right]\left[x_{2}, x_{3}, x_{4}\right]$ também pertence a $T^{(4)}$ (Lema 1.32), segue que $\left[x_{1}\left[x_{2}, x_{3}, x_{4}\right], y_{1}, y_{2}\right]$ pertence a $T^{(4)}$. Assim $x_{1}\left[x_{2}, x_{3}, x_{4}\right]$ é um polinômio 2-central de $Q_{4}$. Como $T^{(3)}$ é gerado como $T$-subespaço pelo polinômio $x_{1}\left[x_{2}, x_{3}, x_{4}\right]$ e $C_{2}\left(Q_{4}\right)$ é um $T$-subespaço (Proposição 3.1), obtemos $T^{(3)} \subseteq C_{2}\left(Q_{4}\right)$.

Lema 3.9. Seja $F$ um corpo de característica $p>0$. Então $q\left(x_{1}, x_{2}\right)=$ $x_{1}^{p-1}\left[x_{1}, x_{2}\right] x_{2}^{p-1}$ é um polinômio 2-central de $Q_{4}$.

Demonstração. Usando a igualdade $\left[a_{1} a_{2} \ldots a_{n}, a\right]=\sum_{i=1}^{n} a_{1} \ldots a_{i-1}\left[a_{i}, a\right] a_{i+1} \ldots a_{n}$ (Lema 1.17 (ii)), obtemos

$$
\begin{aligned}
{\left[x_{1}^{p-1}\left[x_{1}, x_{2}\right] x_{2}^{p-1}, x_{3}, x_{4}\right] } & =\left[\left[x_{1}, x_{3}\right] x_{1}^{p-2}\left[x_{1}, x_{2}\right] x_{2}^{p-1}, x_{4}\right]+\ldots+ \\
& +\left[x_{1}^{p-1}\left[\left[x_{1}, x_{2}\right], x_{3}\right] x_{2}^{p-1}, x_{4}\right]+\ldots+ \\
& +\left[x_{1}^{p-1}\left[x_{1}, x_{2}\right] x_{2}^{p-2}\left[x_{2}, x_{3}\right], x_{4}\right] .
\end{aligned}
$$

Usando o Lema 1.17 (ii) em cada parcela do lado direito da igualdade (3-4) e usando também o Corolário 1.33 (i)-(ii), não é difícil mostrar que $\left[\left[x_{1}^{p-1}\left[x_{1}, x_{2}\right] x_{2}^{p-1}, x_{3}, x_{4}\right] \equiv 0\right.$ $\left(\bmod T^{(4)}\right)$, isto é, $q\left(x_{1}, x_{2}\right)=x_{1}^{p-1}\left[x_{1}, x_{2}\right] x_{2}^{p-1}$ é um polinômio 2 -central de $Q_{4}$.

Lema 3.10. Seja $F$ um corpo qualquer. Então $C\left(Q_{3}\right) \subseteq C_{2}\left(Q_{4}\right)$. 
Demonstração. Vamos dividir a demonstração em dois casos: $\operatorname{char}(F)=0$ e $\operatorname{char}(F)=p>0$. Seja $\operatorname{char}(F)=0$. Pela Proposição 1.28, $C\left(Q_{3}\right)$ é gerado como $T$-subespaço por 1 e pelos polinômios $\left[x_{1}, x_{2}\right]$ e $x_{1}\left[x_{2}, x_{3}, x_{4}\right]$. É claro que $\left[x_{1}, x_{2}\right]$ é um polinômio 2-central de $Q_{4}$. Pelo Lema 3.8 temos que $x_{1}\left[x_{2}, x_{3}, x_{4}\right]$ é um polinômio 2-central de $Q_{4}$. Portanto $C\left(Q_{3}\right) \subseteq C_{2}\left(Q_{4}\right)$.

Agora seja $\operatorname{char}(F)=p>0$. Pelo Corolário 1.25, $C\left(Q_{3}\right)$ é gerado como uma $T$-subálgebra, pelos polinômios $x_{1}\left[x_{2}, x_{3}, x_{4}\right], x_{0}^{3}$ e $q\left(x_{1}, x_{2}\right)=x_{1}^{p-1}\left[x_{1}, x_{2}\right] x_{2}^{p-1}$. Como $C_{2}\left(Q_{4}\right)$ é também uma $T$-subálgebra (Proposição 3.2), é suficiente mostrar que esses geradores pertencem $C_{2}\left(Q_{4}\right)$. Já sabemos que $x_{1}\left[x_{2}, x_{3}, x_{4}\right]$ é um polinômio 2-central de $Q_{4}$. Pelo Lema 3.5, $x_{0}^{p}$ é um polinômio 2-central de $\left.Q_{4}\right)$ e pelo Lema $3.9, q\left(x_{1}, x_{2}\right)$ também é um polinômio 2-central de $Q_{4}$. Isso mostra que $C\left(Q_{3}\right) \subseteq C_{2}\left(Q_{4}\right)$ também no caso $p \geq 2$. O lema está demonstrado.

O lema seguinte é bem conhecido.

Lema 3.11 ([20], Lema 12). Sejam $F$ um corpo qualquer e $f=f\left(x_{1}, \ldots, x_{n}\right) \in F\langle X\rangle$ um polinômio multi-homogêneo de grau 1 em $x_{1}$. Seja $L=\langle f\rangle^{T S}+\left\langle\left[x_{1}, x_{2}\right]\right\rangle^{T S}+$ $T^{(3)}$. Então $L=F\langle X\rangle$ ou $L=\left\langle\left[x_{1}, x_{2}\right]\right\rangle^{T S}+T^{(3)}$ ou $L=\left\langle x_{1}\left[x_{2}, x_{3}\right] \ldots\left[x_{2 s}, x_{2 s+1}\right]\right\rangle^{T S}+$ $\left\langle\left[x_{1}, x_{2}\right]\right\rangle^{T S}+T^{(3)}$ para algum $s \leq(n-1) / 2$.

Demonstração. Veja a referência [20], páginas 15 e 16.

Lema 3.12. Sejam $F$ um corpo de característica 3 e $f=f\left(x_{1}, \ldots, x_{n}\right) \in F\langle X\rangle$ um polinômio homogêneo de grau 1 em $x_{1}$. Se $f$ é 2-central em $Q_{4}=F\langle X\rangle / T^{(4)}$, então $f \in\left\langle\left[x_{1}, x_{2}\right]\right\rangle^{T S}+T^{(3)}$.

Demonstração. Seja $L=\langle f\rangle^{T S}+\left\langle\left[x_{1}, x_{2}\right]\right\rangle^{T S}+T^{(3)}$. Pelo Lema 3.11 ocorre uma das três possibilidades:

(i) $L=F\langle X\rangle$,

(ii) $L=\left\langle\left[x_{1}, x_{2}\right]\right\rangle^{T S}+T^{(3)}$,

(iii) $L=\left\langle x_{1}\left[x_{2}, x_{3}\right] \ldots\left[x_{2 s}, x_{2 s+1}\right]\right\rangle^{T S}+\left\langle\left[x_{1}, x_{2}\right]\right\rangle^{T S}+T^{(3)}$.

É claro que $\left[x_{1}, x_{2}\right]$ é um polinômio 2-central de $Q_{4}$. Pelo Lema 3.8, temos $T^{(3)} \subseteq$ $C_{2}\left(Q_{4}\right)$. Assim $L \subseteq C_{2}\left(Q_{4}\right)$. Se ocorrer (i), então $C_{2}\left(Q_{4}\right)=F\langle X\rangle$, o que não é possível. Se ocorrer (iii), então o polinômio $x_{1}\left[x_{2}, x_{3}\right] \ldots\left[x_{2 s}, x_{2 s+1}\right]$ é 2-central em $Q_{4}$. Observe que

$$
\begin{aligned}
{\left[x_{3}\left[x_{4}, x_{5}\right] \ldots\left[x_{2(s+1)}, x_{2(s+1)+1}\right],\left[x_{1}, x_{2}\right]\right] } & =x_{3}\left[\left[x_{4}, x_{5}\right] \ldots\left[x_{2(s+1)}, x_{2(s+1)+1}\right],\left[x_{1}, x_{2}\right]\right]+ \\
& +\left[x_{1}, x_{2}, x_{3}\right]\left[x_{4}, x_{5}\right] \ldots\left[x_{2(s+1)}, x_{2(s+1)+1}\right](3-5)
\end{aligned}
$$


Como $C_{2}\left(Q_{4}\right)$ é um $T$-subespaço (Proposição 3.1), segue que o polinômio $\left[x_{3}\left[x_{4}, x_{5}\right] \ldots\left[x_{2(s+1)}, x_{2(s+1)+1}\right],\left[x_{1}, x_{2}\right]\right]$ pertence a $T^{(4)}$. Usando a igualdade $[a b, c]=$ $a[b, c]+[a, c] b$ e a relação $\left[\left[x_{1}, x_{2}\right],\left[x_{3}, x_{4}\right]\right]=\left[x_{1}, x_{2}, x_{3}, x_{4}\right]-\left[x_{1}, x_{2}, x_{4}, x_{3}\right] \in T^{(4)}$ não é difícil ver que o polinômio $x_{3}\left[\left[x_{4}, x_{5}\right] \ldots\left[x_{2(s+1)}, x_{2(s+1)+1}\right],\left[x_{1}, x_{2}\right]\right]$ também pertence a $T^{(4)}$. Portanto, segue de (3-5) que o polinômio $\left[x_{1}, x_{2}, x_{3}\right]\left[x_{4}, x_{5}\right] \ldots\left[x_{2(s+1)}, x_{2(s+1)+1}\right]$ pertence a $T^{(4)}$, o que é uma contradição com a Proposição 2.14.

Portanto ocorre o caso (ii), isto é $L=\left\langle\left[x_{1}, x_{2}\right]\right\rangle^{T S}+T^{(3)}$. Isso mostra que $f$ pertence a $\left\langle\left[x_{1}, x_{2}\right]\right\rangle^{T S}+T^{(3)}$.

A demonstração do lema a seguir é idêntica à demonstração do Lema 1.29. Iremos escrevê-la para conveniência do leitor.

Lema 3.13. Sejam $F$ um corpo de característica 3 e $f=f\left(x_{1}, \ldots, x_{n}\right) \in F\langle X\rangle$ um polinômio homogêneo de grau $m_{1}$ em $x_{1}$, onde $m_{1}$ não é divisível por 3 . Se $f$ for 2-central em $Q_{4}$, então $f \in\left\langle\left[x_{1}, x_{2}\right]\right\rangle^{T S}+T^{(3)}$.

Demonstração. Pela Proposição 3.3, não há perda de generalidade em supor que $f$ é multi-homogêneo de grau $m_{1}$ em $x_{1}$. Escreva $m_{1}=3 q+r$ com $0<r<3$. Usando a base de $Q_{3}$ dada na Proposição 1.21, vemos que existe $g=g\left(x_{1}, \ldots, x_{n}\right) \in F\langle X\rangle$, multi-homogêneo de grau $r$ em $x_{1}$ tal que

$$
f+T^{(3)}=x_{1}^{3 q} g+T^{(3)}
$$

Defina o endomorfismo $\varphi$ de $F\langle X\rangle$ por $\varphi\left(x_{1}\right)=1+x_{1}$ e $\varphi\left(x_{i}\right)=1$ se $i \neq 1$. Por (3-6) temos

$$
\varphi(f)+T^{(3)}=\left(1+x_{1}^{3}\right)^{q} g\left(1+x_{1}, x_{2}, \ldots, x_{n}\right)+T^{(3)} .
$$

Como $g\left(x_{1}, \ldots, x_{n}\right)$ é a componente multi-homogênea de grau $r$ em $x_{1}$ do polinômio $\left(1+x_{1}^{p}\right)^{q} g\left(1+x_{1}, x_{2}, \ldots, x_{n}\right), C_{2}\left(Q_{4}\right)$ é um $T$-subespaço multi-homogêneo e $T^{(3)} \subseteq C_{2}\left(Q_{4}\right)$ (Lema 3.8), segue de (3-7) que $g$ é 2-central em $Q_{4}$. Seja $h=$ $h\left(y_{1}, \ldots, y_{r}, x_{2}, \ldots, x_{n}\right)$ a linearização total de $g$ em $x_{1}$. Então $h$ é 2-central em $Q_{4}$ e segue do Lema 3.12 que $h \in\left\langle\left[x_{1}, x_{2}\right]\right\rangle^{T S}+T^{(3)}$. Como

$$
h\left(x_{1}, \ldots, x_{1}, x_{2}, \ldots, x_{n}\right)=r ! g\left(x_{1}, \ldots, x_{n}\right),
$$

obtemos $g \in\left\langle\left[x_{1}, x_{2}\right]\right\rangle^{T S}+T^{(3)}$. Como $x_{1}^{3}+T^{(3)}$ é central em $Q_{3}$ (Lema $\left.1.22(\mathrm{i})\right)$ e $C\left(Q_{3}\right)$ é uma álgebra (Proposição 1.13) segue que $x_{1}^{p q}+T^{(3)}$ é central em $Q_{3}$. Assim

$$
x_{1}^{p q}\left[x_{2}, x_{3}\right]+T^{(3)}=\left[x_{1}^{p q} x_{2}, x_{3}\right]+T^{(3)} .
$$

Como $g \in\left\langle\left[x_{1}, x_{2}\right]\right\rangle^{T S}+T^{(3)}$ segue de (3-6) e (3-8) que $f \in\left\langle\left[x_{1}, x_{2}\right]\right\rangle^{T S}+T^{(3)}$. 
Proposição 3.14. Seja $F$ um corpo de característica 3. Então $C_{2}\left(Q_{4}\right)=C\left(Q_{3}\right)$.

Demonstração. Pelo Lema 3.10, temos $C\left(Q_{3}\right) \subseteq C_{2}\left(Q_{4}\right)$. Vamos mostrar que $C_{2}\left(Q_{4}\right) \subseteq C\left(Q_{3}\right)$. Seja $f=f\left(x_{1}, \ldots, x_{n}\right)$ um polinômio 2-central de $Q_{4}$. Pela Proposição 3.1 , podemos assumir que $f$ é multi-homogêneo com multigrau $m_{i}$ em cada variável $x_{i}$.

Suponha que algum $m_{i}$ não seja divisível por 3. Reenumerando as variáveis $x_{i}$, podemos assumir, sem perda de generalidade que $i=1$. Pelo Lema 3.12, temos $f \in\left\langle\left[x_{1}, x_{2}\right]\right\rangle^{T S}+T^{(3)}$. Como $\left[x_{1}, x_{2}\right]+T^{(3)}$ é central em $Q_{3}$ e $T^{(3)} \subseteq C\left(Q_{3}\right)$ segue que $f+T^{(3)}$ é central em $Q_{3}$.

Suponha agora que todos os $m_{i}$ sejam divisíveis por 3 . Como $x_{0}^{3}+T^{(3)} \mathrm{e}$ $\left[x_{1}, x_{2}\right]+T^{(3)}$ são centrais em $Q_{3}$, segue da Proposição 1.21 que $f+T^{(3)}$ é uma combinação linear de elementos da forma

$$
x_{i_{1}}^{3 r_{1}} \ldots x_{i_{k}}^{3 r_{k}} x_{j_{1}}^{2}\left[x_{j_{1}} x_{j_{2}}\right] x_{j_{2}}^{2} \ldots x_{j_{2 l-1}}^{2}\left[x_{j_{2 l-1}}, x_{j_{2 l} l}\right] x_{j_{2 l}}^{2}+T^{(3)}
$$

onde $k, l \geq 0, r_{s}>0,1 \leq i_{1}<\ldots<i_{k} \leq n, 1 \leq j_{1}<\ldots<j_{2 l} \leq n$. Pelo Teorema 1.24 todos os elementos da forma (3-9) são centrais em $Q_{3}$, logo $f+T^{(3)}$ é central em $Q_{3}$. Mostramos assim que $C_{2}\left(Q_{4}\right) \subseteq C\left(Q_{3}\right)$.

\subsection{A álgebra $Q_{5}$}

Nesta seção vamos dar uma descrição dos polinômios hipercentrais da álgebra $Q_{5}=F\langle X\rangle / T^{(5)}$. Mais precisamente, se $\operatorname{char}(F)=p$, vamos mostrar que

$$
\begin{aligned}
& C_{2}\left(Q_{5}\right)=\left\langle x_{0}^{p}\right\rangle^{T S}+T^{(2)} \text { se } p>2 \\
& C_{2}\left(Q_{5}\right)=F .1+T^{(3)} \text { se } p=0 ; \\
& C_{3}\left(Q_{5}\right)=C\left(Q_{3}\right) \text { para } p \geq 0 .
\end{aligned}
$$

O lema seguinte é bem conhecido, veja [12, Lema 2], [14, página 16] e [43, Corolário 4].

Lema $3.15([12,14,43])$. Seja $F$ um corpo de característica $\neq 2$. Então $T^{(5)} \subseteq$ $\operatorname{Id}\left(E \otimes E_{2}\right)$.

Demonstração. Devemos mostrar que a álgebra $E \otimes E_{2}$ satisfaz a identidade $\left[x_{1}, x_{2}, x_{3}, x_{4}, x_{5}\right]=0$. Observe que $E$ e $E_{2}$ possuem bases

$$
\begin{aligned}
& 1, e_{i_{1}} e_{i_{2}} \ldots e_{i_{n}}, i_{1}<i_{2}<\ldots<i_{n}, n \in \mathbb{N} \\
& 1, h_{1}, h_{2}, h_{1} h_{2}
\end{aligned}
$$


respectivamente. Como o polinômio $\left[x_{1}, x_{2}, x_{3}, x_{4}, x_{5}\right]$ é multilinear, é suficiente avaliálo na base de $E \otimes E_{2}$, isto é, nos elementos $a \otimes b$, onde $a$ é um elemento de (3-10) e $b$ é um elemento de (3-11). Sejam $a_{1}, \ldots, a_{5}$ elementos quaisquer de $E$ e $b_{1}, \ldots, b_{5}$ elementos quaisquer de $E_{2}$. Observe que

$$
\begin{aligned}
{\left[a_{1} \otimes b_{1}, a_{2} \otimes b_{2}\right] } & =a_{1} a_{2} \otimes b_{1} b_{2}-a_{2} a_{1} \otimes b_{2} b_{1} \\
& =a_{1} a_{2} \otimes b_{1} b_{2}-a_{2} a_{1} \otimes b_{1} b_{2}+a_{2} a_{1} \otimes b_{1} b_{2}-a_{2} a_{1} \otimes b_{2} b_{1} \\
& =\left[a_{1}, a_{2}\right] \otimes b_{1} b_{2}+a_{2} a_{1} \otimes\left[b_{1}, b_{2}\right] .
\end{aligned}
$$

Usando (3-12) três vezes e observando que $\left[x_{1}, x_{2}\right]$ é um polinômio central tanto de $E$ quanto de $E_{2}$, podemos obter

$$
\begin{aligned}
{\left[a_{1} \otimes b_{1}, a_{2} \otimes b_{2}, a_{3} \otimes b_{3}, a_{4} \otimes b_{4}, a_{5} \otimes b_{5}\right] } & =\left[a_{1}, a_{2}\right]\left[a_{3}, a_{4}\right] a_{5} \otimes b_{1}\left[b_{2}, b_{3}\right]\left[b_{4}, b_{5}\right]+ \\
& +\left[a_{1}, a_{2}\right]\left[a_{3}, a_{4}\right] a_{5} \otimes b_{2}\left[b_{1}, b_{3}\right]\left[b_{4}, b_{5}\right]+ \\
& +\left[a_{2} a_{1}, a_{3}\right]\left[a_{4}, a_{5}\right] \otimes\left[b_{1}, b_{2}\right]\left[b_{3}, b_{4}\right] b_{5} .
\end{aligned}
$$

Como a base de $E_{2}$ é formada pelos elementos (3-11), é fácil ver que $E_{2}$ satisfaz a identidade polinomial $\left[x_{1}, x_{2}\right]\left[x_{3}, x_{4}\right]=0$. Assim $\left[a_{1} \otimes b_{1}, a_{2} \otimes b_{2}, a_{3} \otimes b_{3}, a_{4} \otimes b_{4}, a_{5} \otimes b_{5}\right]=0$ para quaisquer elementos $a_{1}, \ldots a_{5}$ de (3-10) e $b_{1}, \ldots, b_{5}$ de (3-11). Consequentemente $\left[x_{1}, x_{2}, x_{3}, x_{4}, x_{5}\right]=0$ é uma identidade polinomial para a álgebra $E \otimes E_{2}$.

Lema 3.16. Seja $F$ um corpo qualquer. Então $T^{(3)} \subseteq C_{2}\left(Q_{5}\right)$.

Demonstração. Pelo Lema 1.17 (i), temos

$$
\begin{aligned}
{\left[x_{1}\left[x_{2}, x_{3}, x_{4}\right], x_{5}, x_{6}\right] } & =x_{1}\left[x_{2}, x_{3}, x_{4}, x_{5}, x_{6}\right]+\left[x_{1}, x_{5}\right]\left[x_{2}, x_{3}, x_{4}, x_{6}\right]+ \\
& +\left[x_{1}, x_{6}\right]\left[x_{2}, x_{3}, x_{4}, x_{5}\right]+\left[x_{1}, x_{5}, x_{6}\right]\left[x_{2}, x_{3}, x_{4}\right] \\
& \equiv x_{1}\left[x_{2}, x_{3}, x_{4}, x_{5}, x_{6}\right]+\left[x_{2}, x_{3}, x_{4}, x_{6}\right]\left[x_{1}, x_{5}\right]+ \\
& +\left[x_{2}, x_{3}, x_{4}, x_{5}\right]\left[x_{1}, x_{6}\right]+\left[x_{1}, x_{5}, x_{6}\right]\left[x_{2}, x_{3}, x_{4}\right]\left(\bmod T^{(5)}\right) .
\end{aligned}
$$

É bem conhecido que $\left[x_{2}, x_{3}, x_{4}, x_{6}\right]\left[x_{1}, x_{5}\right]+\left[x_{2}, x_{3}, x_{4}, x_{5}\right]\left[x_{1}, x_{6}\right] \in T^{(5)}$ (veja [[9], Teorema 1.1] e [[26], Proposição 1]). Também é bem conhecido que $\left[x_{1}, x_{5}, x_{6}\right]\left[x_{2}, x_{3}, x_{4}\right] \in$ $T^{(5)}$ (veja [[9], Teorema 1.1], [[44],Lema 1]). Portanto, segue de (3-13) que $\left[x_{1}\left[x_{2}, x_{3}, x_{4}\right], x_{5}, x_{6}\right]$ pertence a $T^{(5)}$. Como $T^{(3)}$ é gerado como um $T$-subespaço pelo polinômio $x_{1}\left[x_{2}, x_{3}, x_{4}\right]$ e $C_{2}\left(Q_{5}\right)$ é um $T$-subespaço (Proposição 1.13 ), temos $T^{(3)} \subseteq C_{2}\left(Q_{5}\right)$.

Lema 3.17. Seja $F$ um corpo de característica $\neq 2$. Então para qualquer $n \in \mathbb{N}$, $\left[x_{1}, x_{2}\right] \ldots\left[x_{2 n-1}, x_{2 n}\right]$ não é um polinômio 2-central de $Q_{5}$. 
Demonstração. Dado $n \in \mathbb{N}$ qualquer, seja $v_{n}=v_{n}\left(x_{1}, \ldots, x_{2 n}\right)=\left[x_{1}, x_{2}\right] \ldots\left[x_{2 n-1}, x_{2 n}\right]$. Fazendo as substituições $x_{1}=e_{1} \otimes h_{1}, x_{2}=e_{2} \otimes 1, \ldots, x_{2 n-1}=e_{2 n-1} \otimes 1, x_{2 n}=e_{2 n} \otimes$ $1, y_{1}=e_{2 n+1} \otimes h_{2}, y_{2}=e_{2 n+2} \otimes 1$ no polinômio

$$
\left[v_{n}\left(x_{1}, x_{2}, \ldots, x_{2 n}\right), y_{1}, y_{2}\right]
$$

obtemos $2^{n+2} e_{1} \ldots e_{2 n+2} \otimes h_{1} h_{2}$, que não é nulo porque é um múltiplo escalar de um elemento da base de $E \otimes E_{2}$. Assim $\left[v_{n}, y_{1}, y_{2}\right]$ não pertence a $\operatorname{Id}\left(G \otimes G_{2}\right)$. Como $T^{(5)} \subseteq I d\left(G \otimes G_{2}\right)$ (Lema 3.15), segue que $\left[v_{n}, y_{1}, y_{2}\right]$ não pertence a $T^{(5)}$, isto é $v_{n}$ não é um polinômio 2-central de $Q_{5}$.

Lema 3.18. Seja $F$ um corpo de característica $p>2$. Então $x_{0}^{p}$ é um polinômio 2-central de $Q_{5}=F\langle X\rangle / T^{(5)}$.

Demonstração. Como vimos na demonstração do Lema 2.20

$$
\left[x_{0}^{3}, x_{1}\right]=3 x_{0}^{2}\left[x_{0}, x_{1}\right]+3 x_{0}\left[x_{0}, x_{1}, x_{0}\right]+\left[x_{0}, x_{1}, x_{0}, x_{0}\right]
$$

Se $p=3$, temos $\left[x_{0}^{3}, x_{1}, x_{2}\right]=\left[x_{0}, x_{1}, x_{0}, x_{0}, x_{2}\right]$. Assim $x_{0}^{3}$ é um polinômio 2-central de $Q_{5}$. Agora se $p>3$, então segue do Lema 1.22 (i) que $x_{0}^{p}$ é um polinômio 2-central de $Q_{5}$.

Proposição 3.19. Seja $F$ um corpo de característica $p>2$. Então $C_{2}\left(Q_{5}\right)=$ $\left\langle x_{0}^{p}\right\rangle^{T S}+T^{(3)}$.

Demonstração. Pelo Lema 3.18, $x_{0}^{p}$ é um polinômio 2-central de $Q_{5}$. Como $T^{(3)} \subseteq$ $C_{2}\left(Q_{5}\right)$ (Lema 3.16) e $C_{2}\left(Q_{5}\right)$ é um $T$-subespaço (Proposição 3.1), segue que $\left\langle x_{0}^{p}\right\rangle^{T S}+T^{(3)} \subseteq C_{2}\left(Q_{5}\right)$.

Vamos mostrar a inclusão contrária. Seja $f=f\left(x_{1}, \ldots, x_{n}\right)$ um polinômio 2-central de $Q_{5}$ com $f \notin T^{(3)}$. Pela Proposição 3.2 podemos supor, sem perda de generalidade, que $f$ é multi-homogêneo. Como $\left[x_{1}, x_{2}\right]+T^{(3)}$ é central em $Q_{3}$, segue da Proposição 1.21 que $f+T^{(3)}$ é uma combinação linear de elementos da forma

$$
x_{i_{1}}^{r_{1}} \ldots x_{i_{k}}^{r_{k}}\left[x_{j_{1}}, x_{j_{2}}\right] \ldots\left[x_{j_{2 l-1}}, x_{j_{2 l}}\right]+T^{(3)}
$$

com $k \geq 0, l \geq 0, r_{s}>0,1 \leq i_{1}<\ldots<i_{k} \leq n, 1 \leq j_{1}<\ldots j_{2 l} \leq n$.

Afirmamos que $l=0$ para todos os elementos de (3-14), isto é, não aparecem comutadores. Suponha o contrário e seja $g_{l, j_{1}}+T^{(3)}$ o termo de (3-14) com $l$ e $j_{1}$ minimais. Seja $\varphi$ o endomorfismo de $F\langle X\rangle$ definido por $\varphi\left(x_{j_{s}}\right)=x_{j_{s}}$ se $s \in\{1, \ldots, 2 l\}$ e $\varphi\left(x_{s}\right)=1$ se $s \notin\left\{j_{1}, \ldots, j_{2 l}\right\}$. Por (3-14) temos

$$
\varphi(f)+T^{(3)}=x_{j_{1}}^{s_{1}} \ldots x_{j_{2 l}}^{s_{2 l}}\left[x_{j_{1}}, x_{j_{2}}\right] \ldots\left[x_{j_{2 l-1}}, x_{j_{2 l}}\right]+T^{(3)}
$$


$\operatorname{Seja} h\left(x_{j_{1}}, \ldots, x_{j_{2 l}}\right)=x_{j_{1}}^{r_{1}} \ldots x_{j_{2 l}}^{r_{2 l}}\left[x_{j_{1}}, x_{j_{2}}\right] \ldots\left[x_{j_{2 l-1}}, x_{j_{2 l}}\right]$. Observe que $\left[x_{j_{1}}, x_{j_{2}}\right] \ldots\left[x_{j_{2 l-1}}, x_{j_{2 l}}\right]$ é uma componente multi-homogênea de $h\left(x_{j_{1}}+1, \ldots, x_{j_{2 l}}+1\right)$. Como $T^{(3)} \subseteq C_{2}\left(Q_{5}\right)$ e $C\left(Q_{5}\right)$ é multi-homogêneo, segue de (3-15) que $\left[x_{j_{1}}, x_{j_{2}}\right] \ldots\left[x_{j_{2 l-1}}, x_{j_{2 l}}\right]$ é um polinômio 2-central de $Q_{5}$. Mas isso contraria o Lema 3.17. Logo $l=0$ em cada elemento (3-14) e assim $f+T^{(3)}$ é um múltiplo escalar do elemento

$$
x_{1}^{r_{1}} \ldots x_{n}^{r_{n}}+T^{(3)} .
$$

Afirmamos que cada grau $r_{i}, i=1, \ldots, n$ é divisível por $p$. Suponha o contrário, que algum $r_{t}$ não seja divisível por $p$. Defina o endomorfismo $\phi$ de $F\langle X\rangle$ por $\phi\left(x_{t}\right)=x_{t}$ e $\phi\left(x_{s}\right)=1$ se $s \neq t$. Então segue de (3-16) que

$$
\phi(f)+T^{(3)}=x_{t}^{r_{t}}+T^{(3)} .
$$

Como $T^{(3)} \subseteq C_{2}\left(Q_{5}\right)$, segue de (3-17) que $x_{t}^{r_{t}}$ é um polinômio 2-central de $Q_{5}$. Como $C_{2}\left(Q_{5}\right)$ é um $T$-subespaço, segue que $g=\left(x_{t}+1\right)^{r_{t}}$ é um polinômio 2-central de $Q_{5}$. Como $r_{t} x_{t}$ é uma componente multi-homogênea de $g$ e $C_{2}\left(Q_{5}\right)$ é multi-homogêneo (Proposição 3.1), segue que $r_{t} x_{t}$ é um polinômio 2-central de $Q_{5}$. Isso só é possível se $r_{t}=0$, o que contraria a escolha de $r_{t}$.

Assim todos os graus $r_{i}, i=1, \ldots, n$ são divisíveis por $p$ e por (3-16), temos

$$
f+T^{(3)}=x_{1}^{p q_{1}} \ldots x_{n}^{p q_{n}}+T^{(3)} .
$$

Como $\left(x_{1} x_{2}\right)^{p} \equiv x_{1}^{p} x_{2}^{p}\left(\bmod T^{(3)}\right)\left(\right.$ Lema $1.22($ ii) $)$, segue de $(3-18)$ que $f+T^{(3)}=$ $\left(x_{1}^{q_{1}} \ldots x_{n}^{q_{n}}\right)^{p}+T^{(3)}$. Pondo $x_{0}=x_{1}^{q_{1}} \ldots x_{n}^{q_{n}}$, obtemos $f \in\left\langle x_{0}^{p}\right\rangle^{T S}+T^{(3)}$. Portanto $C_{2}\left(Q_{5}\right) \subseteq\left\langle x_{0}^{p}\right\rangle^{T S}+T^{(3)}$.

Proposição 3.20. Seja $F$ um corpo de característica 0 . Então $C_{2}\left(Q_{5}\right)=T^{(3)}$.

Demonstração. Pelo Lema 3.16 temos $T^{(3)} \subseteq C_{2}\left(Q_{5}\right)$. Vejamos a inclusão contrária. Seja $f=f\left(x_{1}, \ldots, x_{n}\right)$ um polinômio 2-central de $Q_{5}$. Conforme mostrado na Proposição 3.19:

$$
f+T^{(3)}=\alpha x_{1} \ldots x_{n}+T^{(3)},
$$

para algum $\alpha \in F$. Como $T^{(3)} \subseteq C_{2}\left(Q_{5}\right)$, segue de (3-19) que $\alpha x_{1} \ldots x_{n}$ é um polinômio 2-central de $Q_{5}$. Defina o endomorfismo $\varphi$ de $F\langle X\rangle$ por $\varphi\left(x_{1}\right)=x_{1}$ e $\varphi\left(x_{s}\right)=1$ se $s \neq 1$. Então $\varphi\left(x_{1} \ldots x_{n}\right)=\alpha x_{1}$ é um polinômio 2-central de $Q_{5}$. Mas isso implica $\alpha=0$ porque todo polinômio 2-central de $Q_{5}$ possui grau no mínimo 3 . Consequentemente $C_{2}\left(Q_{4}\right) \subseteq T^{(3)}$, o que prova a proposição. 
Lema 3.21. Seja $F$ um corpo de característica $\neq 2$. Então para qualquer $n \in \mathbb{N}$, $x_{1}\left[x_{2}, x_{3}\right] \ldots\left[x_{2 n}, x_{2 n+1}\right]$ não é um polinômio 3-central de $Q_{5}$.

Demonstração. Dado $n \in \mathbb{N}$ qualquer seja $v_{n}=v_{n}\left(x_{2}, \ldots, x_{2 n+1}\right)=$ $\left[x_{2}, x_{3}\right] \ldots\left[x_{2 n}, x_{2 n+1}\right]$. Fazendo as substituições $x_{1}=e_{1} \otimes 1, x_{2}=e_{2} \otimes 1, x_{3}=$ $e_{3} \otimes 1, \ldots, x_{2 n}=e_{2 n} \otimes 1, x_{2 n+1}=e_{2 n+1} \otimes 1, y_{1}=e_{2 n+2} \otimes h_{1}, y_{2}=e_{2 n+3} \otimes h_{2}, y_{3}=e_{2 n+4} \otimes 1$ no polinômio

$$
\left[x_{1} v_{n}\left(x_{2}, x_{3}, \ldots, x_{2 n+1}\right), y_{1}, y_{2}, y_{3}\right]
$$

obtemos $2^{n+3} e_{1} \ldots e_{2 n+4} \oplus h_{1} h_{2}$, que não é nulo porque é um múltiplo escalar de um elemento da base de $G \otimes G_{2}$. Assim $\left[x_{1} v_{n}, y_{1}, y_{2}, y_{3}\right]$ não pertence a $I d\left(G \otimes G_{2}\right)$. Como $T^{(5)} \subseteq I d\left(G \otimes G_{2}\right)$ (Lema 3.15), segue que $\left[x_{1} v_{n}, y_{1}, y_{2}, y_{3}\right]$ não pertence a $T^{(5)}$, isto é $x_{1} v_{n}$ não é um polinômio 3-central de $Q_{5}$.

Lema 3.22. Sejam $F$ um corpo de característica $\neq 2$ e $f=f\left(x_{1}, \ldots, x_{n}\right) \in F\langle X\rangle$ um polinômio homogêneo de grau 1 em $x_{1}$. Se f for 3-central em $Q_{5}=F\langle X\rangle / T^{(5)}$, então $f \in\left\langle\left[x_{1}, x_{2}\right]\right\rangle+T^{(3)}$.

Demonstração. Seja $L=\langle f\rangle^{T S}+\left\langle\left[x_{1}, x_{2}\right]\right\rangle^{T S}+T^{(3)}$. Pelo Lema 3.11 ocorre uma das três possibilidades:

(i) $L=F\langle X\rangle$,

(ii) $L=\left\langle\left[x_{1}, x_{2}\right]\right\rangle^{T S}+T^{(3)}$,

(iii) $L=\left\langle x_{1}\left[x_{2}, x_{3}\right] \ldots\left[x_{2 s}, x_{2 s+1}\right]\right\rangle^{T S}+\left\langle\left[x_{1}, x_{2}\right]\right\rangle^{T S}+T^{(3)}$.

É claro que $\left[x_{1}, x_{2}\right]$ é um polinômio 3-central de $Q_{5}$. Pelo Lema 3.16, temos $T^{(3)} \subseteq$ $C_{3}\left(Q_{5}\right)$. Assim $L \subseteq C_{3}\left(Q_{5}\right)$. Se ocorrer (i), então $C_{3}\left(Q_{5}\right)=F\langle X\rangle$, o que não é possível. Se ocorrer (iii), então $x_{1}\left[x_{2}, x_{3}\right] \ldots\left[x_{2 s}, x_{2 s+1}\right]$ é um polinômio 3 -central de $Q_{5}$, o que contraria o Lema 3.21. Portanto deve ocorrer (ii): $L=\left\langle\left[x_{1}, x_{2}\right]\right\rangle^{T S}+T^{(3)}$; e isso implica $f \in\left\langle\left[x_{1}, x_{2}\right]\right\rangle^{T S}+T^{(3)}$.

Proposição 3.23. Seja $F$ um corpo de característica 0 . Então $C_{3}\left(Q_{5}\right)=\left\langle\left[x_{1}, x_{2}\right]\right\rangle^{T S}+$ $T^{(3)}$.

Demonstração. Como $C_{3}\left(Q_{5}\right)$ é um $T$-subespaço (Proposição 3.1), segue da Proposição 1.9 que $C_{3}\left(Q_{5}\right)$ é gerado como $T$-subespaço por seus polinômios multi-lineares. Esses geradores multilineares são, em particular, homogêneos de grau 1 em $x_{1}$ e pelo Lema 3.22, pertencem a $\left\langle\left[x_{1}, x_{2}\right]\right\rangle^{T S}+T^{(3)}$. Assim $C_{3}\left(Q_{5}\right) \subseteq\left\langle\left[x_{1}, x_{2}\right]\right\rangle^{T S}+T^{(3)}$. Vejamos a inclusão contrária. É claro que $\left[x_{1}, x_{2}\right]$ é um polinômio 3-central de $Q_{5}$. Como $T^{(3)} \subseteq C_{2}\left(Q_{5}\right)$ (Lema 3.16) e $C_{2}\left(Q_{5}\right) \subseteq C_{3}\left(Q_{5}\right)$, temos $T^{(3)} \subseteq C_{3}\left(Q_{5}\right)$. Sendo $C_{3}\left(Q_{5}\right)$ um $T$-subespaço, segue imediatamente que $\left\langle\left[x_{1}, x_{2}\right]\right\rangle^{T S}+T^{(3)} \subseteq C_{3}\left(Q_{5}\right)$. 
Proposição 3.24. Seja $F$ um corpo qualquer. Então $C_{3}\left(Q_{5}\right)=C\left(Q_{3}\right)$.

Demonstração. Se $\operatorname{char}(F)=0$, então segue da Proposição 1.28 e da Proposição 3.23 que

$$
C\left(Q_{3}\right)=\left\langle\left[x_{1}, x_{2}\right]\right\rangle^{T S}+T^{(3)}=C_{3}\left(Q_{5}\right) .
$$

Podemos então fixar o caso $\operatorname{char}(F)=p>0$. Vamos mostrar primeiramente que $C\left(Q_{3}\right) \subseteq C_{3}\left(Q_{5}\right)$. Como $C\left(Q_{3}\right)$ é gerada como $T$-subálgebra por $x_{1}\left[x_{2}, x_{3}, x_{4}\right], x_{0}^{p}$ e $x_{1}^{p-1}\left[x_{1}, x_{2}\right] x_{2}^{p-1}$ (Corolário 1.25), é suficiente mostrar eles são polinômios 3-centrais de $Q_{5}$. Como $x_{1}\left[x_{2}, x_{3}, x_{4}\right]$ é um polinômio 2-central de $Q_{5}$ (Lema 3.16), é claro que ele é um polinômio 3-central de $Q_{5}$. Observe que

$$
\left[x_{0}^{2}, y_{1}\right]=2 x_{0}\left[x_{0}, y_{1}\right]+\left[x_{0}, y_{1}, x_{0}\right]
$$

Assim, se $p=2$, temos $\left[x_{0}^{2}, y_{1}, y_{2}, y_{3}\right]=\left[x_{0}, y_{1}, x_{0}, y_{2}, y_{3}\right] \in T^{(5)}$, isto é $x_{0}^{2}$ é um polinômio 3-central de $Q_{5}$. Se $p>2$, então segue do Lema 3.18 que $x_{0}^{p}$ é um polinômio 2-central de $Q_{5}$; logo ele é também um polinômio 3-central de $Q_{5}$.

Finalmente observe que $\left[x_{1}^{p-1}\left[x_{1}, x_{2}\right] x_{2}^{p-1}, y_{1}\right] \in T^{(3)}$ (Lema 1.30). Como $T^{(3)} \subseteq C_{2}\left(Q_{5}\right)$, segue imediatamente que $x_{1}^{p-1}\left[x_{1}, x_{2}\right] x_{2}^{p-1}$ é um polinômio 3-central de $Q_{5}$. Portanto $C\left(Q_{5}\right) \subseteq C_{3}\left(Q_{5}\right)$.

Vejamos a inclusão contrária. Seja $f=f\left(x_{1}, \ldots, x_{n}\right)$ um polinômio 3-central de $Q_{5}$. Pela Proposição 3.3, $C_{3}\left(Q_{5}\right)$ é gerado como $T$-subespaço por seus polinômios multi-homogêneos. Assim podemos considerar $f$ multi-homogêneo de grau $m_{i}$ em cada variável $x_{i}, i=1, \ldots, n$.

Suponha que algum grau $m_{i}$ não seja divisível por $p$. Sem perda de generalidade, podemos considerar $i=1$. Prosseguindo de modo inteiramente análogo ao Lema 3.13, podemos mostrar que $f$ pertence a $\left\langle\left[x_{1}, x_{2}\right]\right\rangle^{T S}+T^{(3)}$. Como $\left\langle\left[x_{1}, x_{2}\right]\right\rangle^{T S}+T^{(3)} \subseteq C\left(Q_{3}\right)$, segue que $f$ é um polinômio central de $Q_{3}$.

Agora suponha que cada $m_{i}, i=1, \ldots, n$ seja divisível por $p$. Como $\left[x_{1}, x_{2}\right]+$ $T^{(3)}$ e $x_{0}^{p}+T^{(3)}$ são centrais em $Q_{3}=F\langle X\rangle / T^{(3)}$. Segue da Proposição 1.21 que $f+T^{(3)}$ é uma combinação linear de elementos da forma

$$
\begin{aligned}
& x_{i_{1}}^{p r_{1}} \ldots x_{i_{k}}^{p r_{k}} x_{j_{1}}^{p-1}\left[x_{j_{1}}, x_{j_{2}}\right] x_{j_{2}}^{p-1} \ldots x_{j_{2 l-1}}^{p-1}\left[x_{j_{2 l-1}}, x_{j_{2 l} l}\right] x_{j_{2 l}}^{p-1}+T^{(3)}, \\
& k \geq 0, l \geq 1, r_{s}>0,1 \leq i_{1}<\ldots<i_{k} \leq n, 1 \leq j_{1}<\ldots<j_{k} \leq n .
\end{aligned}
$$

Segue imediatamente do Teorema 1.24 que $f+T^{(3)}$ é central em $Q_{3}$. Mostramos assim que $C_{3}\left(Q_{5}\right) \subseteq C\left(Q_{3}\right)$, o que demonstra a proposição. 


\subsection{Resumo dos resultados}

Reunimos abaixo uma descrição dos polinômios centrais e hipercentrais das álgebras que foram apresentadas nessa tese; os itens (4)-(5) e (7)-(12) foram obtidos por nós. O número $p$ denota a característica do corpo $F$.

1. $C\left(Q_{3}\right)=\left\langle\left[x_{1}, x_{2}\right]\right\rangle^{T S}+T^{(3)}$, se $p=0$;

2. $C\left(Q_{3}\right)=\left\langle x_{0}^{p}, x_{0}^{p} q_{1}, x_{0}^{p} q_{2}, \ldots\right\rangle^{T S}+T^{(3)}$, se $p \geq 2$;

3. $C\left(Q_{4}\right)=F .1+\left\langle\left[x_{1}, x_{2}\right]\left[x_{3}, x_{4}\right]\right\rangle^{T S}+T^{(4)}$, se $p=0$;

4. $C\left(Q_{4}\right)=\left\langle x_{0}^{4}, x_{0}^{2} q_{2}, x_{0}^{2} q_{3}, \ldots\right\rangle^{T S}$, se $p=2$;

5. $C\left(Q_{4}\right)=\left\langle x_{1} \omega\left(x_{2}, x_{3}, x_{4}, x_{5}\right),\left[x_{1}, x_{2}\right]\left[x_{3}, x_{4}\right]-x_{1}\left[x_{2}, x_{3}, x_{4}\right]\right\rangle^{T S}+$ $+\left\langle x_{0}^{3}, x_{0}^{3} q_{3}, x_{0}^{3} q_{6}, \ldots, x_{0}^{3} q_{3 n}, \ldots\right\rangle^{T S}+$ $+\left\langle x_{0}^{3} u_{0}, x_{0}^{3} u_{1}, x_{0}^{3} u_{2}, \ldots, x_{0}^{3} u_{n}, \ldots\right\rangle^{T S}+T^{(4)}$, se $p=3$;

6. $C\left(Q_{4}\right)=\left\langle x_{0}^{2} q_{2}, x_{0}^{2} q_{3}, \ldots\right\rangle^{T S}+T^{(4)}$, se $p>3$;

7. $C_{2}\left(Q_{4}\right)=F .1+T^{(2)}$, se $p=0$;

8. $C_{2}\left(Q_{4}\right)=\left\langle x_{0}^{p}\right\rangle^{T S}+T^{(2)}$, se $p=2$ ou $p>3$;

9. $C_{2}\left(Q_{4}\right)=C\left(Q_{3}\right)$, se $p=3$;

10. $C_{2}\left(Q_{5}\right)=F .1+T^{(3)}$, se $p=0$;

11. $C_{2}\left(Q_{5}\right)=\left\langle x_{0}^{p}\right\rangle^{T S}+T^{(2)}$, se $p>2$;

12. $C_{3}\left(Q_{5}\right)=C\left(Q_{3}\right)$ para $p \geq 0$.

Recentemente, uma lista de polinômios centrais para a álgebra $Q_{n}=$ $F\langle X\rangle / T^{(n)}$ para diversos valores de $n$ foi encontrada por Grishin e Pchelintsev [25]. Uma descrição completa dos polinômios centrais da álgebra $Q_{n}$ quando $n \geq 5$, permanece desconhecida. 


\section{Referências Bibliográficas}

[1] S.A. Amitsur, J. Levitzki, Minimal identities for algebras, Proceedings of the American Mathematical Society 1, 449-463 (1950).

[2] S. Bhupatiraju, P. Etingof, D. Jordan, W. Kuszmaul, J. Li, Lower central series of a free associative algebra over the integers and finite fields, Journal of Algebra 372, 251-274 (2012).

[3] C. Bekh-Ochir, S.A. Rankin, The central polinomials of the infinite dimensional unitary and nonunitary Grassmann algebras, Journal of Algebra and its Applications 9, 235-249 (2010).

[4] C. Bekh-Ochir, S.A.Rankin, Examples of associative algebras for which the T-space of central polynomials is not finitely based, Israel Journal of Mathematics 186, 333-347 (2011).

[5] C. Bekh-Ochir, D. Riley, On the Grassmann T-space, Journal of Algebra and its Applications 7, 319-336 (2008).

[6] A. Ya. Belov, On non-Specht varieties, Fundamentalnaya i Prikladnaya Matematika 5, 47-66 (1999).

[7] A. Brandão Jr., P. Koshlukov, A. Krasilnikov, E. A. Silva, The central polynomials for the Grassmann algebra, Israel Journal of Mathematics 179, 127-144 (2010).

[8] J. Colombo, P. Koshlukov, Central polynomials in the matrix algebra of order two, Linear Algebra and its Applications 377, 53-67 (2004).

[9] E. A. Costa and A. Krasilnikov, Relations in universal Lie nilpotent associative algebras of class 4, arXiv: 1306.429.

[10] G. Deryabina, A. Krasilnikov, The subalgebra of graded central polynomials of an associative algebra, Journal of Algebra 425, 313-323 (2015).

[11] G. Deryabina, A. Krasilnikov, The torsion subgroup of the additive group of a Lie nilpotent associative ring of class 3, Journal of Algebra 428, 230-255 (2015). 
[12] G. Deryabina, A. Krasilnikov, Products of commutators in a Lie nilpotent associative algebra, arXiv: 1509.08890 .

[13] V. Drensky, Free Algebras and PI-Algebras, Graduate Course in Algebra, Springer, Singapore (1999).

[14] V. Drensky, Codimensions of $T$-ideals and Hilbert series of relatively free algebras, Journal of Algebra 91, 1-17 (1984).

[15] V. Drensky and E. Formanek, Polynomial identity rings. Advanced Courses in Mathematics, CRM Barcelona, Birkhauser (2012).

[16] P. Etingof, J. Kim, X. Ma, On universal Lie nilpotent associative algebras, Journal of Algebra 321, 697-703 (2009).

[17] E. Formanek, Central polynomials for matrix rings, Journal of Algebra 23, 129132 (1972).

[18] A. Giambruno and P. Koshlukov, On the identities of the Grassmann algebras in characteristic $p>0$, Israel Journal of Mathematics 122, 305-316 (2001).

[19] A. Giambruno, M. Zaicev, Polynomial Identities and Asymptotic Methods, Mathematical Surveys and Monographs, 122, American Mathematical Society, Providence, Providence, RI, (2015).

[20] D. J. Gonçalves, A. Krasilnikov, I. Sviridova, Limit $T$-spaces and central polynomials in $n$ variables of the Grassmann algebra, Journal of Algebra 371, 156-174 (2012).

[21] A.S. Gordienko, Codimensions of commutators of lenght 4, Russian Mathematical Surveys 62, 187-188 (2007).

[22] A. V. Grishin, On the center of a relatively free Lie-nilpotent algebra of index 4, Mathematical Notes 91, no. 1, 139-140 (2012).

[23] A. V. Grishin, On the structure of the center of a relatively free Grassmann algebra, Russian Mathematical Surveys 65, 781-782 (2010).

[24] A. V. Grishin, Examples of $T$-spaces and $T$-ideals of characteristic 2 without the finite basis property, Fundamentalnaya i Prikladnaya Matematika 5 101-118 (1999).

[25] A.V. Grishin, S.V. Pchelintsev, On centers of relatively free associative algebras with a Lie nilpotency identity, Matematicheskii Sbornik 206, no. 11, 113-130 (2015). 
[26] A. V. Grishin, L. M. Tsybulya, A. A. Shokola, On T-spaces and relations in relatively free, Lie nilpotent, associative algebras, Journal of Mathematical Sciences 177 , no. 6 (2011).

[27] C. K. Gupta, A. Krasilnikov, Some non-finitely based varieties of groups and group representations, International Journal of Algebra and Computation 5, 343365 (1995).

[28] Narain Gupta, Frank Levin, On the Lie ideals of a ring, Journal of Algebra 81, 225-231 (1983).

[29] I. Kaplansky, Problems in the theory of rings, Report of a Conference on Linear Algebras, June, 1956, in National Acad. of Sci.-National Research Council, Washington, Publ. 502, 1-3 (1957).

[30] I. Kaplansky, Rings with a polynomial identity, Bulletin of the American Mathematical Society 54, 496-500 (1948).

[31] A. R. Kemer, Ideals of identities of associative algebras, Translations of Mathematical Monograph 97, American Mathematical Society, Providence (1988).

[32] D. Krakowski and A. Regev, The polynomial identities of the Grassmann algebra, Transactions of the American Mathematical Society 181, 429-438 (1973).

[33] A. Krasilnikov, The additive group of a Lie nipotent associative ring, Journal of Algebra 392, 10-22 (2013).

[34] V. N. Latyshev, On finite generation of a $T$-ideal with the element $\left[x_{1}, x_{2}, x_{3}, x_{4}\right]$, Sibirskii Matematicheskii Zhurnal (Siberian Mathematical Journal) 6, 1432-1434 (1965) (in Russian).

[35] V. N. Latyshev, On the choice of basis in a T-ideal, Sibirskii Matematicheskii Zhurnal (Siberian Mathematical Journal) 4, 1122-1126 (1963) (in Russian).

[36] $\mathrm{H}$. Laue, On the associated Lie ring the adjoint group of a radical ring, Canadian Mathematical Bulletin 27, no. 2 (1984).

[37] S. V. Okhitin, Central polynomials of an algebra of second-order matrices (Russian), Vestnik Moskovskogo Universiteta, Seriya I Matematika, Mekhanika 1988, no. 4, 61-63; English translation: Moscow University Mathematics Bulletin 43, 49-51 (1988).

[38] Yu. P. Razmyslov, Identities of Algebras and Their Representations, Translations of Mathematical Monographs, 138, American Mathematical Society, Providence, RI (1994). 
[39] V.V. Shchigolev, The finite basis property of $T$-spaces over fields of characteristic zero, Izvestiya Mathematics 65, 1041-1071 (2001).

[40] V.V. Shchigolev, Examples of infinitely based $T$-ideals, Fundamentalnaya i Prikladnaya Matematika 5 307-312 (1999).

[41] V.V. Shchigolev, Examples of T-spaces with an infinite basis, Matematicheskii Sbornik 191, 459-476 (2000).

[42] W. Specht, Gesetze in Ringen. I, Mathematische Zeitschrift 52, 557-589 (1950).

[43] A.N. Stoyanova-Venkova, The lattice of the variety of associative algebras defined by a commutator of length five, Comptes Rendus de L'Academie Bulgare des Sciences 34, 465-467 (1981).

[44] I. B. Volichenko. The T-ideal generated by the element $\left[x_{1}, x_{2}, x_{3}, x_{4}\right]$, Prepint no. 22, Institute of Mathematics of the Academy of Sciences of the Belorussian SSR, (1978) (in Russian).

[45] I. B. Volichenko, A. E. Zalesskii. Characterization of certain T-ideals from the view point of representation theory of the symmetric groups, Serdica Mathematical Journal 38, 211-236 (2012). 


\section{Índice Remissivo}

$T$-ideal, 21

finitamente gerado, 21

gerado por um conjunto, 21

multi-homogêneo, 15, 22

$T$-subálgebra, 25

T-subespaço, 25

finitamente gerado, 25

gerado por um conjunto, 25

multi-homogêneo, 15, 25

álgebra, 18

associativa, 18

livre, 19

associativa Lie nilpotente universal, 25

comutativa, 18

de Grassmann finitamente gerada e unitária, 20

de Grassmann infinitamente gerada e unitária, 20

de Lie, 24

de matrizes, 19

do grupo $G, 20$

relativamente livre, 25

unitária, 18

centro de uma álgebra, 25

componentes multi-homogêneas, 22

comutador, 19

endomorfismo, 21

grau

de um monômio, 21

de um polinômio, 21 homomorfismo, 21

ideal bilateral, 18

gerado por um conjunto, 18

identidade de Jacobi, 24

identidade polinomial, 19

lei anti-comutativa, 24

linearização total, 22

Pl-álgebra, 19

polinômio

m-central, 84

central, 25

hipercentral, 84

homogêneo, 22

multi-homogêneo, 22

multilinear, 22

próprio, 23

série central superior de uma álgebra, 84

subálgebra, 18

gerada por um conjunto, 18 